\title{
MD simulations of atomic hydrogen scattering from zero band-gap materials
}

\author{
Dissertation \\ zur Erlangung des mathematisch-naturwissenschaftlichen Doktorgrades \\ "Doctor rerum naturalium" \\ der Georg-August-Universität Göttingen \\ im Promotionsprogramm Chemie \\ der Georg-August University School of Science (GAUSS) \\ vorgelegt von \\ Marvin Kammler
}

aus Rühen

Göttingen, 2019 


\section{Betreuungsausschuss}

Prof. Alec M. Wodtke, Dynamik an Oberflächen, Georg August Universität, Göttingen

Prof. Ricardo Mata, Institut für Physikalische Chemie, Georg August Universität, Göttingen

Prof. Peter Saalfrank, Institut für Chemie, Universität Potsdam, Potsdam-Golm

\section{Mitglieder der Prüfungskommission}

Referent: Prof. Alec M. Wodtke, Dynamik an Oberflächen, Georg August Universität, Göttingen

Korreferent: Prof. Ricardo Mata, Computerchemie und Biochemie, Georg August Universität, Göttingen

Weitere Mitglieder der Prüfungskommission:

Prof. Peter Saalfrank, Institut für Chemie, Universität Potsdam, Potsdam-Golm

Prof. Claus Ropers, IV. Physikalisches Institut, Georg August Universität, Göttingen

Prof. Burkhard Geil, Biophysikalische Chemie, Georg August Universität, Göttingen

Prof. Jörg Behler, Theoretische Chemie, Georg August Universität, Göttingen

Tag der mündlichen Prüfung: 05.07.2019 



\section{Quotes}

A Change of Perspective is Worth 80 IQ Points. - Alan Kay

[T]HIS READINESS TO ASSUME THE GUILT FOR THE THREATS TO OUR ENVIRONMENT IS DECEPTIVELY REASSURING: We LIKE TO BE GUILTY SINCE, IF WE ARE GUILTY, IT ALL DEPENDS ON US. WE PULL THE STRINGS OF THE CATASTROPHE, SO WE CAN ALSO SAVE OURSELVES SIMPLY BY CHANGING OUR LIVES. WHAT IS REALLY HARD FOR US (AT LEAST IN THE WEST) TO ACCEPT IS THAT WE ARE REDUCED TO THE ROLE OF A PASSIVE OBSERVER WHO SITS AND WATCHES WHAT OUR FATE WILL BE. TO AVOID THIS IMPOTENCE, WE ENGAGE IN FRANTIC, OBSESSIVE ACTIVITIES. WE RECYCLE OLD PAPER, WE BUY ORGANIC FOOD, WE INSTALL LONG-LASTING LIGHT BULBS - WHATEVER - JUST SO WE CAN BE SURE THAT WE ARE DOING SOMETHING. WE MAKE OUR INDIVIDUAL CONTRIBUTION LIKE THE SOCCER FAN WHO SUPPORTS HIS TEAM IN FRONT OF A TV SCREEN AT HOME, SHOUTING AND JUMPING FROM HIS SEAT, IN THE BELIEF THAT THIS Will somehow influence the Game's outcome. - Slavoj ŽižEK

YOU CAN HAVE DATA WITHOUT INFORMATION, BUT YOU CANNOT HAVE INFORMATION WITHOUT DATA. - DANIEL KEYS MORAN

THE GOAL IS TO TURN DATA INTO INFORMATION, AND INFORMATION INTO INSight. - CARLY FIORINA

IF WE haVe DATA, LET'S LOOK AT DATA. IF ALl WE HAVE ARE OPINIONS, LET'S GO WITH MINE. - JIM BARKSDALE 


\section{Acknowledgments}

Foremost, I would like to express my sincere gratitude to Prof. Alec Wodtke for all the interesting and challenging projects I could be involved in. Your interest and support have advanced my understanding of the research topics to a great deal. I appreciate both the space you gave me and time you took to talk things over when necessary. Thank you very much.

My sincere thanks also goes to Dr. Sascha Kandratsenka for his supportive supervision. Your calmness and patience is enviable and I enjoyed every second talking to you inside or outside about work-related or random topics.

I also want to thank my thesis advisory committee which consists of Prof. Ricardo Mata and Prof. Peter Saalfrank, in addition to Prof. Wodtke. I feel very certain that you would have taken the time to discuss the issue had I met any insurmountable obstacles.

Next, I want to say thank you to all members of this group. It was always a great pleasure to have you around, whether is was making some music at one or the other event, playing kicker, or really any other time. I think you all are working conscientiously without being dogged which I also found very enjoyable.

Last, but not least, I want to thank my family for their love, support and encouragement they brought forth not only during my Ph.D., but during the entire time of my studies. I am also grateful to my friends in Göttingen and the greater Wolfsburg area who have been so welcoming time and time again during each of my visits. 


\begin{abstract}
This work deals with simulations of atomic hydrogen beam scattering from various surfaces. The studied surfaces are several (post-)transition metals, an insulator and graphene. Simulations were run with a self-written program that implements numerous Potential Energy Surfaces (PESs) to describe the different systems. All PESs used in this work were reparametrized with different approaches to accurately reproduce higher level reference data obtained from Density Functional Theory. These PESs were subsequently used to run classical Newtonian dynamics simulations of atomic beam scattering. Nuclear Quantum Effects (NQEs) that might arise in this process due to the small mass of the projectile can be accounted for by means of Ring Polymer Molecular Dynamics.

With the help of the simulations, one can gain valuable insight into scattering angle distributions, energy loss during the collision or sticking probabilities. These quantities can then be related to the incidence conditions and surface temperature. Isotope substitution can reveal the magnitude of NQEs and allows to estimate the kinetic isotope effect. The investigated surfaces exhibit a range of different properties. A metal surface with its unbound electrons causes the impinging particle to lose kinetic energy mostly due to electron-hole pair excitation. Different residence times at the surface lead to a very broad energy loss spectrum. The H-atom loses barely any energy when colliding with an insulator. Here, the energy transfer during the elastic scattering process is completely determined by the mass of the surface atoms.

When scattering from graphene, the particles can experience either a small or a large energy loss depending on the normal component of the incidence energy. In some situations, both energy loss channels are even apparent at the same time. This system is shown to rapidly accept much energy from a light collision partner without the need for nonadiabatic dynamics. Due to a simultaneous involvement of many degrees of freedom in the collision process, kinetic energy can quickly be transported away from the impact site. The characteristics of orbital (re-)hybridization paired with covalent bond formation opens up a novel energy dissipation pathway.
\end{abstract}




\section{Contents}

Acknowledgments

Abstract vi vi

1 Introduction 1

2 Theoretical Background 5

2.1 Born Oppenheimer Approximation . . . . . . . . . . . . 5

2.2 Density Functional Theory . . . . . . . . . . . . . . . . . . . . . 6

2.3 Potential Energy Surfaces . . . . . . . . . . . . . . . . . . . 10

2.3.1 Effective Medium Theory . . . . . . . . . . . . . . . 11

2.3.2 Reactive Empirical Bond Order . . . . . . . . . . . . . . . . 14

2.4 Molecular Dynamics . . . . . . . . . . . . . . . . . . . . . 17

2.4.1 Newtonian Dynamics . . . . . . . . . . . . . . . . . . . . . . . 19

2.4.2 Langevin Dynamics . . . . . . . . . . . . . . . . . . . . 20

2.4.3 Ring Polymer Molecular Dynamics . . . . . . . . . . . . . 21

2.4.4 Thermostats . . . . . . . . . . . . . . 24

2.5 Optimization Algorithms . . . . . . . . . . . . . . . 26

2.5 .1 Trust Region . . . . . . . . . . . . . . . . 27

2.5.2 Genetic Algorithm . . . . . . . . . . . . . . . 28

2.5.3 Fast Inertial Relaxation Engine . . . . . . . . . . . . . . 31

3 Methods 33

3.1 VASP . . . . . . . . . . . . . . . . . . 33

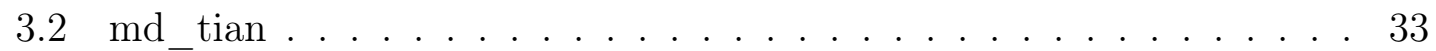

3.3 Skycruiser . . . . . . . . . . . . . . . . . . 35

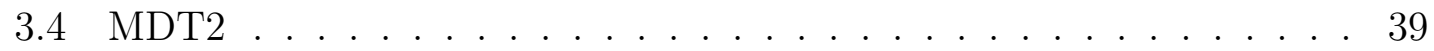

$4 \mathrm{H}$ on fcc Metals $\quad 45$

$4.1 \quad$ EMT PES . . . . . . . . . . . . . . . . . . . 46

4.1.1 Data Preparation . . . . . . . . . . . . . . 44 46

4.1 .2 Fit . . . . . . . . . . . . . . . . 55

4.1 .3 Discussion . . . . . . . . . . . . . . . 62

4.2 Scattering Results . . . . . . . . . . . . . . . . . . . . . 64

4.2.1 Adiabatic Simulation . . . . . . . . . . . . . . 65

4.2 .2 Nonadiabatic Simulation . . . . . . . . . . . . . . . 67 
4.2.3 Comparison with Experiment . . . . . . . . . . . . 77

4.3 PES Transferability . . . . . . . . . . . . . . . . . . . . . 81

4.3.1 (100) and (110) Surfaces . . . . . . . . . . . . 81

$4.3 .2 \quad \mathrm{Xenon}(111) \ldots \ldots \ldots \ldots$

4.4 Conclusion and Outlook . . . . . . . . . . . . . . . 88

$5 \mathrm{H}$ on Graphene $\quad 93$

$5.1 \quad$ REBO PES . . . . . . . . . . . . . . . . . . . . . . . 95

5.1 .1 PES Screening . . . . . . . . . . . . . . . 95

5.1 .2 Reparametrization .................. . 98

5.1.2.1 PBE Reference . . . . . . . . . . . . . . . . 99

5.1.2.2 EMFT Reference . . . . . . . . . . . . . . . 102

5.2 Detailed Dynamics . . . . . . . . . . . . . . . . . . . 108

5.2.1 Low Energy Collision . . . . . . . . . . . . . . . . . . . . 108

5.2 .2 Medium Energy Collision . . . . . . . . . . . . . . . . . 111

5.2.3 High Energy Collision . . . . . . . . . . . . . . . . . . . . 114

5.3 Sticking . . . . . . . . . . . . . . . . . 116

5.3.1 Comparison with EMFT . . . . . . . . . . . . 116

5.3 .2 Nuclear Quantum Effects . . . . . . . . . . . . . . . 117

5.3 .3 Substrate Effect . . . . . . . . . . . . . . . . . . . . 124

5.3.4 Comparison with Experiment . . . . . . . . . . . . 128

5.4 Energy Loss Distribution . . . . . . . . . . . . . . . . . . . . . 135

5.5 Conclusion and Outlook . . . . . . . . . . . . . . . . 139

References $\quad 143$

$\begin{array}{ll}\text { Acronyms } & 158\end{array}$ 


\section{Introduction}

Understanding gas-surface interactions is an important part of understanding the process of chemical change. It involves simultaneous motion of a shear infinite amount of atoms governed by the forces between them. Additionally, a surface is not a homogeneous, even and uniform atomic structure. There exist defects and steps between wider terraces whose ratio is given by the specific cut through the crystal. In consequence, some materials exhibit a structural reorganization of the top surface layers like the well-known herringbone reconstruction on the gold(111) surface. But also other topological deviations from the perfect model like the mentioned defects and accidental step formation may have a large influence on the reactivity of a surface. These imperfections are very difficult to get rid of. Hence it has proven to be a real challenge to characterize the effects of steps and terraces separately. One way to go about this situation and which has been made use of in the past, is to perform two experiments: one using a surface with a very low step density, and one where it is higher. Given the increased reactivity of surface atoms at steps due to missing neighbor atoms, a small fraction of them compared to all atoms at the surface may already have a notable influence on the reaction dynamics. The next step involves a formulation of a hypothesis which can then be put to the test using a surface cut with an intentionally higher step density.

Another challenge is that experiments are usually performed in thermal equilibrium. The measurement then consists of a convolution of the dynamics present on the surface. Untangling all the different processes which take place simultaneously but on different time scales is extremely difficult. The problem is that dynamic details are unknown. It would be simple the other way round, that is to say to derive the thermally averaged signal if the elementary reactions and corresponding rates were known. Numerous advances have been made during the last decades thanks to well-defined molecular beam experiments. A monoenergetic beam probing the reactivity only in a single direction, as opposed to a gas at thermal equilibrium in contact with a surface, is a great example of dimensionality reduction to simplify a problem. This approach paired with laser state-resolved studies of adsorption and scattering can already give meaningful insight into the different kinds of interaction at the atomic scale.

In case the important dynamics take place on the order of a few hundred femtoseconds, theory can provide valuable insights. Nevertheless, it is still difficult due to the large number of involved degrees of freedom (DOFs). If only a diatomic is 
taken into account, there are six of them which today can even be treated fully quantum mechanically. The larger problem is to find a suitable Potential Energy Surface (PES) which is accurate enough in all of these dimensions so that the use of wave packets is justified. As far as the interaction with a surface is concerned, six DOFs are by far not enough to capture energy transfer realistically. The coupling of surface DOFs to the center of mass motion of the impinging diatomic is most important. It determines whether enough kinetic energy can be transfered to the surface for it to adsorb and potentially dissociate. The reverse process is not any less interesting since it leads to desorption and diffusion. Vibrational coupling to phonons is usually weak because this motion happens too fast for any surface atoms to follow. It rather interacts with any available electronic DOFs. Rotational coupling is generally between these two extremes. Since translational energy transfer is crucial to describe any gas-surface interactions, this work is about H and D atoms interacting with metals surfaces and (quasi) free-standing graphene. This avoids to complicate the matter with any rotation or vibration, while still being of great importance towards understanding the fundamental forces that govern the interaction.

Nonadiabatic dynamics play a key role in the interaction between $\mathrm{H}$ atoms and metal surfaces. This implies that the Born-Oppenheimer Approximation (BOA) (section 2.1) breaks down to a certain amount which might limit the applicability of a single electronically adiabatic PES. It arises from the fact that there is a continuum of electronic states at a metal surface due to a missing band gap. An additional uncertainty is introduced by the velocity with which the projectile approaches the surface. In case it is too high for the electrons to immediately adapt to the changing nuclear coordinates, it might further restrict the validity of the BOA in this system. One way to deal with this situation is to use the Local Density Friction Approximation (LDFA) (section 2.4.2) to treat coupling to low-lying electronic states. In this approach, one uses a friction coefficient depending on the electron density that the impinging particle traverses leading to enhanced energy dissipation. It is a model that accounts for electron-hole pair (ehp) excitation which takes place due to a missing gap between valence and conduction band. The creation of hot electrons can be seen in experiment under certain conditions [1-3] which might also manifest itself in the form of chemicurrents [4,5]. Most of the time though, the BOA is a valid approximation. Theory for example can describe $\mathrm{H}$ atom scattering experiments from graphene with a single electronically adiabatic PES [6]. Graphene is also a zero band-gap material. But it seems that because valence and conduction band 
only touch at the $\Gamma$-point in the Brillouin zone, nonadiabatic dynamics do not play any role, at least not in the experimentally explored energy range.

The reason why scattering experiments can be difficult to interpret shall be briefly explained on the basis of NO scattering off of a $\mathrm{Ag}(111)$ surface. The impinging diatomic has only a weak attractive interaction with the surface. Experiments suggest that the N-down configuration is preferred with a well depth of $200 \mathrm{meV}$ [7]. At a high surface coverage and low temperature, two nitric oxide molecules decompose into an oxygen atom and a nitrous oxide molecule. A usual surface scattering experiment though happens in the limit of low coverage so that only the gas-surface interaction can be characterized. Any additional catalytic reactions only complicate the interpretation and are the subject of different sets of experiments. The advent of lasers allowed to study electronic, vibrational and rotational states of NO extremely precisely. Paired with time of flight techniques one can also gain knowledge about final kinetic energy distributions and rotatable detectors shed light on angular distributions.

Asada and Matsui noticed at the beginning of the 1980s that NO experiences a notable kinetic energy loss when scattering at high polar angles from an $\mathrm{Ag}(111)$ surface heated to $500 \mathrm{~K}[8,9]$. Other works report that rotational DOFs get excited as a consequence of this $[10,11]$ which was attributed to an anisotropic gas-surface interaction potential. They noticed the excitation of both low and high $J$-states, a definite non-Boltzmann distribution at the given temperature. This observation could later be ascribed to the rotational rainbow effect [10, 12]. In 1985, Rettner and coworkers observed that $5 \%$ of the NO approaching the $\mathrm{Ag}$ surface with $1 \mathrm{eV}$ incidence energy in $v=0$ ended up in the first excited vibrational state after direct scattering $[13,14]$. This was very unexpected for a system that is governed by a nonreactive PES [15] since it requires a head-on collision. Statistics and basic surface physics tell us that there are only very few of those and that phonons couple very inefficiently to the internal NO vibration. Hence, Rettner et al. favored an electronic mechanism for the vibrational excitation. Their suggestion was supported by the fact that the amount of detected NO in $v=1$ scales better with surface temperature than with incidence energy.

On the theory side, there were opposing views to describe the experimental observations. Tully et al. said that this mechanism is basically the reverse of ehp-mediated vibrational de-excitation [16] which was supported by Newns' nonadiabatic model [17]. A few years later however, two independent studies concluded that ehps are not essential for capturing the amount of vibrational excitation $[18,19]$ which started 
a discussion about the validity of the BOA for the description of this system. Experiments from the beginning of this century point again towards a nonadiabatic mechanism. The two main arguments are the observed vibrational de-excitation from NO starting in $v=2$ is more pronounced the higher the incidence energy [20], and the vibrational relaxation can involve several quanta when starting with NO in $v=15[21]$. As a side note, these last two experiments were performed on $\mathrm{Au}(111)$, but since multi-quanta de-excitation is not present when scattering from an insulator, the participation of ehps is today undisputed. The final evidence comes from a 2016 paper by Krüger and coworkers who measured a pronounced vibrational relaxation when scattering NO prepared in a high $v$-state from $\mathrm{Ag}(111)$ [22]. They made clear that a failure of the BOA is responsible for the observed results.

There are of course numerous other examples of surprising gas-surface interactions. Good summaries can for instance be found in Refs. [23, 24] which have greatly inspired this introduction and also myself to work on energy transfer dynamics at gas-surface interfaces. 


\section{Theoretical Background}

The purpose of this section is to explain the most important theoretical aspects that form the basis of this work. It is by far not exhaustive. Most of the topics can only be alluded to, but I try to provide references for further reading wherever possible. The information about the theoretical background, if not stated otherwise, is taken from Refs. [25-27] which provide a broad theoretical foundation and are great general reference works.

\subsection{Born Oppenheimer Approximation}

When we want to describe a system in its most general non-relativistic form, we make use of the time-dependent Schrödinger equation

$$
\hat{\mathcal{H}}(\boldsymbol{r}, \boldsymbol{R}, t) \Psi(\boldsymbol{r}, \boldsymbol{R}, t)=i \frac{\partial \Psi(\boldsymbol{r}, \boldsymbol{R}, t)}{\partial t} .
$$

The Hamilton operator $\hat{\mathcal{H}}$ is the sum of a position-dependent kinetic energy operator $\hat{\mathcal{T}}(\boldsymbol{r})$ and a position- and time-dependent potential energy operator $\hat{\mathcal{V}}(\boldsymbol{r}, t)$. Here, we denote the set of electron coordinates with $\boldsymbol{r}$ and the set of nuclear coordinates with $\boldsymbol{R}$. This is a linear partial differential equation, a transport equation and due to the imaginary unit $i$, also a wave equation. The highly nonlinear coupling terms which appear in multi-dimensional systems virtually prohibit solving it without any approximations.

First, we require that the potential energy operator be only a function of the set of atomic coordinates. This means that the Hamiltonian is time-independent and the total energy $E$ of the system is constant. Second, we assume that $\varphi(\boldsymbol{r}, t)$ can be written as a product of a position-dependent $\varphi(\boldsymbol{r})$ and a time-dependent function $f(t)$. Solving Eq. 2.1 with respect to time leads to

$$
\Psi(\boldsymbol{r}, \boldsymbol{R}, t)=\varphi(\boldsymbol{r}, \boldsymbol{R}) f(t)=\varphi(\boldsymbol{r}, \boldsymbol{R}) \mathrm{e}^{-i E t}
$$

Since the Hamiltonian is a hermitian operator, its eigenvalues are real and the timedependence is purely oscillatory and does not vary in magnitude. Hence, this factor can be ignored for time-independent problems.

$\hat{\mathcal{H}}$ now only depends on the spatial coordinates of the system and can be expanded 
as

$$
\hat{\mathcal{H}}(\boldsymbol{r}, \boldsymbol{R})=\hat{\mathcal{T}}_{\mathrm{n}}(\boldsymbol{R})+\hat{\mathcal{T}}_{\mathrm{e}}(\boldsymbol{r})+\hat{\mathcal{V}}_{\mathrm{nn}}(\boldsymbol{R})+\hat{\mathcal{V}}_{\mathrm{ne}}(\boldsymbol{r}, \boldsymbol{R})+\hat{\mathcal{V}}_{\mathrm{ee}}(\boldsymbol{r})
$$

where the subscript $n$ describes the nuclei and subscript $e$ indicates electrons. Due to their opposite charge, they both feel the same mutually attractive force. Nevertheless, their respective masses are separated by multiple orders of magnitude which leads us to the conclusion that the electrons must be moving much faster than the nuclei. We use this knowledge to decouple the nuclear and electronic motion in the expression of $\hat{\mathcal{H}}$ to create an electronic Hamiltonian $\hat{\mathcal{H}}_{\mathrm{e}}$

$$
\begin{aligned}
{\left[\hat{\mathcal{T}}_{\mathrm{e}}(\boldsymbol{r})+\hat{\mathcal{V}}_{\mathrm{nn}}(\boldsymbol{R})+\hat{\mathcal{V}}_{\mathrm{ne}}(\boldsymbol{r}, \boldsymbol{R})+\hat{\mathcal{V}}_{\mathrm{ee}}(\boldsymbol{r})\right] \Psi(\boldsymbol{r} ; \boldsymbol{R}) } & =\hat{\mathcal{H}}_{\mathrm{e}} \Psi(\boldsymbol{r} ; \boldsymbol{R}) \\
& =E_{\mathrm{e}}(\boldsymbol{R}) \Psi(\boldsymbol{r} ; \boldsymbol{R})
\end{aligned}
$$

which yields electronic energies $E_{\mathrm{e}}$. The semicolon in Eq. 2.4 denotes that the wave function now only depends parametrically on the nuclear coordinates. It still takes into account the nuclear positions but neglects their velocities. The total energy of the system is then obtained in a second step via

$$
\left[\hat{\mathcal{T}}_{\mathrm{n}}(\boldsymbol{R})+E_{\mathrm{e}}(\boldsymbol{R})\right] \Psi_{\mathrm{n}}(\boldsymbol{R})=E_{\mathrm{tot}} \Psi_{\mathrm{n}}(\boldsymbol{R})
$$

As long as the electronic ground state is well separated in terms of energy from any excited state, the BOA (Eq. 2.4) keeps its validity. Even for the lightest molecule $\mathrm{H}_{2}$, the error is only a few meV which is much less than the expected Density Functional Theory (DFT) error (section 2.2) and gets even smaller for heavier nuclei. The $\mathrm{BOA}$ is also the justification for the use of PESs (section 2.3) to describe interaction on an atomic scale. They are the foundation for fast Molecular Dynamics (MD) simulations (section 2.4) on which a major part of this work is based. [28, 29]

\subsection{Density Functional Theory}

DFT is a widely used approach to calculate properties of molecular systems like energies, geometries and electronic densities among many others. A detailed description of DFT can be found in any computational chemistry textbook. Here I will only introduce the most general concepts and highlight aspects which are important for this work.

The foundation of DFT is the Hohenberg-Kohn existence theorems [30]. The first 
theorem states that there is a unique mapping from the ground state electronic density $\rho_{0}$ onto the ground state energy $E_{0}$. This finding allows one to formulate a Schrödinger equation using the electronic density instead of a wave function. The second theorem argues that the energy is variational with respect to the density. In consequence, any trial density distribution that does not coincide with the true ground state density necessarily leads to a higher energy. E. B. Wilson remarked that even without any formulas, one can see already from intuition that the density should be a useful measure. First, integration over the entire space yields the number of electrons in the system, second, the position of the nuclei are defined by cusps in the density and third, their height corresponds to the nuclear charge. [31]

Another advantage of DFT is that the theory is in principle orbital-free. The density is merely a function of three Cartesian coordinates, whereas a wave function (Eq. 2.1) would require three coordinates for each electron. Unfortunately, one cannot yet profit from this great reduction in dimensionality for reasons that shall be described in the next paragraph.

Just as in the Schrödinger equation, we can split the Hamiltonian in several operators. The kinetic energy operator $\hat{\mathcal{T}}[\rho]$, nuclear-electron $\hat{\mathcal{V}}_{\text {ne }}[\rho]$ and the electronelectron $\hat{\mathcal{V}}_{\text {ee }}[\rho]$ potential energy operators. The latter can be further fragmented in Coulomb $\hat{\mathcal{J}}[\rho]$ and exchange $\hat{\mathcal{K}}[\rho]$ parts. The nuclear-nuclear interaction is a constant within the BOA. Two of the operators can be expressed exactly by

$$
\begin{gathered}
\hat{\mathcal{V}}_{\text {ne }}[\rho]=-\sum_{i}^{N_{\text {nuclei }}} \int \frac{Z_{i}\left(\boldsymbol{R}_{i}\right) \rho(\boldsymbol{r})}{\left|\boldsymbol{R}_{i}-\boldsymbol{r}\right|} \mathrm{d} \boldsymbol{r} \\
\hat{\mathcal{J}}[\rho]=\frac{1}{2} \iint \frac{\left|\rho(\boldsymbol{r}) \rho\left(\boldsymbol{r}^{\prime}\right)\right|}{\boldsymbol{r}-\boldsymbol{r}^{\prime}} \mathrm{d} \boldsymbol{r} \mathrm{d} \boldsymbol{r}^{\prime}
\end{gathered}
$$

but $\hat{\mathcal{T}}[\rho]$ and $\hat{\mathcal{K}}[\rho]$ can only be expressed exactly in case of the uniform electron gas. While this model system might hold for metallic systems, it definitely performs poorly on isolated molecules.

One way to approximate $\hat{\mathcal{T}}[\rho]$ for nonuniform density distributions is to use a Taylor expansion. [32, 33]

$$
\begin{aligned}
& \hat{\mathcal{T}}[\rho]=\hat{\mathcal{T}}_{0}[\rho]+\hat{\mathcal{T}}_{2}[\rho]+\hat{\mathcal{T}}_{4}[\rho]+\hat{\mathcal{T}}_{6}[\rho]+\ldots \\
& \hat{\mathcal{T}}_{0}[\rho]=\frac{3}{10}\left(3 \pi^{2}\right)^{\frac{2}{3}} \int \rho^{\frac{5}{3}}(\boldsymbol{r}) \mathrm{d} \boldsymbol{r} \\
& \hat{\mathcal{T}}_{2}[\rho]=\frac{1}{72} \int \frac{|\nabla \rho(\boldsymbol{r})|^{2}}{\rho(\boldsymbol{r})} \mathrm{d} \boldsymbol{r}
\end{aligned}
$$




$$
\begin{aligned}
\hat{\mathcal{T}}_{4}[\rho]=\frac{\left(3 \pi^{2}\right)^{-\frac{2}{3}}}{540} \int & \rho^{\frac{1}{3}}(\boldsymbol{r})\left[\left(\frac{\nabla^{2} \rho(\boldsymbol{r})}{\rho(\boldsymbol{r})}\right)^{2}\right. \\
& \left.-\frac{9}{8}\left(\frac{\nabla^{2} \rho(\boldsymbol{r})}{\rho(\boldsymbol{r})}\right)\left(\frac{\nabla \rho(\boldsymbol{r})}{\rho(\boldsymbol{r})}\right)^{2}+\frac{1}{3}\left(\frac{\nabla \rho(\boldsymbol{r})}{\rho(\boldsymbol{r})}\right)^{4}\right] \mathrm{d} \boldsymbol{r}
\end{aligned}
$$

and the same approach can also be used to describe $\hat{\mathcal{K}}[\rho]$. [34, 35]. These expansions only contain even elements due to rotational invariance. Typically, $\hat{\mathcal{T}}_{0}[\rho]$ underestimates the kinetic energy by about $10 \%$ and inclusion of $\hat{\mathcal{T}}_{2}[\rho]$ reduces the error to $1 \%$. However, adding more terms does not improve the accuracy, but quite the contrary. $\hat{\mathcal{T}}_{6}[\rho]$ and higher terms, besides being incredibly lengthy and difficult to derive, diverge at the center of the nuclei and far away from them. Some attempts have been made to stop the Taylor sequence after the second term and introduce corrections by either changing the prefactors, or using linear response theory with second-order reduced density matrices. Nevertheless, the accuracy and transferability of orbital-free methods still lags behind modern standards and are seldom used. [36]

Interestingly, the reintroduction of orbitals for the use in DFT circumvents the problem of the badly represented kinetic energy expression. Kohn and Sham (KS) used the approach of calculating as many terms as possible exactly using orbitals and moving all unknown exchange-correlation contributions into a functional-dependent term $E_{\mathrm{xc}}$. This makes KS-DFT very similar to Hartree-Fock (HF) in that electrons are treated as noninteracting particles with a kinetic energy of

$$
T_{\mathrm{KS}}=\sum_{i=1}^{N_{\text {elec }}}\left\langle\varphi_{i}\left|-\frac{1}{2} \nabla^{2}\right| \varphi_{i}\right\rangle .
$$

Neglecting the electron kinetic correlation energy leads to an error of about $1 \%$, which is a much better value than any orbital-free method can provide on a regular basis. In absolute numbers, the kinetic energy can be expected to be one order of magnitude larger than the exchange-correlation energy. In consequence, any errors in the former term carry much more weight.

In addition to the correction of the kinetic energy of the electrons, $E_{\mathrm{xc}}$ also contains three more terms which cannot be expressed exactly in the KS picture. Electrons adjust their movement due to the electric field generated by other electrons, which reduces the Coulomb repulsion. For a one-electron system, this term also cancels the self-interaction error of a density with itself (cf. Eq. 2.7). Moreover, $E_{\mathrm{xc}}$ takes 
care of quantum mechanical exchange which further lowers Coulomb repulsion of electrons with the same spin.

These are a lot of requirements on a single expression. If only the density is used as an argument, this approach is called Local Density Approximation (LDA) and it yields acceptable energies for systems of slowly varying density like bulk metals. As refinement step one can use a Taylor-expansion of the density to improve the exchange-correlation energy, just like in Eq.2.8. Functionals that make additional use of the gradient in the evaluation of $E_{\mathrm{xc}}$ belong to the class of the Generalized Gradient Approximation (GGA). They usually outperform LDA functionals in terms of energy barriers, atomization energies and equilibrium structures when compared with high-level wave functional methods [37, 38]. The PBE functional [39] was used extensively in this work for the description of metal-hydrogen interaction and belongs to this class.

One way to go beyond the GGA is to include exact HF exchange [40]. These so called Hybrid Functionals are about 50 times slower than GGAs [41] but may reward the user with great accuracy when treating larger systems for which sophisticated wave function-based methods are infeasible. The only Hybrid Functional employed in this work is B3LYP $[42,43]$ which was used in an embedded approach called Embedded Mean-Field Theory (EMFT) [44] to describe hydrogen on graphene. More details about the calculation of $E_{\mathrm{xc}}$ using the electron density and derivatives thereof can be found in Ref. [45].

Due to the described approximations, there are several aspects of DFT which are known to be problematic. First and foremost, the locality of all the methods may be a cause of errors. Though GGAs and meta-GGAs incorporate information about first and second derivatives of the density, adding more terms of the Taylor expansion does not solve the problem of locality. Any long range effects are not captured by DFT. However, van der Waals interactions can be artificially included as an additive term in the energy expression by using, for example, the Grimme [46] or Tkatchenko-Scheffler [47] method. Second, the exchange-correlation functional exhibits a flawed asymptotic behavior at large distances, i.e., they decay as $\exp (-r)$ instead of $-r^{-1}$. Consequently, negatively charged systems with a low electron affinity and Rydberg states are not stable because the self-interaction term exceeds the binding energy. However, there exist Asymptotically Corrected functionals which are specifically designed for calculating vertical binding energies, charge transfer states or static polarizabilities $[48,49]$. Third, exited states are difficult to model because the Hohenberg-Kohn theorems restrict DFT to only work for (non- 
degenerate) ground states. Especially if both states are equal in spin and symmetry, it is impossible to enforce orthogonality using the electron density. Time-dependent DFT [50] may be used to obtain excited state properties, but results always have to be checked very carefully [51]. Fourth, DFT cannot be systematically improved. There exists no hierarchy of methods that approaches the exact result. However, different functionals have demonstrated empirically to give similar results and that their performance is rather insensitive to their constituent details [52].

The main motivation for choosing DFT is the good ratio of computational effort to performance. In the case of scattering processes, the simulation cell needs to meet certain minimum requirements. For example, the impinging projectile must feel the interaction with an infinite surface. Using only a handful of atoms in the shape of a cluster will not be a good representation of about $10^{15}$ surface atoms per square centimeter in the experiment. Periodic boundary conditions are therefore essential to model an extended system while keeping the actual number of simulated atoms manageable. Nevertheless, the simulation cell needs to contain enough atoms so that the projectile neither suffers from image-phonon recurrence effects nor from self-interaction due to periodic boundary conditions. Self-interaction in this sense refers to the interaction of a particle across repeated images of the cell in the $x y$-plane andor coupled cluster [53] are impractical for the description of extended systems due to their huge computational cost.

\subsection{Potential Energy Surfaces}

PESs are continuous mathematical functions that map the atomic positions of a system onto its potential energy. Their formulation is a direct consequence of the BOA, because their use implies that all electrons in the system respond instantaneously to a new nuclear configuration. One can think of a PES as a potential energy landscape in which atoms are moving. Following Newton's equations of motion, nuclear kinetic energy can be converted into potential energy and vice versa. Usually, PESs convert the given atomic positions into internal coordinates, as the system is equally well described by interatomic distances, bond angles and dihedral angles. This procedure ensures the translational and rotational invariance with respect to the energy and keeps the number of degrees of freedom unchanged. Since the PES is a smooth and continuous function, forces on the atoms can be quickly calculated via analytic derivatives. Fig. 1 shows the Lennard-Jones (LJ) potential [54] of two Xenon atoms as an example of a simple potential energy curve, i.e., a PES 


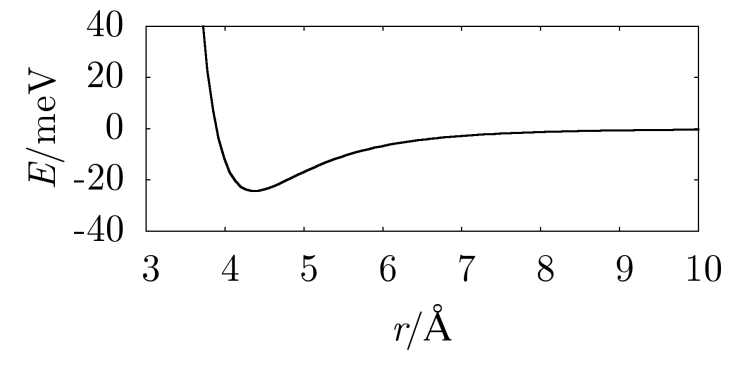

(a)

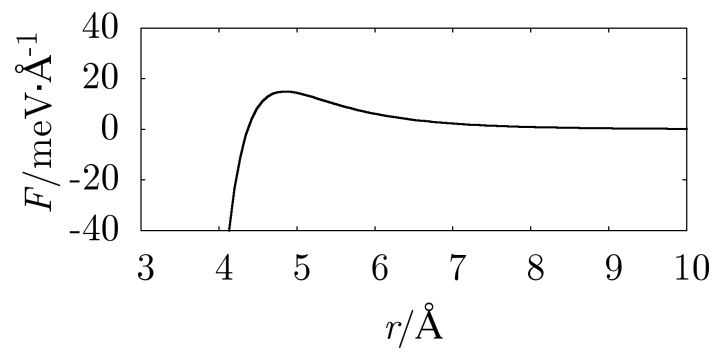

(b)

Figure 1: LJ potential energy (a) and force (b) describing the interaction of two Xenon atoms. $\varepsilon=24.326 \mathrm{meV}, \sigma=3.8924 \AA$, parameters taken from Ref. [55].

in two dimensions. This potential was used in this work to describe any long-range, induced-dipole interaction between atoms. Its energy $E$ and force $F$ are given by

$$
\begin{gathered}
E(r)=4 \varepsilon\left[\left(\frac{\sigma}{r}\right)^{12}-\left(\frac{\sigma}{r}\right)^{6}\right] \\
-\frac{\mathrm{d} E}{\mathrm{~d} r}=F(r)=24 \varepsilon\left[\left(\frac{\sigma}{r}\right)^{6}-2\left(\frac{\sigma}{r}\right)^{12}\right] r^{-1} .
\end{gathered}
$$

Since this function only takes bond distances into account, it has to be evaluated $0.5 N(N-1)$ times for a system of $N$ Xe-atoms. Due to its mathematical simplicity and a scaling of $\mathcal{O}\left(n^{2}\right)$, tens of thousands of atoms can routinely be studied in a simulation cell. More sophisticated potentials used in this work are Effective Medium Theory also exhibiting a potential energy scaling of $\mathcal{O}\left(n^{2}\right)$ and the second generation Reactive Empirical Bond Order which scales with $\mathcal{O}\left(n^{4}\right)$.

\subsubsection{Effective Medium Theory}

Effective Medium Theory (EMT) was invented by Jacobsen et al. to model impurities in metals $[56,57]$. Their ideas can only be outlined in this section and the presented formulas are only valid for a single atomic species. More detailed information can be obtained from the original publications. Since metals crystallize in a periodic structure and the electronic density varies only slowly, the Uniform Electron Gas (UEG) is a fair approximation as a reference system. Furthermore, a lot of properties of the UEG can be derived analytically. In consequence, it is easier to model only differences between the UEG and any real system than to model the real system without a physically motivated support structure. This understanding is the key concept of EMT and has proven to provide good estimates to heats of solution, vacancy formation energies and alloy structures of metallic systems [57]. Interest- 
ingly, this PES can also describe an $\mathrm{H}$ atom as an impurity on a metal surface and in the bulk, which makes it appealing for use in $\mathrm{H}$ atom scattering simulations [58].

EMT describes all atomic species as having isotropic, non-directional bonds. The only geometry-defining quantity is the radius. For a face-centered cubic (fcc) structure, this is a reasonable approximation because the first Brillouin zone has the shape of the truncated octahedron which has a sphericity of 0.91 [59]. Since solids as a whole do not carry any charge, the same must be true for the unit cell. The unit cell in an fcc metal only consists of one atom, hence the truncated octahedron must be charge-free. The neutral sphere radius parameter $s_{0}$ in EMT now defines a sphere with the same volume as the truncated octahedron. This is of course a simplified description of a metal, but reasonable to model isotropic bonding.

The starting point for the total energy of the metallic system is the sum over each atom's cohesive energy, i.e., the sublimation energy of one atom. This cohesive energy $E_{\mathrm{c}}$ differs among the atoms because distances to their nearest neighbors fluctuate due to temperature. The deviation of $E_{\mathrm{c}}$ as a function of the neutral sphere radius $s$ is given by

$$
E_{\mathrm{c}}(s)=E_{0}\left[1+\lambda\left(s-s_{0}\right)\right] e^{\lambda\left(s-s_{0}\right)}
$$

with $E_{0}$ as the equilibrium cohesive energy, $s_{0}$ as the equilibrium neutral sphere radius and $\lambda$ as a sensitivity parameter. Continuing the discussion of the reference system, there will be differences of course between the UEG and our system of interest. Jacobsen and coworkers combined the differences in electrostatic and exchange-correlation energy into a term called the atomic sphere correction $\Delta E_{\mathrm{AS}}$. Together with $E_{\mathrm{c}}$, we can now formulate the total energy expression of EMT

$$
\begin{aligned}
E & =\sum_{i}\left[E_{\mathrm{c}, i}\left(n_{i}\right)+\Delta E_{\mathrm{AS}}(i)\right] \\
& =\sum_{i}\left\{E_{\mathrm{c}, i}\left(n_{i}\right)+\frac{1}{2}\left[\sum_{j \neq i} V_{i j}\left(r_{i j}\right)-\sum_{j \neq i}^{\mathrm{ref}} V_{i j}\left(r_{i j}\right)\right]\right\} .
\end{aligned}
$$

$n_{i}$ is the electron density around atom $i$ and $V_{i j}\left(r_{i j}\right)$ is a pair-potential which explains the scaling of $\mathcal{O}\left(n^{2}\right)$ with respect to the number of atoms. The equation also shows the ingenious use of the pair-potential approximation in EMT. Instead of directly using a pair-potential, Jacobsen et al. chose to model the correction term $\Delta E_{\mathrm{AS}}$ via a difference of two pair-potentials. One describes the real system and the other the 
reference system. Its functional form is

$$
V(r)=-V_{0} \exp \left[-\frac{\kappa}{\beta}\left(r_{i j}-\beta s_{0}\right)\right]
$$

with $V_{0}$ and $\kappa$ being further EMT parameters and $\beta$ as a geometry-dependent conversion factor relating nearest neighbor distance to neutral sphere radius.

The neutral sphere radius of atom $i$ is calculated as

$$
\begin{aligned}
\sigma_{i} & =\sum_{j \neq i} e^{-\eta_{2}\left(r_{i j}-\beta s_{0}\right)}, \\
s_{i} & =s_{0}-\frac{1}{\beta \eta_{2}} \log \left(\frac{\sigma_{i}}{12}\right) .
\end{aligned}
$$

with $\eta_{2}$ as a density fall-off parameter. The exponential decay of the electron density is based on DFT from which it natively emerges in the case of the UEG. Paired with the variational principle of the total energy functional, errors in the electron density only lead to second and higher order errors in the total energy. Dividing by 12 accounts for the fact that each atom has 12 nearest neighbors in the fcc reference system.

One useful quantity that can be derived from EMT is the electron density which is defined by a parameter $n_{0}$ and the aforementioned exponential decay

$$
n_{i}=\sum_{j \neq i} \frac{n_{0}}{12} \exp \left[\eta_{1}\left(s_{i}-s_{0}\right)-\eta_{2}\left(r_{i j}-\beta s_{0}\right)\right]
$$

This density will be essential for modeling nonadiabatic effects in terms of electronhole pair excitation (cf. section 2.4.2).

The EMT parameters can furthermore be related to macroscopic measures like the bulk modulus $B$ and the three elastic constants $C_{\mathrm{xx}}$ of an fcc lattice. They are given by

$$
\begin{aligned}
C_{11} & =\frac{3 V_{0}\left(\beta \eta_{2}-\kappa\right) \kappa-E_{0} \lambda^{2}}{12 \pi s_{0}} \\
C_{12} & =\frac{3 V_{0}\left(\kappa-\beta \eta_{2}\right) \kappa-2 E_{0} \lambda^{2}}{24 \pi s_{0}} \\
C_{44} & =\frac{3 V_{0}\left(\beta \eta_{2}-\kappa\right) \kappa}{8 \pi s_{0}}=C_{11}-C_{12} \\
B & =-V \frac{\mathrm{d}^{2} E}{\mathrm{~d} V^{2}}=-\frac{E_{0} \lambda^{2}}{12 \pi s_{0}}=\frac{1}{3}\left(C_{11}+2 C_{12}\right) .
\end{aligned}
$$


Their flexibility is limited, though, since only two of them are linearly independent. In consequence, all materials described by EMT have a fixed shear anisotropy ratio (Zener anisotropy) [60] of

$$
A=\frac{2 C_{44}}{C_{11}-C_{12}}=2
$$

In reality, the Zener anisotropy varies between one and three for the metals considered in this work and fortunately is not a crucial quantity to accurately describe scattering processes. The bulk modulus is related to the melting temperature of the metal and can be adjusted by fitting the $\lambda$ parameter since $E_{0}$ and $s_{0}$ are easily accessible. $B$ and $E_{0}$ are tabulated in the literature and $s_{0}$ can be calculated from the lattice constant obtained from DFT. Another viable option would be to use DFT to get information about $B$ by fitting the Murnaghan equation of state [61].

\subsubsection{Reactive Empirical Bond Order}

The first paper about the Reactive Empirical Bond Order (REBO) potential was published by Brenner in 1990 [62] and dealt with chemical vapor deposition of diamond. The goal was to elucidate the mechanism by which diamonds can be grown under metastable conditions, to identify the species that adds to the surface, to locate the binding site (surface terrace or step) and many more details about the process. He followed the approach of Abell [63] who developed an analytical expression to describe a general binding energy curve [64] as a function of the local atomic environment. Tersoff extended Abell's ideas by designing a function to model the potential energy of many solid-state structures [65]. Starting with silicon, his ideas were readily adopted to perform calculations on main group IV-IV [66] and III-V [67] compounds. These all-purpose potentials are known today as the Abell-Tersoff type and have spawned an entire generation of PESs.

Brenner defined four criteria on which a PES should be rated [68]. He used them as a foundation on which he built his famous second generation REBO potential. First, the potential must be flexible. It must be able on the one hand to model a large variety of structures from the fitting database and on the other hand derive a number of properties like cohesive and vacancy formation energies for solid state structures, for example. Second, the accuracy is of crucial importance. The fitting error of binding energies and bond lengths in the training dataset must be as small as possible. Third, the potential function must be transferable. There should be at least a qualitative description of structures not included in the training data. For 
instance, it should be able to determine the energetically most favorable adsorption site, though the adsorption energy may not be quantitative. Last, the development of a PES must happen with computational efficiency in mind. There would simply be no advantage over DFT if the function evaluation took the same amount of time.

Using the Abell-Tersoff potential as a starting point and with all these aspects of a well-designed PES in mind, Brenner developed the second generation REBO potential [69]. This potential was used in this work to describe the interaction of a hydrogen atom with a graphene sheet and will be briefly described in this section. The total energy $E_{\mathrm{b}}$ is given by

$$
E_{\mathrm{b}}=\frac{1}{2} \sum_{i} \sum_{j \neq i}\left[V^{\mathrm{R}}\left(r_{i j}\right)-b_{i j} V^{\mathrm{A}}\left(r_{i j}\right)\right]
$$

and consists of a repulsive $V^{\mathrm{R}}$ and an attractive term $V^{\mathrm{A}}$. In line with the arguments of Rose et al. [64], both terms exhibit an exponential dependence on $r$. Within the REBO formalism, they are

$$
\begin{aligned}
& V^{\mathrm{R}}\left(r_{i j}\right)=f^{\mathrm{c}}\left(r_{i j}\right)\left(1+\frac{Q}{r_{i j}}\right) A \mathrm{e}^{-\alpha r_{i j}} \\
& V^{\mathrm{A}}\left(r_{i j}\right)=f^{\mathrm{c}}\left(r_{i j}\right) \sum_{n=1}^{3} B_{n} \mathrm{e}^{-\beta_{n} r_{i j}}
\end{aligned}
$$

in which the function

$$
f^{\mathrm{c}}\left(r_{i j}\right)= \begin{cases}1 & r_{i j} \leq d_{\min } \\ \frac{1}{2}\left[1+\cos \left(\pi \cdot \frac{r_{i j}-d_{\min }}{d_{\max }-d_{\min }}\right)\right] & d_{\min }<r_{i j}<d_{\max } \\ 0 & r_{i j} \geq d_{\max }\end{cases}
$$

ensures a continuous and differentiable cutoff over the distance $d_{\max }-d_{\min }$. All other parameters serve stretching and shifting requirements of the functions.

The only variable left to explain is the empirical bond order $b_{i j}$ in Eq. 2.24. Calling it 'empirical' is important to distinguish it from the traditional notion of a bond order in terms of single, double and triple bonds having a bond order of one, two and three, respectively. It is rather dependent on the close environment and scales as the inverse square root of the local coordination as can be derived from the second moment approximation to the electron density distribution [70]. This methodology is also applied in Tight Binding theory [71] which is a simplification of the Harris 
energy functional [72] which, in turn, is an offshoot of DFT. Hence, the underlying basis of REBO is built upon a very strong argument. The difficult part, however, is to find equations that take the local coordination into account in a reasonable way. Therefore, $b_{i j}$ contains contributions from sigma-pi interactions $b^{\sigma-\pi}$, a term to describe any radical character $\Pi^{\mathrm{RC}}$ and one for dihedral angles $b^{\mathrm{DH}}$ resulting in

$$
b_{i j}=\frac{1}{2}\left(b_{i j}^{\sigma-\pi}+b_{j i}^{\sigma-\pi}\right)+\Pi_{i j}^{\mathrm{RC}}+b_{i j}^{\mathrm{DH}} .
$$

$b^{\sigma-\pi}$ realizes the inverse square root scaling and depends on the bond angle between atoms $i, j$ and $k$. It is given by

$$
b_{i j}^{\sigma-\pi}=\left\{1+\sum_{k(\neq i, j)} f_{i k}^{\mathrm{c}}\left(r_{i k}\right) G\left[\cos \left(\theta_{i j k}\right)\right] \mathrm{e}^{\lambda_{i j k}}+P_{i j}\left(N_{i}^{\mathrm{C}}, N_{i}^{\mathrm{H}}\right)\right\}^{-\frac{1}{2}}
$$

where $G$ and $P_{i j}$ are $1 \mathrm{D}$ and $2 \mathrm{D}$ splines, respectively, and $N_{i}^{\mathrm{C}}$ and $N_{i}^{\mathrm{H}}$ are the number of neighboring carbon and hydrogen atoms of atom $i . \lambda_{i j k}$ is a parameter that depends on the atomic species of atoms $i, j$ and $k$.

The radical character of a bond is determined by a tricubic spline that depends on the total number of neighbors $N^{\mathrm{t}}=N^{\mathrm{C}}+N^{\mathrm{H}}$ of atoms $i$ and $j$ and the conjugation of the bond between them. It is required to describe non-local conjugation as present in benzene and graphitic structures and to model vacancy formation. The conjugation $N_{i j}$ is given by

$$
\begin{aligned}
N_{i j}^{\mathrm{conj}} & =1+\left[\sum_{k(\neq i, j)}^{\text {carbon }} f_{i k}^{\mathrm{c}}\left(r_{i k}\right) F\left(x_{i k}\right)\right]^{2}+\left[\sum_{l(\neq i, j)}^{\text {carbon }} f_{j l}^{\mathrm{c}}\left(r_{j l}\right) F\left(x_{j l}\right)\right]^{2} \\
F\left(x_{i k}\right) & = \begin{cases}1 & x_{i k} \leq 2 \\
\frac{1}{2}\left\{1+\cos \left[2 \pi\left(x_{i k}-2\right)\right]\right\} & 2<x_{i k}<3 \\
0 & x_{i k} \geq 3\end{cases} \\
x_{i k} & =N_{k}^{\mathrm{C}}+N_{k}^{\mathrm{H}}-f_{i k}^{\mathrm{c}}\left(r_{i k}\right) .
\end{aligned}
$$

This is a highly flexible description of conjugation and is part of the improvement over the first generation REBO PES [62]. For a completely saturated material like diamond $N_{i j}^{\text {conj }}$ equals one, it equals three for benzene, and it is nine in graphitic systems. Conjugation effects also contribute to the dihedral term in the bond order 
which is modeled by

$$
b_{i j}^{\mathrm{DH}}=T_{i j}\left(N_{i}^{\mathrm{t}}, N_{j}^{\mathrm{t}}, N_{i j}^{\mathrm{conj}}\right)\left\{\sum_{k(\neq i, j)} \sum_{l(\neq i, j, k)}\left[1-\cos ^{2}\left(\Theta_{k i j l}\right)\right] f_{i k}^{\mathrm{c}}\left(r_{i k}\right) f_{j l}^{\mathrm{c}}\left(r_{j l}\right)\right\}
$$

where $\Theta_{k i j l}$ is the angle between the normal vectors of the planes defined by atoms $k-i-j$ and $i-j-l$. The double sum is zero for parallel normal vectors and it equals one if they are orthogonal to each other. This is a standard approach to model the barrier to rotation about conjugated carbon bonds. The height of the barrier is determined by $T_{i j}$ which is, again, a tricubic spline function allowing for interpolation between non-integer values of $N^{\mathrm{t}}$ and $N_{i j}^{\text {conj }}$. The nodes of all spline functions along with parameters for $\mathrm{C}$ and $\mathrm{H}$ atoms in Eqns. (2.25)-(2.27) can be found in the original publication [69]. The splines were fitted to a mixture of theoretically and experimentally obtained force constants, binding energies, bond lengths, elastic properties and other characteristics of small organic molecules and extended graphitic structures. Altogether, the revised functional forms paired with the large fitting database outperforms the first generation REBO potential as well as the Tersoff potential when compared with high-quality density functional calculations.

\subsection{Molecular Dynamics}

With a reasonable PES at hand, one can easily determine equilibrium geometries, cohesive energies and all other kinds of static properties. We start by looking at the ergodic hypothesis which states that an arbitrary system average $\langle X\rangle$ can be either obtained by monitoring one particle for an infinite amount of time or by looking at infinitely many particles at one moment in time. If we take the ensembleaverage route, we arrive at Monte Carlo techniques that generate points in phase space by random displacements of atoms. But this is not the way to successfully modeling $\mathrm{H}$ atom scattering since this is clearly a momentum- and time-dependent phenomenon. MD on the other hand generates correlated points in phase space which means time-averaging will yield $\langle X\rangle$. Starting from an initial configuration of the system, time-correlation in phase space is enforced by Newton's equation of motion (section 2.4.1). The entire sequence of points is called a trajectory and shows how the system evolves over time.

These are several prerequisites for running an MD simulation. First, a mapping from atomic coordinates to an energy, i.e., via a potential energy function, is nec- 
essary but not sufficient. Forces are required as well which, depending on the PES, can range from simple to very complex analytical expressions. Numerical forces that require only the knowledge of the energy function can in principle be used instead, but they require much more computation time than analytical forces. Second, a time step has to be determined which on the one hand should be as large as possible to maximize simulated time and on the other hand be small enough to ensure energy conservation. A time step of $0.1 \mathrm{fs}$ is a reasonable guess for systems containing light atoms like hydrogen, but may be adjusted due to temperature or rapid changes in the potential. It should always be about an order of magnitude smaller than the timescale of the fastest process in the system. Third, atomic velocities and the initial geometry must be defined. Both define the outcome of the essentially deterministic trajectory calculation. One has to keep in mind though, that true determinism cannot always be achieved as different CPUs and compilers produce different round-off errors using floating-point arithmetic. This leads to an exponential divergence of two trajectories in phase-space that differ only by a small amount at any point in time and is called Lyapunov instability [73].

Deciding on the simulation cell size is similar to the considerations about the time step. The problem of a finite unit cell size can be solved by the use of periodic slab boundary conditions. This means that if an atom exits the simulation cell on one side, it enters simultaneously with the same velocity at the other side. In consequence, two atoms can never be further apart than half the cell size. A vector $\boldsymbol{r}_{i j}$ pointing from atom $i$ to atom $j$ can be folded into the simulation cell via

$$
\boldsymbol{r}_{i j}^{\mathrm{pbc}}=\underline{\underline{\boldsymbol{M}}}\left[\underline{\underline{\boldsymbol{M}}}^{-1} \boldsymbol{r}_{i j}-\operatorname{nint}\left(\underline{\underline{\boldsymbol{M}}}^{-1} \boldsymbol{r}_{i j}\right)\right]
$$

with $\underline{\underline{M}}$ as the cell matrix and the nint-function rounds every vector element to the nearest integer. Then the minimum image convention is applied, meaning that atom $i$ only interacts with the nearest image of atom $j$ instead of all images [74]. For run-time reasons, we want the simulation cell to be as small as possible. Too small of a cell, however, can lead to unphysical behavior of the system. If the interaction between two atoms extends over a distance larger than half the cell size, then the minimum image convention prevents the calculation of the correct number of interacting pairs. Furthermore, phonon wavelengths are restricted to integer multiples of the cell dimension. This artificial discretization is inevitable, but larger cells of course enhance the phonon spectrum. 


\subsubsection{Newtonian Dynamics}

To simulate the evolution of the system over time, we assume that the nuclei are heavy enough to be treated as classical particles. Classical mechanics tells us that there is a connection between the potential function $V(\boldsymbol{r})$ and the forces acting on an object of mass $m$ which moves inside this potential

$$
\boldsymbol{F}=-\frac{\partial V(\boldsymbol{r})}{\partial \boldsymbol{r}}=m \frac{\mathrm{d}^{2} \boldsymbol{r}}{\mathrm{d} t^{2}}=m \boldsymbol{a}
$$

$\boldsymbol{r}$, again, is the set of all atomic coordinates in the system. As explained in the previous paragraph, we have to discretize the system in the time domain in increments of the time step $\Delta t$. Then the system can be propagated in steps of $\Delta t$ using a Taylor expansion to move from $\boldsymbol{r}_{i}$ to $\boldsymbol{r}_{i+1}$

$$
\begin{aligned}
\boldsymbol{r}_{i+1} & =\boldsymbol{r}_{i}+\frac{\mathrm{d} \boldsymbol{r}_{i}}{\mathrm{~d} t}(\Delta t)+\frac{1}{2} \frac{\mathrm{d}^{2} \boldsymbol{r}_{i}}{\mathrm{~d} t^{2}}(\Delta t)^{2}+\frac{1}{6} \frac{\mathrm{d}^{3} \boldsymbol{r}_{i}}{\mathrm{~d} t^{3}}(\Delta t)^{3}+\ldots \\
& =\boldsymbol{r}_{i}+\boldsymbol{v}_{i}(\Delta t)+\boldsymbol{a}_{i}(\Delta t)^{2}+\boldsymbol{b}_{i}(\Delta t)^{3}+\ldots
\end{aligned}
$$

where $\boldsymbol{v}, \boldsymbol{a}$ and $\boldsymbol{b}$ are the first, second and third derivatives of the position with respect to time, also known as velocity, acceleration and jerk. Several algorithms have been designed to integrate Eq. 2.35 numerically using the Taylor series. Euler and Runge-Kutta methods can be used for MD simulations, but one needs to keep in mind that they are not time-reversible. The Verlet algorithm [75] can in principle be used, but it does not consider velocities explicitly thus making thermostatting (section 2.4.4) difficult, and it is prone to numerical errors. The leap-frog algorithm does not suffer from these shortcomings, but positions and velocities are out of phase by half a time step. To obtain both at a given time, one would have to interpolate which adds unwanted complexity. This is the reason why the velocity Verlet algorithm was chosen for the MD simulations in this work. The iterative scheme is given by

$$
\begin{aligned}
\boldsymbol{v}_{i+\frac{1}{2}} & =\boldsymbol{v}_{i}+\frac{1}{2} \boldsymbol{a}_{i}(\Delta t) \\
\boldsymbol{r}_{i+1} & =\boldsymbol{r}_{i}+\boldsymbol{v}_{i+\frac{1}{2}}(\Delta t) \\
\boldsymbol{a}_{i+1} & =-\frac{\mathrm{d} V\left(\boldsymbol{r}_{i+1}\right)}{\mathrm{d} \boldsymbol{r}_{i+1}} / m \\
\boldsymbol{v}_{i+1} & =\boldsymbol{v}_{i+\frac{1}{2}}+\frac{1}{2} \boldsymbol{a}_{i+1}(\Delta t)
\end{aligned}
$$


which update the velocities by half a time step twice in one iteration. It is timereversible and symplectic (conserving phase-space volume) making it very robust in MD simulations while being straightforward to implement.

\subsubsection{Langevin Dynamics}

Langevin dynamics, expressed by

$$
m \boldsymbol{a}=-\frac{\partial V(\boldsymbol{r})}{\partial \boldsymbol{r}}-m \eta \boldsymbol{v}+\boldsymbol{F}^{\mathrm{st}}
$$

uses in principle the same equations as Newtonian dynamics with the exception that the calculation of the acceleration is not only based on the derivative of the potential, but also on two more terms. One of them is a friction term $-m \eta \boldsymbol{v}$ which decelerates a particle proportionally to its velocity with a factor of $\eta$. This parameter can be obtained in a number of different ways depending on the application. In the case of a hydrodynamical particle, it can be related to the particle radius, thereby introducing the notion of viscosity. In consequence, it is frequently used in solvation dynamics calculations based on the Navier-Stokes or Basset-Boussinesq-Oseen equations.

In the frame of scattering processes on metal surfaces, $\eta$ assumes the role of mapping the electron density onto a frictional force. Since metal surfaces are zero band gap materials, arbitrary amounts of energy can lead to electron-hole pair (ehp) excitation. The friction force can now model this continuously excitable energetic range by dissipating kinetic energy from the incoming particle into ehps. It is assumed that the PES does not change in the process because the dissipated energy per unit time is small in comparison with the electron diffusivity in a metal. This means that the coupling is weak between the projectile's translational and the metal's electronic DOFs and that there are no memory effects involved. These ideas are the basis of the Local Density Friction Approximation (LDFA) [76] which has been used throughout this work to model nonadiabatic effects. It is a well understood method and has been used recently to model vibrational de-excitation [77], dissociative adsorption [76] and vibrational lifetimes [78] of small molecules on metal surfaces, among many more.

The other term in Eq. 2.42 describes a stochastic force $\boldsymbol{F}^{\text {st }}$ that acts on a particle. In the hydrodynamical picture, it simulates random collisions with solvent molecules. In our surface collision simulations, it models the influence of the electronic temperature of the metal states on the incident particle. $\boldsymbol{F}^{\text {st }}$ has to obey several laws of physics that determine in which way the randomness influences the 
particles' motion. First, it has to obey the fluctuation-dissipation theorem, which is a very general concept relating small perturbations to statistical fluctuation at equilibrium. In the case of a single particle, it is defined as

$$
\left\langle\boldsymbol{F}^{\mathrm{st}}(t) \boldsymbol{F}^{\mathrm{st}}\left(t^{\prime}\right)\right\rangle=k_{\mathrm{B}} T_{\mathrm{el}} m \eta \delta\left(t-t^{\prime}\right) \hat{\boldsymbol{e}}
$$

with $k_{\mathrm{B}}$ as the Boltzmann constant, $T_{\mathrm{el}}$ as the electronic temperature, $\delta$ as the Dirac delta function and $\hat{\boldsymbol{e}}$ as the identity tensor. The magnitude of the random force is given by

$$
\boldsymbol{F}^{\mathrm{st}}(t)=\boldsymbol{\xi}(t) \sqrt{\pi \hbar k_{\mathrm{B}} T_{\mathrm{el}} m \eta}
$$

in which the time dependence arises from the stochastic process $\boldsymbol{\xi}(t)$. Each component $\xi_{i}(t)$ is drawn from a standard normal distribution 'on the fly' during the simulation. The Dirac delta function in Eq. 2.43 points out that subsequent drawings from the distribution are uncorrelated. These equations can also be used to thermostat a system towards $T_{\mathrm{el}}$.

The only remaining question is how to obtain the friction coefficient $\eta$ from the electron density at the surface and inside the metal bulk. Since EMT (section 2.3.1) is based on the UEG, it is reasonable to use the same system to gather information about $\eta$. Puska and Nieminen used DFT at the LDA level to calculate wave function shifts of an electron scattering from a nucleus which was embedded in the UEG [79]. The stopping power that the electron experienced could subsequently be converted into a friction coefficient that would have caused the same shift. Following previous efforts in the Wodtke group [80], the connection between the friction coefficient and the electron density from EMT (Eq.2.18), based on Puska's and Nieminen's data, is parametrized by

$$
\hbar \eta(n)= \begin{cases}\sum_{i=1}^{12} c_{i} n^{i} & n \leq 0.36 \AA^{-3} \\ d_{1}-\mathrm{e}^{-d_{2} n} \cdot 10^{-3} \mathrm{meV} & n>0.36 \AA^{-3}\end{cases}
$$

and the expansion coefficients are provided in Tab. 1.

\subsubsection{Ring Polymer Molecular Dynamics}

Classical MD lacks the ability to capture quantum effects in a simulation. The importance of quantum effects is of course strongly system-dependent in terms of 
Table 1: Expansion coefficients used in Eq. 2.45 to calculate the electronic friction. The unit of $c_{i}$ is $\mathrm{meV} \cdot \AA^{3 i}$.

\begin{tabular}{llll}
\hline$c_{1}$ & 0.0802484 & $c_{2}$ & -1.12851 \\
$c_{3}$ & 9.28508 & $c_{4}$ & 2.10064 \\
$c_{5}$ & -843.419 & $c_{6}$ & 8853.54 \\
$c_{7}$ & -48902.3 & $c_{8}$ & 167410 \\
$c_{9}$ & -367098 & $c_{10}$ & 503476 \\
$c_{11}$ & -394260 & $c_{12}$ & 134763 \\
$d_{1}$ & $0.0047131 \mathrm{meV}$ & $d_{2}$ & $4.41305 \AA^{3}$ \\
\hline
\end{tabular}

the involved masses or given temperature. Since this work investigates hydrogen atom scattering, treating the system purely classically runs the risk of neglecting important features in the scattering distribution due to quantum effects. It is known for example that tunneling can increase the rate of a proton transfer reaction by several orders of magnitude [81].

Craig and Manolopoulos introduced Ring Polymer Molecular Dynamics (RPMD) in 2004 which aims at modeling tunneling and zero point energy effects in classical MD simulations [82]. They used the imaginary-time path integral formalism to approximate quantum effects by using techniques from classical MD only. It is reasonable to do so because a classical ring polymer is isomorphic to a quantum particle in the limit of an infinite number of beads. Time savings compared with the full quantum treatment stem from the fact that real-time quantum coherence is neglected. If coherence vanishes quickly like in condensed matter either due to thermal averaging or strong intermode coupling, RPMD can prove as a valuable method. Within the RPMD framework, atoms are represented by necklaces having a certain number of beads, $P$, which are connected via harmonic springs. A trajectory runs on the same PES as classical MD, but exists in an extended phase space. It yields correct quantum mechanical results in the high-temperature and short-time limit. This section can only provide some key concepts and equations. For a recent review about RPMD see Ref. [83], and detailed introduction about path integrals can be found in Ref. [84]. 
The Hamiltonian in RPMD is given by

$\hat{\mathcal{H}}(\boldsymbol{p}, \boldsymbol{x})=\sum_{s=1}^{P}\left\{V\left(\boldsymbol{x}_{i}^{(s)}, \ldots, \boldsymbol{x}_{N}^{(s)}\right)+\sum_{i=1}^{N}\left[\frac{\left(\boldsymbol{p}_{i}^{(s)}\right)^{2}}{2 m_{i}}+\frac{m_{i}}{2}\left(\frac{P}{\beta \hbar}\right)^{2}\left(\boldsymbol{x}_{i}^{(s)}-\boldsymbol{x}_{i}^{(s-1)}\right)^{2}\right]\right\}$

where $V$ is the classical potential function that has to be evaluated $P$ times; once for every bead index $s$, and $\beta=\left(k_{\mathrm{B}} T\right)^{-1}$. As usual, the Hamiltonian contains the kinetic energy of all particles and one term which looks similar to the harmonic spring potential energy $\frac{1}{2} k\left(x-x_{0}\right)^{2}$. Indeed, if the system behaves classically either due to high temperatures or large atomic masses, the spring constant becomes large as well. In the limit of $\hbar \rightarrow 0$, the ring polymer shrinks to a single point recovering classical MD. This can also be seen from the radius of gyration of a free ring polymer [85]

$$
r_{\mathrm{G}}(\text { free })=\sqrt{\frac{\beta \hbar^{2}}{4 m}\left(1-\frac{1}{P^{2}}\right)}
$$

The equations of motion read

$$
\begin{aligned}
\frac{\mathrm{d} \boldsymbol{x}_{i}^{(s)}}{\mathrm{d} t} & =\frac{\partial \hat{\mathcal{H}}}{\partial \boldsymbol{p}_{i}^{(s)}}=\frac{\boldsymbol{p}_{i}^{(s)}}{m_{i}} \\
\frac{\mathrm{d} \boldsymbol{p}_{i}^{(s)}}{\mathrm{d} t} & =-\frac{\partial \hat{\mathcal{H}}}{\partial \boldsymbol{x}_{i}^{(s)}}=-m_{i}\left(\frac{P}{\beta \hbar}\right)^{2}\left(2 \boldsymbol{x}_{i}^{(s)}-\boldsymbol{x}_{i}^{(s-1)}-\boldsymbol{x}_{i}^{(s+1)}\right)-\frac{\partial V\left(\boldsymbol{x}_{1}^{(s)}, \ldots, \boldsymbol{x}_{N}^{(s)}\right)}{\partial \boldsymbol{x}_{i}^{(s)}} .
\end{aligned}
$$

These equations are useful to see what happens during the propagation of the ring polymer and how they are related to the Hamiltonian. In practice though, the springs between the beads can become really stiff. Then the simulation time step has to be very small due to the couplings between neighboring beads to ensure conservation of energy. A better representation of the ring polymer during the propagation step is to convert it to normal modes. One can take advantage of Fast Fourier Transform techniques for this purpose or use an analytical orthogonal matrix transformation. Since normal modes are orthogonal to one another, the propagation is exact for arbitrary time steps, independent of the force constant. The ring polymer propagation scheme in normal modes and the $P$-dimensional real 
discrete Fourier transform is described in detail in [86]. In equilibrium RPMD, all normal bead modes depend on the centroid mode which describes the movement of the ring polymer as a whole. In this case the normal mode energies can easily be found by using the Frost Circle scheme known from Hückel theory for aromatic compounds.

One of the most visible extension of classical MD can be seen on the basis of the kinetic energy expression. The virial estimator [87] reads

$$
E_{\text {kin }}=\frac{3}{2 \beta}+\frac{1}{2 N P} \sum_{i=1}^{N} \sum_{s=1}^{n}\left(\boldsymbol{x}_{i}^{(s)}-\overline{\boldsymbol{x}}_{i}\right) \frac{\partial V\left(\boldsymbol{x}_{1}^{(s)}, \ldots, \boldsymbol{x}_{N}^{(s)}\right)}{\partial \boldsymbol{x}_{i}^{(s)}}
$$

where the centroid $\overline{\boldsymbol{x}}_{i}$ is defined as the average over the beads of the $i^{\text {th }}$ ring polymer necklace

$$
\overline{\boldsymbol{x}}_{i}=\frac{1}{n} \sum_{s=1}^{P} \boldsymbol{x}_{i}^{(s)}
$$

The first term in Eq. 2.50 corresponds to the classical kinetic energy $\frac{1}{2} k_{\mathrm{B}} T$ per translational DOF. The second term describes the contribution to $E_{\text {kin }}$ due to quantum fluctuations. It vanishes if the ring polymer is not under the influence of an external field, but can contribute noticeably if condensed matter at low temperatures is simulated [85]. This is a direct consequence of the Heisenberg uncertainty principle. The more the ring polymer contracts due to external forces, the higher the bead kinetic energy. An increased localization directly leads to more fluctuations of the momenta [88]. The justification to use this approach for scattering simulation comes from a paper by Welsch et al. [89]. They found that if a particle is provided with an initial momentum impulse, RPMD still retains the formal connection to Matsubara dynamics (see section 3.4 for more details). In fact RPMD agrees with it up to $\mathcal{O}\left(t^{5}\right)$ for the position-autocorrelation function and performs equally well or even better than equilibrium RPMD in their simulations. Hence, the impinging particle in the scattering simulations which is clearly not in any equilibrium does not prevent one from using this method.

\subsubsection{Thermostats}

Thermostatting plays an important role in the MD simulations discussed in this thesis. Suppose there exists a suitable PES to describe the system of interest, 
the first step is to find the minimum energy configuration of the surface slab. A straightforward way to find the optimal geometry will be described in section 2.5.3. Starting with this optimized geometry, how can we infuse the correct amount of energy to heat the surface to the desired temperature? Three methods for this task shall be described here which were all used at one point or the other in this work.

The simplest method is what I like to call the method of equipartition. If we want the system to thermalize to $T$, we simply draw random numbers from a normal distribution $\mathcal{N}\left[0,\left(2 m k_{\mathrm{B}} T\right)^{-1}\right]$ for the three dimensions of the velocity vector of each atom individually. This situation corresponds to a Maxwell-Boltzmann (MB) speed distribution of $2 T$. The equipartition theorem states that the available energy in a system is distributed equally among all degrees of freedom. This means that for a set of coupled harmonic oscillators the average kinetic and potential energy of the system are equal. Since the initial geometry was chosen as the equilibrium situation where $E_{\text {pot }}$ equals zero, half of the kinetic energy will be converted to potential energy over time. This will leave the system at the desired temperature of $T$ after equilibration. The success of this line of actions depends on several criteria though. First, the geometry at equilibrium has to be known beforehand and second, the system needs to correspond to a set of coupled harmonic oscillators. This is usually the case if $T$ is well below the melting temperature $T_{\mathrm{m}}$ of the material. Finding the equilibrium geometry is straightforward, since the crystal systems are known for the considered materials. The second criterion depends on the system and the desired temperature of course. For example, thermalizing to room temperature with this method will work well for platinum $\left(T_{\mathrm{m}}^{\mathrm{Pt}}=2040 \mathrm{~K}\right)$, but certainly not for lead $\left(T_{\mathrm{m}}^{\mathrm{Pb}}=600 \mathrm{~K}\right)[90]$.

The next method, called the Andersen thermostat [91], also works by drawing random numbers from a MB distribution, but this time at the desired temperature $T$. The idea is to let a random atom in this system collide with an artificial bath from time to time. The bath always stays at a temperature of $T$. The collision is modeled by replacing a random velocity vector component of an atom by the one drawn from the MB distribution. Since the system is given enough time between the collisions to equilibrate, it is slowly heated to $T$. Starting from the equilibrium geometry is not required, since the temperature will be raised or damped to $T$ sooner or later. Of course, it should not deviate too much, especially when the system can form more than one stable structure.

The last thermostatting technique is called Path Integral Langevin Equation (PILE) and works especially well for RPMD simulations [86]. For classical MD, 
it reduces to the Langevin equations of motion (cf. section 2.4.2). The PILE momentum update in an RPMD trajectory consists of the following three steps: first, the momentum of the beads is transformed from Cartesian coordinates $j$ to normal modes $k$ using an orthogonal transformation matrix $\underline{\underline{C}}_{j k}$. Next, a friction and a random force are applied to each normal mode of the ring polymer. $\gamma(k)$ is the friction coefficient on the normal mode $k$ and $\boldsymbol{\xi}^{(k)}$ is a vector of independent Gaussian numbers, just like in Eq. 2.44. Last, the coordinates are transformed back to Cartesian coordinates.

$$
\begin{aligned}
\tilde{\boldsymbol{p}}^{(k)} & \leftarrow \sum_{j=1}^{n} \boldsymbol{p}^{(j)} \underline{\underline{\boldsymbol{C}}}_{j k} \\
\tilde{\boldsymbol{p}}^{(k)} & \leftarrow \mathrm{e}^{-\frac{\Delta t}{2} \gamma^{(k)}} \tilde{\boldsymbol{p}}^{(k)}+\sqrt{\frac{m_{i}}{P k_{\mathrm{B}} T}\left(1-\mathrm{e}^{-\Delta t \gamma^{(k)}}\right)} \boldsymbol{\xi}^{(k)} \\
\tilde{\boldsymbol{p}}^{(j)} & \leftarrow \sum_{k=0}^{n-1} \underline{\underline{\boldsymbol{C}}}_{j k} \tilde{\boldsymbol{p}}^{(k)}
\end{aligned}
$$

The most efficient friction coefficients for the ring polymer normal modes can be found analytically by minimizing the autocorrelation time of the harmonic oscillator Hamiltonian [86]. Only the friction coefficient of the centroid mode, describing the movement of a ring polymer as a whole, needs be set manually. This is similar to the time between particle-bath collisions of the Andersen thermostat. A value too low will result in wild oscillations of the temperature, and simulations with a value too high will take a long time to converge.

\subsection{Optimization Algorithms}

Various optimization techniques have been applied throughout this work. First and foremost, for the parametrization of the EMT potentials that can describe a hydrogen atom at a metal surface, I used an approach to global optimization (section 2.5.2) in combination with a local one (section 2.5.1). The REBO PES was fitted entirely using local optimization because of the much higher computational demands. section 2.5.3 describes a procedure by which the equilibrium geometry can be found if a fairly reasonable initial guess is available. Optimization also plays a role in the trajectory analysis when looking for incidence conditions that lead to a maximum sticking probability or scattering angles of maximum intensity. 


\subsubsection{Trust Region}

The Trust Region (TR) algorithm is an extension to the Levenberg-Marquardt (LM) scheme $[92,93]$ to solve a system of nonlinear equations. It is based on the idea that real optimization problems can be replaced by a model function, usually obtained in a Taylor-like fashion, but other forms are possible [94]. This model function is dramatically less complex making optimization much simpler. As in every Taylor expansion, the model function describes the approximated function best around the point used in the expansion. Hence, the algorithm got its name from the region in which the model function can be trusted to be a good representation of the actual problem.

Using least squares to measure the deviation of the fitted function $f(\boldsymbol{x})$ from the reference data $\boldsymbol{y}$, the problem to solve can be formulated as

$$
\min _{x \in \mathfrak{R}^{n}}\|F(\boldsymbol{x})\|_{2}^{2}=\min _{x \in \mathfrak{R}^{n}}\|\boldsymbol{y}-f(\boldsymbol{x})\|_{2}^{2}, \boldsymbol{x} \in \mathfrak{R}^{n}, \boldsymbol{y} \in \mathfrak{R}^{m}, f: \mathfrak{R}^{n} \rightarrow \mathfrak{R}^{m}, m \geq n .
$$

$m$ describes the number of data points, $n$ is the number of independent variables of $f(\boldsymbol{x})$, i.e., the number of parameters of the PES. $f(\boldsymbol{x})$ must be twice differentiable in $\mathfrak{R}^{n}$ and $\|\cdot\|_{2}$ is the $\ell_{2}$ norm. Starting from a parameter set $\boldsymbol{x}_{i}$, the optimized set $\boldsymbol{x}_{i+1}$ after one step $\boldsymbol{s}$ is obtained via

$$
\min _{x \in \Re^{n}}\left\|F\left(\boldsymbol{x}_{i}\right)+J\left(\boldsymbol{x}_{i}\right)\left(\boldsymbol{x}_{i+1}-\boldsymbol{x}_{i}\right)\right\|_{2}^{2} \quad \text { subject to } \quad\left\|\boldsymbol{x}_{i+1}-\boldsymbol{x}_{i}\right\| \leq \boldsymbol{\Delta}_{i},
$$

where $J$ is the Jacobian of $F$ and $\boldsymbol{\Delta}$ defines the edge of the TR which no $s$ may exceed [95]. The update rules for $\boldsymbol{\Delta}$ are based on the ratio between actual and predicted improvement of $F$ and can be found among other details in Ref. [96].

One problem with the LM scheme is that if the Hessian becomes negative definite (a negative curvature of the objective function is encountered), the algorithm slows down considerably. The TR algorithm on the other hand chooses $\boldsymbol{\Delta}$ in a way that a large step can be performed in such a case. Consequently, parameter space can move quickly from negative curvature regions towards the desired minimum of the objective function. This behavior is especially desired when fitting a PES. The computation of the objective function is by far the most time-consuming step. Therefore, the higher computational complexity of the TR compared to the LM algorithm is worth the effort if a few iterations during optimization can be saved. [97] 


\subsubsection{Genetic Algorithm}

Genetic Algorithms (GAs) are based on Charles Darwin's theory of natural selection [98] and modern advances in genetics research. They use selection, recombination and mutation to mimic the efficiency and flexibility of biological systems. No information about continuity or differentiability of the objective function are required. The phrase 'survival of the fittest' in this context refers to candidate solutions which form a population. Each individual solution is an approach to solving the problem in a different way. How these solutions are coded into the individuals can vary substantially depending on the problem. In any case, the genetic markup, also called the genotype, must allow for the definition of crossover and mutation operators that advance one generation to the next. Also, there must be an objective function that evaluates each candidate solution and assigns it a fitness value. After all creatures have been rated, the selection operator uses each individual's score to assign it a crossover probability. In the last step, two individuals are successively selected to crossover and form two new members of the next generation until the new population has reached the size of the old one. [99]

The encoding of the individuals plays an important role in the optimization task. In general, it consists of a fixed-length string, called a chromosome, using a certain alphabet. In this work, floating point numbers make up the alphabet, but one can also imagine using integers to indicate game piece positions on a board game, for example. For explanatory purposes, I will use the binary encoding to illustrate the key concepts and operators that can be found in every GA implementation. Following the terminology from biology, a certain position on the chromosome is called a gene, and the value that a gene carries is called an allele. Regarding the binary encoding, * represents the wildcard character for the allele zero or one. Among other things, GAs are so successful because they can connect schemata in the encoding with the objective function. A good schema is also called a building block, since valuable individuals are made up of these blocks strung together. Building blocks can also be identified by a high score in the objective function and by a small distance between the delimiting alleles (Hamming distance). High Hamming distance building blocks cannot exist over multiple generations, because the crossover operator has a high tendency to separate them. Of course, if the chromosome is cut at a random position, the scheme $\left[{ }^{*} 101^{* * * *}\right]$ has a higher survival probability than $\left[{ }^{*} 1^{* *} 0^{* *} 1\right]$.

The first step in a simple GA is to select two individuals which recombine to produce two new, potentially improved ones. To do so, there has to be a way to assess 


$$
\begin{array}{r}
\text { Generation } i:[00101 \mid 011]+[11101 \mid 001] \\
\text { Generation } i+1:[00101 \mid 001]+[11101 \mid 011]
\end{array}
$$

Figure 2: Single-point crossover of two 8-bit strings. The crossover point is marked by a vertical bar.

the fitness of each individual. Suppose there exists a reasonable fitness function, then one can use roulette wheel selection. Illustratively speaking, each creature is assigned a share of a roulette wheel proportional to its fitness. The rotation of the wheel together with the spinning ball then pick a creature for procreation at random. This is a simulation of natural selection, where stronger and healthier creatures have a higher probability to reproduce. Other selection criteria are also possible. One can imagine to only select one creature based on the fitness and the other at random. This strategy can be used to maintain diversity if otherwise the population would be dominated by a few extremely good individuals. Alternatively, a nonlinear selection method can be applied to counteract premature convergence. [99, 100]

Just like with the selection rules, there exist numerous ways in which reproduction in a GA can take place. The main point is that it enables information exchange between individuals within a generation, which is illustrated in Fig. 2. At first, a position on the chromosome, called the locus, is chosen at random. Then both parent chromosomes are cut at this locus and exchange parts of their genetic material. Hence, they produce two children which belong to the subsequent generation. Other methods of crossover include, but are not limited to: two- or $k$-point crossover where two or $k$ loci are randomly chosen, uniform crossover where each gene of the child's chromosome is randomly taken from a parent or ordered-lists crossover if single-point crossover would lead to too many invalid solutions. Using more than two parents, although not inspired by nature, is of course also possible and may lead to better results [101].

The mutation operator is applied to the population after crossover. It is the last step in the life cycle of a generation. Mutation is also inspired by nature, and although it often leads to negative results in real world creatures, positive effects have also been observed [102]. Natural selection subsequently determines the dissemination of the mutated gene. In a GA, mutation is realized by replacing one allele with a different one from the available alphabet. This procedure is called single point mutation if it happens independently from other genes in the chromosome. If we take the binary string genotype representation again as an example, mutation is most easily performed by applying the logical XOR operator. One bit pattern is 
[00101011] Figure 3: The top bit string is the creature undergoing mutation. The bit XOR [00010010] pattern in the middle defines the loci where mutation in form of a bit flip takes

[00111001] place. The resulting creature on the bottom was generated by performing the XOR operation on each pair of corresponding bits.

the creature undergoing mutation and the other a string of the same length with ones in the positions to be mutated, as shown in Fig. 3. The purpose of mutation is, again, to ensure genetic diversity over all populations. If no mutation took place, the explorable search space would be entirely determined by combinations of the initial population. Simply by chance, good building blocks might be destroyed by recombination and there would be no way to rediscover them. Likewise mutation might introduce building blocks that the initial population does not contain. The mutation probability is usually set to a small number and the efficiency strongly depends on the optimization problem and on the other operators. But it is clear that a high mutation rate hinders convergence since it easily turns the GA into a random search. [103]

GAs are of course not the best solution to all global optimization problems. Other methods like simulated annealing, particle swarm optimization or linear programming can be used as well. A computationally very expensive fitness function for example prohibits the use of a GA. It might well happen that the fitness function evaluation takes half a day. So to use it in a GA, this function would have to be computed for a few hundred individuals over a few hundred generations. In this case a GA would simply take too long to come up with reasonable solutions. There might also be problems if the dimensionality of search space is very high. Then even if one uses large populations, the trial solutions will still be very sparsely distributed in the search domain. In problems without a reference fitness, it can be difficult to estimate the quality of the solutions, since only relative improvements in the fitness can be observed during run time. In consequence, the formulation of a stop criterion is not obvious because there is no way to check if the global minimum has been found. The probability of getting stuck in a local minimum is strongly dependent on the fitness landscape [104]. And since there is no mechanism in a GA to sacrifice short term fitness to gain more in the long run, the only way to optimize the performance is to tune hyperparameters like the crossover operator or mutation probability. Ironically, this task itself is an optimization problem, but at least we know that there is no general solution to it [105]. The only condition that always helps in obtaining good solutions it to make sure that the populations contain diverse creatures. One possibility would be to simply replace a duplicate with 
a random solution, or less invasively, penalize creatures that have agglomerated in a niche in search space.

To summarize, randomness is a recurrent theme in GAs because it promotes information exchange between individuals and ensures diversity. This way new individuals speculate on worthwhile points in search space that can improve the results. Since there is always a population trying to find a good solution instead of a single point, the algorithm runs inherently in parallel. All of these properties enable a GA to solve global optimization problems efficiently. The reason seems to be that, similar to discoveries made my mankind, it is necessary to combine different ideas to arrive at a good solution. Though a discovery is seldom the result of pure luck, some degree of serendipity often plays an important role. GAs tie in with this concept and can come up with new solutions from the best constituents of previous tries. [99]

\subsubsection{Fast Inertial Relaxation Engine}

The equilibrium geometry of the simulated system is of crucial importance. Since it is only defined by the PES, lattice parameters, bond lengths and adsorption energies can be easily extracted and compared with other methods. It is also the starting point for a very simple thermalization method (cf. section 2.4.4). In general, the determination of the minimum energy structure belongs to the class of NPhard problems [106], since it is believed that the number of local minima grows exponentially with the dimensionality [107]. Global optimization problems of this kind are best tackled by stochastic-heuristic methods like GAs coupled with gradient descent [100].

Luckily in this work, the overall structure of the materials is known from the literature. This means for metal surfaces for example, the bulk lattice constant can be directly obtained from the PES parameters. Most of the time, the $3+3$ approximation [108] to the slab was used in the simulations. The bottom three atomic layers are fixed to the equilibrium separation to simulate the bulk while the top three layers are allowed to relax.

Since we can be sure to start from a good initial guess to the equilibrium structure, a simple local optimization strategy will suffice. A particularly efficient scheme for this purpose was developed by Bitzek et al. called the Fast Inertial Relaxation Engine (FIRE) [109]. It is based on the damped molecular dynamics approach with velocity modification and makes use of an adaptive time step to speed up the op- 
timization. The MD steps can be performed with any integration scheme for the equations of motion. FIRE outperforms conjugate gradient methods and is comparable to the L-BFGS algorithm in terms of run time, but has less computational overhead and requires less memory. The idea is to add an inertia term in the calculation of the acceleration

$$
\boldsymbol{a}(t)=\frac{\boldsymbol{F}(t)}{m}-\gamma(t)|\boldsymbol{v}(t)|[\hat{\boldsymbol{v}}(t)-\hat{\boldsymbol{F}}(t)]
$$

where hats indicate unit vectors and $\gamma(t)$ determines the amount of mixed-in inertia. The power $P(t)=\boldsymbol{F}(t) \boldsymbol{v}(t)$ is used for time step adaptation. If it is positive (magnitude-weighted velocity and force vectors differ by less than $90^{\circ}$ on average) during multiple time steps, the time step and the contribution of the inertia is increased. As soon as the power becomes negative, $\boldsymbol{v}$ is set to zero, the time step is decreased and $\gamma$ is set to the initial small value.

There are seven parameters in this algorithm: the number of steps after which the time step is increased, the increase and decrease rates of the time step, the initial value and increment of the inertia contribution and the initial and the maximum time step. Robust parameters together with all details about the algorithm can be found in [109]. In general, no parameters need to be set by hand. Since it is not real $\mathrm{MD}$, all masses in the system can be arbitrarily set to $1 \mathrm{u}$ to treat all degrees of freedom equally. Then we know that $1 \mathrm{fs}$ is a reasonable guess for the initial time step. 


\section{Methods}

\subsection{VASP}

The Vienna ab initio Simulation Package (VASP) [110-114] was used throughout this work for several reasons. First, Density Functional Theory (DFT) software which uses plane waves is clearly at an advantage compared to atom-centered basis functions for the description of surfaces. Plane waves natively fulfill the Bloch theorem [115] meaning the description of the first Brillouin zone is sufficient to model the entire surface. Second, the package employs pseudopotentials as a means for reducing the basis set size, so that only the outermost electrons are treated explicitly in the calculations. Especially for heavy atoms, this approximation is a source of great savings in computation time. Third, single-particle Kohn-Sham (KS) orbitals of high principle quantum numbers show large oscillations near the atomic nucleus. To avoid calculating the wave function on a very dense grid to capture their rapidly changing character, VASP utilizes the Projector Augmented Wave Method [116] to smoothen the function near the core. This reduces the density of the Fourier transform grid in reciprocal space, further accelerating calculations. Much more detailed information about the software package can be found in the VASP manual [117].

The input to VASP consists of four files. The INCAR file contains all the input keywords and flags and determines what kind of calculation should be performed. KPOINTS specifies distinct points in $k$-space which replace the integral over the entire irreducible Brillouin zone during the calculation. POSCAR contains the cell vectors and positions of the atoms and POTCAR contains all pseudopotential coefficients. VASP in version 5.3.5 was used in this work to calculate the interaction of a hydrogen atom with face-centered cubic (fcc) transition metal surfaces. These energies were subsequently used to fit an Effective Medium Theory (EMT) Potential Energy Surface (PES). The fitting was done with the 'md_tian' program (section 3.2) which was called by the 'Skycruiser' genetic algorithm wrapper script (section 3.3). The procedure for generating DFT reference data using VASP is described in section 4.1.1.

\section{$3.2 \mathrm{md}$ tian}

md_tian was the first Molecular Dynamics (MD) program written in this group by Svenja M. Janke, Sascha Kandratsenka and Daniel J. Auerbach and was maintained 
until 2016. It was written in Fortran and implements Newtonian and Langevin dynamics for the simulation of $\mathrm{H}$ on gold using a full-dimensional EMT PES. md_tian can also be used to fit the PES to ab initio data via the nonlinear least squares Levenberg-Marquardt approach.

I extended the program by first replacing the Fortran source file responsible for fitting. The collection of subroutines was originally written on punched cards in the late sixties and later transcribed to source code. As a result, most variable names carry no meaning. The excessive use of computed GoTos makes it close to impossible to understand and extend. It heavily relies on data statements and common blocks which are outdated by modern programming standards.

The legacy source file was replaced by a Fortran module that implements the trust region nonlinear least squares algorithm (cf. section 2.5.1) with linear bound constraints from the Intel Math Kernel Library [95]. It works towards locally minimizing the vector of energy differences squared between some DFT reference energies and the energies predicted by the PES for the same atomic geometries. The PES parameters act as the independent variables in the energy function and are adjusted by the fitting algorithm. Boundaries can be set for each parameter to limit search space and to prevent possible unphysical behavior of the fitted PES. Multiple regression tests were performed to ensure that the new module produces the same or better results than the former collection of subroutines.

md_tian was primarily designed to simulate scattering experiments of $\mathrm{H}$ from a gold surface. Unfortunately, this scope of application was too narrow for upcoming experiments in this group. Many constants, the input and output routines and the general setup of the program were focused on gold, but O. Bünermann et al. were planning on scattering $\mathrm{H}$ atoms from many more fcc-metal surfaces namely $\mathrm{Pt}, \mathrm{Ag}, \mathrm{Pd}, \mathrm{Cu}$ and $\mathrm{Ni}$. Just as theory had provided valuable insights into the scattering dynamics before [58], I wanted to model and understand the outcomes of these experiments using the methodology described in section 3.1. Hence, I rewrote parts of the program to be as generic as possible with respect to the atomic surface elements. Other changes included improvements to the direct space representation of the system, enhancement of modularization, restriction of variable scopes and minor bugfixes. 


\subsection{Skycruiser}

Though the basic methodology for fitting an EMT PES had been established by S. M. Janke, the actual tuning of the parameters turned out to be much like an undirected search. In EMT, each atomic species is characterized by seven parameters which determine the interaction between atoms of the same type. Interactions between different atomic types are described by parameter mixing rules similar to the Lennard-Jones (LJ) potential. Therefore, the H-Au parameter space consists of 14 parameters in total. Three parameters of the surface type can be held fixed which determine the bulk modulus $B^{\mathrm{Au}}$. For gold, they read $E_{0}^{\mathrm{Au}}, \lambda^{\mathrm{Au}}$ and $s_{0}^{\mathrm{Au}}$. The latter can be obtained from the lattice constant of the $a b$ initio calculations and the cohesive energy $E_{0}^{\mathrm{Au}}$ can be found in the literature. Literature values also exist for $B^{\mathrm{Au}}$ which in turn determines $\lambda^{\mathrm{Au}}$. Still, it is very difficult to find the global minimum in an 11-dimensional space. Plus, even if it is found, there is no feasible way to prove it.

It is ensured that the fitting subroutine will find the nearest minimum starting from the initial parameters. But first, nobody can say what the Root Mean Square Error (RMSE) to the training data will be in that minimum and second, a small RMSE does not automatically mean that the adjusted parameter set produces a physically reasonable PES. One problem encountered in the past is that an $\mathrm{H}$ atom may pull a gold atom out of the surface and form an $\mathrm{H}-\mathrm{Au}$ diatomic in the vacuum region. Hence after a fit, it must be checked that $\zeta$, which is the difference between the metal cohesive energy and H-metal binding energy, is negative and preferably close to literature values. Another quantity to check is the melting temperature. It is definitely proportional to the cohesive energy, but also depends on all other parameters. One has no control over the evolution of the PES once the optimization starts, since it is strictly proceeding downhill on the RMSE landscape.

In her dissertation, Janke set the initial parameters of over one thousand fits manually to arrive at a PES that has a low RMSE, and fulfills the secondary fit criteria [80]. Of course, generating at least five more PESs for $\mathrm{H}$ on other fcc metals with this approach is not an option. Hence, I decided to design a wrapper script for md_tian called Skycruiser that is able to mix fitted PESs via the Genetic Algorithm (GA) approach detailed in section 2.5.2. It is written in Python since runtime spent in the wrapper is negligible compared to the computationally intensive energy computations done with md_tian. The GA can run in parallel by creating multiple instances of md_tian which all perform a different local optimization. It 
creates a synchronization barrier between subsequent generations. After all threads have arrived at the barrier, the roulette wheel selection operator is applied to all PESs, followed by crossover and mutation. The same steps are reapplied to the new PESs created this way and the algorithm stops when the average fitness levels off. The functional principle has already led to a publication [118], but will also be explained in the next paragraphs.

An individual in this GA does not only describe the PES, but also contains a recipe for md_tian about how the local fit shall be performed. There is, of course, one chromosome that holds the initial 14 EMT parameters. The first generation derives their parameters from the literature [57]. Usually, it is not problematic that all creatures start at the same point in parameter space as shall be explained shortly. There is an option in Skycruiser though that applies a Gaussian blur to the parameter set of the first generation. The second chromosome is binary and also has a length of 14 . The boolean values determine whether the allele in the parameter chromosome at the same index is subject to local optimization in the next call to md_tian. This already enables a creature to evolve in $2^{11}$ different directions in the first local optimization step, since three parameters are fixed to reproduce the bulk modulus, cohesive energy and ab initio lattice constant. An individual is also assigned an ab initio Molecular Dynamics (AIMD) trajectory of which there are about a dozen to choose from. Not all geometry-energy pairs which define that AIMD trajectory are used as training data though, since configuration space does not change much from one MD step to another. Such redundancies should be avoided because they introduce tight clusters in phase space. A clear indication for problematic phase space sampling is great accuracy on the training data, but a bad prediction of validation data. Hence, every individual is created with an AIMD contribution between 0-100\%. A percentage-based value is required here, because not all AIMD trajectories are of equal length. The AIMD contribution determines what percentage of geometry-energy pairs from the trajectory are used for training the PES in addition to the aforementioned DFT grid. The other AIMD trajectories are used in their entirety as validation data for each individual.

Next, an instance of the md_tian program is created for each individual with the setup that it contains. Skycruiser uses a queue system that facilitates massive parallelization since each local optimization is independent of all others. After the parameter set of all individuals was optimized, the resulting training and validation RMSEs are read back into Skycruiser and each individual receives a new chromosome with the optimized parameters. Then, the evaluation of the fitness takes place. The 
basis for the fitness $F$ is a mixture of training (td) and validation (vd) RMSE, given by

$$
F \propto \frac{\alpha}{\mathrm{RMSE}_{\mathrm{vd}}}+\frac{1-\alpha}{\mathrm{RMSE}_{\mathrm{td}}}
$$

$\alpha$ serves as a weighting factor and is usually set to 0.5 . One key design concept that decisively contributes to the success of this GA approach is the inclusion of secondary fit criteria into the fitness value. As explained before, the RMSE is not the only trait determining the goodness of a fit. Therefore, penalties are applied to the fitness in case the metal-H bond energy or metal shear elastic modulus deviates too far from their respective literature value. The penalty can also be applied to one or more parameters directly should one observe that any of them tends to assume an absurd value over the generations. The penalty function $P_{k}$ for attribute $k$ is given by

$$
P_{k}\left(x_{k}\right)=p_{k}\left(\left|\frac{x_{k}}{x_{k}^{0}}-1\right|-\Delta_{k}\right)
$$

where $x_{k}$ is the value of $k$ that might be penalized, $x_{k}^{0}$ is the target or literature value, $p_{k}$ defines the penalty slope and $\Delta_{k}$ defines a penalty-free region around $x_{k}^{0} . P_{k}\left(x_{k}\right)$ has its roots at $x_{k}^{0} \pm \Delta_{k}$, is negative inside this range and strictly increases outside of it. By how much the penalty increases for each additional percent of deviation from $x_{k}^{0}$ can be determined by $p_{k}$. It usually involves some try and error to find a penalty slope that works well because it needs to be harmonized with the fitness values that the population can reach for this task. To prevent the penalty from becoming negative and actually increasing the fitness, a final penalty $P$ is computed by

$$
P=\sum_{k} \max \left[0, P_{k}\left(x_{k}\right)\right]
$$

that sums up only positive contributions. Finally, this value is subtracted from the fitness computed via Eq. 3.1 and each creature is assigned its final fitness.

In the crossover step, the GA selects individuals with a probability proportionate to their fitness. This roulette wheel selection algorithm is displayed in Fig. 4 and is called twice to determine two parents. In case the function returns the same index both times, it is called until the second index differs from the first to prohibit cloning. The recombination operator then proceeds as follows: first, a number 


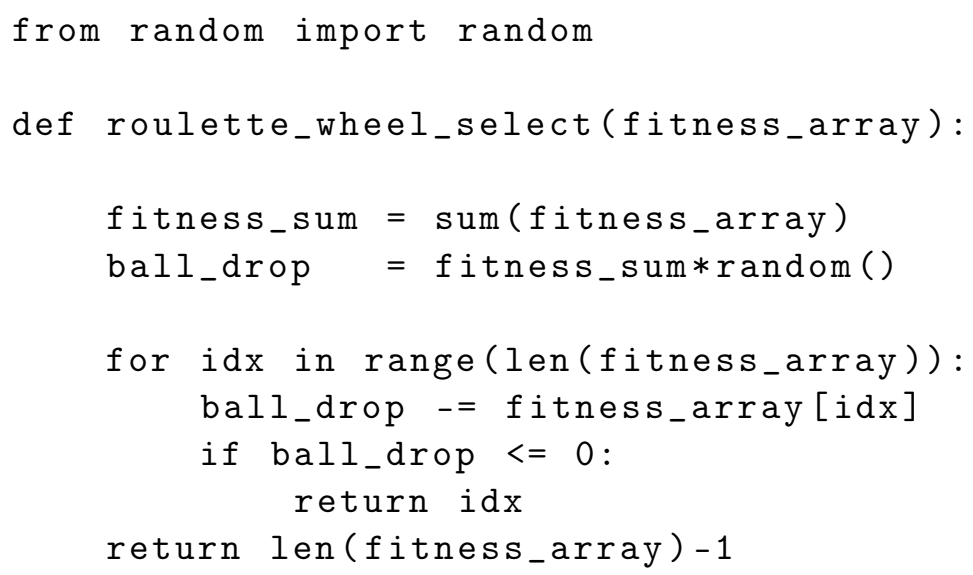

Figure 4: Roulette wheel selection Python implementation determining which individuals shall procreate to contribute children to the next generation. fitness_array has the same size as the population and its entries are the computed fitnesses of each individual. fitness_sum determines the highest-numbered pocket on the wheel and ball_drop is chosen at random between zero and fitness_sum. The return value of the function is the index of the pocket that the ball fell into. The last line's purpose is to ensure a return value of the function in case of rounding problems.

between zero through thirteen is drawn from a uniform distribution. This number serves as a the locus of fragmentation of both parental EMT parameter chromosomes. Second, these fragments are swapped around so that both children end up with fragments of both parents. Note that these fragments originate from locally optimized EMT parameters. The new combinations most likely do not correspond to minima of the fitness function, but this guess is probably more educated than in the previous generation. Third, this cut and exchange procedure is repeated with the chromosome that determines what parameters shall be locally optimized in the next call to md_tian. Fourth, each parent passes its geometry data of an AIMD trajectory to a child, together with the fraction of data points to use during the next parameter optimization. This scheme is repeated until the new population has the same size as the old population.

Now that the genetic makeup of the new generation is determined, the optimization could in principle be started. However, recombination, though a crucial part of the GA's global character, can only mix existing results. The exploratory part must be introduced by the mutation operator to possibly find better solutions in unexplored parts of search space. The mutation rate is a somewhat delicate measure, as too small values lead to only little exploratory activity, and too high values turn the GA into a random walk. An allele on the EMT parameter chromosome is mutated with a probability of $3 \%$. The mutation itself is carried out by applying a Gaussian 
blur with a $5 \%$ standard deviation of the mean. Here, the mutation of EMT parameters rather plays a minor role since they are changed by local optimization anyway with every new generation. More importantly, an individual might be assigned a new AIMD trajectory to use for training and also the fraction of AIMD geometries is subject to mutation. The latter mutation is also performed using Gaussian blur with a small standard deviation of the mean. Both mutations happen with a probability of 5\%. The binary chromosome that determines what EMT parameters are optimized by md_tian is mutated by applying the negation operator to each element with a probability of $3 \%$.

Extensive statistics about each generation are collected like average fitness, the fitness spread across a population, usage counts of AIMD trajectories and many more. Some of them are shown in section 4.1.2. The stopping criterion in this GA is a visible plateau of the maximum fitness over dozens of generations. One generation usually consists of 300 individuals, so that the maximum fitness is a reasonable measure for convergence.

\subsection{MDT2}

md_tian is a very useful program to simulate scattering phenomena of $\mathrm{H}$ atoms on gold. With some work towards generalization by removing hard-coded, gold-specific constants, it was possible to extend it to other fcc metals. Since this approach yielded very sensible results in great agreement with experiment, a substantial part of this work was to extend the program in various directions. As it turned out, the internal structure of md_tian did not allow for modular extensions to other MD PESs, propagation methods or even to the body-centered cubic (bcc) crystal system. Hence, I wrote a new MD program called 'MDT2' that incorporates some general concepts from md_tian, but with a completely new structure.

The design of MDT2 clearly focuses on modularity. It shall overcome all shortcomings of md_tian in terms of extensibility to new PESs, propagation algorithms and $\mathrm{I} / \mathrm{O}$ formats amongst others. Fortran modules are used in all source files and subroutines are designed to have as little side effects and interdependencies as possible. This allows for simple improvements and substitutions of parts of the program without having to worry about the functionality as a whole. MDT2 makes use of Fortran interfaces to automatically select the suitable subroutine based on the number or dimension of function arguments. Variable scopes are limited as much as possible to ease maintenance and object-oriented approaches are used where sensi- 
ble. The input file has a simple structure that consists of key-value pairs and was adapted from md_tian.

Several commands are mandatory for MDT2 to run that have to be provided by the user. Among them is a path to a PES parameter file. The user has to specify an interaction potential between all atom types present in the calculation and whether this type is the projectile or part of the surface. Then, the program internally builds an interaction matrix of rank $n$ where $n$ is the number of different atomic types. Later in the energy and force evaluation, the interaction potential of the two types can be simply looked up in this matrix.

MDT2 also needs to be provided with a file containing the geometry of the system. The layout was strongly inspired by the VASP POSCAR file with some minor modifications. The number of atoms for example together with the atomic symbol is required and checked against the specifications in the PES and general input file. For this purpose, there exist several sanity check subroutines. They check the layout of all files being read in separately and then verify that there are no conflicts or ambiguities between different files. Besides that, the program can work with binary geometry files created by MDT2 during a previous execution. This feature was adapted from md_tian and is especially useful when running a large number of MD trajectories. In this case, the initial surface geometry can be sampled from a pool of pre-equilibrated binary snapshots.

Maybe the biggest addition to md_tian is the implementation of Ring Polymer Molecular Dynamics (RPMD) (cf. 2.4.3). This method enables the user to look at tunneling and zero point energy effects which is impossible to do with classical MD. At the point of this writing, this is the first time that scattering simulations can be performed with the RPMD approach to quantum effects. The module includes all necessary subroutines for the propagation of the ring polymers. The unitary matrix to convert from Cartesian coordinates to normal modes and vice versa is built at runtime. It is used in the integration step of the equations of motion since normal mode propagation conserves the ring polymer phase space volume for arbitrary time steps. On the one hand, if the propagation was performed in Cartesian coordinates, it would eliminate the overhead of converting back and forth. But on the other hand, keeping the total energy conserved would require a very small time step due to rapid oscillations in the ring polymer. Especially in cases where the harmonic springs are very stiff, i.e., heavy atoms, high temperature or many beads, normal mode propagation is the only viable method. In the future, the subroutine could be replaced with a fast Fourier transform to test for a possible speed-up. Besides that, 
the RPMD module contains a number of functions to monitor the ring polymers during a trajectory. The radius of gyration, inter-bead distances and the quantum kinetic energy virial estimator provide information about attractive or repulsive interactions with the surroundings. For the extraction of features at the end of the trajectory, there are functions that project properties on the centroid mode to facilitate the analysis.

The main program is structured in a straightforward way: in any case, the system is first initialized as a singleton object of the 'universe' class. This is a software design pattern, that prevents multiple instances of the same class from being created. When the class is queried for the first time, all input files are read, verified, and the singleton is instantiated. In all subsequent calls to this class, no re-instantiation takes place, but instead, the existing singleton is returned. Next, the program proceeds through a couple of case statements. The geometry optimization branch has already been described in section 2.5.3 and information on the fitting routine was detailed in section 3.2. In MDT2, the fitting routine can be used in parallel via the OpenMP standard [119]. It is compatible with the Intel Math Kernel Library and can achieve considerable speed ups compared to the sequential version. One does not have to worry about any overhead in this case, because the trust region algorithm is much more demanding than any variable initialization or hard disk access. The only issue with this approach is load balancing. Several compiler options need to be tested on a system so that the program knows how many CPUs and threads are available and how to distribute the jobs evenly. In any case, many instances of MDT2 each running on one processor will necessarily be faster than one instance running on multiple processors.

As of this writing, the only remaining branch is the MD part whose core functionality consists of five to six steps, depending on the propagation scheme. Usually, a trajectory is simulated in the $N V E$ ensemble and the velocity Verlet algorithm is chosen as default for propagation. Then these iteration steps read:

(1) Update velocities by half a time step. If RPMD is not active, update positions by one time step.

(2) If RPMD is active, propagate ring polymer normal modes by one time step.

(3) Calculate energy of the system and update all forces. Only call subroutines which are indexed in the interaction matrix.

(4) Update velocities by half a time step. 
(5) Collect information about the state of the trajectory.

(6) If requested, write output to disk.

To equilibrate a system to a certain temperature using the $N V T$ ensemble, for example to prepare trajectory simulations or look for the disintegration temperature, MDT2 offers two thermostats. Details about them can be found in section 2.4.4. The Andersen thermostat is especially simple to use. One only has to define a collision frequency and the desired temperature in the input file and monitor the verbose output, which is described later in this paragraph. The Path Integral Langevin Equation (PILE) is recommended for thermostatting RPMD simulations, but can also be used for classical dynamics. It also only requires one input value apart for the requested temperature. This value then describes a friction coefficient of the ring polymer centroid mode, which coincides with the classical atomic motion if RPMD is not used. Both thermostats eventually lead to the correct Boltzmann phase space distribution whether RPMD is employed or not. PILE is in principle faster than the Andersen thermostat, but only if a good guess for the friction is available.

The program uses an optimized and extended version of the EMT PES from md_tian. There is a generic implementation that supports an arbitrary number of interacting atom types. Hence, it is possible to simulate atom scattering from an alloy, whereas in md_tian, one was limited to a surface consisting of one element. This approach unfortunately leads to computations of six-dimensional matrices in case of RPMD when the beads of index $b$ of atoms $i$ and $j$ being atom types $m$ and $n$ are interacting in the Cartesian coordinate $x$. To avoid calculating these very sparse matrices, MDT2 incorporates optimized versions of EMT. They are called in case only one or two atom types are present in the simulation cell since this is the common use case. The speed up was realized to a large extent by dimensionality reduction of the involved matrices. All mentioned module procedures are listed in the EMT Fortran interface so that the ideal subroutine can be determined at runtime.

Internally, the desired propagation algorithm, PES and output format are translated into IDs. This allows for clearly arranged blocks of possible options in the code. Moreover, if a new PES shall extend the functional range of MDT2, of course a new module, ID and character array for I/O functionality must be created. Then, a case statement must be added manually to the list of possible indices in the interaction matrix. But these few steps describe everything which is required to seamlessly integrate a new PES into MDT2.

Another big addition in terms of PESs in MDT2 is the Reactive Empirical Bond 
Order (REBO) potential (cf. Sec. 2.3.2). This module alone has half the source lines of code of the original md_tian program. In the first call to the module, the initialization subroutine solves several systems of linear equations to obtain spline coefficients using Intel LAPACK [95]. All spline functions combined, there are over 40,000 coefficients in REBO and reading them from the hard drive turned out to be slower than calculating them 'on-the-fly' in memory. To further reduce runtime, there is a strong focus on accessing multi-dimensional arrays in a coalesced fashion. The compiler and build flags also affect performance to a great deal, but this is true of course for the entire MDT2 program. However, the REBO potential is computationally by far the most demanding PES in MDT2. The energy and forces scale with $\mathcal{O}\left(n^{4}\right)$ and $\mathcal{O}\left(n^{5}\right)$ respectively, with $n$ being the number of atoms in the simulation cell. It is therefore advisable to use the least possible number of atoms which still capture the important dynamics.

It is also possible to use several PESs in a simulation. MDT2 can handle an arbitrary amount provided that all parameters are listed in the PES input file. Care must be taken with the system potential energy in this case. It is only informative if either all PESs are based on total energy or all of them describe interaction energies. The balancing of forces is straightforward since they are additive and only depend on the derivatives of the potential energy functions, but not their absolute values. One use case of this feature is the simulation of $\mathrm{H}$ atom scattering from graphene grown on a weakly interacting metal substrate. The REBO PES can be used for $\mathrm{C}-\mathrm{C}$ and $\mathrm{C}-\mathrm{H}$ interaction, EMT can describe the metal bonds and their interaction with the projectile and van der Waals forces modeled by a LJ potential attach the graphene sheet to the metal. An overview about the implemented PESs and methods of propagation is given in Tab.2. Centroid Molecular Dynamics (CMD) has been extensively discussed elsewhere [120, 121] and was not used in this work. Nevertheless, it is important to note that the modular program design allows for an addition of this scheme if it is needed for future simulations. Since RPMD and CMD are both approximations to Matsubara dynamics [122, 123], one could examine the possibility of approximating Matsubara MD via the planetary model [124] as well. It is of course computationally very expensive due to the explicit treatment of phases and one would need to change to complex floating point numbers. It should be feasible, however, for anyone with skills in software engineering.

MDT2 supports a variety of output formats. An exemplary usage of different formats for different tasks may look like this: the equilibrium geometry of a system is used as the initial configuration and then one performs an NVT ensemble calculation 
Table 2: Implemented $(\boldsymbol{})$ propagation schemes and PESs in MDT2. Possible further development $(\boldsymbol{x})$ includes the addition of potentials to describe more atomic species, and propagation algorithms to approximate quantum dynamics differently.

\begin{tabular}{ccccccc} 
& \multicolumn{5}{c}{ Potential energy surface } \\
& & Harmonic & Lennard- & \\
& & Oscillator & Jones & EMT & REBO & Morse \\
Method of & RPMD & $\checkmark$ & $\checkmark$ & $\checkmark$ & $\checkmark$ & $\checkmark$ \\
propagation & CMD & $\boldsymbol{x}$ & $\boldsymbol{x}$ & $\boldsymbol{x}$ & $\boldsymbol{x}$ & $\boldsymbol{x}$ \\
& (Matsubara) & $\boldsymbol{x}$ & $\boldsymbol{x}$ & $\boldsymbol{x}$ & $\boldsymbol{x}$ & $\boldsymbol{x}$ \\
\hline
\end{tabular}

to simulate the system at room temperature. At the end of the calculation, MDT2 can output the atomic positions and velocities. This file can immediately be used as the input for an $N V E$ ensemble to generate surface snapshots for MD trajectories. These snapshots are written to disk as binary files so that they can be read quickly at the beginning of a scattering simulation. The trajectory data can be written to disk in several ways. Most often, one simulates ten thousands of scattering events. In this case, minimum output is preferable, which contains some initial and final conditions like duration, projectile kinetic energy and scattering angles. For a more detailed analysis, a verbose output can be used that includes surface and projectile kinetic energy, potential energy, total force, and in case of RPMD, radius of gyration and quantum kinetic energy at every $n^{\text {th }}$ time step. To gain more insight about how the trajectory progresses, MDT2 supports the $x y z$-format which is an ASCII file displaying mainly the atomic coordinates at regularly spaced intervals during a trajectory. This file can be read with any molecular modeling and visualization computer program. Just like the verbose output, the interval $n$ can be specified in the input file to MDT2 to balance information content and disk usage. 


\section{$4 \mathrm{H}$ on fcc Metals}

To accurately predict interactions at surfaces, it is mandatory to know the details of the surface structure. Early experimental results using temperature programmed desorption [125] could already reveal coarse features. But the real advent of surface chemistry started when techniques like scanning tunneling microscopy [126], low-energy electron diffraction $[127,128]$ and high resolution electron energy loss spectroscopy [129, 130] entered the field. They finally allowed to accurately determine adsorbate geometries, surface steps and terraces and helped with elucidating mechanisms in heterogeneous catalysis.

On the theory side, the advances came alongside the experimental successes. The London-Eyring-Polanyi-Sato (LEPS) Potential Energy Surface (PES) was the first of its kind to model chemical reactions. It was conceived of in 1929 by London [131] and it took 45 additional years to be successfully applied to gas-surface interactions. This PES is limited to six dimensions, but can describe $\mathrm{H}_{2}$ on a $\mathrm{W}(001)$ surface. It was used for $\mathrm{H}_{2}$ scattering simulations [132] and $\mathrm{H}_{2}$ recombination on the tungsten surface [133]. The basis is a parametrized Coulomb and exchange interaction for which Morse parameters are typically used. The Sato parameters have to be optimized to model barrier heights and their locations. The model was later extended to include the periodicity of the surface in the periodic LEPS PES called PLEPS. It could be used in many dissociative adsorption reactions like $\mathrm{H}_{2}$ on $\mathrm{W}(110)$ [132], $\mathrm{Fe}(001)$ [134], $\mathrm{Ni}(100)$ [135] and $\mathrm{Cu}(111)$ [136], as well as for the description other diatomics like $\mathrm{N}_{2}$ [137] and $\mathrm{O}_{2}$ [138]. A further refinement of this theory called flexible PLEPS or FPLEPS was published in 2009 by Martin-Gondre and coworkers [139]. They noticed that PLEPS fails to model $\mathrm{N}_{2}$ dissociation on $\mathrm{W}(100)$ correctly and devised improvements to describe short and medium range interaction energies. An angular dependence of surface corrugation was also added and results were published for $\mathrm{N}_{2}$ on $\mathrm{W}(100,110)$ [140] and on non-metallic systems [141, 142] among others.

In another approach, Daw and Baskes came up with the Embedded Atom Method (EAM) $[143,144]$. It can be used to model ground state properties of metals and also gas-surface interactions. Its basis is the calculation of an embedding energy of atoms in the homogeneous electron gas. The electron density that an atom feels can be calculated from density tail contributions of the surrounding atoms. The model includes an explicit short-range Coulomb term which gets added to the embedding energy. There are numerous examples of a successful application of this theory for 
example to $\mathrm{H}_{2}$ on $\mathrm{Ni}(100)$ [145], $\mathrm{Cu}(100)$ [146, 147], $\mathrm{Ni}(410)$ and $\mathrm{Cu}(410)$ [148] and Pt(111) [149]. Based on the approach, Nørskov and coworkers [56, 57] developed the Effective Medium Theory (EMT) that was able to predict bonding properties of $\mathrm{H}$ atoms on metal surfaces [150]. EMT is used to model all hydrogen-metal interactions in this work and is described in detail in section 2.3.1. This brief historical overview about theory was mainly inspired by Ref. [23] in which much more information on this topic can be found.

Nowadays, thirty years from Nørskov's publication, high computational power is accessible to anyone. This situation is of course dramatically different and allows for a greater variety of high level $a b$ initio calculations. And though the advancement is so remarkable, even the simulation of a single microsecond is by far out of reach when Density Functional Theory (DFT) accuracy is required. On this timescale pre-calculated force fields or PESs are indispensable. In this section I present how an approach using the best of two worlds, namely the accuracy of DFT coupled to the speed of a PES, builds on Nørskov's work to model $\mathrm{H}$ atom scattering from a range of face-centered cubic (fcc) metal surfaces.

\subsection{EMT PES}

\subsubsection{Data Preparation}

The following paragraphs will describe the protocol that was used to fit EMT PESs to describe a hydrogen atom at various fcc metal surfaces. In the first part, I will explain in detail how the DFT reference data was produced. One starts with optimizing the bulk lattice constant and uses it to construct a relaxed slab. Then an $\mathrm{H}$ atom is placed a several high symmetry sites above and inside the surface to systematically scan the energy landscape. The calculation of a few ab initio Molecular Dynamics (AIMD) trajectory completes the data acquisition stage. The second part will be about the training and validation process of the PES. The generation of PESs that describe $\mathrm{H}$ at aluminum, nickel, copper, rhodium, palladium, silver, platinum and gold all follow the same protocol. Most of the time, the Potential Energy Curves (PECs) look very similar across the range of metals. Hence, the parametrization process will be documented by the most illustrative calculations which are not necessarily all with the same metal. The Perdew, Burke, and Ernzerhof (PBE) functional [39] was used for all DFT calculations concerning $\mathrm{H}$ on metals if not stated otherwise. The PW91 [151] or revPBE [152] functional are alternatives to use for these calculations. In summary, they are all well-established General- 


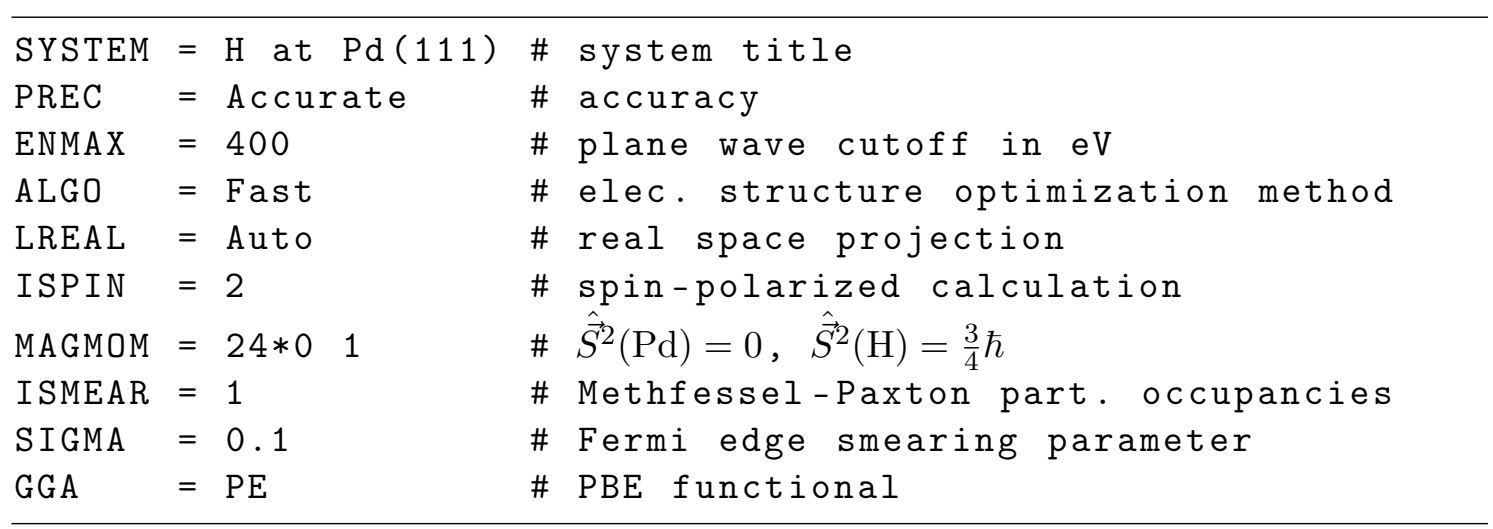

Figure 5: Exemplary INCAR file for the total energy calculation of a hydrogen atom in the vicinity of a palladium surface.

ized Gradient Approximation (GGA) functionals and give similar results for lattice constants, surface energies and chemisorption well depths. Throughout all different stages, the $k$-point mesh is generated by the Monkhorst-Pack scheme [153] using four subdivisions along each reciprocal lattice vector. Methfessel-Paxton Fermi edge smearing [154] with a width of $0.1 \mathrm{eV}$ is used for all metals. The plane wave energy cutoff is set to $400 \mathrm{eV}$. At the end of this section, I will show some statistics of applying the Skycruiser package to this global optimization problem of fitting the PES to the DFT reference data.

The Bulk The first step towards the construction of an accurate full-dimensional PES is to find the bulk lattice constant of the metal of interest. Although it might seem immediately clear, even trivial, such a simple calculation already yields one EMT parameter, called $s_{0}$. One starts by obtaining information about the recommended plane wave cutoff of this system. This number can be found in each POTCAR files of the involved atomic types and the highest one is used for all calculations of this system. It is important to think about which elements are going to be included in future calculations right from the start. Else, one might optimize structures for a certain cutoff and later realize that a second element in the simulation requires a higher one. This might lead to inconsistencies in equilibrium geometries for example. Fig. 5 shows an exemplary input file for computing the energy of an $\mathrm{H}$ atom near a metal surface.

The POSCAR file for bulk Pd is shown in Fig. 6. It contains a single atom and the described primitive cell is a parallelepiped of edge length $\sqrt{2} a$ inscribed into the $\mathrm{Pd}$ unit cell, where $a$ is the lattice parameter. Since this is a very fast calculation, the 


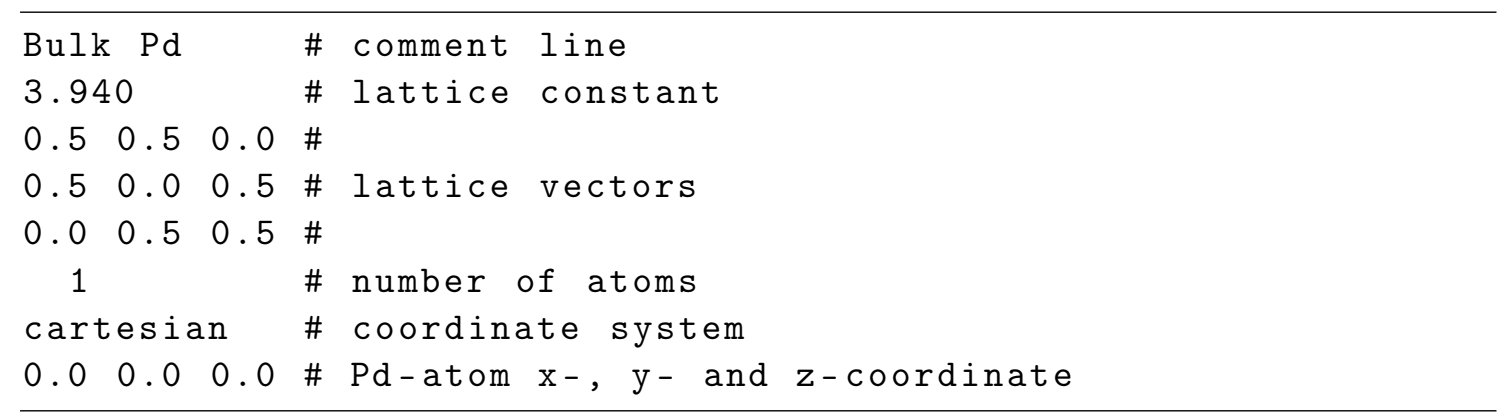

Figure 6: POSCAR file for the calculation of the Pd bulk energy.

Table 3: Calculated lattice parameters $a_{0}$ in $\AA$ of all studied metals and comparison to literature values.

\begin{tabular}{rcccccccc}
\hline & $\mathrm{Al}$ & $\mathrm{Ni}$ & $\mathrm{Cu}$ & $\mathrm{Rh}$ & $\mathrm{Pd}$ & $\mathrm{Ag}$ & $\mathrm{Pt}$ & $\mathrm{Au}$ \\
\hline DFT & 4.05 & 3.50 & 3.64 & 3.80 & 3.94 & 4.16 & 3.96 & 4.03 \\
Ref.[156] & 4.05 & 3.52 & 3.62 & 3.80 & 3.89 & 4.09 & 3.92 & 4.08 \\
\hline
\end{tabular}

equilibrium lattice constant $a_{0}$ can be found by simply scanning a range of $\pm 0.1 \AA$ around the literature value.

Tab. 3 shows the PBE DFT equilibrium lattice constant and the literature value of all studied metals. We can see the general trend that GGA DFT tends to overestimate the lattice parameter. This is due to the larger dependence on the density gradient $[39,155]$. In consequence phonon frequencies will be softened, but they play a minor role in scattering dynamics anyway. However, it is true that GGA functionals better reproduce atomization energies than those based on the Local Density Approximation (LDA).

The Slab With the lattice constants at hand, a (111)-surface can be constructed. It is displayed in Fig. 7 together with the standard fcc unit cell. Because the Vienna ab initio Simulation Package (VASP) uses plane waves, the system extends infinitely in all dimensions. This is convenient for bulk calculations, but the periodicity normal to the surface (called $z$-direction in this work) is uncalled-for, sometimes even troublesome, when simulating slabs. The best way to deal with it is to introduce a vacuum region above the slab. In the VASP picture, there is now a periodic succession of metal slab and vacuum in the $z$-direction. After testing vacuum regions up to $15 \AA$, I found that $6 \AA$ suffices to prevent any spurious interaction between the stacked slabs. Also setting the $k$-points in $z$-direction to one greatly helps reducing 


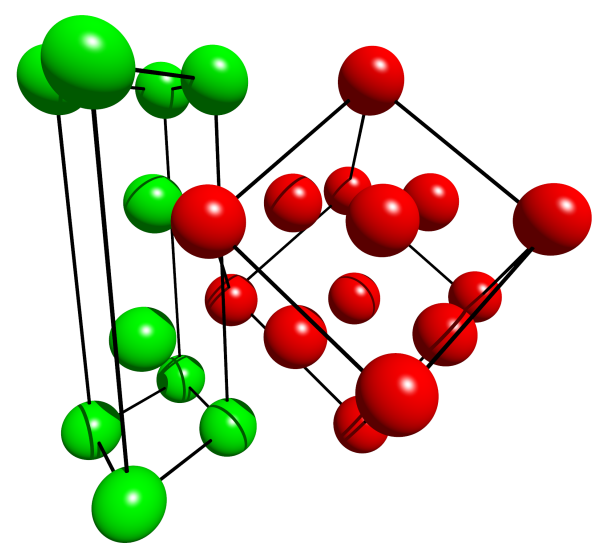

Figure 7: Red atoms indicate the standard fcc unit cell. It is positioned on one corner to highlight the geometric origin of the atoms in the primitive (111) cell shown in green.

any dispersive interaction. In general, one wants to keep the simulation cell as small as possible. Regardless of any void VASP evenly distributes the plane waves in the simulation cell. They do not have any amplitudes in the vacuum region of course, but they nevertheless prolong the calculation.

Since $6 \AA$ is enough to prevent dispersive slab interactions in the $z$-direction, I settled on a vacuum of $13 \AA$ for the slab relaxation and subsequent calculations involving hydrogen. The argument is that the metal electron cloud extends further into the vacuum than the single electron of the hydrogen atom. Therefore, if there is no periodic interaction in $z$-direction, an $\mathrm{H}$ atom $6 \AA$ above the metal surface is also interaction-free. However, there is no way to get rid of periodic $z$-interaction. Hence, with the same reasoning, if an $\mathrm{H}$ atom is sandwiched between two metal slabs each at a $6 \AA$ distance, it must also be interaction-free. As a last step, I increase the vacuum region by $1 \AA$ just to be on the safe side. The advantage is that the slab dimension can be kept fixed during relaxation and all subsequent steps.

Regardless of the choice of lattice vectors, the energy changes during the transition from a bulk system to a slab. Atoms in the surface layer are missing neighboring atoms that have been replaced by the vacuum region. In consequence, the distance between the surface layers changes due to the new geometry. Most fcc metals and surface cuts exhibit an inward relaxation in both theory and experiment. This can be either explained by a reduction in electronic corrugation which is connected to the kinetic energy of the electrons, or by the bond order-bond length correlation [157]. The surface energy $E_{\text {surf }}$ can be calculated by

$$
E_{\text {surf }}=\frac{1}{2}\left(E_{\text {slab }}^{\mathrm{N}}-N E_{\text {bulk }}\right)
$$

where $E_{\text {slab }}^{\mathrm{N}}$ is the total energy of the slab consisting of $N$ atoms and $N$ times the 
Figure 8: Schematic display of the ten sampled surface sites. Red spheres mark atoms in the surface layer. Green symbolizes the first subsurface layer and the blue refers to an atom in the second subsurface layer. More information about the lateral $\mathrm{H}$ atom positions indicated by the numbers can be found in Tab. 4 .

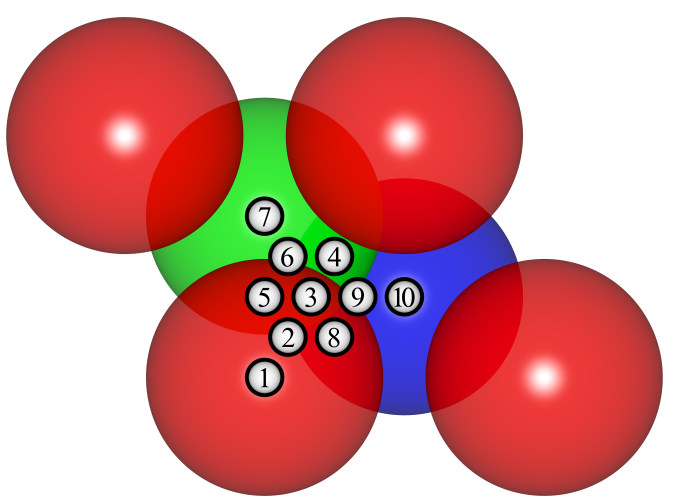

energy of an atom in the bulk $E_{\text {bulk }}$. The factor of one half accounts for the fact that a slab has two surfaces. Just like the lattice constant, the surface energy must be compared to literature values to ensure that the correct minimum has been found.

The purpose of the slab calculation is to optimize the geometry of the slab starting from the equilibrium bulk geometry. Due to missing neighbor atoms above and below the slab, the surface layers will adapt their inter-layer distances to the new electronic situation. I decided to use a six-layered slab in all cases for two reasons. First, the surface energy (see Eq. 4.1) should be as close as possible to the converged PBE value. Several tests have shown that this quantity can be reproduced to $2 \%$ accuracy using six layers. Second, using six layers, the $\mathrm{H}$ atom has a very low probability to traverse the slab during the AIMD trajectory. Once the $\mathrm{H}$ atom is launched towards the slab, its path becomes unpredictable. And since the purpose is to visit as many configurations as possible that would appear in a real system, traversing $\mathrm{H}$ atom give a description of an unrealistic situation.

The DFT Grid With a relaxed surface slab at hand, the first DFT reference data points can be calculated that will later be used for fitting the PES. Following earlier efforts in this group [158], the hydrogen atom is placed above and inside the surface at high symmetry sites. Fig. 8 provides a top view of the $\mathrm{H}$ atom positions over a (111) surface and Tab. 4 lists the coordinates in terms of the lattice constant $a_{0}$. In this grid of DFT single point energies, the slab is kept at its equilibrium geometry. The vertical $\mathrm{H}$ atom positions range from $6 \AA$ above the slab to the bottom atomic layer in steps of $0.2 \AA$. This way many configurations are created in a grid-like fashion which can all be encountered in a Molecular Dynamics (MD) scattering simulation. All of the approximately 800 single point energies that make up the DFT grid are used for fitting the PES. 


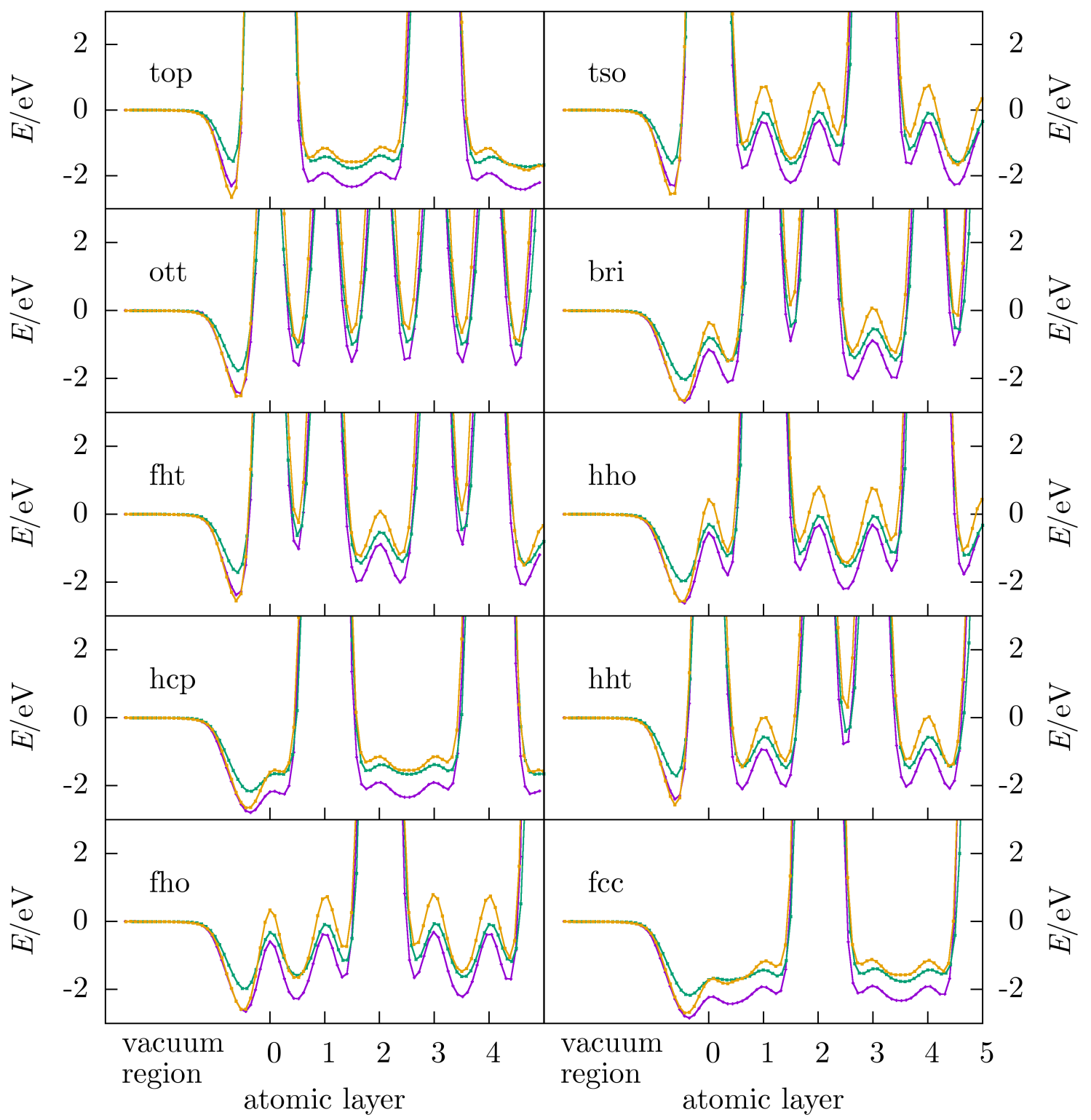

$$
-\mathrm{Ag}-\mathrm{Pd}-\mathrm{Pt}
$$

Figure 9: Energy of an $\mathrm{H}$ atom at ten different sites above and inside the metal slab calculated by DFT. The $z$-direction is normalized to the inter-layer distance of the respective crystal lattice. Three metals were chosen to illustrate the range of interaction energies that one can expect in these types of systems. See the text for a detailed discussion. 
Table 4: List of the ten sampled surface sites. The coordinates are expressed in terms of the lattice constant since they are the same for all fcc metal (111) surfaces. Abbreviations are adapted from Refs. [158] and [159].

\begin{tabular}{rcccl}
\hline site & $x / a_{0}$ & $y / a_{0}$ & abbreviation & description \\
\hline 1 & 0.0 & 0.0 & top & on top of a surface layer atom \\
2 & $\frac{1}{6 \sqrt{2}}$ & 0.0 & tso & between top and ott \\
3 & $\frac{1}{3 \sqrt{2}}$ & 0.0 & ott & $\frac{1}{3}$ the distance between top sites in $x$-direction \\
4 & $\frac{1}{2 \sqrt{2}}$ & 0.0 & bri & between top sites in $x$-direction \\
5 & $\frac{1}{4 \sqrt{2}}$ & $\frac{1}{4 \sqrt{6}}$ & fht & between top and hcp \\
6 & $\frac{5}{12 \sqrt{2}}$ & $\frac{1}{4 \sqrt{6}}$ & hho & between hcp and ott \\
7 & $\frac{1}{2 \sqrt{2}}$ & $\frac{1}{2 \sqrt{6}}$ & hcp & hexagonal cubic package hollow \\
8 & $\frac{1}{4 \sqrt{2}}$ & $-\frac{1}{4 \sqrt{6}}$ & hht & between top and fcc \\
9 & $\frac{5}{12 \sqrt{2}}$ & $-\frac{1}{4 \sqrt{6}}$ & fho & between fcc and ott \\
10 & $\frac{1}{2 \sqrt{2}}$ & $-\frac{1}{2 \sqrt{6}}$ & fcc & face-centered cubic hollow \\
\hline
\end{tabular}

The energies of an $\mathrm{H}$ atom interacting with some of the studied metals are presented in Fig. 9. We see that the curves in each panel share similar characteristics. This is to be expected since the potential energy in close proximity of an atomic core is necessarily high. Between the atomic layers and in high symmetry subsurface sites, the energy is lower in any case. This knowledge already explains the extrema in Fig. 9. Concerning absolute values of the interaction energy, palladium is most attractive throughout all surface sites. This is explained by the fact that it has the highest absorptive capacity for hydrogen of all elements. Platinum has about the same chemisorption energy above the surface, but is more repulsive in the subsurface region. This diverse energy landscape makes the $\mathrm{Pt}-\mathrm{H}$ interaction especially difficult to parametrize using the EMT PES. The shape of the Ag and Pd PECs look fairly similar. In numbers though, a silver surface is for the most parts around $500 \mathrm{meV}$ less attractive to an $\mathrm{H}$ atom than $\mathrm{Pd}$. For this reason, the $\mathrm{H}$ on $\mathrm{Ag}$ curve is most of the time located somewhere between Pd and Pt.

The AIMD Trajectories The next step is to generate slab geometries at a finite temperature. Room temperature was chosen in most cases because the experiments to which the scattering simulations can be compared also take place at room temperature. VASP implements several thermostats that can be used for thermalization, 
or, since the minimum energy geometry is known, the method of equipartition (section 2.4.4) can also be used. The thermalized geometries are later used as initial configurations for the AIMD trajectories. Several snapshots of the surface need to be available for the trajectories since a single configuration would not capture the Boltzmann phase-space average well.

To prepare several surface geometries at $300 \mathrm{~K}$, I start with the minimum energy configuration and draw the initial velocities from a Maxwell-Boltzmann distribution at twice the desired temperature. An $N V E$ ensemble is used and the temperature is monitored over $1 \mathrm{ps}$. By then, the average temperature has dropped to $300 \mathrm{~K}$ because the method of equipartition works well in this low temperature regime for all described metals. From this point on, snapshots of the surface are saved every $100 \mathrm{fs}$ until a dozen of them are available. The time step is set to $0.25 \mathrm{fs}$ because all motions are fairly slow when no $\mathrm{H}$ atom is present. The energies encountered during the AIMD trajectories play an important role in the fitting process because they introduce slab geometries at finite temperature. The DFT grid is a solid backbone to cover important, high-symmetry sites above and inside the surface. But since the surface is fixed, it contains no information about the force constant of the metallic bonds. Forces are of course a crucial aspect of a full-dimensional PES.

However, I decided against a direct inclusion of forces into training and validation data for the following reasons. First, the objective function would be difficult to define. One could of course have two error indicators at the end, one for energies and one for forces. But that would only delay the decision whether precise forces or precise energies are more important as a characteristic of a good fit. Second, the data set would grow rapidly with the system size. If $N$ is the number of atoms in the simulation cell, there is only one energy associated with it, but $3 N$ forces. Third, if configuration space is sampled appropriately, there is no need to take into account any derivatives. The sampled points already form a dense enough structure that makes forces obsolete and even detrimental. Nevertheless, the accuracy of forces can be checked indirectly by comparing the disintegration temperature to literature values.

About a dozen AIMD trajectories at a certain temperature suffice to capture thermal contributions to the interaction energy. The setup used in the parametrization of metal-H PESs is the same in all cases. The $\mathrm{H}$ atom is placed initially $6 \AA$ above a six-layered slab at room temperature. The bottommost layer must be fixed during the trajectory. On the one hand, this prevents the slab from floating around in the simulation cell and on the other hand it is far enough away from the $\mathrm{H}$ atom impact 


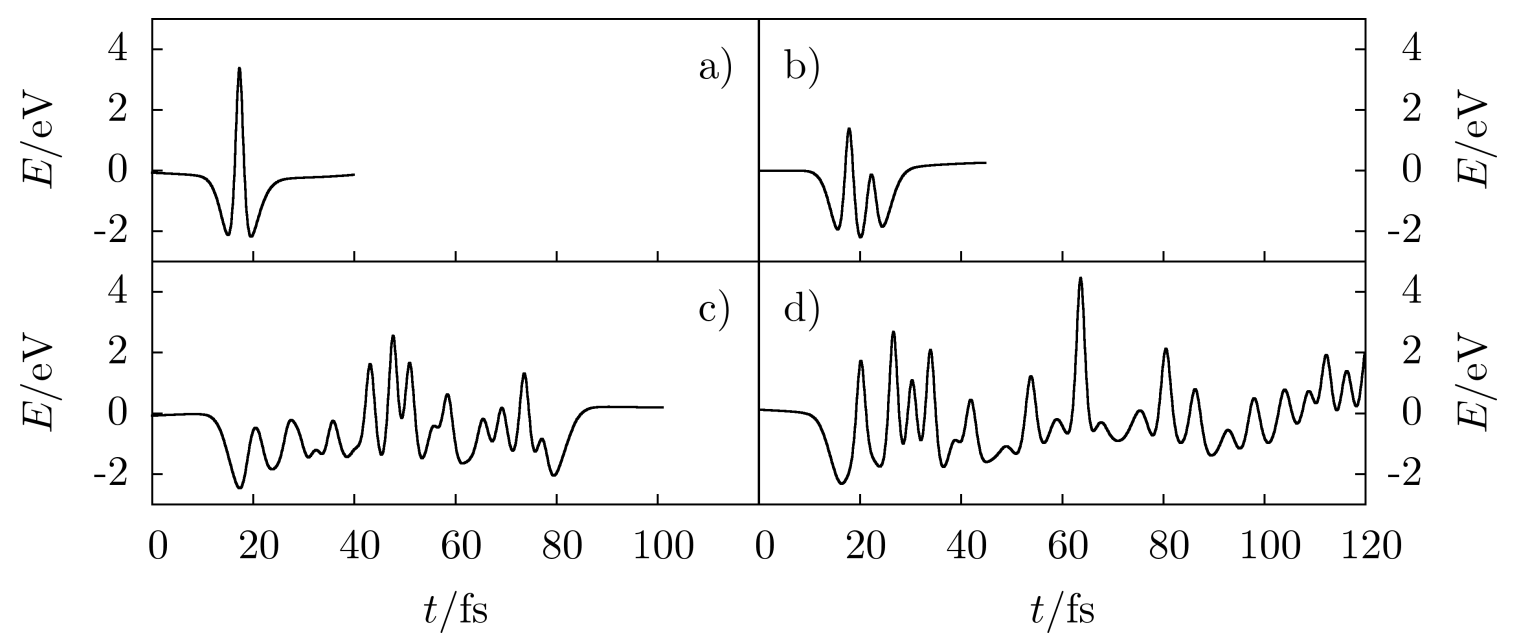

Figure 10: Potential energy of AIMD trajectories simulating the collision of an $\mathrm{H}$ atom with a $\mathrm{Cu}(111)$ surface. Panels a) and b) show a single- and double-bounce collision, respectively. Panel c) displays a trajectory in which the $\mathrm{H}$ atom is thrown back and forth between atoms of the top and the first subsurface layer before escaping into the vacuum. Panel d) presents a scenario in which the $\mathrm{H}$ atom stays subsurface for the entire duration of the simulation.

site that it does not influence the trajectory run. The incidence energy is set to $5 \mathrm{eV}$ to probe the energetic landscape well above the experimental conditions. The azimuthal angle can be chosen randomly, but here I use the [11 $\overline{2}]$-direction on the surface. This increases the probability of $\mathrm{H}$ penetrating into the surface because from this angle, the subsurface layers look most accessible. We actually want some $\mathrm{H}$ atoms to stay inside the surface until the end of the simulation. This produces a lot of data points in a fairly short amount of time because spin polarization does not have to be taken into account close to or inside the surface. The polar angle is set to $30^{\circ}$, the time step is $0.1 \mathrm{fs}$ and the run is aborted either after $120 \mathrm{fs}$ or when the projectile reaches its initial height after scattering.

The PECs from four AIMD trajectories simulating an $\mathrm{H}$ atom colliding with a $\mathrm{Cu}(111)$ surface is shown in Fig. 10. Panels a) to c) show curves describing a scattering event in which the $\mathrm{H}$ atom experiences a various number of collisions with the surface. In panel d), the incident particle is absorbed into the surface and stays inside the slab for the whole $120 \mathrm{fs}$ of simulated time. Single-, double- and multi-bounce trajectories were observed in all sets of AIMD calculations, regardless of the H-metal system. 


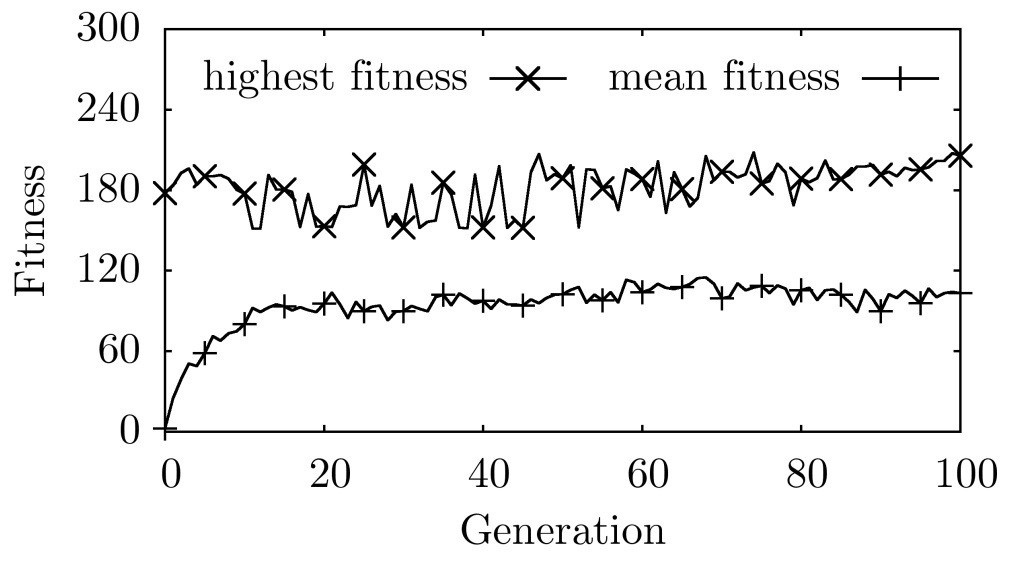

Figure 11: Development of the mean and highest fitness values during the optimization of the H-rhodium EMT PES. The unit of fitness is arbitrary, but completely determined by Eqns. 3.1 and 3.3. The average fitness improves a lot during the first 20 generations while the highest fitness shows a slow but steady increase.

\subsubsection{Fit}

After accumulating the data, it is passed to the Skycruiser wrapper script detailed in section 3.3. 60,000 individual fits in total are performed for each H-metal system. One generation usually consists of 300 individuals, so that the population has enough time to improve over the course of generations. The evolution of fitness can be monitored in real time while the algorithm is running and can be stopped when it reaches a plateau. Convergence has always been achieved within 200 generations. The evolution of the fitness is exemplarily shown in Fig. 11 for the H-Rh PES fit. The entire calculation takes approximately one week on an AMD Opteron 6272 server with 64 cores. It is interesting to see that the average fitness levels off after 20 generations and even in the first generation, at least one individual can already compete with the best ones of subsequent generations. This shows that simply guessing the initial PES parameters and applying a gradient descend minimization can already yield acceptable results for this system. Nevertheless, the robustness of this approach comes into play later on after dozens of generations have contributed to the parameter optimization. The oscillations in the highest fitness value are clearly smaller in generations 50 and higher, despite a complete replacement of all creatures from one generation to the next. Note that strategies like Elitism where the best individuals live over several generations would have prevented the highest fitness from ever decreasing, but it would not improve the overall Genetic Algorithm (GA) strategy and might lower diversity.

I used a second criterion for convergence besides leveling off of the average fitness in a population. In the end, the purpose is to perform accurate MD simulations on a full-dimensional PES. Therefore, it is important to check the dynamic aspects of the PES in MD simulations of scattering events. Using $\mathrm{H}$ on $\operatorname{Pd}(111)$ as an example, I ran 10,000 trajectories on each of the 60 highest ranked PESs to see how 
much variance is in the scattering statistics arising from different fits. The $\operatorname{Pd}(111)$ surface was at $300 \mathrm{~K}, \mathrm{H}$ atom incidence energy was $3.33 \mathrm{eV}$, the polar angle $\theta_{\mathrm{i}}$ was set to $45^{\circ}$ and azimuth $\varphi_{\mathrm{i}}$ was along the [101] -direction. I used the Local Density Friction Approximation (LDFA) (cf. section 2.4.2) to simulate kinetic energy loss due to electron-hole pair excitation. The simulations showed a sticking probability of $(61.8 \pm 0.8) \%$, an average kinetic energy loss of $(1.95 \pm 0.01) \mathrm{eV}$ and a most probable final kinetic energy of the projectile of $(2.79 \pm 0.05) \mathrm{eV}$ across all 60 PESs. In fact, half a percentage point of the observed sticking probability standard deviation can be explained purely by statistical noise. The same approach was used for all other H-metal PES parametrizations and consistent results were obtained for all of them. Though this observation makes the decision on the final PES for an H-metal system arbitrary, the results from dynamical simulations will not differ regardless of which fit is selected as the final one.

Another question that I want to answer with the GA approach is to what extend AIMD data contributes to the success of a fit. In principle, the total neglect of nonequilibrium data should lead to unphysical behavior, because there is no information about forces between metal atoms. On the other hand, including too many configurations at finite temperature should also deteriorate the quality of the fit. Since the data stems from a single AIMD trajectory, the redundancies would introduce clusters in configuration space increasing the Root Mean Square Error (RMSE) of the validation data set. In consequence there must be a sweet spot somewhere in between, which, given enough time to evolve, the GA should be able to localize. Important to note here is that I am not referring to the fraction of non-equilibrium configurations in the training data set. It is about the fraction of the available AIMD configurations, i.e., I want to include every $n^{\text {th }}$ point of the selected trajectory where $n$ is the quantity to optimize. In the GA approach, $n$ is optimized automatically since individuals with lower fitness are less likely to pass on their genetic information to the next generation. Hence, creatures with a disadvantageous allele in this regard will fade away over time.

The result is shown in Fig. 12 for six different metals. In the first generation the fraction of AIMD configurations is uniformly distributed among all individuals. Al, $\mathrm{Ag}$ and $\mathrm{Rh}$ show a very low final percentage of below 25\%. Cu converges between 10 to $35 \%$ and Ni between 25 and $45 \%$. The result is not so clear for Au. Though the contribution decreases over time which is the same behavior seen in all other systems, there still remains a fairly large spread after 100 generations mostly in the bottom half of the heat map. In the cases of $\mathrm{Cu}, \mathrm{Rh}$ and partly also of $\mathrm{Ag}$, 


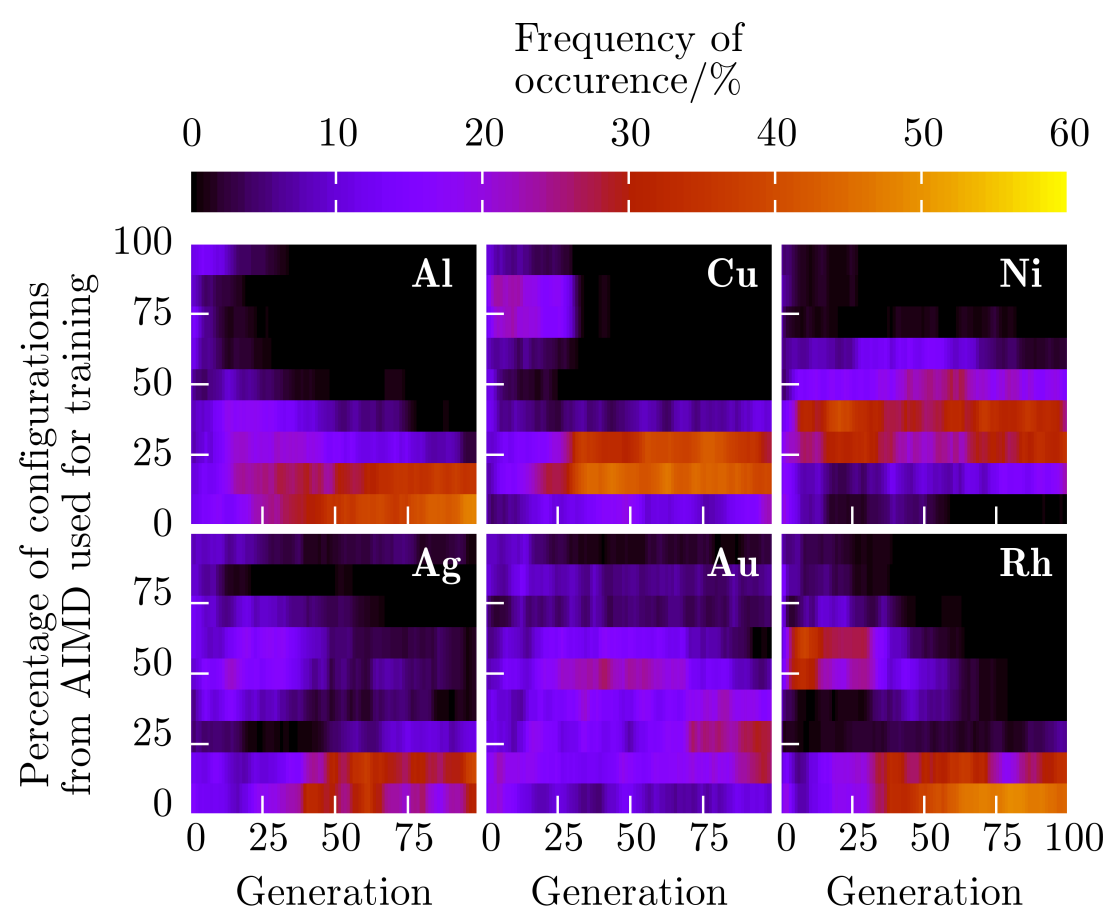

Figure 12: Heat map describing the evolution of AIMD contribution to the training data set during fitting. A clear development can be seen for most systems where the GA develops in favor of a small fraction of the available configuration/energy pairs from AIMD.

there are noticeable contributions well above $50 \%$ in the first 25 to 40 generations. Then at some point they vanish and do not reappear until the end of the run. This is another indication of convergence when there is a steady state between roulette wheel selection and mutation since lost traits can in principle be rediscovered by mutation.

I want to point out that the behavior in these complex systems is reproducible as long as hyper-parameters like mutation rate, objective function and selection operator stay the same. The percentage of configurations used from AIMD is also independent of the type of trajectory which is most common among the individuals. It does not seem to have any influence whether the non-equilibrium data is used from a trajectory in which the $\mathrm{H}$ atom spends most of its time diffusing through the slab or whether it describes a single bounce scattering event. This is understandable since the DFT grid already contains divers $\mathrm{H}$ atom positions above and inside the slab. If the main purpose of the non-equilibrium data is to enable tuning of the metal parameters, the type of $\mathrm{H}$ atom bounce event only plays a minor role. That is in fact good news for any future projects in this direction, especially in cases where only little computational power is available. One or two AIMD trajectories, 
which are definitely the computationally most intensive parts of the parametrization process, suffice to introduce enough non-equilibrium configurations into the training data set. In other cases, this knowledge is useful when one needs to decide what kind of trajectory to include in the input data set.

Now that the type of trajectory is identified as insignificant, we can compare the results of the GA with previous work done in this group. S. M. Janke describes in her dissertation [80] that she used 200 points from a non-penetrating doublebounce AIMD trajectory as input data at finite temperature. This is precisely the kind of trajectory that is shown in panel b) of Fig. 10. This simulation ends after $45 \mathrm{fs}$, and since we use both the same time step and stop criterion I assume her trajectory consists of around 450 configurations. This yields a utilized fraction of approximately $44 \%$ which is in reasonable agreement with the GA for the $\mathrm{H}-\mathrm{Au}$ PES.

Fit to DFT Grid The DFT grid serves as the backbone of the fit consisting of $\mathrm{H}$ atom positions above and inside the slab at selected high symmetry sites. Since all systems are (111)-surfaces of fcc metals, presenting results for all symmetry sites of every metal would be redundant. I therefore choose to show the quality of the fits with the help of the same metals as shown in Fig. 9. They cover the whole range of encountered adsorption energies and magnitudes of subsurface corrugation. Furthermore, these metals illustrate the drawbacks and opportunities of EMT. The number of panels was also reduced to only show the comparison between DFT and the fitted EMT potentials for the top-, tso-, ott- and bridge site. This eliminates further repetitions, because to a good approximation, the remaining six surface sites can be obtained by shifting the displayed PECs along the $x$-axis (top $\leftrightarrow$ hcp $\leftrightarrow$ fcc, tso $\leftrightarrow$ hho $\leftrightarrow$ fho, bri $\leftrightarrow$ hht $\leftrightarrow$ fht). At the end this paragraph will demonstrate for which metals EMT is a good choice as a full-dimensional PES, which are problematic and what are the traits already foreshadowing difficulties directly after the calculation of the DFT grid.

The $\operatorname{Ag}(111)$ surface is an example of a suitable system for the description with EMT (see Fig. 13). In comparison with other metals, the adsorption well is rather shallow and at the top-site it is very similar to the subsurface energetic situation. The same is true for the tso-site where the energy landscape below the surface can be well reproduced by EMT. The periodicity of the ott-site is captured remarkably. Merely at the bridge site some deviation is visible from the DFT reference points. The chemisorption well is fitted slightly too shallow and rather reflects the energies of 


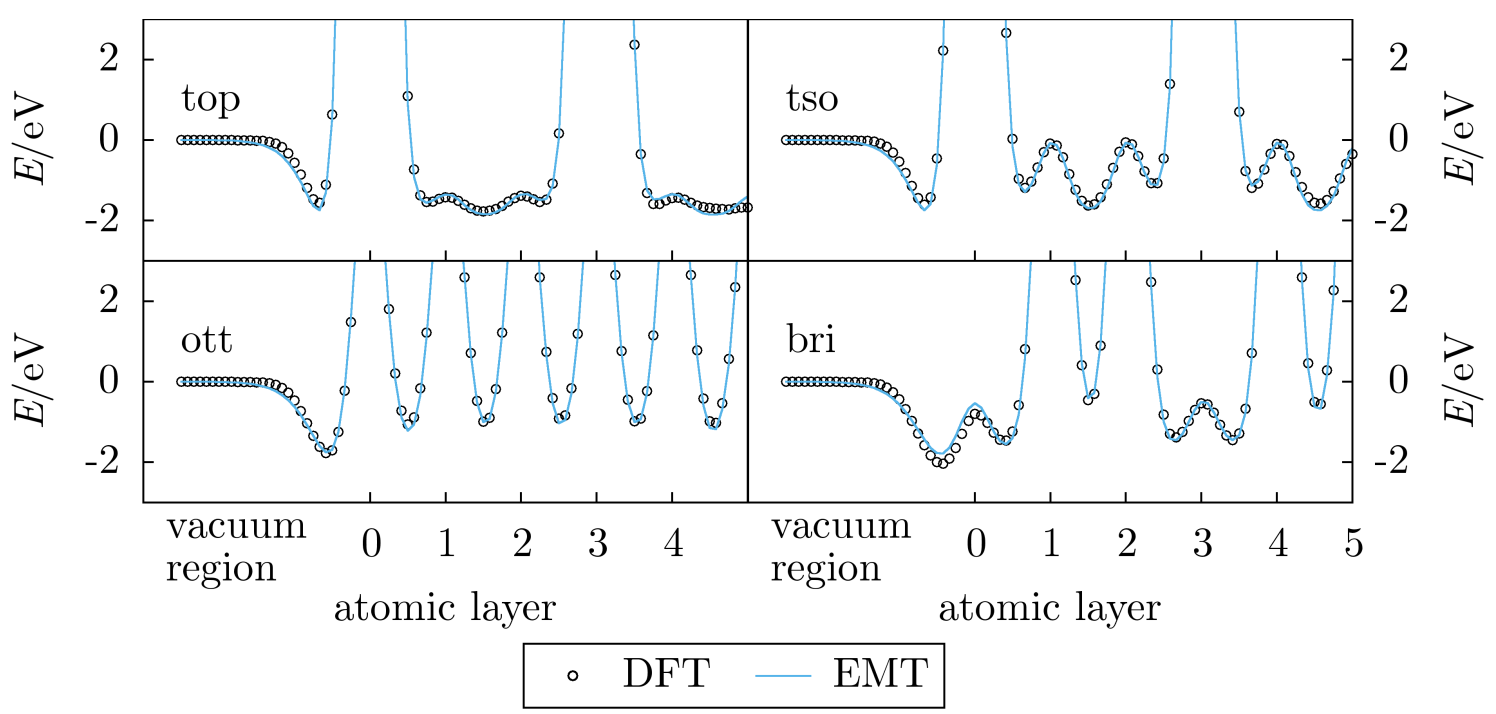

Figure 13: DFT and fitted H-Ag EMT potential energy curves of four high symmetry sites. Black circles show $a b$ initio reference energies and blue curves indicate energies of the parametrized EMT potential.

subsurface minima. Nevertheless, Fig. 13 displays PECs which are in good agreement with the reference data.

The EMT PECs of an $\mathrm{H}$ atom on a $\mathrm{Pd}(111)$ surface is shown in Fig. 14. At first sight, it looks very similar to Fig. 13, but there are subtle differences. The most important difference is that for $\mathrm{Pd}$ the adsorption well is about $1 \mathrm{eV}$ deeper. Even in most subsurface regions, the interaction energy is negative which immediately explains the great interest in palladium for use in hydrogen storage applications. Besides that, the bridge site is again not modeled as well as the other three. One slight drawback of EMT which is visible in both Figs. 13 and 14 is the slope of the PECs towards the chemisorption well above the surface. DFT predicts a steeper descent than the approximations and in consequence the mathematical formulations in EMT cannot reproduce. In any case, it can be expected that the somewhat shallower slope together with the onset of the attractive interaction at too large a distance from the surface is not a problem in the MD calculations. The metal atoms are not influenced because of their large mass and the $\mathrm{H}$ atom energy at the bottom of the chemisorption well is independent of the slope.

In comparison to all other system, $\mathrm{H}$ on $\mathrm{Pt}(111)$ is the most difficult to describe with EMT. The usual PECs are shown in Fig. 15. Most strikingly, the chemisorption well cannot be reproduced by the PES at any of the high symmetry sites. Although Pt has a more attractive well than $\mathrm{Pd}$, one would expect that EMT is capable of reproducing it. After all, the difference in adsorption well depths between Ag and 


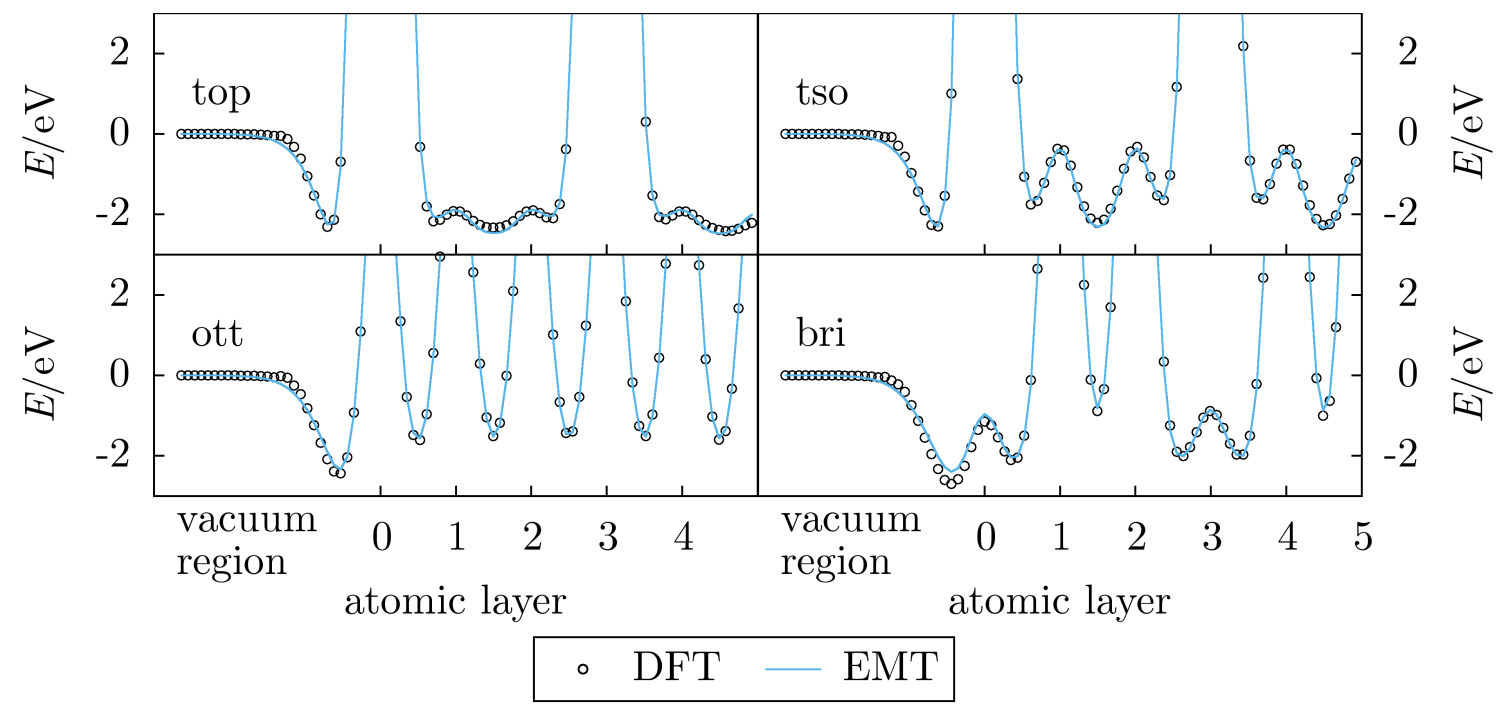

Figure 14: DFT and fitted H-Pd EMT potential energy curves of four high symmetry sites. Black circles show ab initio reference energies and blue curves indicate energies of the parametrized EMT potential.

$\mathrm{Pd}$ is $751 \mathrm{meV}$ at the top-site, while there is only a $340 \mathrm{meV}$ difference between $\mathrm{Pd}$ and $\mathrm{Pt}$. From this point of view there is no rationale why an $\mathrm{H}$ atom can be accurately modeled above a $\mathrm{Ag}$ and $\mathrm{Pd}$ surface, but not above a $\mathrm{Pt}$ surface. The other noteworthy feature is in the Pt subsurface region of the top- and tso-sites. EMT generates a too corrugated PEC at the former and a slightly too corrugated $\mathrm{PEC}$ at the latter high symmetry site. The minimum between the first and second subsurface layer in the top-site panel is the octahedral site (oct). The importance of the oct-site will be commented on in section 4.1.3.

The behavior of this fit is not an exception. All of the best $\mathrm{H}$ on Pt PESs that the GA produced had these two problematic properties. EMT is simply not flexible enough to model a deep adsorption well on the one hand and a repulsive subsurface on the other hand. In consequence, the least squares-based optimization algorithm of course tries to split any deviation evenly between both. $\mathrm{H}$ on $\mathrm{Ag}$ and $\mathrm{Pd}$ could be accurately modeled because the chemisorption well is about as deep as the minima below the surface at the two said surface sites. But it is an entirely different situation at a Pt surface.

Fit to AIMD Trajectories Since all points of the DFT energy grid are used for training the EMT potential, Figs. 13-15 do not contain any information about the predictive capabilities of the respective potentials. About a dozen AIMD trajectories per metal surface were calculated, and for each fit one of them was chosen for 


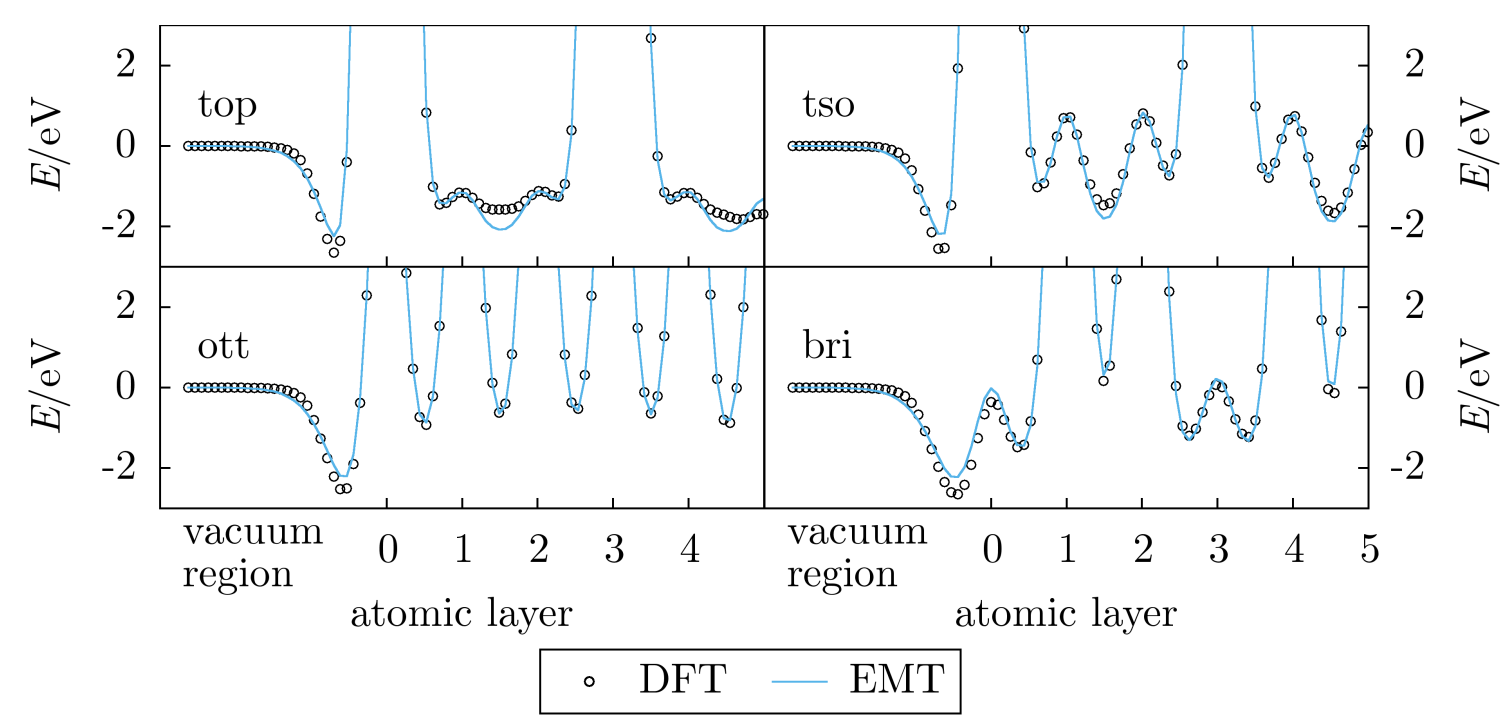

Figure 15: DFT and fitted H-Pt EMT potential energy curves of four high symmetry sites. Black circles show $a b$ initio reference energies and blue curves indicate energies of the parametrized EMT potential.

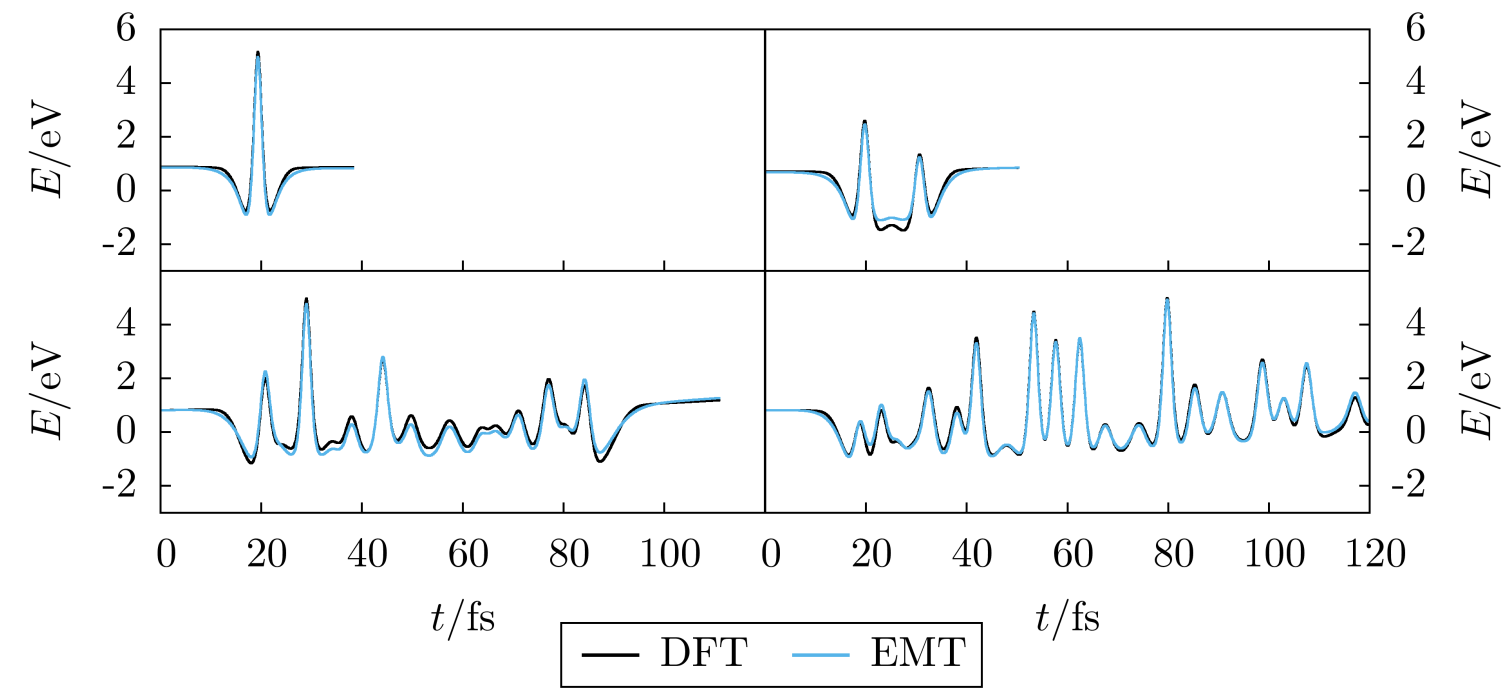

Figure 16: Black lines show the potential energy of the $\mathrm{H}-\mathrm{Ag}(111)$ system during four different AIMD trajectories and scattering events. The blue curves depict energies of the same configurations calculated with the optimized EMT potential.

training. The rest was used as validation data. The RMSE to the validation data is of course more meaningful because it shows how the potential performs when dealing with novel geometries. It is impossible to systematically sample such a high dimensional system. Hence, the AIMD trajectories serve as a (Boltzmann-weighted) indicator of robustness and transferability of the trained EMT potential.

Figs. 16, 17 and 18 show the performance of the optimized H-Ag, H-Pd and H-Pt potentials, respectively, when predicting potential energies from AIMD trajectories. 


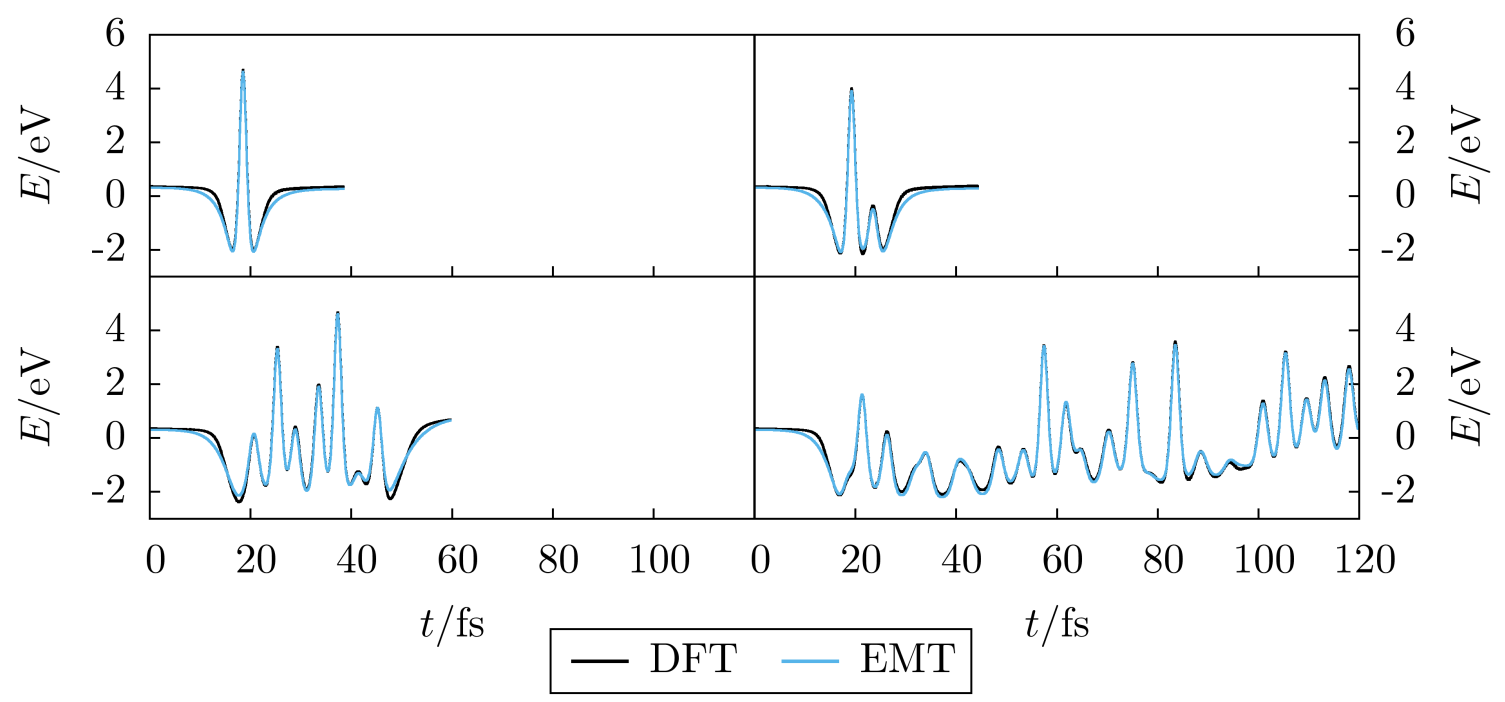

Figure 17: Black lines show the potential energy of the H-Pd(111) system during four different AIMD trajectories and scattering events. The blue curves depict energies of the same configurations calculated with the optimized EMT potential

Each panel shows one trajectory in which the projectile stays subsurface for the entire duration as well as one single-, one double- and one multi-bounce event. One can see the same trends that have already been visible in the DFT grids. Entering the adsorption well, the attractive interaction starts at a slightly too large distance. Apart from that, the $\mathrm{H}-\mathrm{Ag}$ and $\mathrm{H}-\mathrm{Pd} \mathrm{PESs}$ produce accurate results over a more than $5 \mathrm{eV}$ interaction energy range. Especially the high energy peaks in various trajectories are excellently reproduced. The $\mathrm{H}$ on $\mathrm{Pt} \mathrm{PES}$, however, shows again some issues. Minima in the energy are often not captured correctly by the trained potential. However, the general curve shapes match and although the H-Pt PES has the largest RMSE of all trained potentials, the error is still comparable to the systematic DFT error [160].

\subsubsection{Discussion}

From the comparison of the fitted curves to the DFT grid and AIMD trajectories, one can get a crude estimate about the performance of the fits. Tab. 5 now quantifies this deviation from the reference data and adds information about elastic moduli and disintegration temperatures. The shear elastic constant $C_{44}$ can be readily calculated from the fitted EMT parameters via Eq. 2.21. Except for $\mathrm{Al}$ and Rh, all EMT $C_{44}$ values are close to their respective literature value across the board. The penalty function in the GA works well to steer the development over many generations into a direction of maximum fitness. This of course includes the reproduction of supplied 


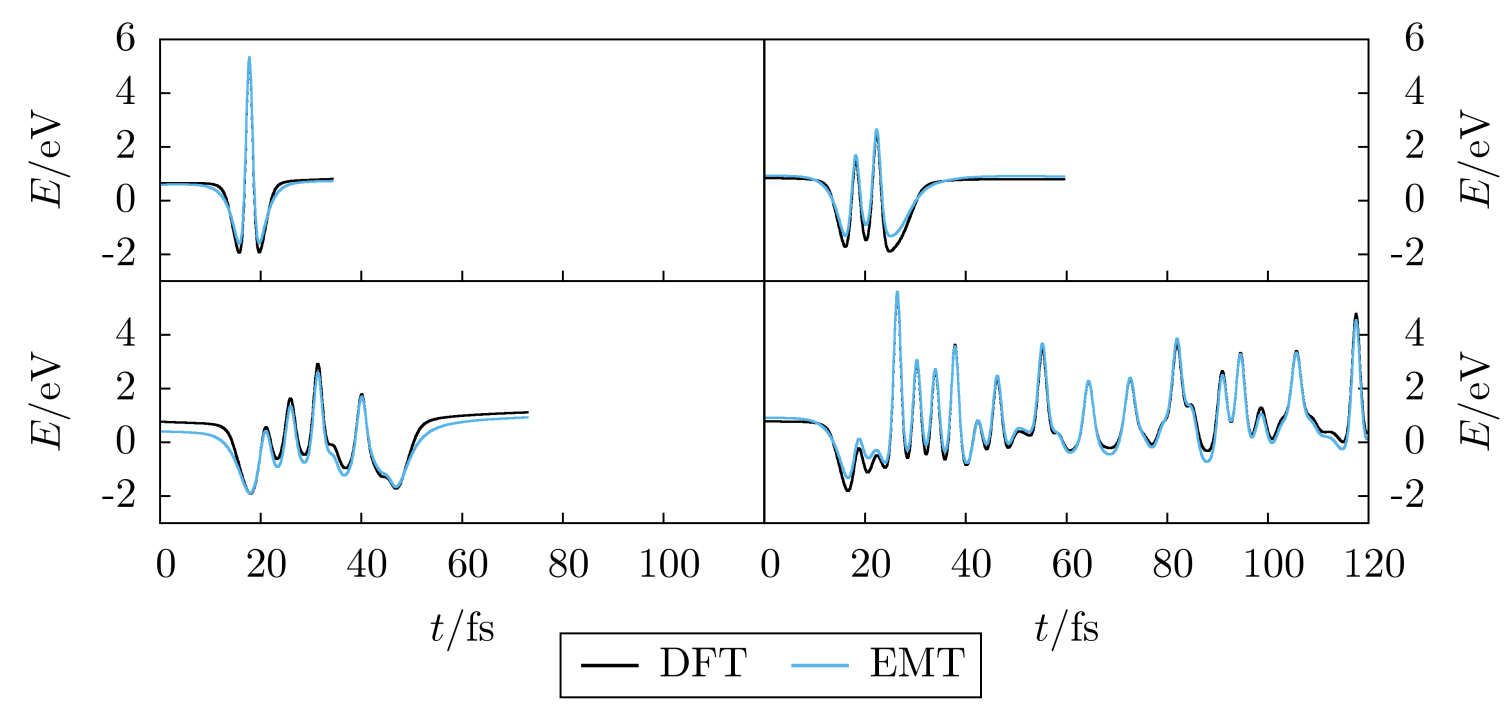

Figure 18: Black lines show the potential energy of the H-Pt(111) system during four different AIMD trajectories and scattering events. The blue curves depict energies of the same configurations calculated with the optimized EMT potential

values from the literature. One real advantage of the GA approach becomes visible in a direct comparison to the previous version of the H-Au PES. S. M. Janke manually set the initial parameters that were fed into the optimization subroutine and arrived at a fit with a disintegration temperature of $1000 \mathrm{~K}$ and a comparable RMSE of $160 \mathrm{meV}$ [80]. The biggest difference is that her $C_{44}$ only amounts to $35.9 \cdot 10^{10} \frac{\mathrm{N}}{\mathrm{m}^{2}}$. Though $C_{44}$ does not directly influence any scattering dynamics of light atoms, it is interesting to note from a methodological point of view that an increase in the shear elastic constant also translates to a higher disintegration temperature.

$\mathrm{H}$ on rhodium is fairly hard to fit for the same reason as $\mathrm{H}$ on platinum. The latter has an adsorption energy difference between the top-site and the subsurface octahedral site (oct) of $1.08 \mathrm{eV}$ while this difference is $0.64 \mathrm{eV}$ in the case of $\mathrm{Rh}$. Thus, the two systems with the highest top-to-oct energy difference also show the largest RMSEs. The coefficient of determination between these two quantities is $R^{2}=0.65$ across all systems and though correlation does not imply causation, it is clearly visible from the PEC at the top-site of Fig. 15 that these are the two most problematic points.

To obtain the disintegration temperature, a surface slab is simulated in the NVTensemble at different temperatures for several tens of picoseconds. The lowest temperature at which the ordered (111) surface structure dissolves is used as $T_{\mathrm{m}}$. This observation proceeds by adatom and vacancy formation and sometimes coincides with the release of one or more atoms into the vacuum if the simulation time is 
Table 5: Summary of properties collected from all parametrized PESs. $T_{\mathrm{m}}$ is the disintegration temperature and $C_{44}$ describes the shear elastic constant. The results are sorted by RMSE in ascending order. Literature values of melting points and all values for $C_{44}$ were found in Ref. [90] except for Rh which was taken from Ref. [161].

\begin{tabular}{rccccc}
\hline metal & $\mathrm{RMSE} / \mathrm{meV}$ & $T_{\mathrm{m}}^{\mathrm{EMT}} / \mathrm{K}$ & $T_{\mathrm{m}}^{\mathrm{lit}} / \mathrm{K}$ & $C_{44}^{\mathrm{EMT}} /\left(10^{10} \frac{\mathrm{N}}{\mathrm{m}^{2}}\right)$ & $C_{44}^{\mathrm{lit}} /\left(10^{10} \frac{\mathrm{N}}{\mathrm{m}^{2}}\right)$ \\
\hline $\mathrm{Al}$ & 134 & 1000 & 933 & 49.2 & 28.3 \\
$\mathrm{Cu}$ & 142 & 1200 & 1358 & 71.1 & 75.7 \\
$\mathrm{Pd}$ & 142 & 1400 & 1828 & 76.7 & 71.7 \\
$\mathrm{Ag}$ & 147 & 900 & 1235 & 44.6 & 46.1 \\
$\mathrm{Au}$ & 156 & 1200 & 1337 & 43.2 & 42.0 \\
$\mathrm{Ni}$ & 179 & 1600 & 1728 & 129 & 124 \\
$\mathrm{Rh}$ & 192 & 2200 & 2237 & 134 & 184 \\
$\mathrm{Pt}$ & 259 & 1600 & 2041 & 79.2 & 76.5 \\
\hline
\end{tabular}

long enough. In my fits, $T_{\mathrm{m}}$ is always below the literature value except for $\mathrm{H}$ on Al. It is surprising at first sight because the shear elastic constants deviate in both directions from literature values and the bulk modulus, which includes the cohesive energy, is fixed to the literature value in all cases. This process called premelting is well documented in the literature. Examples can be found for $\mathrm{Au}[162,163], \mathrm{Al}$ [164], Cu [165], Ag [166], Pt [167] among many more. Premelting has been observed both experimentally and in simulations and is an indication of a physically reasonable potential. Hoss et al. observed the onset of premelting on a $\mathrm{Au}(111)$ surface at $1070 \mathrm{~K}$ which is $80 \%$ of the melting point. Though EMT is a fairly simplistic model, together with the optimized parameters it captures the fact that a solid melts from the outside inwards. The EMT parameter sets for all systems can be found in 6 .

\subsection{Scattering Results}

With so many analytical, full-dimensional PESs at hand, their applicability shall be tested in surface scattering simulations. These types of simulations are valuable because they teach us a lot about mechanisms in small scale energy conversion. Simultaneously, there are ultra-high vacuum apparatus available in this group which can conduct light atom scattering experiments. This is of course a great test for theory and both sides can profit off of it in the end. Topics of research in this section include angular and energy loss distributions, the effect of metal electronic states 
Table 6: Optimized EMT parameters for describing $\mathrm{H}$ on all the discussed (post-) transition metals.

\begin{tabular}{rrrrrrrr}
\hline & $\eta_{2} / \AA^{-1}$ & $n_{0} / \AA^{-3}$ & $E_{0} / \mathrm{eV}$ & $\lambda / \AA^{-1}$ & $V_{0} / \mathrm{eV}$ & $\kappa / \AA^{-1}$ & $s_{0} / \AA^{-1}$ \\
\hline $\mathrm{H}$ & 4.87160 & 0.18102 & -1.97751 & 6.91077 & 0.21350 & 7.50174 & 0.77410 \\
$\mathrm{Al}$ & 3.14835 & 0.06296 & -3.39000 & 2.88874 & 0.56878 & 3.82444 & 1.58273 \\
$\mathrm{H}$ & 5.20958 & 0.11432 & -2.31061 & 7.20569 & 0.33739 & 8.49551 & 0.57941 \\
$\mathrm{Ni}$ & 3.40540 & 0.08876 & -4.44000 & 3.60955 & 5.96071 & 5.90006 & 1.36583 \\
$\mathrm{H}$ & 5.23519 & 0.11321 & -2.12698 & 7.02892 & 0.42658 & 8.80060 & 0.59823 \\
$\mathrm{Cu}$ & 3.30489 & 0.08597 & -3.51000 & 3.65552 & 6.58745 & 5.84225 & 1.42367 \\
$\mathrm{H}$ & 5.11207 & 0.12765 & -2.45383 & 6.83009 & 0.21350 & 9.12625 & 0.75318 \\
$\mathrm{Rh}$ & 3.44968 & 0.05584 & -5.75000 & 4.80446 & 2.71844 & 5.55589 & 1.48424 \\
$\mathrm{H}$ & 4.79284 & 0.11656 & -2.43336 & 6.52787 & 0.41804 & 7.84875 & 0.61153 \\
$\mathrm{Pd}$ & 3.29258 & 0.05533 & -3.90000 & 4.08942 & 3.96130 & 5.67148 & 1.53974 \\
$\mathrm{H}$ & 5.17053 & 0.11365 & -1.99701 & 7.11981 & 0.42048 & 8.16845 & 0.58518 \\
$\mathrm{Ag}$ & 3.29687 & 0.05115 & -2.96000 & 3.59423 & 2.76454 & 5.72616 & 1.62493 \\
$\mathrm{H}$ & 4.92557 & 0.12090 & -2.11450 & 7.01217 & 0.21350 & 8.85208 & 0.64979 \\
$\mathrm{Pt}$ & 3.37380 & 0.06062 & -5.85000 & 4.19626 & 21.6778 & 6.05575 & 1.54677 \\
$\mathrm{H}$ & 5.06612 & 0.13115 & -1.76738 & 8.00198 & 0.21350 & 8.97429 & 0.71031 \\
$\mathrm{Au}$ & 3.16340 & 0.04858 & -3.80000 & 4.12338 & 2.32100 & 5.42918 & 1.64174 \\
\hline & & & & & & &
\end{tabular}

and isotope effects among others.

\subsubsection{Adiabatic Simulation}

Although it is known from the literature [3, 168] that $\mathrm{H}$ atom scattering from clean metal surfaces proceeds anything but electronically adiabatic, it is interesting to see how the interaction would look like if electron-hole pair (ehp) excitations were turned off. Comparisons can then be made to the electronically nonadiabatic treatment in terms of energy loss, sticking probability and scattering angles. Without ehps the expected energy loss of the projectile in one collision is determined by the laws of 


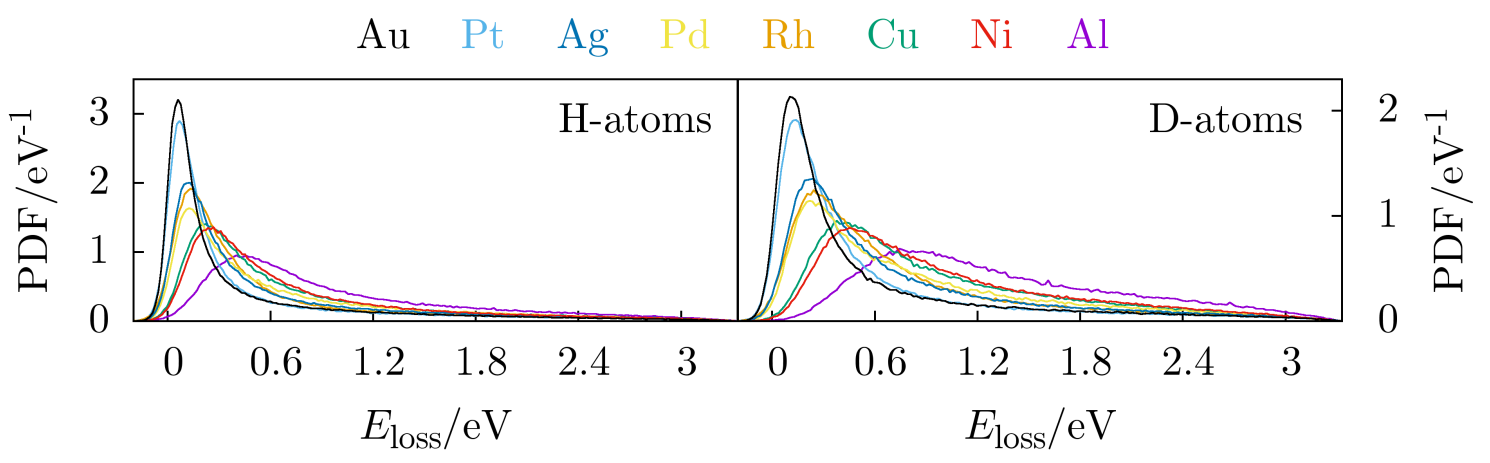

Figure 19: Total energy loss spectra of $\mathrm{H}$ and $\mathrm{D}$ atoms scattering from all parametrized metal surfaces equilibrated to $300 \mathrm{~K}$. The calculations are based purely on Newtonian dynamics and the chemical symbols are sorted by atomic weight in descending order. Incidence conditions are $E_{\mathrm{i}}=3.33 \mathrm{eV}, \theta_{\mathrm{i}}=45^{\circ}$ and $\varphi_{\mathrm{i}}$ points towards the $[10 \overline{1}]$ direction.

energy and momentum conservation

$$
\Delta E=\frac{4 m M}{(m+M)^{2}}\left(E_{\mathrm{i}} \cos ^{2} \theta_{\mathrm{i}}+D\right)-\frac{m(2 M-m)}{(m+M)^{2}} k_{\mathrm{B}} T_{\mathrm{s}}
$$

where $M$ is the effective mass of the surface, $m$ is the mass of the $\mathrm{H}$ atom, $E_{\mathrm{i}}$ is the incidence energy of the projectile, $\theta_{\mathrm{i}}$ is the polar incidence angle, $D$ is the depth of the adsorption well and $T_{\mathrm{s}}$ is the surface temperature. The first term in Eq. 4.2 without the well depth added is the simplest approximation and is known as the Baule limit [169]. The second term describes the influence of thermal motion on the expected energy loss. But it can in fact be neglected in this case because its highest value is assumed in D atom scattering from aluminum and only amounts to $6.4 \mathrm{meV}$ with $T_{\mathrm{s}}=300 \mathrm{~K}$. Since adiabatic simulations are only abstract representations of real systems because they neglect the main energy dissipation channel, for brevity, this section merely contains summarizing aspects of the performed MD calculations. Comparisons to the nonadiabatic case will be presented in greater detail in section 4.2.2.

The first thing to analyze is the total energy loss spectrum. Total refers to an integration over all polar and azimuthal scattering angles as opposed to in-plane which only measures scattered projectiles having the same initial and final azimuthal angle, and specular where both angles are the same before and after collision with the surface. The total energy loss spectra are shown in Fig. 19 for H and D atoms. The color coding indicates various (111) metal surfaces and the ordering of the chemical symbols is based on the atomic weight. As can be expected from the Baule limit, the order of the energy loss peaks reflects nearly perfectly the mass of the 


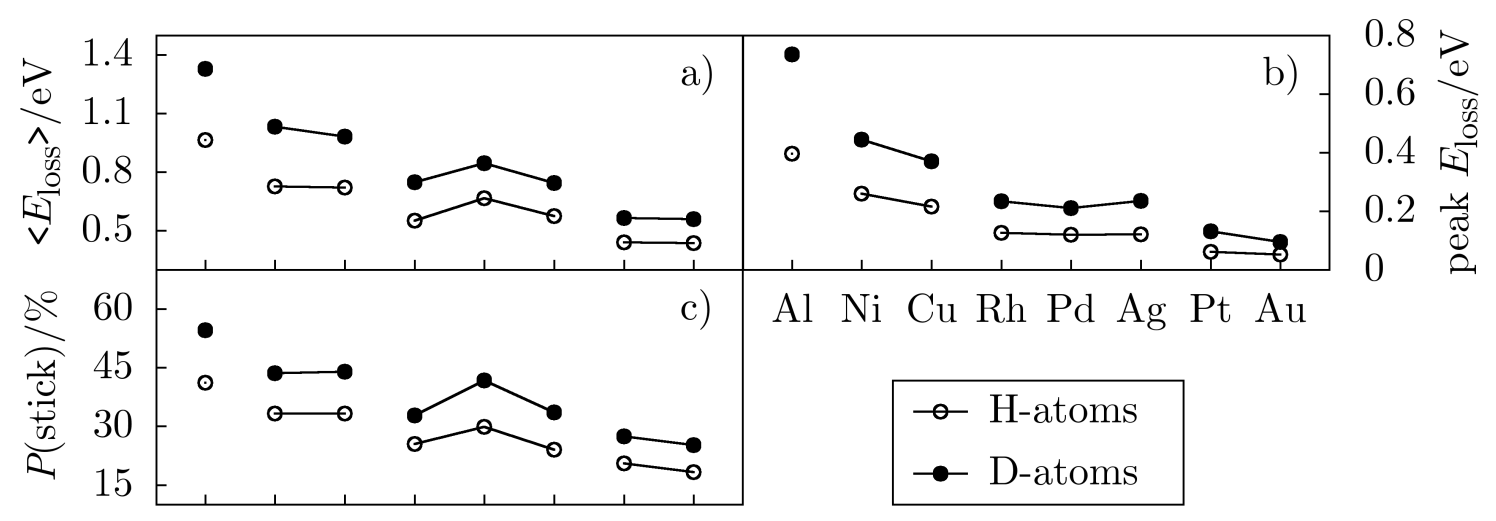

Al Ni Cu Rh Pd Ag Pt $\mathrm{Au}$

Figure 20: Summary of results from electronically adiabatic simulations shown in Fig. 19. Panel a) shows the average and panel b) the most probable energy loss of $\mathrm{H}$ and $\mathrm{D}$ scattering from a variety of metals at $300 \mathrm{~K}$. Panel c) displays the sticking probability. Metals in the same period of the element table are connected by lines.

surface atoms. One can clearly distinguish between aluminum and the $3 d-, 4 d-$ and $5 d$-metals since the peaks are grouped by periods in the table of elements. The only exception to the otherwise ideal arrangement of peak heights is that $\mathrm{Rh}$ and $\mathrm{Pd}$ switched places. But this can be explained by the exceptional absorptive capacity of $\mathrm{Pd}$ for $\mathrm{H}$ atoms. The high energy loss tail of $\mathrm{H}$ on Pd spectra is more pronounced in comparison with its neighbors $\mathrm{Rh}$ and $\mathrm{Ag}$ which leads to a reduced peak height since the area under all curves is normalized to unity. Comparing $\mathrm{H}$ to $\mathrm{D}$ atom scattering, the energy loss spectra are broader in case of $\mathrm{D}$ which can already be seen from the smaller $y$-axis range. They are also shifted to larger energy losses which is immediately comprehensible from the binary collision model.

The energy loss spectra from Fig. 19 are summarized in panels a) and b) of Fig. 20. The average and peak energy loss of $\mathrm{H}$ and $\mathrm{D}$ become smaller the larger the mass of the collision partner. D always loses more energy than $\mathrm{H}$ because of the higher mass ratio between the projectile and the surface. Panel c) shows the sticking probability which strikingly resembles the average energy loss. The results are again neatly ordered by mass with palladium being the only exception due to its pronounced absorptive capacity.

\subsubsection{Nonadiabatic Simulation}

The electronically adiabatic calculations have shown that the parametrized PESs give reasonable results for pure Newtonian dynamics. The propagation method is useful from a theoretical point of view when asking what the energy loss would look 


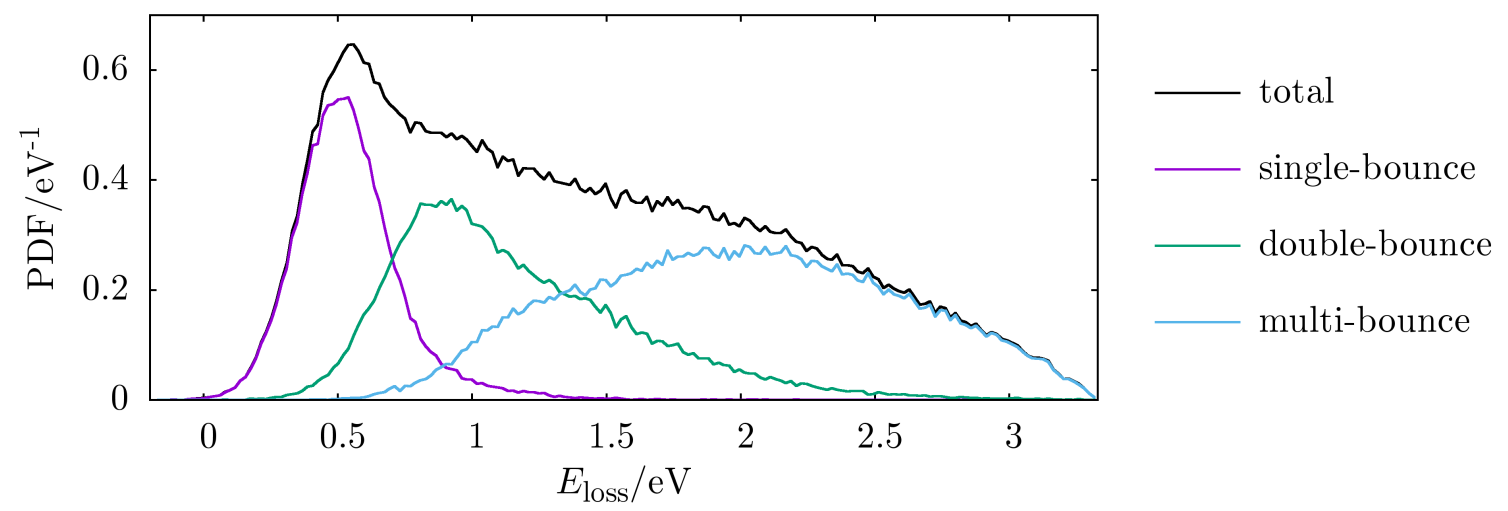

Figure 21: Total energy loss spectrum of $\mathrm{H}$ atoms scattering from a $\mathrm{Ag}(111)$ surface at $300 \mathrm{~K}$ with $E_{\mathrm{i}}=3.33 \mathrm{eV}, \theta_{\mathrm{i}}=45^{\circ}$ and $\varphi_{\mathrm{i}}$ along the $[10 \overline{1}]$ direction. Electron-hole pair excitation is taken into account via the LDFA. The total signal is decomposed into contributions from different bounce events.

like if electron-hole pair excitation did not exist in the interaction between an $\mathrm{H}$ atom and a metal surface. We know from numerous experimental $[2,4,170]$ and theoretical [171-175] sources though that ehps account for the majority of projectile kinetic energy loss. One approach which has successfully been applied by several groups is to replace Newtonian dynamics by Langevin dynamics and to couple the friction coefficient $\eta$ to the electron density (cf. Eq. 2.42). The mapping from the latter to the former is done by a spline function given by Eq. 2.45 and the coefficients can be found in Tab. 1. Each parameter set of an atomic species in EMT contains an $n_{0}$-value which describes the electron density at the center of each nucleus. From the projectile's point of view, the sum of electron density tails of surrounding metal atoms plus its own contribution make up the embedding density that causes the friction in the Langevin equation in the end.

Total energy loss Fig. 21 shows a typical total energy loss spectrum where nonadiabaticity is taken into account via the LDFA. In comparison with the adiabatic energy loss spectra in Fig. 19, there is much more probability density at the high energy loss side. Additionally, different bounce events can be resolved which constitute the total spectrum. Here, single-bounces only account for $23 \%$ of the scattered atoms while double- and multi-bounces make up $32 \%$ and $45 \%$, respectively.

In the style of Fig. 19, Fig. 22 shows the energy loss spectra of $\mathrm{H}$ and $\mathrm{D}$ atoms scattering from a variety of metal (111) surfaces with application of the LDFA. Here, it is more difficult to discern one curve from another. Especially in the high energy loss regime of the left panel all curves nearly overlap. In the rising part of the curves, one can still distinguish the different periods of the element table. The separation 


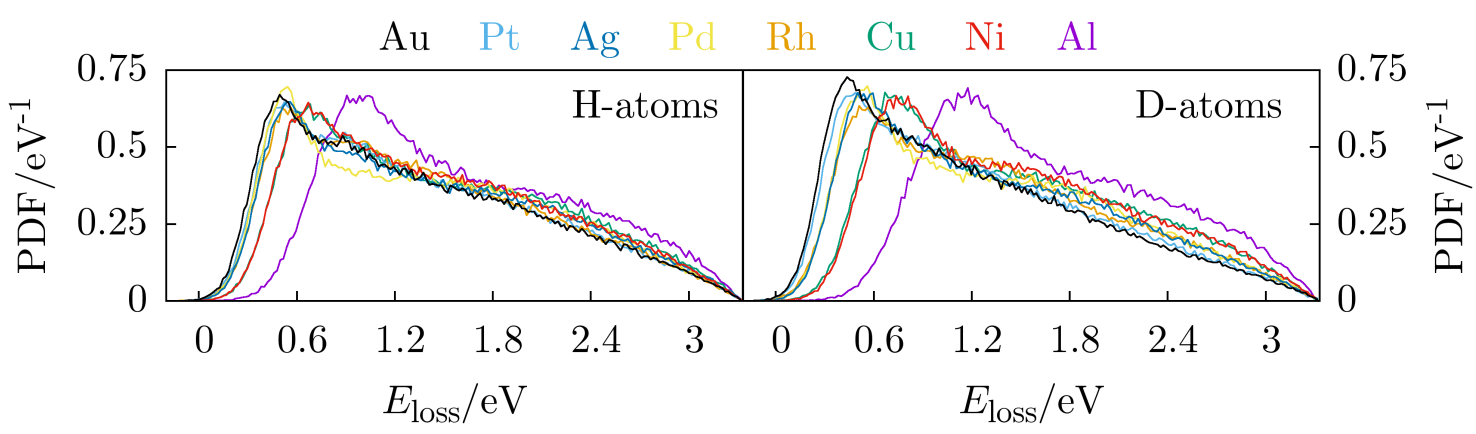

Figure 22: Total energy loss spectra of $\mathrm{H}$ and D atoms scattering from all parametrized metal surfaces thermalized to $300 \mathrm{~K}$. Incidence conditions are $E_{\mathrm{i}}=3.33 \mathrm{eV}, \theta_{\mathrm{i}}=45^{\circ}$ and $\varphi_{\mathrm{i}}$ along the $[10 \overline{1}]$ direction. Electron-hole pair excitation is taken into account by the LDFA. The chemical symbols are sorted by atomic weight in descending order which still matches the peak ordering from left to right.

is more distinct in the right panel because of the higher mass of $\mathrm{D}$ atoms. Apart from that, the spectra of $\mathrm{H}$ and $\mathrm{D}$ look more similar than in the adiabatic case. This is what I expected to see since this phenomenon has already been described in Ref. [23] and can be explained in the following way: inserting the masses of $\mathrm{H}$ and D into Eq. 4.2 and assuming a large surface atomic weight, one arrives at an energy loss for $\mathrm{D}$ atoms that is twice as high as for $\mathrm{H}$ atoms. This is the limiting case as there are several factors which decrease this ratio. For example, it is still 1.98 with $\mathrm{Au}$ as a collision partner, but only 1.86 is the case of Al. This ratio can be further diminished be multiple bounces which projectiles are likely to undergo in a typical trajectory. Next, at least experimentally, $\mathrm{H}$ and $\mathrm{D}$ atoms do not have the same incidence kinetic energies because they are created from ultraviolet laser photolysis of HI. The HI center of mass motion needs to be preserved after dissociation hence $\mathrm{H}$ atoms gain more kinetic energy than $\mathrm{D}$ atoms. This fact is taken into account in the simulations and further decreases the ratio of D to H energy losses.

The most important effect though, is the frictional force exerted on the incoming particles in the nonadiabatic calculations. It is opposite of the adiabatic isotope effect. Since friction in the LDFA is only dependent on the embedding electron density which is the same for $\mathrm{H}$ and $\mathrm{D}$, it decelerates $\mathrm{H}$ more than $\mathrm{D}$ by a factor of $\sqrt{m_{\mathrm{D}} / m_{\mathrm{H}}} \approx 1.41$. In summary, the energy loss spectra of $\mathrm{H}$ and $\mathrm{D}$ in Fig. 22 look fairly similar because the adiabatic and nonadiabatic isotope effects work in opposite directions and nearly cancel each other. This fact is illustrated in Fig. 23. With the exception of $\mathrm{Al}(111)$, the average and peak energy loss of $\mathrm{H}$ and $\mathrm{D}$ are remarkably similar. In fact, the average energy loss is equal at these incidence conditions for the $3 d$ metals. The lighter metal shows a larger energy loss for D 


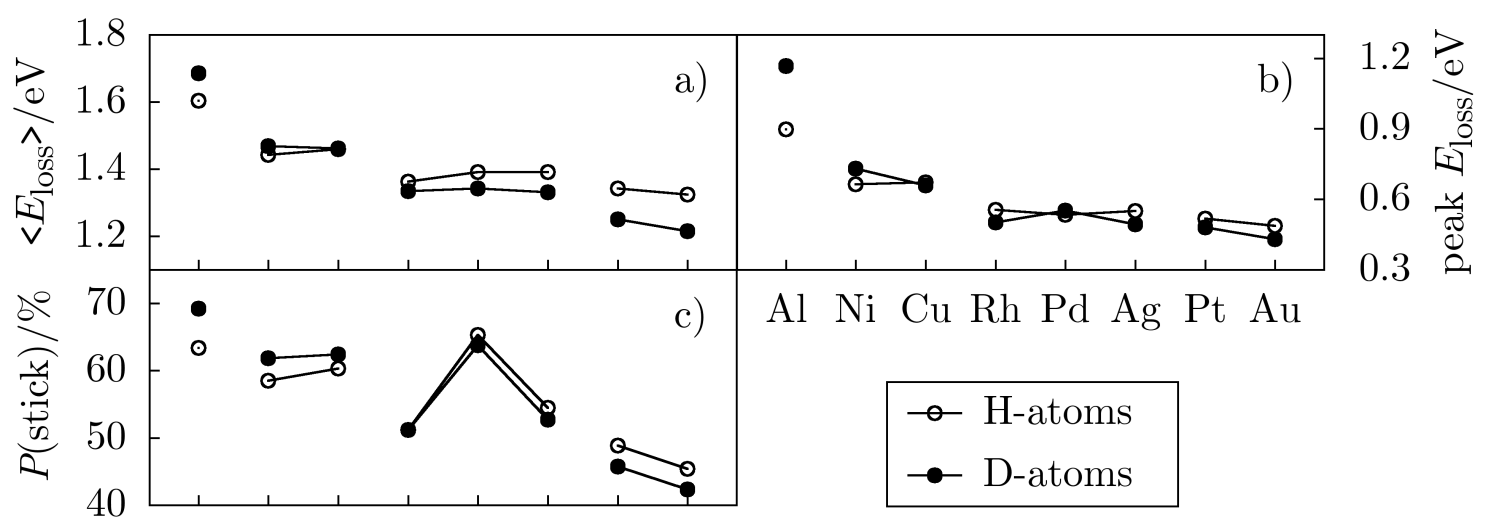

$\mathrm{Al} \mathrm{Ni} \mathrm{Cu} \mathrm{Rh} \mathrm{Pd} \mathrm{Ag} \mathrm{Pt} \mathrm{Au}$

Figure 23: Overview of certain projectile-metal interaction characteristics when ehp excitation is taken into account via the LDFA. Panel a) shows average energy losses of H and D atoms scattering from different (111) metal surfaces at $300 \mathrm{~K}$. Panel b) displays their most probable energy loss. The sticking probability is shown in panel c). Incidence conditions are $E_{\mathrm{i}}=3.33 \mathrm{eV}, \theta_{\mathrm{i}}=45^{\circ}$ and $\varphi_{\mathrm{i}}$ along the $[10 \overline{1}]$ direction.

Figure 24: $\mathrm{H}$ and $\mathrm{D}$ energy loss to phonons and electronhole pairs when scattering off of a variety of metal (111) surfaces at $300 \mathrm{~K}$. Nonadiabatic energy loss calculated by means of the LDFA. Incidence conditions are $E_{\mathrm{i}}=3.33 \mathrm{eV}, \theta_{\mathrm{i}}=$ $45^{\circ}$ and $\varphi_{\mathrm{i}}$ along the $[10 \overline{1}] \mathrm{di}-$ rection. Empty and filled circles describe $\mathrm{H}$ and $\mathrm{D}$ atoms, respectively. Phononic energy loss is indicated in purple and ehps are marked in green.

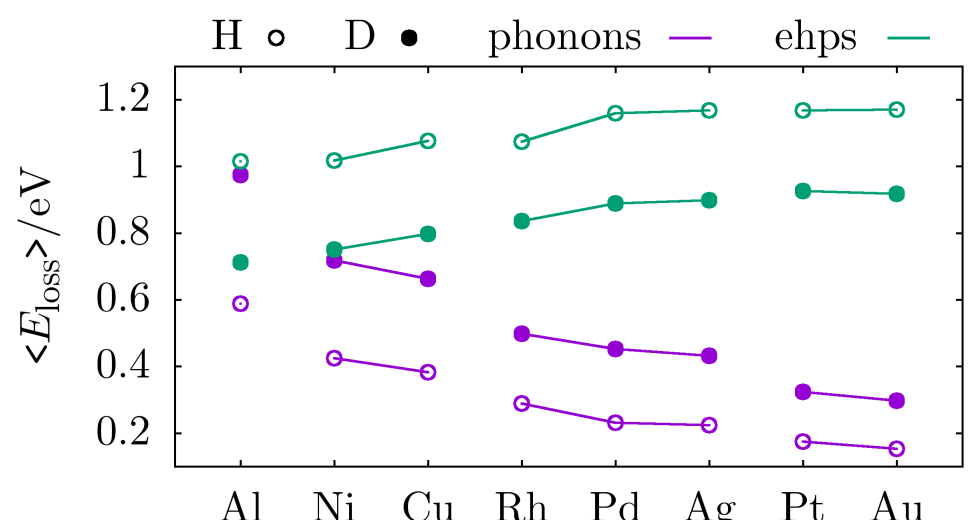

Al $\mathrm{Ni} \quad \mathrm{Cu}$ Rh $\mathrm{Pd}$ Ag $\mathrm{Pt} \quad \mathrm{Au}$

whereas the simulations predict $\mathrm{H}$ to lose more energy on average when colliding with metals located in the fourth and fifth period of the element table. The sequence in Fig. 23b) behaves similarly with the only difference that the point of equal $\mathrm{H}$ and $\mathrm{D}$ energy loss is located at the $4 d$ metals. A preference for $\mathrm{D}$ in collisions with light metals that at some point switches to a preference for $\mathrm{H}$ when colliding with heavy metals is also observed in the sticking probability. Again, palladium stands out with a large adsorptive capacity for $\mathrm{H}$ and $\mathrm{D}$ which resembles that of aluminum although $\mathrm{Pd}$ is a $4 d$ metal.

Energy dissipation channels The energy loss can be further subdivided to better understand the striking similarity between $\mathrm{H}$ and D. Fig. 24 splits the dissipated 


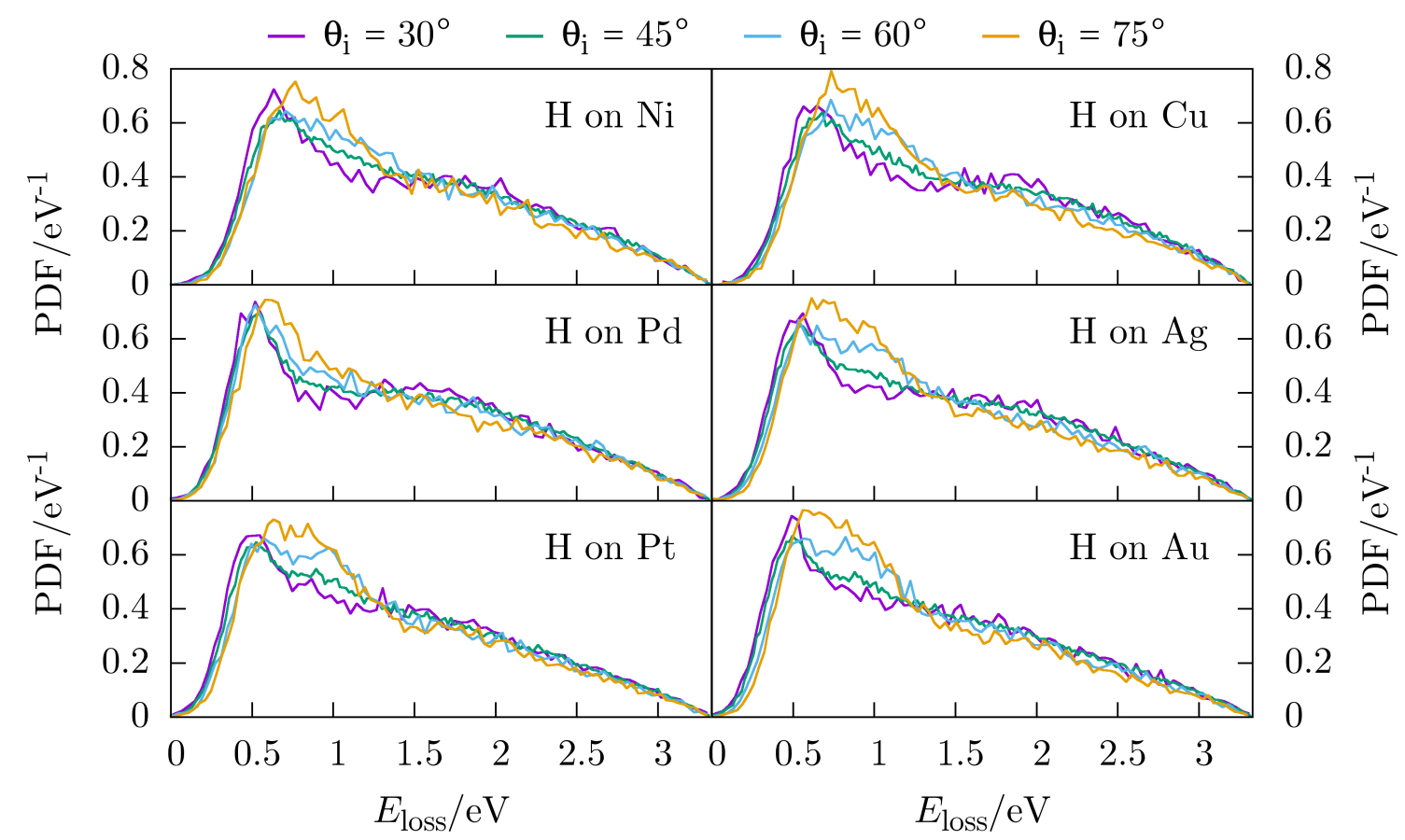

Figure 25: Total energy loss spectra of H scattering off of several (111) surfaces at $300 \mathrm{~K}$. Incidence

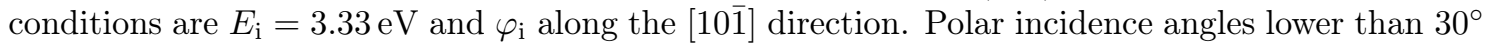
or higher than $75^{\circ}$ do not visibly change the spectrum.

energy into phononic and electronic parts. In all cases, $\mathrm{H}$ atoms lose more energy to ehps than D atoms for reasons explained in the preceding paragraph. Also note that the difference between $\mathrm{H}$ and $\mathrm{D}$ energy losses to ehps stays approximately the same regardless of the collision partner. On the other hand, D atoms always dissipate more energy to phonons. Here, the larger the atomic weight of the metal, the smaller the difference between $\mathrm{H}$ and $\mathrm{D}$ which makes sense with the binary collision model in mind. When taking both of these effects into account, it becomes clear why the two isotopes show a comparable energy loss no matter what metal atom they are colliding with. The different scaling of the effects also explains why metal mass and average energy loss are inversely proportional to one another as illustrated in Fig. 23a).

Incidence angle effect So far, all presented results were obtained at a polar incidence angle of $\theta_{\mathrm{i}}=45^{\circ}$. Fig. 25 shows what happens to the total energy loss spectrum when the incidence energy is fixed at $3.33 \mathrm{eV}$, but the incidence angle is varied. All metals show a similar behavior. The displayed change in the structure of the spectrum can be explained in the following way: at $\theta_{\mathrm{i}}=75^{\circ}$, the already closed (111) surface gives the projectile barely any opportunity to go subsurface. There- 


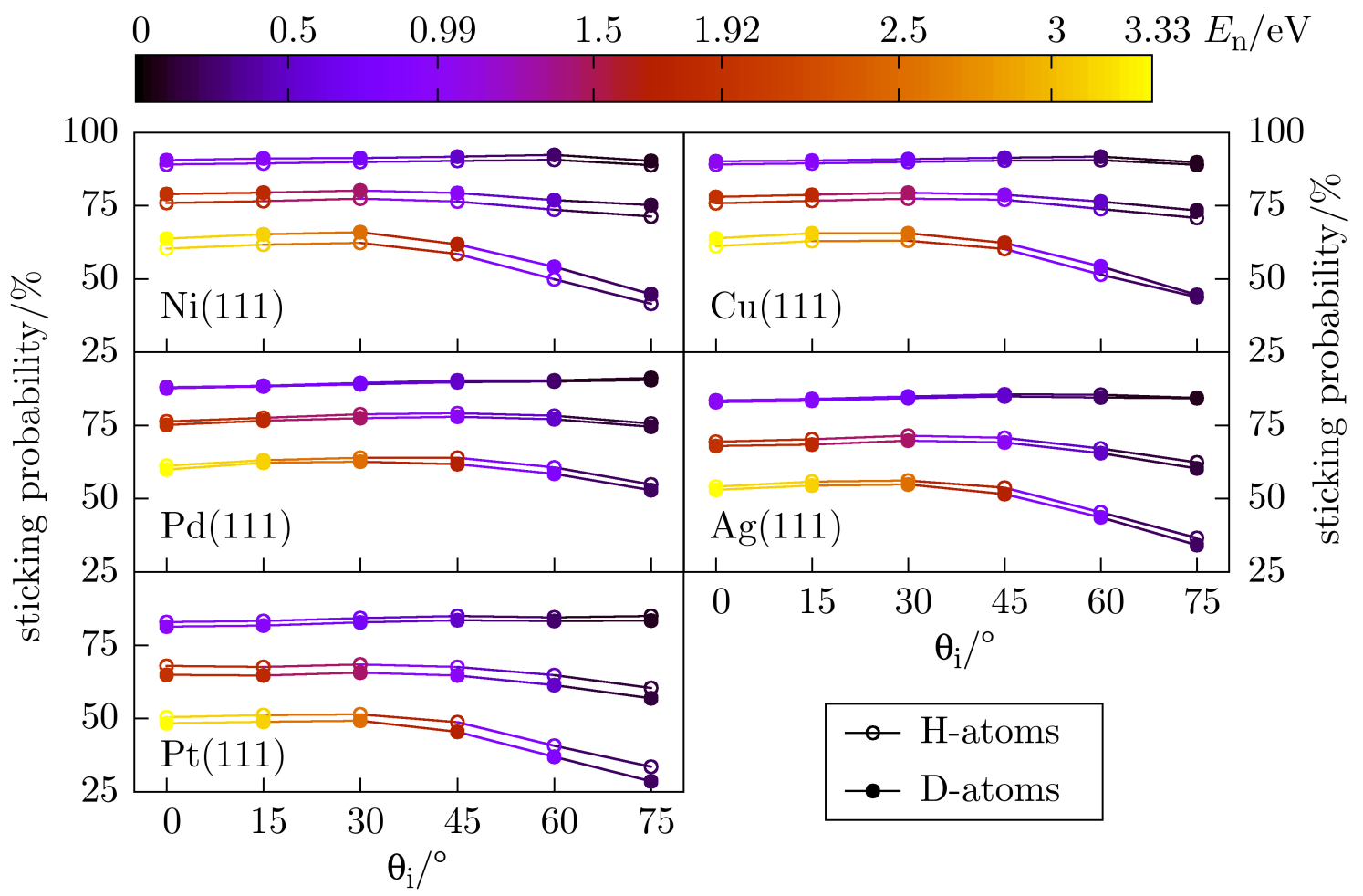

Figure 26: Sticking probabilities of H and D atoms on metal (111) surfaces from different periods of the table of elements in dependence on the polar incidence angle $\theta_{\mathrm{i}}$. The surfaces were thermalized to $300 \mathrm{~K}$. Incidence energies of $E_{\mathrm{i}}=0.99,1.92$ and $3.33 \mathrm{eV}$ were used and the color coding indicates the energy component normal to the surface. Data points obtained with the same $E_{\mathrm{i}}$ are connected by lines.

fore, the projectiles are likely to scatter after one or two bounces. Of all scattered $\mathrm{H}$ atoms, $66 \%$ experienced one or two bounces when colliding with $\mathrm{Cu}(111)$ and $60 \%$ when $\operatorname{Ag}(111)$ was their collision partner. Hence, the probability density function is higher at small energy losses due to a small number of bounces if the angle of incidence is high. At $\theta_{\mathrm{i}}=30^{\circ}$, the surface appears much more open to the impinging particles. In this case, $\mathrm{H}$ atoms scattering from $\mathrm{Cu}$ have a probability of $52 \%$ for single- or double-bounce events and $45 \%$ when colliding with Ag. This leaves more room for multi-bounce trajectories leading to more signal at larger energy losses. These observations are in line with calculations from S. M. Janke of $\mathrm{H}$ on $\mathrm{Au}(111)$ who found a "lowering of the probability to experience multibounce events at high polar angles" [80].

Sticking probability The next property to look at is the sticking probability of $\mathrm{H}$ and D atoms in dependence on polar incidence angle and incidence energy. Fig. 26 provides an overview about absorption into five different metal surfaces. The sticking 


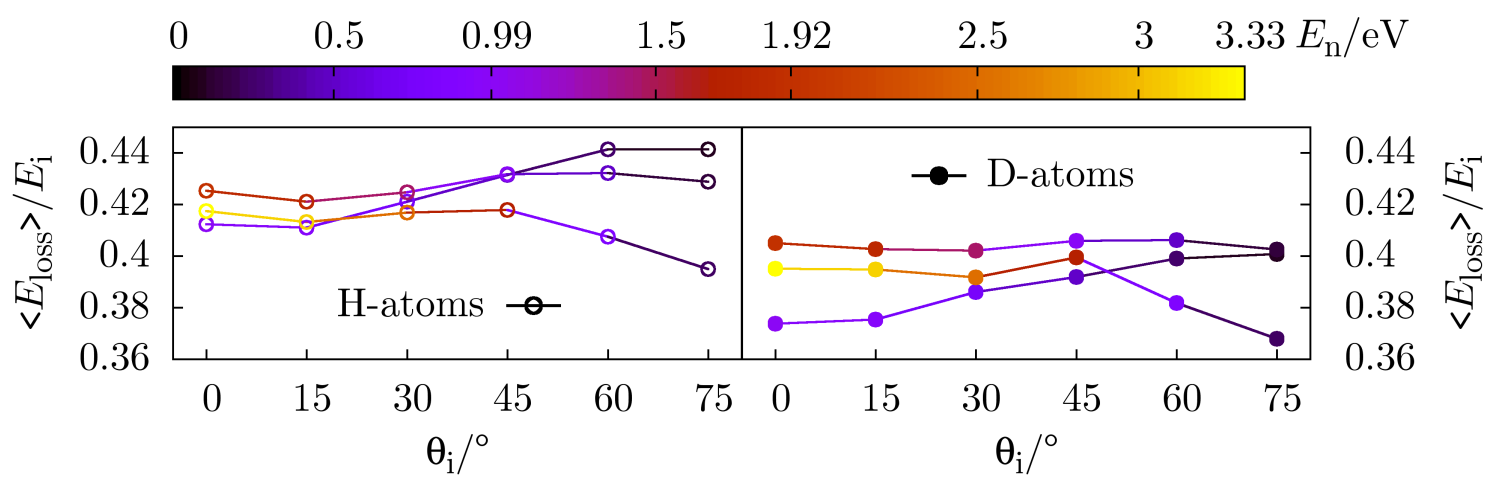

Figure 27: Fractional total energy loss of $\mathrm{H}$ and $\mathrm{D}$ atoms scattering from a silver(111) surface at $300 \mathrm{~K}$. The incidence energies are $E_{\mathrm{i}}=0.99,1.92$ and $3.33 \mathrm{eV}$ and the azimuthal direction is along $[10 \overline{1}]$. The color coding illustrates the incidence energy normal to the surface.

probabilities of both isotopes are again very similar at all angles and energies. They are indistinguishable at $E_{\mathrm{i}}=0.99 \mathrm{eV}$ and only slight differences become apparent at higher incidence energies. Note that on nickel and copper D always has an equal or higher chance than $\mathrm{H}$ of becoming trapped, while it is the other way round on silver and platinum. In any case, since there is no barrier to adsorption on any metal surface (cf. Fig. 9), the lower the incidence energy, the higher the sticking probability. At $E_{\mathrm{i}}=0.99 \mathrm{eV}$, the trapping probability seems to be independent of the incidence angle. If the impinging particle is provided with more energy though, there is a decrease in trapping at high polar angles. The reason is similar to the one causing the shifts in the energy loss spectrum discussed in the previous paragraph. Because there is an attractive force between metal and $\mathrm{H}$ atom at each high symmetry site, the projectile is always pulled towards it. The slower it is, the more its trajectory can be influenced which explains the nearly horizontal line at $E_{\mathrm{i}}=0.99 \mathrm{eV}$. There is even a slight upwards slope in the case of $\mathrm{Pd}(111)$ which is known to act like a sponge for $\mathrm{H}$ atoms. The drop in observed trapping at $E_{\mathrm{i}}=3.33 \mathrm{eV}$ and high polar angles is the result of the particle being least susceptible to course changes and the metal atoms themselves geometrically blocking access to adsorption channels into the bulk.

Fractional energy loss In 2015, Bünermann and coworkers reported that $\mathrm{H}$ atoms scattering from $\mathrm{Au}(111)$ lose the same fraction of their initial translational energy [168]. Whether the projectile is provided with $E_{\mathrm{i}}=0.99$ or $3.33 \mathrm{eV}$, they found that it loses $(33 \pm 1) \%$ on average in case $\theta_{\mathrm{i}}=\theta_{\mathrm{f}}=45^{\circ}$. This result is supported by MD simulations taking ehp excitations into account on the level of the LDFA. Here, I want to analyze how the fractional energy loss depends on isotope substitution, 
Table 7: Average fractional energy losses in percent from $\mathrm{H}$ and $\mathrm{D}$ atom scattering simulations using all parametrized EMT PESs. The numbers are extracted from simulated specular energy loss spectra with $\theta_{\mathrm{i}}=\theta_{\mathrm{f}}=45^{\circ}$ at three incidence energies. The metal slabs are at room temperature and the simulated detector has a radius of $5^{\circ}$ solid angle. The standard error of the estimate is not higher than one percentage point.

\begin{tabular}{ccccccc}
\hline & \multicolumn{7}{c}{ incidence energy and projectile } \\
metal surface & $0.99 \mathrm{eV}$ & $0.94 \mathrm{eV}$ & $1.92 \mathrm{eV}$ & $1.87 \mathrm{eV}$ & $3.33 \mathrm{eV}$ & $3.27 \mathrm{eV}$ \\
& $\mathrm{H}$ & $\mathrm{D}$ & $\mathrm{H}$ & $\mathrm{D}$ & $\mathrm{H}$ & $\mathrm{D}$ \\
\hline $\mathrm{Al}$ & 44.7 & 44.7 & 44.6 & 45.0 & 44.1 & 44.5 \\
$\mathrm{Ni}$ & 40.0 & 40.5 & 41.4 & 40.8 & 40.8 & 40.0 \\
$\mathrm{Cu}$ & 41.5 & 40.9 & 42.0 & 40.0 & 40.9 & 39.4 \\
$\mathrm{Rh}$ & 41.0 & 38.3 & 40.2 & 37.9 & 38.8 & 36.7 \\
$\mathrm{Pd}$ & 39.9 & 35.9 & 38.3 & 35.5 & 38.5 & 36.4 \\
$\mathrm{Ag}$ & 40.1 & 37.8 & 40.9 & 37.6 & 39.1 & 36.5 \\
$\mathrm{Pt}$ & 40.0 & 36.0 & 39.0 & 36.1 & 38.2 & 34.7 \\
$\mathrm{Au}$ & 38.5 & 36.3 & 38.5 & 36.1 & 36.3 & 33.5 \\
\hline
\end{tabular}

metal surface and polar incidence energy. Fig. 27 shows the fractional energy loss of $\mathrm{H}$ and $\mathrm{D}$ scattering from a $\mathrm{Ag}(111)$ surface. Three different incidence energies were used and $\mathrm{H}$ atoms lost $(40 \pm 1) \%$ and $\mathrm{D}$ atoms $(39 \pm 1) \%$ of their initial kinetic energy on average. It is remarkable that this energy loss stays nearly constant over such a wide range of energies and incidence angles. Changing the surface does not change the overall results. The total amount of lost energy increases the lighter the metal as was shown in Fig. 22. But the spread stays nearly the same.

This observation still persists when looking at specular energy loss spectra. Some numbers showing the general behavior are presented in Tab. 7. The numbers in one row are very similar to each other with the largest spread being 2.3 percentage points. This reflects the results of the mean fractional energy loss from all scattered particles shown in Fig. 27 and agrees with the published results of Bünermann et al.. The provided values in Tab. 7 are based on one million trajectories each. Such a large amount of simulations is necessary to reach an accuracy of 0.5 percentage points. Scattering off of $\mathrm{Cu}(111)$ at low incidence energies is the most difficult case. In this system, only $0.08 \%$ of all launched projectiles hit the simulated detector even with a comparatively large radius of $5^{\circ}$ solid angle. 


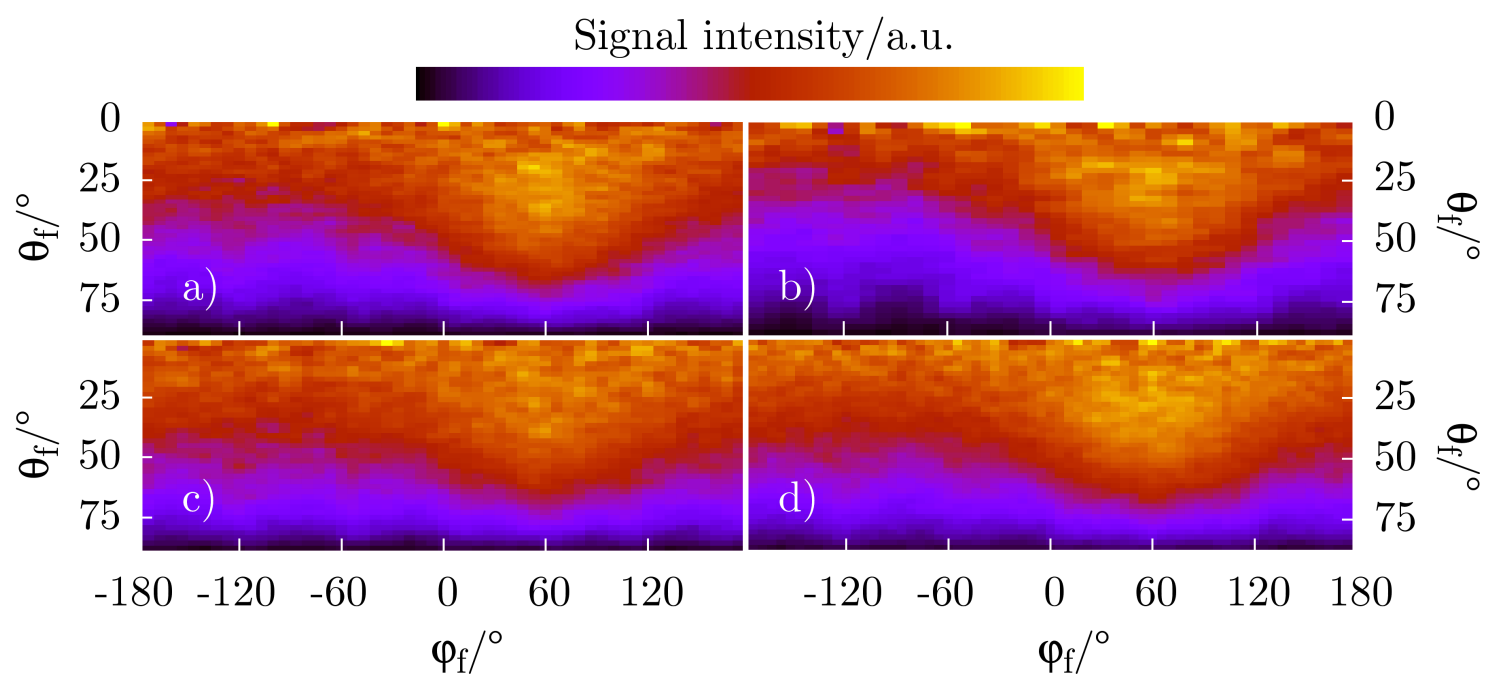

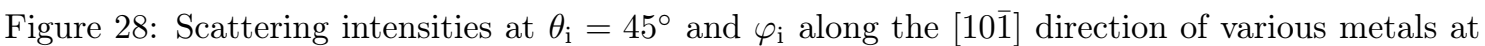
room temperature. Panel a) shows the $\mathrm{H}$ atom scattering signal from $\mathrm{Al}(111)$ at $E_{\mathrm{i}}=1.92 \mathrm{eV}$, b) shows $\mathrm{D}$ atoms scattering from $\mathrm{Cu}(111)$ at $E_{\mathrm{i}}=0.99 \mathrm{eV}, \mathrm{c}$ ) shows $\mathrm{H}$ atoms scattering from $\mathrm{Ag}(111)$ at $E_{\mathrm{i}}=3.33 \mathrm{eV}$ and $\mathrm{d}$ ) shows $\mathrm{D}$ atoms scattering from $\mathrm{Au}(111)$ at $E_{\mathrm{i}}=1.92 \mathrm{eV}$. The signal is scaled to compensate for different sticking probabilities. Metals and incidence conditions were chosen such that they are as diverse as possible.

Angle-resolved Signal Strength This section about simulated, nonadiabatic scattering ends with two interesting observations that I made during the analyses. One question from experimentalists that usually arises at some point during discussions is where to put the detector to observe some interesting behavior of the scattered particles. Since in theory it is straightforward to build a detector with arbitrary resolution covering all scattering polar and azimuthal angles, their question should be easy to answer with a functional full-dimensional PES at hand.

Fig. 28 shows scattering intensities of $\mathrm{H}$ and D from different metal (111) surfaces. The surface atom mass ranges from 27 to $197 \mathrm{u}$ and incidence energies cover a range of over $2.3 \mathrm{eV}$. Scaling the intensities is necessary in this figure to account for different sticking probabilities of the utilized metals at various incidence conditions. The specular direction is $\theta_{\mathrm{f}}=45^{\circ}$ and $\varphi_{\mathrm{f}}=60^{\circ}$ which is the region of highest signal in all systems. Notwithstanding very different incidence conditions and the use of two isotopes, the panels in Fig. 28 look very much alike. Most signal can be found in the forward scattering direction, but quite a number of projectiles also experience backscattering. Continuing the central theme of this section, once more we see that $\mathrm{H}$ and $\mathrm{D}$ atoms on fcc metal (111) surfaces have way more in common than one initially would think. 
Figure 29: Image with subatomic resolution of the $\mathrm{Pt}(111)$ surface at room temperature when $\mathrm{H}$ atom energy loss is mapped onto a color spectrum. The contour dimension shows the fraction of the retained projectile kinetic energy. Bright spots reflecting the hexagonal surface structure indicate on-top hits leading to single bounce events and a consequently small energy loss. Incidence conditions are $E_{\mathrm{i}}=3.33 \mathrm{eV}, \theta_{\mathrm{i}}=45^{\circ}$

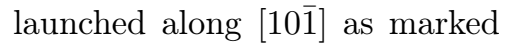
by the white arrow. Other directions are provided for orientation.

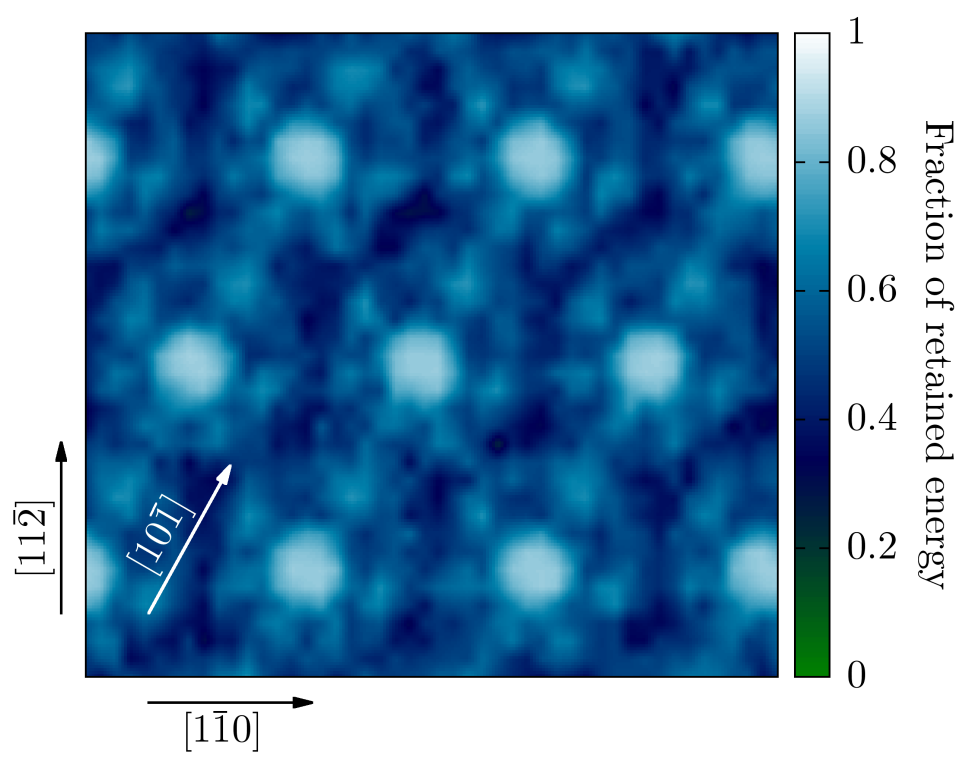

Energy Loss on the sub-atomic Scale Another plot worth mentioning is shown in Fig. 29. In this heat map, the fraction of retained energy of $\mathrm{H}$ atoms scattering from a $\operatorname{Pt}(111)$ surface is shown in dependence on their initial position above the surface. Incidence conditions are provided in the caption. The hexagonal structure of the (111) surface is clearly visible. On-top hits are responsible for the bright spots indicated by a small energy loss. This feature is produced by single bounce events as explained in Fig. 21.

Some atoms hit at the hcp or fcc hollow site. In this case the $\mathrm{H}$ atoms collide with Pt-atoms in the first or second subsurface layer. They will experience multiple bounces and lose a large portion of their initial energy. Some of them will not be seen as signal because they thermalize with the slab before they can escape. Other will flee the bulk and are visible as darker areas in Fig. 29.

The last feature that can be extracted from this plot are the less bright spots of which there are six surrounding the main ones. These smaller spots are not equally distributed around the atom center. That is exactly what one would expect if the particle source were at a polar angle of $45^{\circ}$. This feature arises from double bounce events. These incident particles lose more energy than those hitting directly on top of a Pt-atom, but not as much as the ones going subsurface. Since the H atom incidence energy in this figure is $3.33 \mathrm{eV}$, they are reflected from one surface atom towards its neighbor. Not too much ehp excitation can take place in this short amount of time. Subsequent to the second collision, the projectiles are scattered away from the surface and detected. 


\subsubsection{Comparison with Experiment}

All of the theoretical results presented in the preceding section are puzzle pieces towards understanding the large energy loss of the particles and the role of electronhole pair excitation. Using the LDFA, the results change drastically in comparison with purely classical dynamics. At least for $\mathrm{H}$ on $\mathrm{Au}(111)$, adiabatic simulations fail spectacularly in predicting the energy loss when compared with experimentally obtained data. Furthermore, splitting the dissipated energy into phononic and electronic contributions explains why the scattering results from different metals look so similar. Simultaneously, the nearly non-existing isotope effect can be understood by considering two compensating effects which was known to be valid for $\mathrm{H}$ and $\mathrm{D}$ scattering from $\mathrm{Au}(111)$ prior to my work [80].

Thanks to Bünermann and coworkers, the theoretically predicted energy loss spectra have to a large extent been studied in experiment. This is the acid test for the entire machinery described in this section about scattering from metal surfaces. I started with carefully chosen DFT grid points above and inside the metal slab in combination with basically random non-equilibrium data from AIMD calculations. These datasets were subsequently absorbed by the genetic algorithm trying to breed the fittest PES, whose gradients superimposed by, yet another, parametrized friction force should finally be able to reproduce experimental findings.

The direct comparison of specular energy loss spectra of both hydrogen isotopes scattering from six different fcc metals is shown in Figs. 30 and 31. Three different incidence energies are displayed side by side while the various metal surfaces are stacked on top of each other. Experimentally obtained data points are marked by black dots and the red line denotes the calculated energy loss. In experiment, the detector has a diameter of $3.18^{\circ}$ solid angle whereas theory uses $10^{\circ}$. The advantage is simply the nearly 10 times larger detector area in the simulations which is necessary to achieve a reasonable signal-to-noise ratio without making the binning interval ridiculously large. At the same time, it still only collects signal from forward scattered atoms located in the high intensity region as shown in Fig. 28.

Increasing the detector size in comparison to experiment is also necessary to keep the computational demands in realistic dimensions. Each panel of Figs. 30 and 31 is the result of one million simulated trajectories. In the worst case which is D atoms scattering from $\mathrm{Cu}(111)$ at $E_{\mathrm{i}}=0.94 \mathrm{eV}$, only 773 particles of them actually reach the simulated detector. This is because only $8.66 \%$ of the incoming particles are reflected of which $0.893 \%$ hit the detector. The best signal could be obtained 


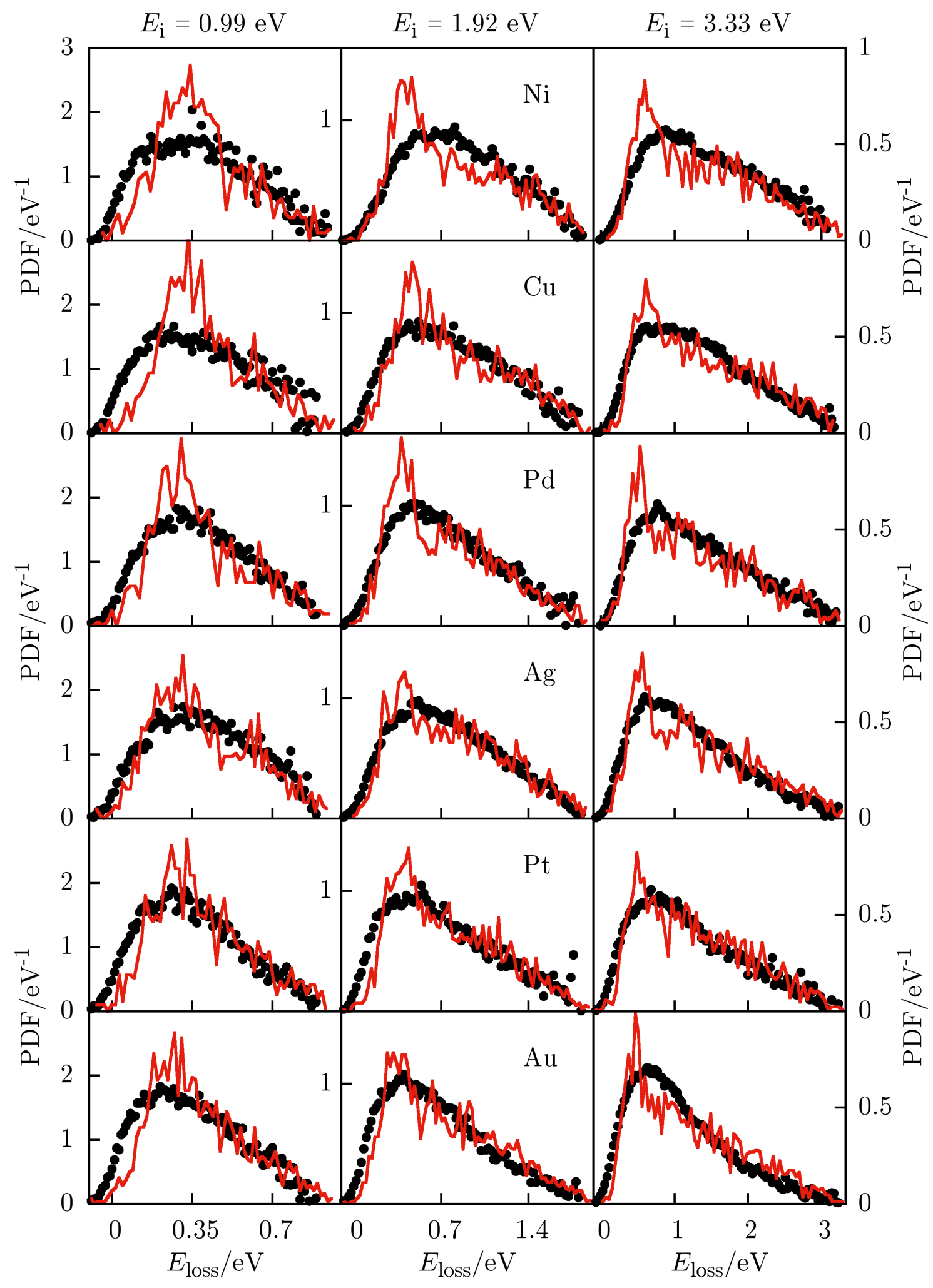

Experiment •

Theory —

Figure 30: Comparison between experimentally measured and calculated specular energy loss spectra of $\mathrm{H}$ atoms scattering from six different metal (111) surfaces at room temperature. The various metals are stacked vertically and enumerated in the central panels. Three incidence energies are displayed side by side. The projectiles are launched along the $[10 \overline{1}]$ direction and $\theta_{\mathrm{i}}=\theta_{\mathrm{f}}=45^{\circ}$. 


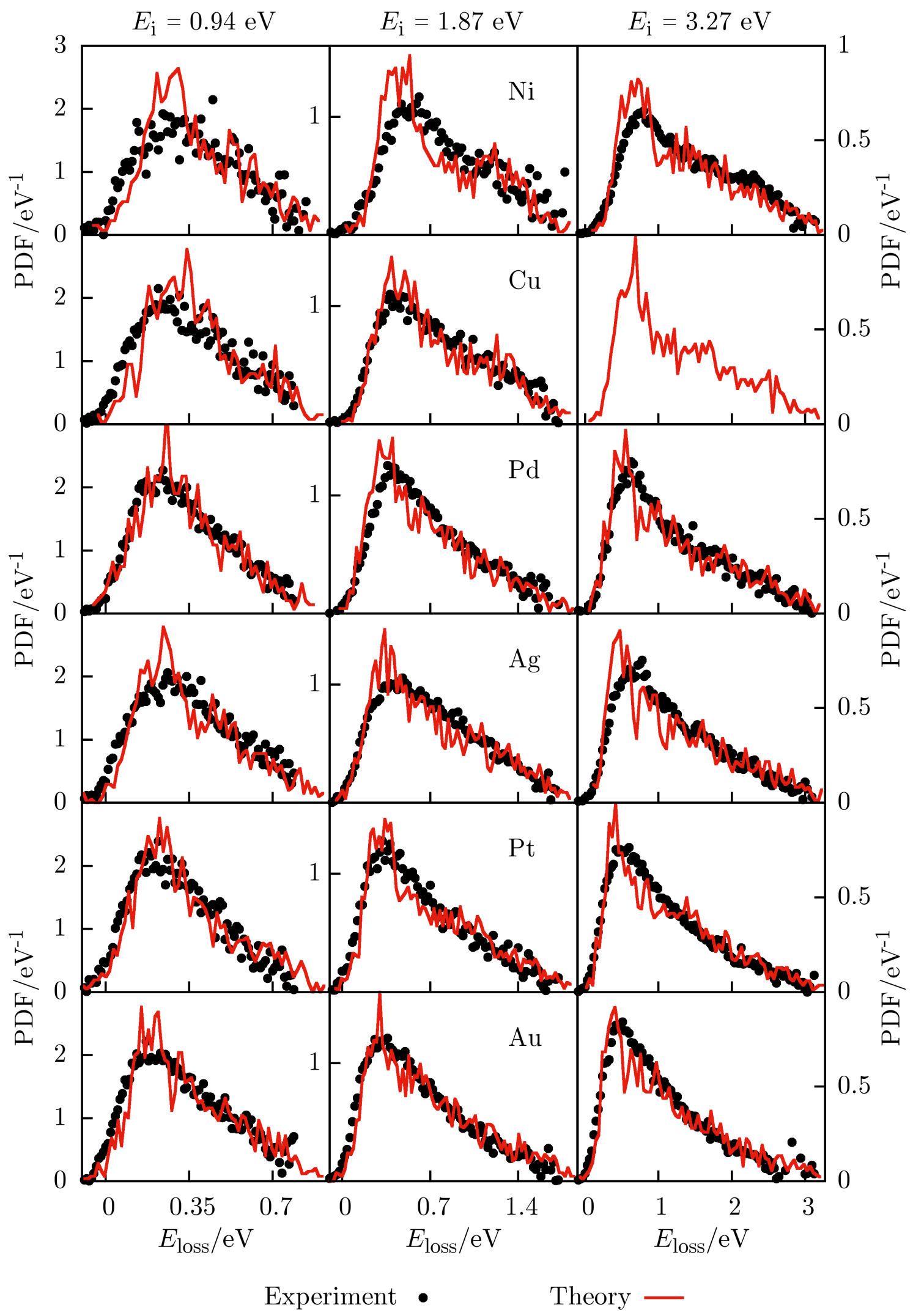

Figure 31: Same as Fig. 30, but with D atoms as the incident particle. 
from $\mathrm{D}$ atoms scattering off of $\mathrm{Pt}(111)$ at $E_{\mathrm{i}}=3.27 \mathrm{eV}$ which is based on 3841 detected particles. Here, $54.3 \%$ scattered of which $0.708 \%$ made it to the detector. The smaller sticking probability when launching high energy projectiles at a heavy metal clearly outweighs the fact that less scattered atoms reach the detector.

Overall, there is a good agreement between experiment and theory. The constant fractional energy loss predicted by theory and shown in Tab. 7 is supported by the experimental findings. Moreover, the intensity peak shifts to smaller energy losses as the mass of the surface increases. This fact and also its relative magnitude agrees very well with the predicted total energy loss spectra displayed in Fig. 22. Also, the high energy loss tail of the distributions is captured by the LDFA in all panels. This suggests that the electron density is the only decisive factor governing the observed high energy losses. The electron densities of the studied metals all lead to very similar friction coefficients which explains the similar behavior of light scattered atoms. There is no evidence for other possible influences like metal work function, magnetic properties and the density of states at the Fermi level.

As a general trend, the agreement systematically improves towards higher incidence energies. The better agreement at higher energies is due to small errors in the PES becoming negligible relative to the available energy. Since all the PESs were fitted using trajectory data where $E_{\mathrm{i}}=5 \mathrm{eV}$, their validity is ensured at all incidence energies used in experiment. Experimental data for the energy loss spectrum of D atoms scattering from $\mathrm{Cu}(111)$ at $E_{\mathrm{i}}=3.27 \mathrm{eV}$ was not available at the time of this writing. However, given that the curves all have similar shapes, one can expect the theoretical prediction to be fairly accurate. Further comparisons including angular distributions, a more sophisticated hard-cube model and an analytical expression for describing the sticking probability in dependence on incidence energy and polar angle have already been published in Ref. [3].

Another field of application is modeling chemicurrents with the parametrized fulldimensional PESs in combination with the LDFA. Since there is only a negligible isotope effect in the scattering experiments, why are chemicurrents induced by $\mathrm{H}$ atoms two to five times larger than than those induced by D atoms [176-179]? Since the full extent of this project has already been published [5], I only briefly describe the reason behind this phenomenon. From theory and experiment it is clear that the absence of an isotope effect in $\mathrm{H} / \mathrm{D}$ atom scattering is due to two compensating effects. $\mathrm{H}$ atoms lose more kinetic energy than $\mathrm{D}$ atoms to electron-hole pairs and it is the other way round for phononic excitation. As a result, the energy loss spectra become nearly indistinguishable. For chemicurrents, however, only the nonadiabatic 
excitations play a role. Hence, not only have $\mathrm{H}$ atoms a numbers advantage in term of excited ehps, but also the level of excitation is higher in case of $\mathrm{H}$ being the impinging particle.

The other reason which is immediately comprehensible, but not necessarily obvious, is that scattering and induction of chemicurrents are two entirely different things. In scattering experiments, only the particles that do not stick to the surface actually appear in the experimental data. We have seen that on average they lose 35 to $40 \%$ of their initial energy in the process. Particles that disappear into the bulk though lose energy until in thermal equilibrium with the surface. This is a much larger energy loss than the one experienced by scattered particles. It turns out, chemicurrents are caused mainly by projectiles that penetrate below the surface and eventually stick. This property is of course the same for $\mathrm{H}$ and D atoms, but it changes the ratio between phononic and nonadiabatic energy losses of $\mathrm{H}$ and $\mathrm{D}$ atoms in comparison with the ones shown in Fig. 24. The data in this figure is from scattering simulations in which the particles only experience a small number of bounces. One can imagine that dozens of collisions will further separate the H/D energy loss to phonons and ehps. These two reasons combined explain the large differences seen in experiment between larger H-induced chemicurrents and two to five times smaller ones induced by $\mathrm{D}$ atoms.

\subsection{PES Transferability}

Two extensions of EMT to other systems shall be presented in this section. The first one is to see how well an EMT PES that was trained with DFT data from one surface facet can reproduce interaction energies at a different surface cut. The other one addresses the applicability of EMT to the condensed phase of a rare gas.

\subsection{1 (100) and (110) Surfaces}

In principle, EMT can model any surface facet of an fcc metal. As long as each atom in the bulk has 12 nearest, 6 next-nearest and 24 next-next-nearest neighbors, nothing needs to be adjusted neither in the theory nor in the program. After all, the crystal is essentially the same, only the surface cut is different. Therefore, I was wondering whether an EMT PES parametrized with DFT data obtained from $\mathrm{H}$ at a (111) surface can reproduce interaction energies at different facets, as well. The only tested system is $\mathrm{H}$ on palladium. Its PES is neither particularly easy to describe, nor is it as corrugated as the $\mathrm{Pt}(111)$ surface for example. Furthermore, potential 
Figure 32: Top view of the (100) surface with six abbreviated high symmetry sites. Red spheres mark atoms in the surface layer and green symbolizes the first subsurface layer. The second subsurface layer (not shown) is the same as the top layer since the (100) surface is ABstacked.

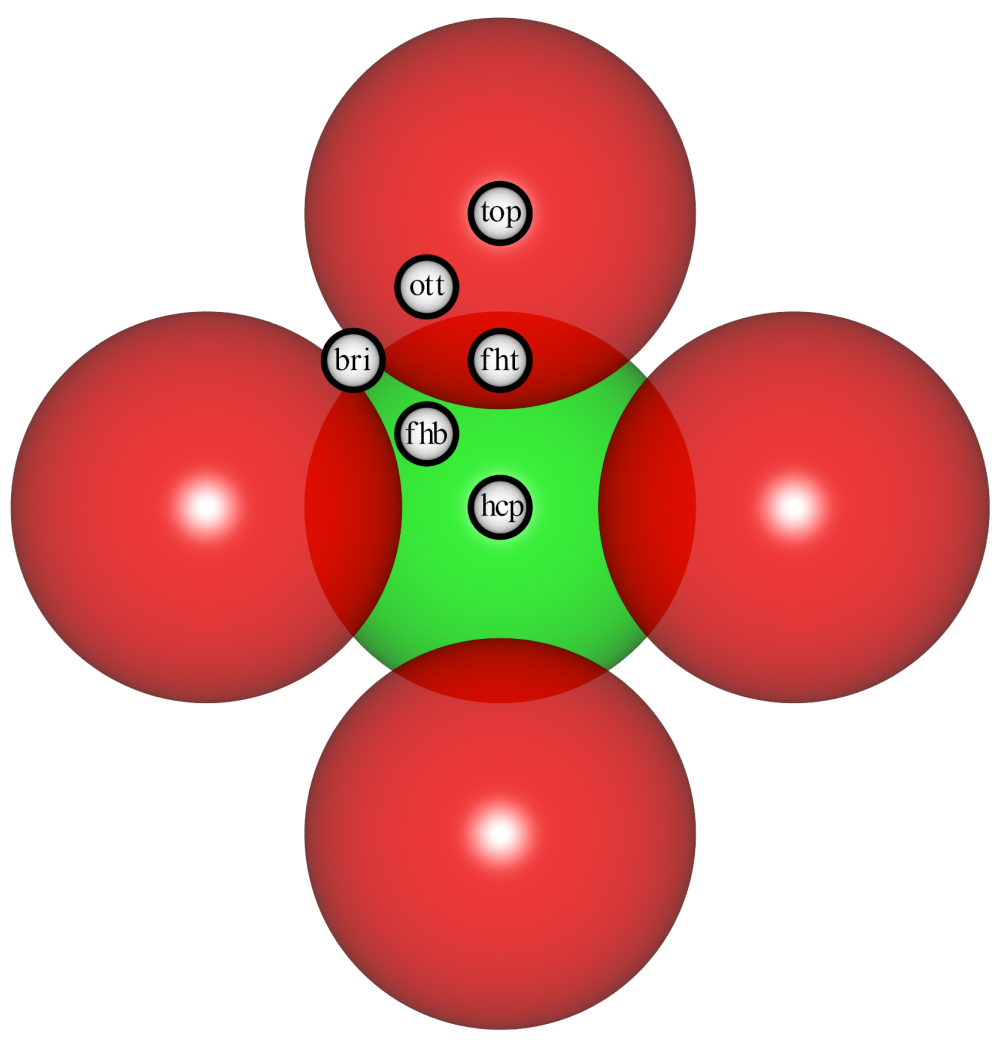

energy curves on the $\operatorname{Pd}(111)$ surface have already been discussed in section 4.1.2. After all, the metals all behave fairly similar. So it is safe to claim that if the interaction of $\mathrm{H}$ with a (100) and (110) Pd surface can be accurately described, the same is true for other fcc metals within acceptable bounds.

(100) The geometry of the (100) surface is shown in Fig. 32 together with the selected high symmetry sites and their abbreviations. It can be obtained by cutting the fcc unit cell such that the cut plane intersects only one axis. In contrast to the (111) surface, its unit cell is quadratic and consists of only two layers instead of three. This is also the reason why there are just four atomic layers simulating the surface in this paragraph, simply to reduce redundancies and speed up calculations. The number of surface sites is also reduced because of the simpler surface structure. In any case, six of them suffice to obtain a good estimate of the applicability of EMT to other surface facets.

To see how well the parametrized EMT PES performs on a previously unknown surface geometry, PECs from $a b$ initio DFT and EMT are compared in Fig. 33. It again fails to capture the full depth of the chemisorption well similar to the bridgesite in Fig. 14. Besides that, it reproduces DFT energies really well. Even though 


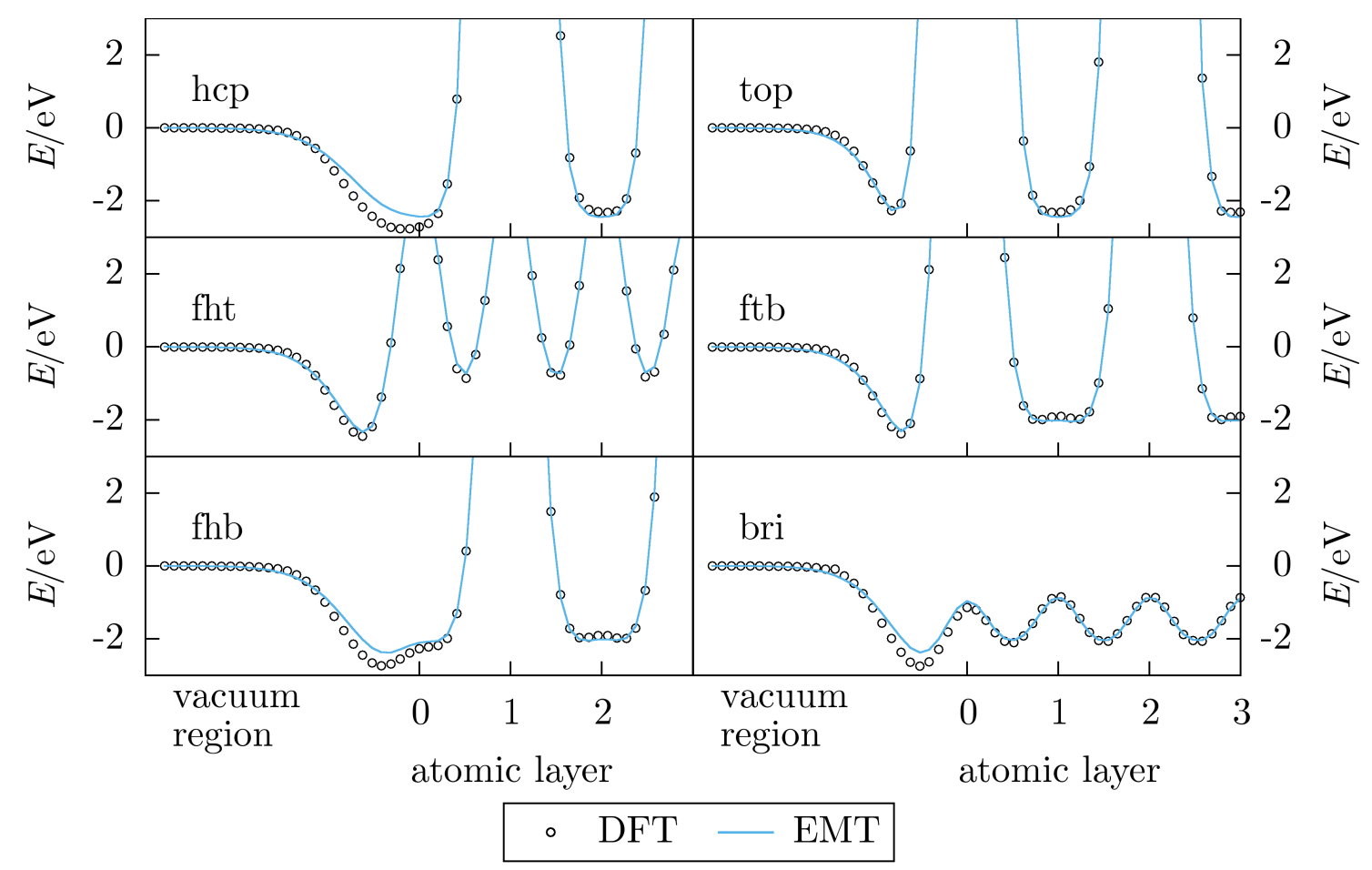

Figure 33: DFT and EMT potential energy curves of six high symmetry sites of a Pd(100) surface. The positions on the surface are shown in Fig. 32. Black circles mark ab initio reference energies and blue curves indicate energies of the parametrized EMT potential.

the surface structure is very different, the RMSE only amounts to $150 \mathrm{meV}$. I also confirmed that the EMT PES can predict energies from AIMD trajectories with identical accuracy.

(110) The other low-index surface of fcc crystals is called the (110) cut and its structure is shown in Fig. 34. It can be obtained by cutting the fcc unit cell such that the cut plane intersects two axes at $45^{\circ}$. Again, six symmetry sites are selected to probe the validity of the fitted EMT PES. These DFT single-point energy calculations provide another set of validation data and the comparison of PECs is presented in Fig. 35. The high potential energy peaks seen at the top and ffs panels are a bit wider compared to the top and hcp panels in Fig. 33. This is because the plots are scaled to the inter-layer distance of the surface atoms which is $\sqrt{2}$ times smaller in case of the (110) surface. Apart from that, the agreement is in line with all previous results obtained with the H-Pd EMT PES.

These calculations illustrate an important aspect of EMT in general and also of the PES parametrization procedure. I have shown that it is possible to reparametrize PESs on the basis of two data sets. One is comprised of fixed slab atom geometries 
Figure 34: Top view of the (110) surface with six abbreviated high symmetry sites. Red spheres mark atoms in the surface layer and green symbolizes the first subsurface layer. The second subsurface layer (not shown) is the same as the top layer since the (110) surface is also ABstacked.
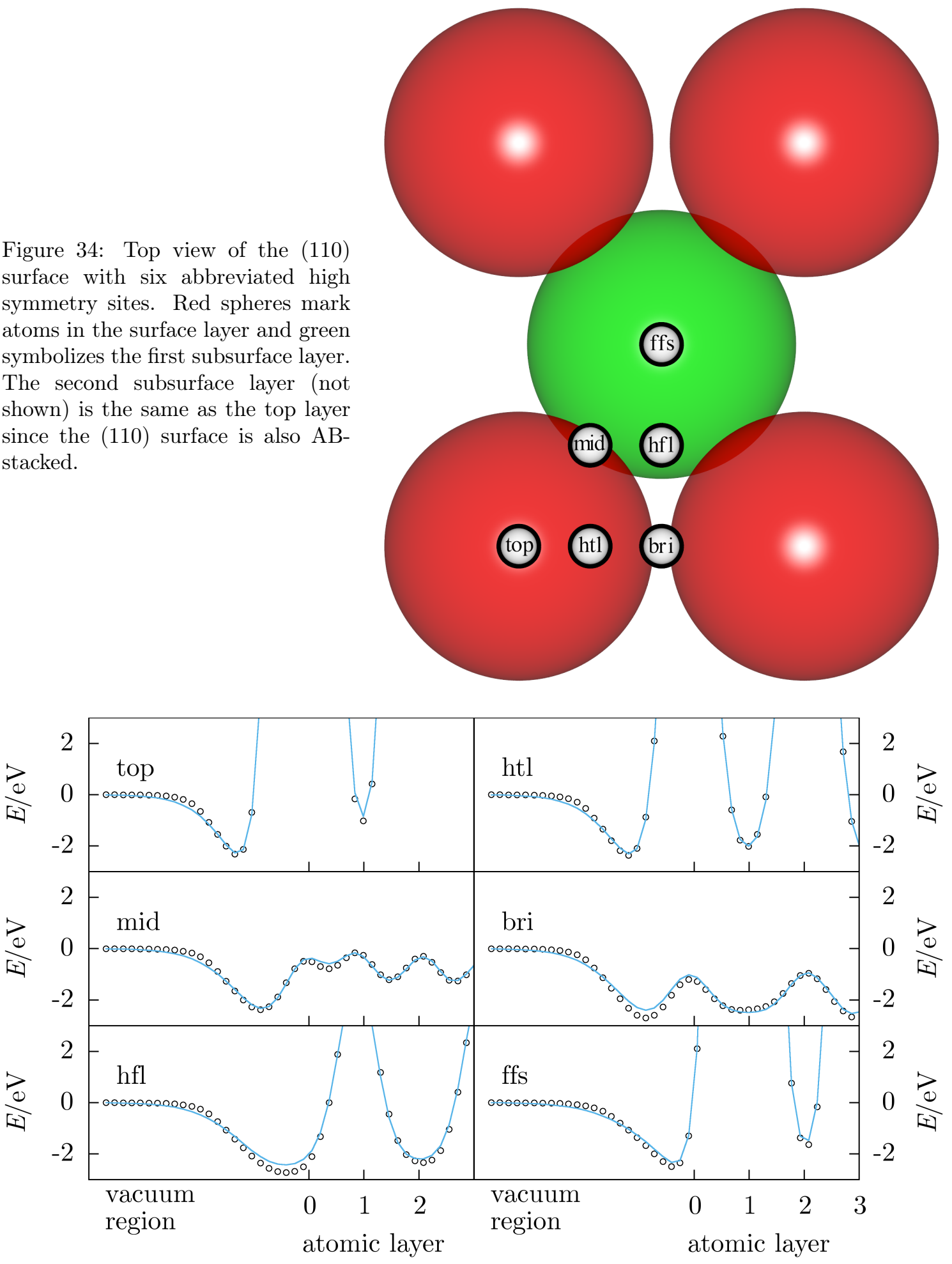

- DFT - EMT

Figure 35: DFT and EMT potential energy curves of six high symmetry sites of a $\mathrm{Pd}(110)$ surface. The positions on the surface are shown in Fig. 34. Black circles mark ab initio reference energies and blue curves indicate energies of the parametrized EMT potential. 
where the projectile is placed at selected positions above and inside the surface. The other one contains non-equilibrium geometries of this system extracted from AIMD trajectories. This setup is sufficient input for the genetic algorithm to find a robust parametrization that not only reproduces ab initio energies but also metal elastic stability criteria. The transferability of the PES was demonstrated by the reproduction of DFT energies of an $\mathrm{H}$ atom interacting with a different surface facet. A decrease in accuracy was observed neither for the frozen surface, nor for one in thermal equilibrium. If the best fits after GA optimization yield consistent scattering statistics as explained in section 4.1.2, and in consequence the selection of the final PES becomes arbitrary, it can be expected that the fit is transferable to different surface facets, as well.

For completeness, we have already briefly mentioned the validity of the $\mathrm{H}$ on $\mathrm{Pd}$ parametrization for different surface facets in Ref. [118].

\subsubsection{Xenon(111)}

In their 2015 paper, Bünermann et al. presented an energy loss distribution of $\mathrm{H}$ atoms scattering from a thick layer of xenon atoms [168]. They could cool the $\mathrm{Au}(111)$ surface in an ultra high vacuum chamber to $45 \mathrm{~K}$ and exposed it to gaseous $\mathrm{Xe}$ at $10^{-6}$ mbar for 5 minutes. Xenon easily freezes on the Au surface at this temperature [90] and forms a cubic close-packed structure [180]. The reason is that isotropic London dispersion forces are solely responsible for Xe to condensate at all. In consequence, its surface is the (111) facet since this cut minimizes the number of missing neighbors at the top layer [181]. The idea behind this experimental setup was to make measurements using a metal and an insulating surface within minutes of each other. Solid Xe could be easily released from the gold surface by heating.

In principle, EMT was designed to describe metals, impurities and alloys. It uses a sophisticated pair potential approach in which the surrounding directly influences the interaction strength of a pair of atoms. The question is, if EMT is flexible enough to describe a large variety of metals and their surface facets, is it also possible to use it for the interaction between a hydrogen atom and a noble gas surface? There are numerous examples in the literature [182-186] proving that already a simplistic Lennard-Jones (LJ) pair potential can accurately describe noble gas clusters and surfaces. The reason is simply that noble gas atoms are non-polar, neutral atoms which only interact via dispersive forces. The disadvantage is that all LJ potentials look the same in arbitrary units of length and energy. Also, the repulsive part is 


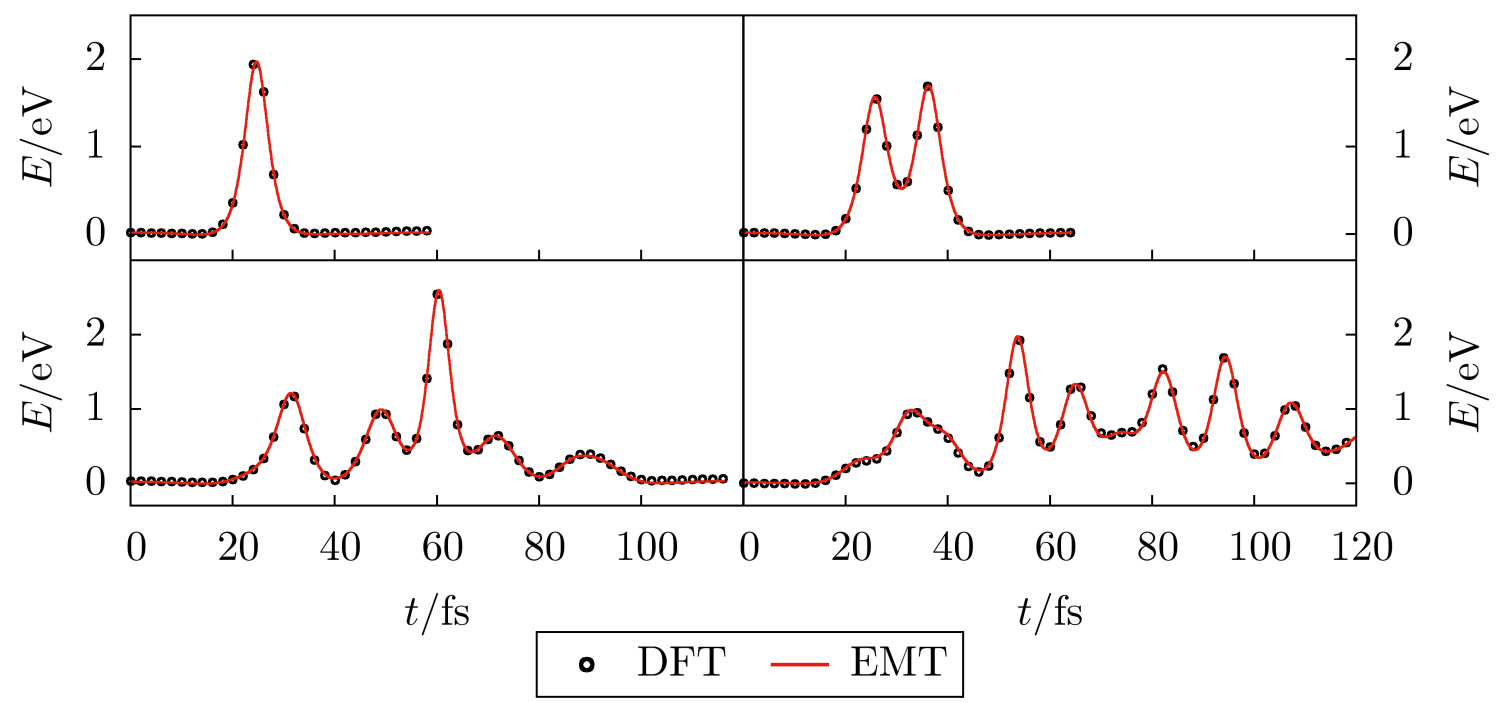

Figure 36: Comparison between the validation data AIMD energies (black open circles) and energies obtained with the optimized H on Xe EMT PES (red line). The DFT energies are only shown every 2 fs because one potential energy curve would not be visible if both were displayed by lines.

not physically motivated and its steepness is usually overestimated [187].

The EMT H on Xe(111) potential parametrization followed the same procedure as with the metals. A 3D DFT grid with $\mathrm{H}$ at high symmetry sites above and inside the surface was calculated and geometries from AIMD trajectories provided the nonequilibrium part of the training data set. Elastic constants of xenon at $45 \mathrm{~K}$ are of course difficult to obtain, therefore I decided to estimate them. We also know from the parametrization of $\mathrm{H}$ on metal surfaces that the elastic constants only play a minor role at the timescale of a scattering simulation. Especially because Xe atoms are two orders of magnitude heavier than the projectile, it cannot distort the lattice structure in any significant way. I only require that the elastic stability criteria be fulfilled, $B, C_{11}, C_{12}$ and $C_{44}$ be on the order of a few GPa and the Xe-Xe bonds be stronger than the $\mathrm{H}-\mathrm{Xe}$ bond.

Agreement with four AIMD trajectories from the validation data set is exemplarily displayed in Fig. 36. The black dots are ab initio energies and the red line is the predicted energy of the parametrized H-Xe EMT PES. In the trajectories the fourlayered Xe slab is at the experimental temperature of $45 \mathrm{~K}$. The projectile is provided with $2.76 \mathrm{eV}$ incidence energy, incidence polar angle is $30^{\circ}$ with the surface normal and it is launched along the $[10 \overline{1}]$ direction of the surface. Note that all involved energies, namely surface temperature and $E_{\mathrm{i}}$ of the $\mathrm{H}$ atom, are smaller than in the simulated $\mathrm{H}$ on metals AIMD trajectories. The reason for the change in setup is to model the experiment as closely as possible, not to generate an all purpose PES 
Table 8: Optimized EMT parameters for the description of $\mathrm{H}$ on Xe.

\begin{tabular}{cccccccc}
\hline & $\eta_{2} / \AA^{-1}$ & $n_{0} / \AA^{-3}$ & $E_{0} / \mathrm{eV}$ & $\lambda / \AA^{-1}$ & $V_{0} / \mathrm{eV}$ & $\kappa / \AA^{-1}$ & $s_{0} / \AA^{-1}$ \\
\hline $\mathrm{H}$ & 0.83760 & 0.19306 & -0.74340 & 2.53084 & 0.63797 & 1.640811 & 0.740725 \\
$\mathrm{Xe}$ & 2.18073 & 0.05603 & -0.16 & 1.76534 & 0.041711 & 2.49946 & 2.37018 \\
\hline
\end{tabular}

for the description of $\mathrm{H}$ on Xe. The obviously important van der Waals forces were taken into account on the level of DFT-D2 [188] with default parameters. Besides that, the methodology is the same as explained in section 3 .

Though the range of energies in the setup is smaller compared to previous parametrizations of $\mathrm{H}$ on metal PESs, it still spans over $2.5 \mathrm{eV}$. The red line passes through the center of nearly each reference data point. This can also be seen from the RMSE of this fit to both the DFT grid and non-equilibrium geometries. It only amounts to approximately $25 \mathrm{meV}$ which is remarkably small. In fact, if the way of representing the potential energy curves in Fig. 36 was the same as in Figs. 16 to 18 , one of the curves would not be visible because they overlap nearly perfectly. It seems like the absence of a real chemisorption well lets the optimization procedure focus more on all the repulsive parts.

The real test, however, is a comparison with the experiment mentioned at the beginning of this subsection. The Xe(111) slab in the simulation consists of six layers with 24 atoms per layer. The surface is at $45 \mathrm{~K}$ and the incident $\mathrm{H}$ atoms are provided with $2.76 \mathrm{eV}$. Polar incidence angle is $\theta_{\mathrm{i}}=45^{\circ}$ and the azimuthal direction is along $[10 \overline{1}]$. In this simulation, the detector has a diameter of $3.18^{\circ}$ solid angle just as in experiment. The resolution is still good enough because sticking is not common due to inherent electronic adiabaticity. Two sets of trajectory simulations were carried out: one uses the Lennard-Jones potential to characterize the interactions and the other one employs the parametrized EMT potential. The LJ potential parameters are $\varepsilon_{\mathrm{H}}=3.31 \mathrm{meV}, \sigma_{\mathrm{H}}=2.31 \AA, \varepsilon_{\mathrm{Xe}}=19.0 \mathrm{meV}, \sigma_{\mathrm{Xe}}=4.07 \AA$ from Refs. [183] and [189]. Parameter mixing was achieved by using the Lorentz-Berthelot mixing rules [190]. The optimized H on Xe EMT parameters are listed in Tab. 8.

The comparison of both sets to experimental data is presented in Fig. 37. Both panels show the same experimental data points. In the left panel they are displayed together with the specular energy loss spectrum obtained from a scattering simulation using the LJ potential. It shows a fairly small energy loss which can be expected and already matches the experiment quite well. Peak position and height 
can roughly be simulated. The right panel shows the comparison with the optimized $\mathrm{H}$ on Xe EMT PES. Here, peak position and intensity overlap nearly perfectly with the experimental data points. Also note that the distribution extends to higher energy losses which the LJ PES fails to capture. The reason is the lack of multibounce events when using LJ. Nearly no atoms visit subsurface sites which means they only collide one to two times with surface atoms before being scattered back. Also only $0.01 \%$ is absorbed by the surface at the end of the simulations. In EMT, the sticking probability is about $15 \%$ and $\mathrm{H}$ atoms can be found between all layers of the Xe-slab.

From a theoretical PES design perspective, one could argue that if LJ already yields an acceptable description, a more sophisticated version like EMT can only do better. However, EMT was designed on the basis of electron density arguments. In EMT the nuclei are supposed to bathe in a homogeneous electron gas which ensures atomic binding. It incorporates correction for $\mathrm{d}$-d orbital hybridizations and many more aspects which really do not apply to a Xe surface. Hence, the astonishing agreement only seems plausible in hindsight. To the best of my knowledge, this is the first prove that EMT is also capable of serving as a suitable PES for the interaction of $\mathrm{H}$ with a noble gas surface.

\subsection{Conclusion and Outlook}

I detailed in this section how to go about a reasonable procedure for parametrizing an EMT PES. Certainly there exist numerous ways to approach this problem. For this system at least, a mixed training data set of surface equilibrium and non-equilibrium structures has proven suitable. The genetic algorithm is then consistently able to produce reliable PESs. This approach is very robust and cannot only optimize the primary parameters, but also hyperparameters like the weight of AIMD data in the training set and the type of AIMD trajectory. The best resulting fits are all acceptable in terms of their deviation to the reference data. Often the elastic constants of the metal surface are in great agreement with literature values. But even in difficult cases like aluminum, it seems that the elastic moduli are not really significant for carrying out accurate scattering simulations. The reason is most probably that the projectile and surface atoms live on very different time scales. Hence, the impinging particle is either quickly scattered back so that the elastic moduli have no time to influence its trajectory or it is absorbed and does not appear in the energy loss spectra anyway. 


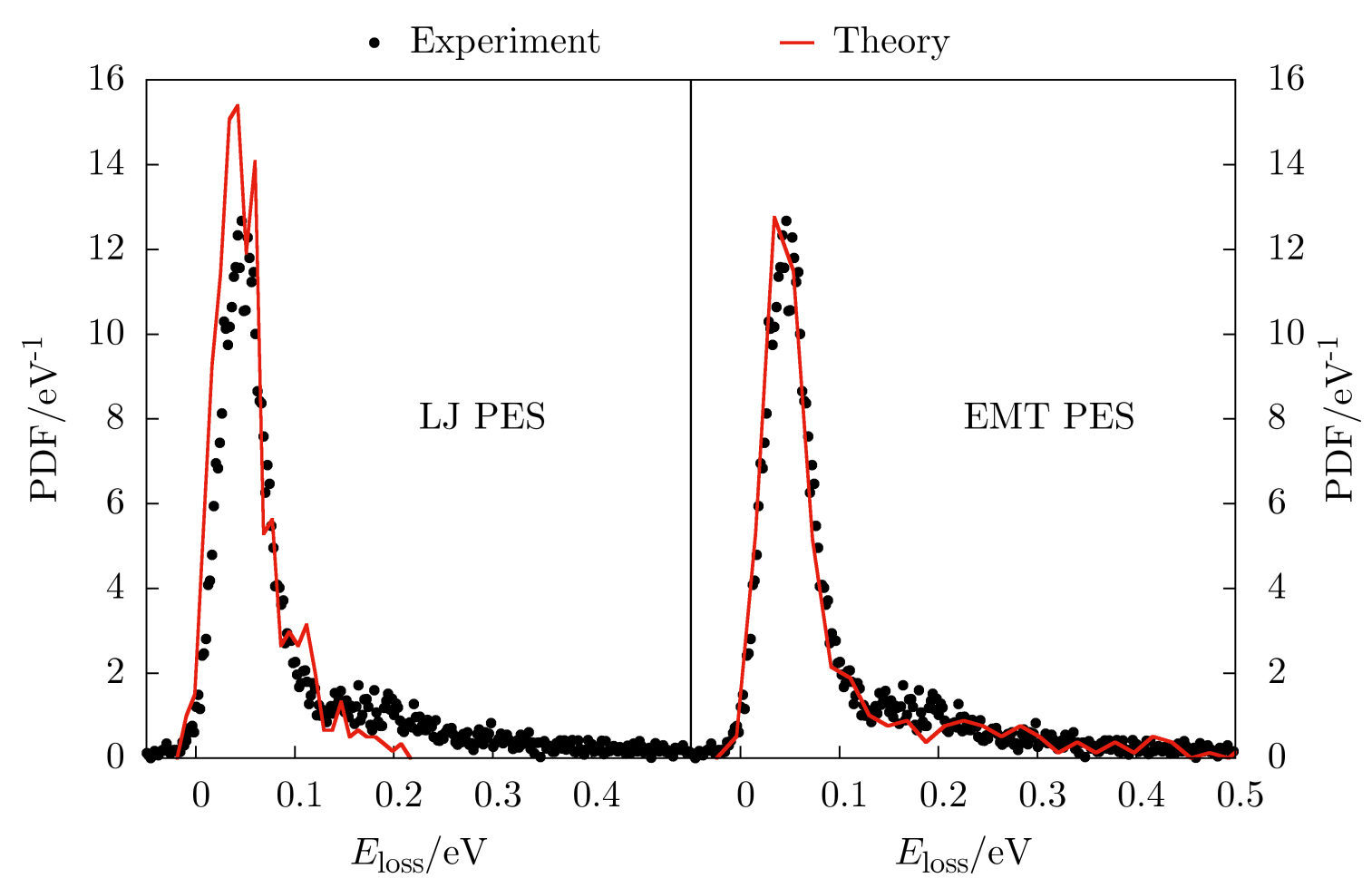

Figure 37: Specular energy loss spectra of H atoms scattering from a Xe(111) surface. Experimentally obtained data is shown as black dots and the red lines represent theoretical results. Incidence

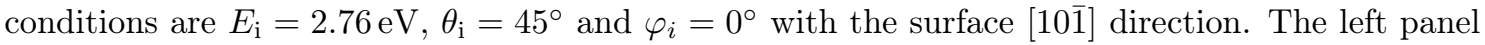
shows a comparison to the LJ potential with parameters from the literature [183, 189] and the right panel is based on the fitted EMT PES. The height of the panels shall highlight the heavy right tail of the experimentally obtained energy loss distribution which cannot be reproduced by LJ.

As far as energy loss spectra are concerned, the use of the LDFA has a huge impact. In the electronically adiabatic case, the order of the peaks simply follows the atomic number of the collision partner. Substitution with the deuterium isotope leads to peak broadening and a shift to higher energy losses. When electron-hole pair excitation is taken into account on the level of the LDFA, the results change dramatically. Both the peak and average energy loss increase, the spectra become much broader and an increased sticking probability can be observed. This behavior which has previously been observed for $\mathrm{H}$ scattering from $\mathrm{Au}(111)$ is also valid for many other fcc (post-) transition metals. Subsequent to these theoretical predictions, experiments were performed which validated the small but systematic changes in the spectra when moving from one period of the table of elements to the other. A decomposition into phononic and electronic energy loss reveals that the latter usually plays the larger role. This dissipation channel becomes all the more important the larger the mass of a surface atom. 
It turns out that EMT is a very versatile theory. A PES parametrized with geometries from one surface facet can seamlessly be used for the description of others. No new fit is necessary for this purpose. Furthermore, EMT can be applied to $\mathrm{H}$ atom scattering from a xenon surface. The agreement here is even superior to the description of $\mathrm{H}$ on metal surfaces, probably due to a higher regularity in the energy landscape.

In the future, scattering simulation could be performed from different surface facets and other crystal systems. The applicability to body-centered cubic (bcc) metals is currently being evaluated at the time of this writing [191]. Another route would be to complete the set of PESs for all fcc metals which still lacks Ca, Sr, Ir, $\mathrm{Pb}$, and $\mathrm{Yb}$. From the experimental side, it would be interesting to see results of $\mathrm{H}$ and $\mathrm{D}$ atom scattering from an aluminum surface. In this system the masses of projectile and surface atoms are fairly similar, especially when D atoms are used. In the energy loss decomposition paragraph, the calculations suggest that this is the only system in which the phononic contribution exceeds the electronic one.

Recently, a vacuum ultra violet laser was used in the Bünermann group to create $\mathrm{H}$ atoms for scattering experiments [192]. These projectiles have energies around 6 to $7 \mathrm{eV}$ and their energy loss spectra turned out to be difficult to describe with the current PESs. The reason can only be speculated about at the moment, but it might be due to an additional violation of the Born-Oppenheimer Approximation (BOA). $\mathrm{H}$ atoms with an incidence energy of $7 \mathrm{eV}$ travel at $37,000 \frac{\mathrm{m}}{\mathrm{s}}$ which might be too fast for electrons to follow instantaneously. Another reason could be that the LDFA parameters are not suitable for such high energies, but rather focus on an accurate description of low-lying electronic states. It could also be that the tensorial nature of electronic friction comes into play here [193, 194], or that the equations describing weak atom-metal coupling are not applicable anymore [195]. Another problem is of course the PESs presented in this work have only been trained with projectiles having an incidence energy of $5 \mathrm{eV}$. Hence, there is no reason to believe in their accuracy at extrapolated points in configuration space.

Although quantum effects most likely do not play a role in scattering light projectiles from metal surfaces since the surface atoms are heavy and there exists no barrier to adsorption, it would be interesting to look for them anyway. At least for subsurface diffusion simulations, which are also feasible with the PESs and the MDT2 program, they do matter. Ring Polymer Molecular Dynamics (RPMD) would be a great method for this purpose, but unfortunately, a rigorous theory that interfaces it with the LDFA does not exist yet. RPMD trajectory thermostatting can for 
example be accomplished with a Langevin thermostat, so there will eventually be a way to incorporate the friction coefficient. This would be a very interesting and challenging project from both the theoretical MD and software design perspective. 



\section{$5 \mathrm{H}$ on Graphene}

Carbon is an element that can form chemical bonds in a variety of different geometries. Diamond was probably known as early as $400 \mathrm{BC}$ and graphite since the $16^{\text {th }}$ century. The different hybridizations play an important role in today's commodity chemicals of which the simplest ones are acetylene (sp-carbon), ethylene ( $\mathrm{sp}^{2}$-carbon) and methane ( $\mathrm{sp}^{3}$-carbon). In 1985, fullerenes were added to the list of known carbon allotropes [196] and six years later, carbon nanotubes could first be synthesized in the laboratory [197]. At this point there were zero, one and three dimensional pure carbon materials available, but a 2D carbon sheet could only be realized in computer simulations at that time. As recently as 2004 Novoselov et al. were able to produce a single layer of carbon atoms by mechanical exfoliation which they called graphene [198]. A lot of research was conducted since then to understand and model its remarkable properties. These include but are not limited to a huge specific surface area [199], a carrier density over 1,000 times larger than copper [200], a resistivity at room temperature smaller than silver [201], and an exceptionally large thermal conductivity [202] and tensile strength [203]. For completeness, graphene is not the only two dimensional material. There also exists BN, $\mathrm{MoS}_{2}, \mathrm{NbSe}_{2}$ and $\mathrm{Bi}_{2} \mathrm{Sr}_{2} \mathrm{CaCu}_{2} \mathrm{O}_{\mathrm{x}}$ [204], whose interaction with $\mathrm{H}$ atoms might well become the topic of other projects.

Besides the extraordinary mechanical and electrical properties graphene is interesting for a variety of other reasons. Astrochemists are interested in this material because it might explain the large abundance of $\mathrm{H}_{2}$ in interstellar space. Two hydrogen atoms cannot simply collide to form a stable chemical bond since there is no way of dissipating the excess energy. Therefore, it requires a third collision partner which is imagined to be some carbonaceous material. The reaction then proceeds via the Eley-Rideal mechanism. One prerequisite though is that one $\mathrm{H}$ atom has already chemisorbed on the surface. The problem that arises is caused by the fact that the barrier to adsorption is approximately $200 \mathrm{meV}$ which corresponds to a temperature of $2300 \mathrm{~K}$. But the interstellar medium is much colder than this threshold. It is therefore still an active area of research how this initial adsorption can take place. [205]

Another interesting application of graphene-like materials is wall coating of thermonuclear fusion reactors. Many of these tokamaks are coated with a carbonaceous material. It was chosen because the hot plasma inside the reactor cannot be precisely confined by the magnetic fields at fusion temperatures. When at earlier times 
a heavy metal coating was used, hot plasma hitting the wall of the fusion chamber caused evaporation of metal atoms. Due to their large mass in comparison to the fusing hydrogen isotopes, the reaction is cooled down which impairs power output. The problem with carbon-based coatings which also partially applies to metal-based ones is that they possibly absorb some of the radioactive material. This was found to be the case in the Tore Supra in Cadarache, France, where a retention rate of $2.5 \cdot 10^{20} \mathrm{D}$ atoms per second was measured [206]. After a six minute plasma discharge, the $\mathrm{D}$ atom concentration in the deposited carbon layers was determined to be between $10^{17}$ and $10^{18} \mathrm{D} / \mathrm{cm}^{2}$ depending on surface temperature during discharge.

A different study came to the conclusion that the carbon-lined walls seems to endlessly absorb D atoms at least during this six minute period [207]. They state that in absolute numbers, between $50 \%$ and $60 \%$ of the injected particles are absorbed by the carbon layers. Both groups concluded that there must be an additional D atom acceptor somewhere in the reaction vessel that requires identification. These tests using Deuterium are important to see how radioactive tritium would behave in an actual $\mathrm{D} / \mathrm{T}$ fusion. Tritium retention has to be closely monitored first for safety reasons, but also for a possible economic success of the tokamak [208]. Understanding the mechanisms by which $\mathrm{H}, \mathrm{D}$ and $\mathrm{T}$ atoms interact with graphene would be a first step towards improvements of these carbonaceous claddings.

Theory nowadays plays an equally important role in this field. One future application in which $\mathrm{H}$ or $\mathrm{H}_{2}$ adsorption on graphitic materials is desired is storage purposes. $\mathrm{H}_{2}$ has the highest chemical energy to mass ratio which makes it suitable as fuel. Storing it under high pressure raises not only safety concerns, but also requires a lot of energy. It would be preferable to have a material which can uptake and release hydrogen like a sponge. Several graphitic compounds can be considered for this purpose and lots of calculations have been performed over the past 20 years. Carbon nanotubes have been investigated and were found to have a maximum $\mathrm{H} / \mathrm{C}$ mass ratio of $0.05-10 \%$ wt. Temperature and pressure strongly influence the amount of adsorbed hydrogen as does doping with alkali or transition metals. On graphene, the amount was calculated to be in the range of $0.2-4 \% \mathrm{wt}$, also depending on the conditions during adsorption. The superiority of nanotubes is attributed to the already strained structure which increases reactivity. All of these studies and many more are summarized in Refs. [209-211] which have also been used as sources for this paragraph. 


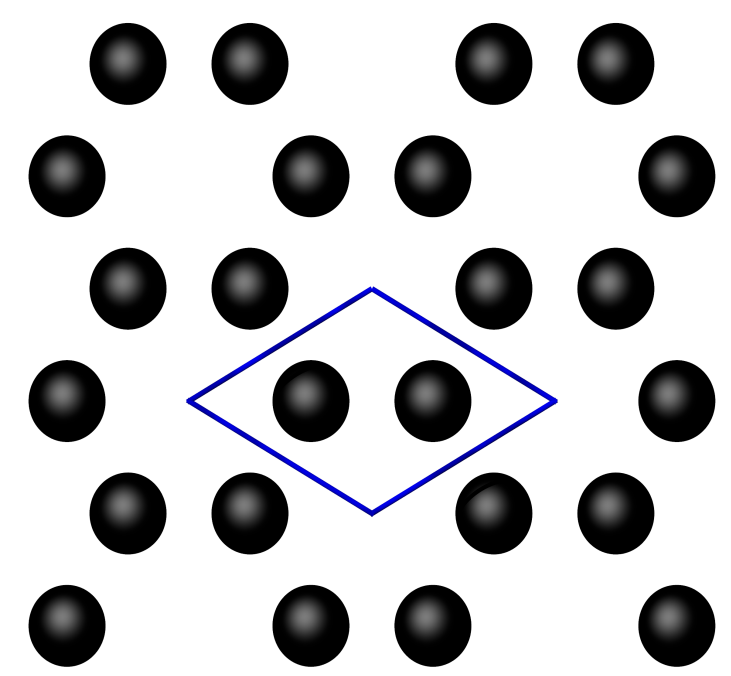

Figure 38: Black spheres indicate carbon atom positions in a graphene surface. This arrangement of 24 atoms is used in all scattering simulations from free-standing graphene. The blue diamond marks the primitive cell.

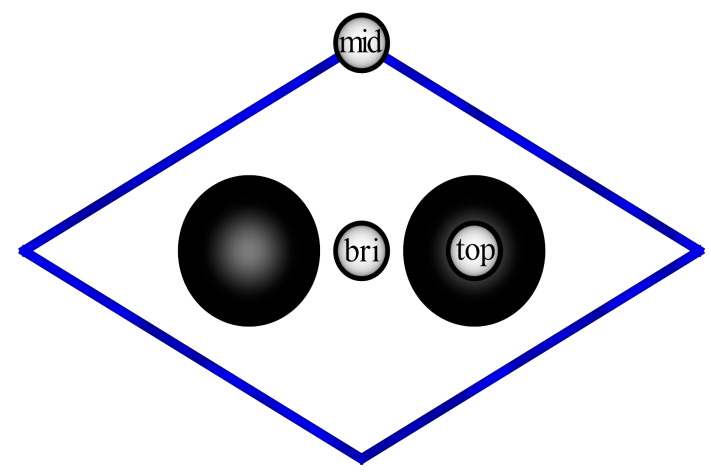

Figure 39: Primitive cell of free-standing graphene. Three high-symmetry sites are indicated by top, bri and mid labels. Note that in total there is one mid-, two top- and three bri-sites per primitive cell.

\subsection{REBO PES}

A Potential Energy Surface (PES) like Effective Medium Theory (EMT) is not able to describe the interaction between an $\mathrm{H}$ atom and a graphene surface. The nature of bonding, let alone different hybridizations and directional attractions, is fundamentally different from metallic systems. Therefore, a different PES needs to be employed for simulating $\mathrm{H}$ atom scattering off of graphene. In this regard, the motivation to use a full-dimensional PES for this purpose is the same as in the $\mathrm{H}$ on metals systems: $a b$ initio methods are computationally too demanding. The Reactive Empirical Bond Order (REBO) PES plays a crucial role in this chapter about an $\mathrm{H}$ atom interacting with an extended graphene surface. It shall be explained why REBO became the PES of choice for the description of this system and why a reparametrization was absolutely essential.

\subsubsection{PES Screening}

The literature offers several options for the description of $\mathrm{C}-\mathrm{H}$ and $\mathrm{C}-\mathrm{C}$ bonds. Some of them shall be briefly presented and discussed in the following paragraphs. A suitable PES must meet several requirements. First and foremost, it needs to be a reactive force field. Bond formation and breaking must be included in the formalism. It needs to provide directional forces which necessitates the use of bond 
angles, dihedrals and improper torsions. Moreover, the treatment of a carbon atom bond order of $\frac{4}{3}$ in graphene must be possible. Hence, it must not be mistaken as neither a single bond, nor a regular conjugated system in which each carbon atom has a bond order of 1.5 .

The Lennard-Jones (LJ) potential can be quickly labeled as unsuitable for obvious reasons. It can be applied to the interaction of molecular hydrogen with graphene [212, 213], but not to a single $\mathrm{H}$ atom. The Tersoff potential [65] was a revolution at the time of its publication in 1988. Many ideas were adopted in later force fields. For this application, however, it is not the best choice simply because it lacks four-body interactions. Additionally, de Tomas et al. showed that it does not even predict graphitization of amorphous carbon under simulated annealing conditions [214].

COMPASS [215] seems like a sophisticated potential for the description of condensed phase matter, but not so much for reactivity involving radical species. The UFF approach [216] is a holistic treatment of the entire periodic table of elements. It seems too general for the system of interest in this section. The ReaxFF [217] method seems more promising, especially because the title of the introductory paper reads A Reactive Force Field for Hydrocarbons. The same focus on carbon and hydrogen is present in the $2^{\text {nd }}$ generation REBO potential which eventually became the PES of choice. Nouranian et al. tested REBO and ReaxFF on small organic molecules and in principle found that both are very well designed, and both occasionally exhibit soft spots [218]. As an example, they found in a different and very extensive study [219] that REBO is better at describing alkane atomization energies, but ReaxFF has an edge at predicting radical atomization energies.

In the recent years and with the ever increasing interest in graphene and other $2 \mathrm{D}$ materials, REBO simply seems to be more visible in the literature while ReaxFF focuses more on the incorporation of oxygen, chalcogenides and metals into its formalism. I am convinced that ReaxFF would have also been a sound choice, but I decided to use REBO instead. REBO is superior in one key aspect: according to Ref. [214], it is about 60 times faster per simulated $\mu$ s, per atom and per timestep than ReaxFF. Since the energy evaluation with both PESs scales with $N^{4}$ with $N$ being the number of atoms and without further approximations, it is clearly advantageous to use REBO. In the end a potentially slightly higher accuracy in the dynamics does not outweigh a guaranteed higher precision due to better statistics.

For the same reason the adaptive intermolecular REBO PES [220] was disregarded. Although it contains dispersive terms which are relevant for the $\mathrm{H}$ on graphene system, it changes the scaling to $N^{6}$ [221]. In terms of the experiments 


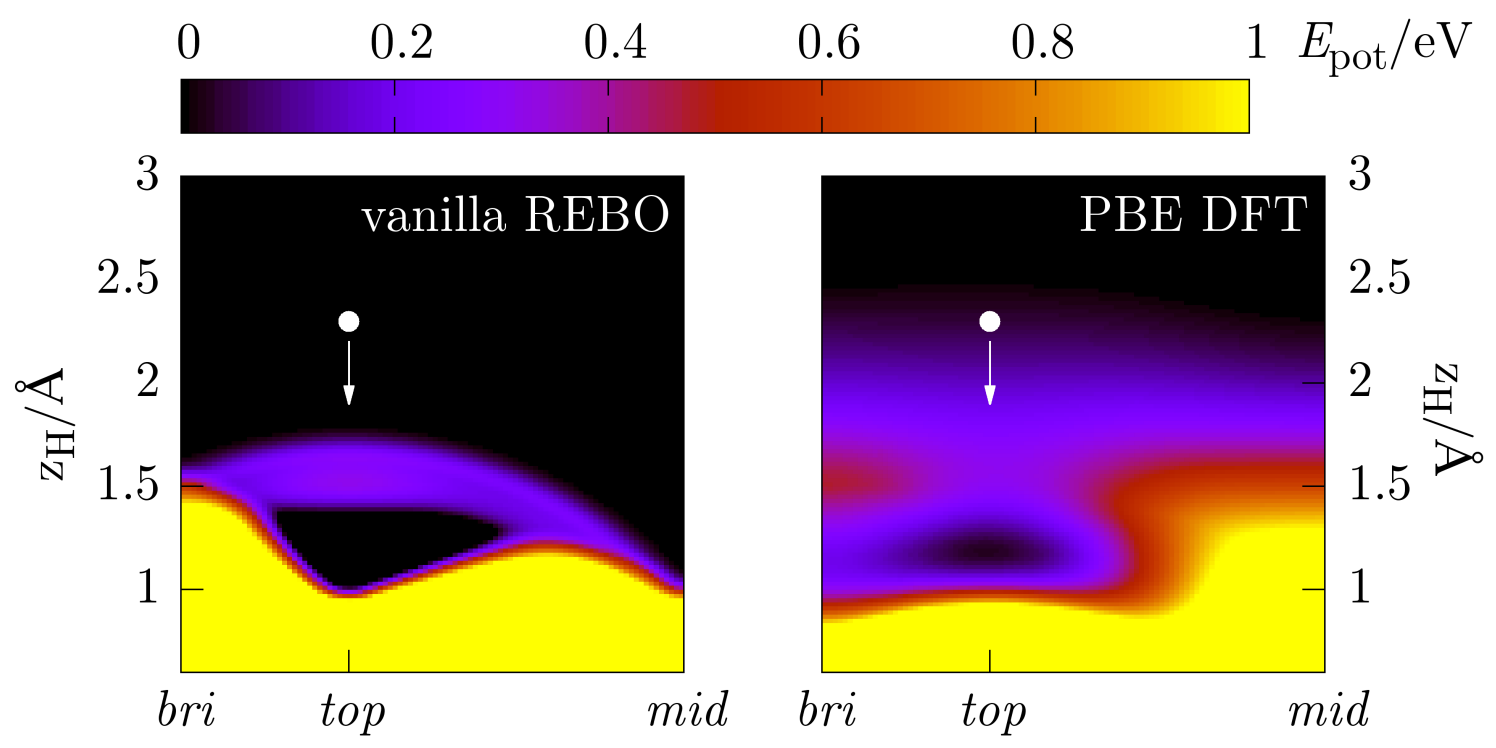

Figure 40: 2D cut through the PES of $\mathrm{H}$ on graphene. The $x$-axis runs from bri- via top- to the mid-site and the $y$-axis is parallel to the surface normal. The position of these sites on the surface is depicted in Fig. 39 and the potential energy is shown as the contour dimension. The white sphere symbolizes an $\mathrm{H}$ atom above the adsorption site and the arrow points directly towards it. The panels underline the vast differences between the original REBO parametrization and $a b$ initio DFT.

done in this group, the smallest $\mathrm{H}$ atom incidence energy is about $1 \mathrm{eV}$. Compared to the physisorption well depth of $\mathrm{H}$ on graphene of approximately $0.04 \mathrm{eV}$, and also respecting the typical Density Functional Theory (DFT) Generalized Gradient Approximation (GGA) error of roughly $0.2 \mathrm{eV}$, physisorption can be neglected. Note that the physisorption well depth which is nearly equal at all surface sites is confirmed both in experiment [222] and on the MP2 level of theory [223]. For physisorption studies the REBO potential is consequently inapplicable, but if real chemical accuracy is required, Molecular Dynamics (MD) potentials are not the right choice anyway.

The theory behind REBO can be found in section 2.3.2 and I implemented it into the MDT2 program to use it for surface scattering simulations. Except for minor errors ${ }^{1}$ all equations and splines can be found in the original publication [69] together with the default parametrization. To assess the quality of this PES, I created a potential energy heat map outlining the $\mathrm{H}$ atom adsorption channel in two dimensions. This plot shown in the left panel of Fig. 40 demonstrates the design of REBO. When an $\mathrm{H}$ atom approaches the top-site from right above it first encounters

\footnotetext{
${ }^{1}$ The arguments of $P_{i j}$ in Eq. 8 are in the wrong order if Tab. 8 which contains the corresponding spline coefficients is taken as the reference. The cutoff function in Eq. 20 is missing a factor of $\pi$ in the argument of the cosine.
} 
a barrier at $z=1.6 \AA$. This barrier to adsorption, which is not present in any $\mathrm{H}$ on metals system, exists due to re-hybridization of the carbon atom below. The electron cloud needs to reorganize, the bond order with neighboring $\mathrm{C}$ atoms needs to change and also the geometry has to adapt from trigonal planar to tetrahedral. Further towards the surface the $\mathrm{H}$ atom finds the attractive binding well at $z=1.2 \AA$ and if it continues its approach, it will climb up the repulsive wall.

This picture looks fairly reasonable and contains all features that one would expect from this system. To see how close the vanilla REBO PES resembles a higher accuracy method, I calculated a set of single-point energies using spin-polarized DFT with the Perdew, Burke, and Ernzerhof (PBE) functional. The same geometries were used, the plane wave cutoff was $400 \mathrm{eV}$, van der Waals interaction was taken into account on the level of DFT-D2, partial occupancies in each orbital were calculated with the tetrahedron method with Blöchl corrections [224], and a $\Gamma$-centered 8x8x1 $k$-point mesh was employed. The result is shown in the right panel of Fig. 40. It becomes immediately clear that the default REBO parametrization is wrong about several aspects about this cut through the PES.

First, the interaction starts way too close to the surface. In consequence, the surface would notice an approaching projectile just right at the barrier. The reaction to re-hybridize would be delayed which, due to reasons of inertia, is impossible to compensate at later stages of a collision event. Next, standard REBO predicts an attractive chemisorption well even without carbon atom puckering. Apparently, the carbon atom can stay in the surface plane and the $\mathrm{H}$ atom can still attach while releasing $0.6 \mathrm{eV}$ of energy. Such a conformation is not even allowed to exist according to valence shell electron pair repulsion theory and this binding energy will turn out to be the $\mathrm{C}-\mathrm{H}$ bond strength in a completely relaxed structure. The last difference between the panels is the extensions of repulsive walls at the bri- and mid-sites. Vanilla REBO suggests the repulsive region reaches further out at the bridge-site while DFT predicts that it is the center of the six-membered ring.

\subsubsection{Reparametrization}

It becomes clear from Fig. 40 that using the REBO PES "as is" will be of no use for an accurate simulation of scattering trajectories. Hence, the way to approach this problem is to try and reparametrize the PES. In REBO following the suggestions of Stuart et al. [220] there are 13 parameters to describe each interaction type. Therefore, 26 are required to characterize a single $\mathrm{H}$ atom in contact with a graphene 


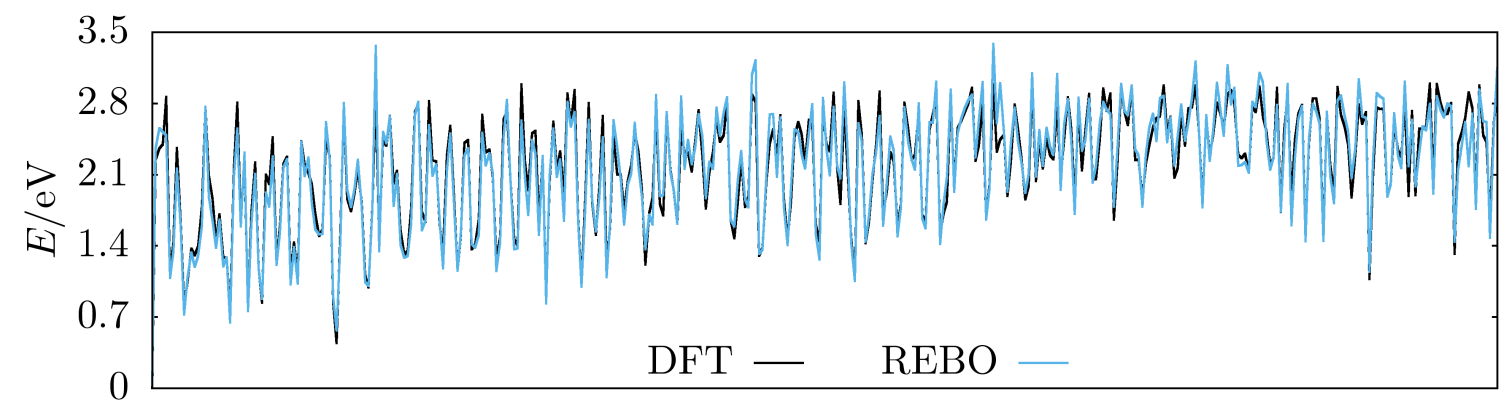

graphene configurations extracted during thermalization

Figure 41: Geometries visited in an AIMD graphene thermalization calculation from $0 \mathrm{~K}$ to $300 \mathrm{~K}$ were used for PES training purposes with an $\mathrm{H}$ atom randomly placed above the surface. Then, the energy of this configuration was calculated and is shown here as the black curve. The blue curve shows the best REBO PBE fit to this reference data. The trend towards higher energies reflects the additionally deposited thermal energy in the system as the thermalization proceeds. An interaction energy of approximately $3 \mathrm{eV}$ was taken as the upper limit for being included in the training data.

surface and 39 would be needed if two or more $\mathrm{H}$ atoms were present. Two valid reparametrizations resulting from different approaches were achieved in this work and both have their right to exist. The two parameter-optimizing procedures are described in the following paragraphs.

5.1.2.1 PBE Reference The first reference data set was created using plane wave DFT with the settings described at the end of section 5.1.1. In this system it turned out disadvantageous to create a grid-like structure serving as a backbone to the fit. The multi-body interactions simply limit the explorable configuration space. Choosing high symmetry sites and varying the H-surface distance only samples $\mathrm{C}$ $\mathrm{H}$ distances, C-C-H bond angles, and C-C-C-H dihedrals. The puckering of a $\mathrm{C}$ atom would be neglected in this case. And even if three dimensions for the carbon coordinates were included, the reaction of the neighboring carbon atoms would still remain unconsidered. Therefore, a different approach had to be taken.

In any case AIMD trajectories of $\mathrm{H}$ atoms scattering from graphene would be necessary at some point. Surface snapshots at finite temperature are an important prerequisite for this purpose. The advantage is that geometries visited during an equilibration procedure thoroughly sample configuration space without any manual assistance. If started from a relaxed geometry subject to subsequent heating, this process also covers the entire temperature range from $0 \mathrm{~K}$ to room temperature in this case. At any temperature the system tries to approach the Boltzmann distribution of positions and velocities, automatically weighting occurring geometries with 


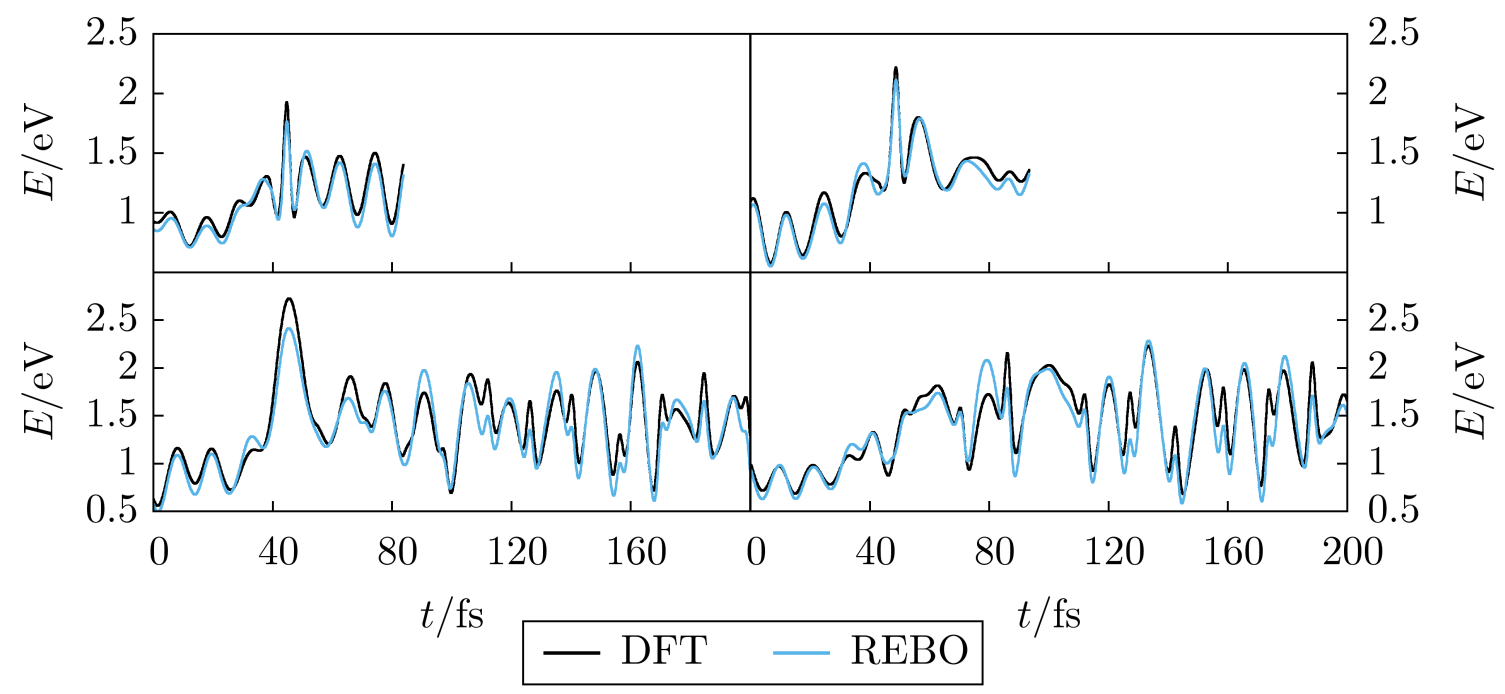

Figure 42: AIMD trajectories of $\mathrm{H}$ scattering from graphene were used as validation data for the fit. Ab initio energies are shown as black curves. Blue curves indicate energies predicted by the REBO parametrization. Single-bounce trajectories are depicted in the top row, while those demonstrating sticking events are shown in the lower two panels. The utilized parameters are the same as in Fig. 41 which is overall the best fit to PBE DFT energies.

their respective probability.

The training data set was then built in the following manner: an $\mathrm{H}$ atom was added at a random position to all geometries from the surface thermalization run. This effectively converts the time series of heavily redundant geometries into a set of a few thousand snapshots whose energetic correlation has mostly been removed. Then, a set of single-point energy calculations was run which determined the new energy of each system snapshot including the randomly placed $\mathrm{H}$ atom. These approximately 4,000 pairs of configurations and energies were subsequently used as training data. A sample of them is shown in Fig. 41 in combination with a fit to this data. To also incorporate structures of the adsorbed $\mathrm{H}$ atom with the $\mathrm{C}$ atom being $\mathrm{sp}^{3} \mathrm{I}$ used other geometries in which the four $\mathrm{C}$ atoms closest to the projectile were relaxed. In total the training set size amounted to close to 5,000 data points and the optimization was done with the trust-region nonlinear least squares algorithm which was also responsible in the GA for optimizing $\mathrm{H}$ on metals PESs. There were many more configurations available, but I selected only those with an interaction energy below $3 \mathrm{eV}$. Now this may seem small, however, the sophistication of the REBO PES cannot be stressed enough and too high energies during training prohibit important subtleties from being accurately described.

Validation data for the fits consisted of geometries extracted from AIMD trajectories. I chose to run 200 AIMD H atom scattering trajectories with the free-standing 
graphene surface at $300 \mathrm{~K}$. H atoms were provided with $1.92 \mathrm{eV}$ initial kinetic energy, the incidence polar angle was $52^{\circ}$, and azimuth was parallel to a C-C bond. In case the fitting procedure had not yielded any usable results there would have at least existed some data to compare with experiment. About 10,000 data points were used for validation and four AIMD trajectories together with the predicted REBO energies are presented in Fig. 42. This REBO-PBE fit has an Root Mean Square Error (RMSE) of $160 \mathrm{meV}$. The Potential Energy Curves (PECs) look very different from $\mathrm{H}$ scattering off of metals. Compared with Figs. 16 to 18 for example the profile of the single-bounce trajectories shows a much more oscillating behavior. The point of closest projectile-surface approach, clearly visible in the mentioned H-metal trajectories as a single peak, nearly gets lost in the top row of Fig. 42. Though it can still be discerned as the highest peak at around $45 \mathrm{fs}$, the carbon atom motion is clearly happening on the same time scale as the collision event. This insight already foreshadows a dramatically different projectile-surface interaction mechanism which will be elucidated in the following sections.

Unfortunately the Genetic Algorithm (GA) can not be used for fitting the REBO PES. The main reason is the higher computational demand of this potential. While optimizing the EMT PES, the GA produced about 60,000 fits a week. In the same time, not even one tenth of these fits finish using REBO and the training set described in the previous paragraph. Notwithstanding that the typical metal slab comprises 216 atoms and the free-standing graphene layer consists of just 24 atoms, it takes this much longer. Next, there exist no secondary fit criteria like elastic constants, C-H bond energy or sublimation enthalpy. This was actually the real strength of the GA to find fits that match those criteria while simultaneously reproducing $a b$ initio energies.

I usually set the initial parameters to those found in the original publication with some $10 \%$ to $20 \%$ random deviation. It turns out that in this system the resulting fit is strongly dependent on the input data set. In contrast to $\mathrm{H}$ on metals, where basically any AIMD trajectory suffices to probe non-equilibrium configurations, here the setup really matters. Things like whether the projectile interacts with just one or more carbon atoms, whether it sticks to the surface or hits the bri- or midsite massively change the results. This is why no AIMD trajectories were used for optimizing the parameters but only for validating them. There was a steep learning curve in what led to better and better fits, but still one needs multiple cuts through this highly complex PES to ensure a fit had really worked. Try and error was the method that finally produced a reasonable cut through the PES which is shown 


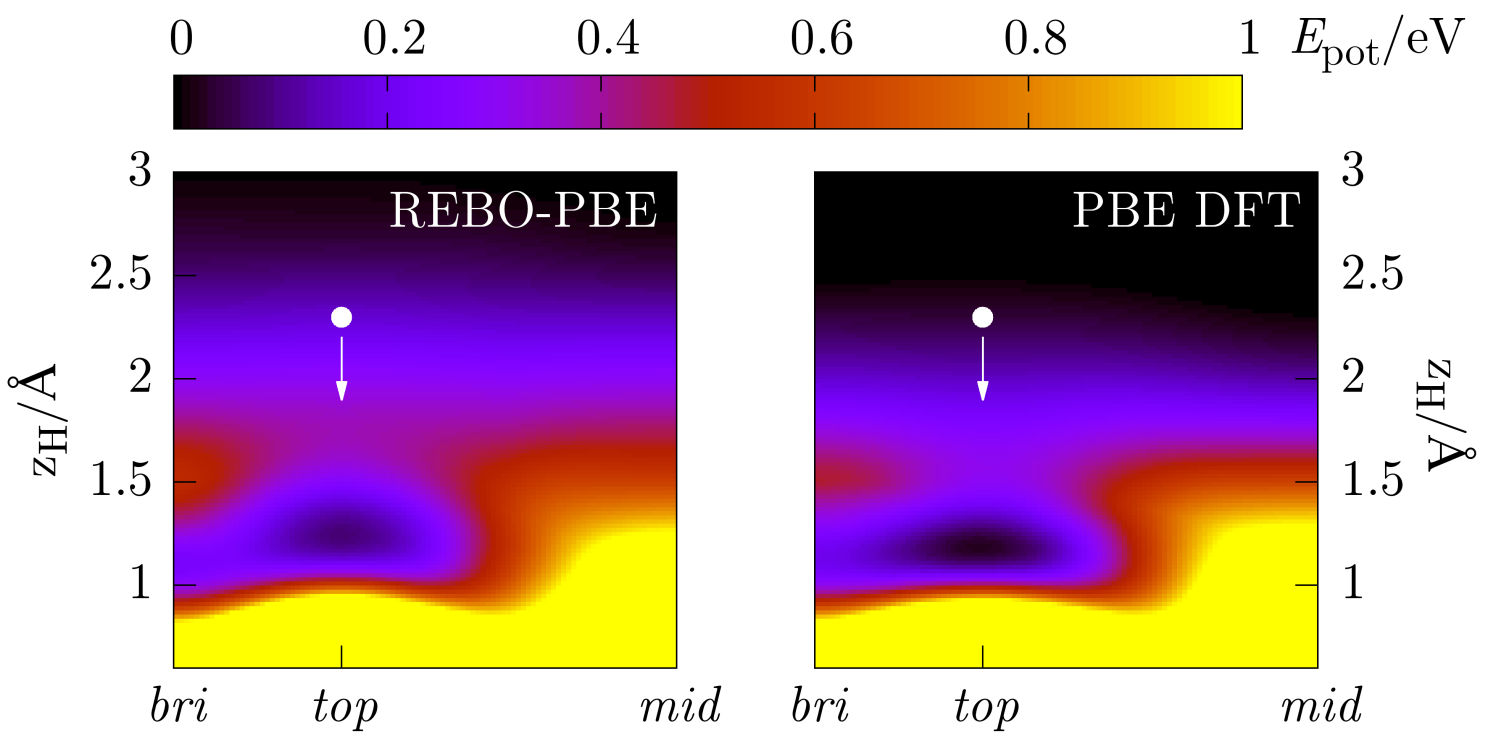

Figure 43: $2 \mathrm{D}$ cut through the PES of $\mathrm{H}$ on graphene. The $x$-axis runs from bri- via top- to the mid-site and the $y$-axis is parallel to the surface normal. The position of these sites on the surface is depicted in Fig. 39 and the potential energy is shown as the contour dimension. The white sphere symbolizes an $\mathrm{H}$ atom above the adsorption site and the arrow points directly towards it. This is the best fit to the PBE DFT reference data and is referred to as "REBO-PBE".

in Fig. 43 using the same scheme that was initially employed to demonstrate the deficiency of the vanilla REBO parametrization for scattering simulations.

This reparametrized REBO-PBE potential now has a better onset of the interaction region between 2 and $2.5 \AA$. It may even start a bit too far out, but I feel like this is necessary due to limited flexibility in the potential to reproduce the important barrier and adsorption well regions. At least I have never encountered a fit in which all three features were modeled satisfactorily. Hence, it was decided that the onset of the adsorption barrier is the least important one. Next, the minimum of the well without $\mathrm{C}$ atom puckering is close to $0 \mathrm{eV}$ which is another big improvement over the REBO vanilla parametrization. As the last thing to note the repulsive parts of this PES now agree very well with the picture we get from DFT.

5.1.2.2 EMFT Reference Since it could be shown that REBO can capture important aspects of the H-graphene interaction, a better DFT reference method would eventually lead to a more accurate MD potential. This assumption requires of course the deviation from $a b$ initio data be the same. PBE, however, is known to underestimate the barrier to $\mathrm{H}$ atom adsorption on graphene. Wang et al. calculated PECs of $\mathrm{H}$ atom adsorption on pyrene and coronene using various methods [225]. Though these are isolated graphene sheets consisting of four and seven annealed benzene 
rings, respectively, they found that barrier heights differ by no more than $30 \mathrm{meV}$. This finding is attributed by them to the fact that the transition state region is barely affected by system size. Their most accurate numbers using G2MS [226] to estimate ROCCSD(T) [227] energies with the cc-pVTZ basis set [228] amount to a barrier height of $390 \mathrm{meV}$ (pyrene) and $400 \mathrm{meV}$ (coronene). PBE on the other hand, predicts it to be $250 \mathrm{meV}$ (pyrene) and $240 \mathrm{meV}$ (coronene), or according to Ref. [229] only $200 \mathrm{meV}$. This agrees with my PBE DFT calculation shown in Fig. 43, which yields about $300 \mathrm{meV}$. But this value is an absolute upper bound since in contrast to the cited references, the surface was not allowed to relax in this calculation. However, the configuration of the surface does play a crucial role and a change of $100 \mathrm{meV}$ can easily be caused by limiting degrees of freedom (DOFs).

Wang et al. also note that calculations using unrestricted B3LYP [230] "are sufficiently accurate for the description of the chemisorption of a single hydrogen atom on a graphitic surface". Counterpoise corrected estimates using this level of theory give a barrier height of $330 \mathrm{meV}$ for $\mathrm{H}$ atom adsorption on pyrene and $340 \mathrm{meV}$ on coronene. The other theoretical basis for generating higher-than-PBE level reference data is called Embedded Mean-Field Theory (EMFT) [44, 231, 232]. With the help of this embedding scheme two DFT functionals can be used simultaneously to calculate the energy of a system. The idea is to describe the crucial part of the system like the reaction site using a computationally expensive functional with an extended basis set and parts further away with a cheap functional and a reduced basis set. With this approach, knowledge can be gained about the higher level of theory as if the entire system were using it, but at a reduced computational expense. This approach was taken to accumulate $a b$ initio reference data for fitting another parameter set of the REBO potential.

Using the knowledge that B3LYP is an appropriate functional for describing $\mathrm{H}$ on graphene it is used in combination with the cc-pVDZ basis set [228] to describe the high-level region. The low-level region is modeled by the Local Density Approximation (LDA) [30, 233] and the STO-3G basis set [234]. Calculations were performed using the entos software package [235] which implements standard Self-Consistent Field (SCF) procedures. The initial orbitals were guessed by a superposition of atomic densities and direct inversion of the iterative subspace accelerated the SCF cycles. Convergence was achieved when the maximum value of the orbital gradient was below $10^{-5}$ a.u.. Since this software package uses localized orbitals convergence tests were necessary to find a graphene flake size that is on the one hand computationally feasible and on the other hand gives converged energies. The same is true 
Figure 44: $\quad \mathrm{C}_{42} \mathrm{H}_{16}$ flake used to produce EMFT reference energies for fitting the REBO-EMFT potential. The central 16 carbon atoms (red) are modeled with B3LYP and the cc-pVDZ basis set, while the outer carbon atoms (black) and terminal $\mathrm{H}$ atoms (white) are treated on the level of LDA with a STO$3 \mathrm{G}$ basis set. In the AIMD EMFT trajectories, the $\mathrm{H}$ atom was always aimed at the central unit cell, to minimize the influence of the low-level region on the scattering dynamics and energies.

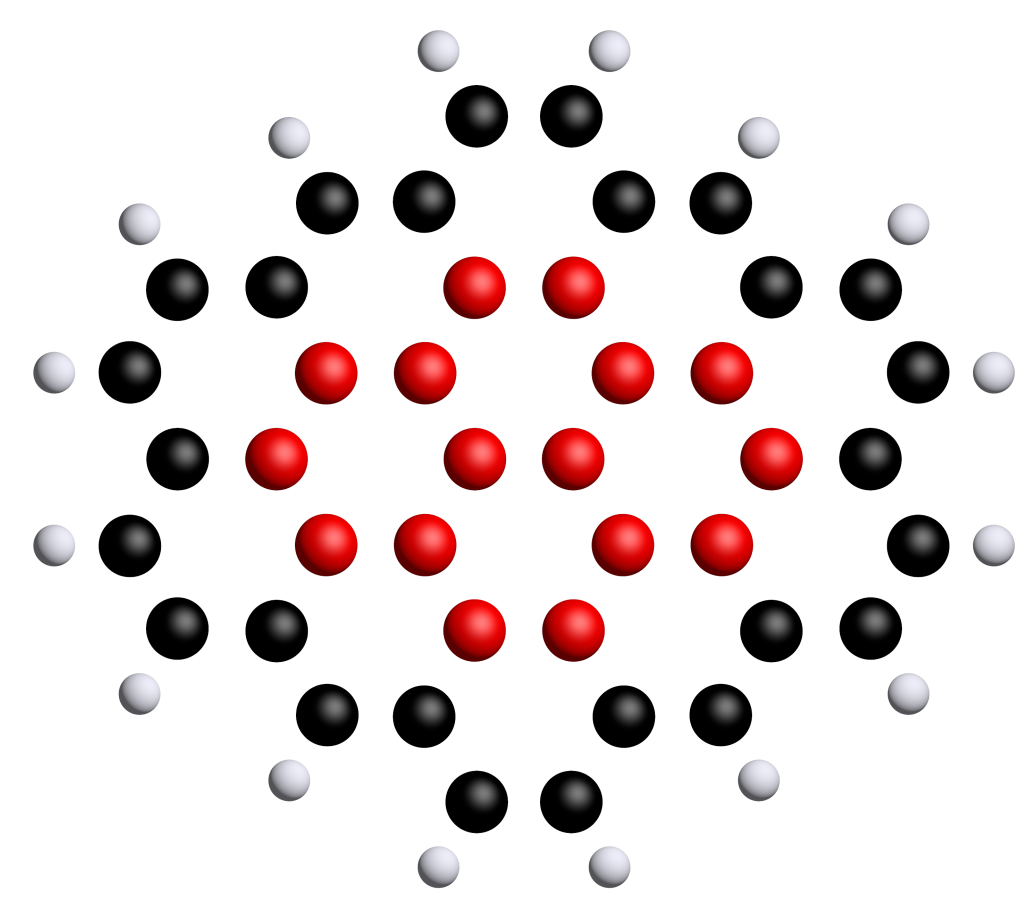

for the size of the high- and low-level regions.

It turns out that the $\mathrm{C}_{42} \mathrm{H}_{16}$ molecule with the central 16 carbon atoms being modeled with B3LYP fulfills both criteria. The molecule is shown in Fig. 44. By treating the outer carbon and terminal $\mathrm{H}$ atoms with the LDA, an eleven times speed-up could be achieved versus modeling the entire system at the high level. ${ }^{2}$ No van der Waals correction was applied, but REBO would not be able to model the physisorption well anyway. All EMFT calculations were performed by Feizhi Ding in the group of Tom F. Miller III. They generously provided me with data to reparametrize the REBO potential based on their higher level calculations. Some results shown in this work based on the REBO-EMFT parametrization have already been published in Ref. [6].

The route that was taken to fit the REBO-EMFT PES is different from the previous one, but in the end sampled configuration space in the same way. 400 AIMD trajectories were used as reference data. In 300 of them, the $\mathrm{H}$ atom was provided with $1.92 \mathrm{eV}$ incidence kinetic energy starting at $5 \AA$ above the surface. Its initial polar angle varied between $0^{\circ}$ and $50^{\circ}$ and the azimuthal angle was selected at random. The target position on the graphene flake was randomly located within the central unit cell that belongs to the high level region. The flake itself was also modeled by the $\mathrm{C}_{42} \mathrm{H}_{16}$ molecule which is still large enough to reproduce the

\footnotetext{
${ }^{2}$ Speed-up achieved in terms of wall-clock times using a NERSC Haswell computer with 32 cores
} and 128 GB DDR4 $2133 \mathrm{MHz}$. 
barrier height of $\mathrm{C}_{130} \mathrm{H}_{28}$ to $50 \mathrm{meV}$ accuracy on the B3LYP level. At the start of each trajectory a surface snapshot which had previously been thermalized to $300 \mathrm{~K}$ using the Andersen thermostat was chosen at random. In the remaining 100 AIMD trajectories, the $\mathrm{H}$ atom was launched towards the slab starting at $1.2 \AA$ with $2 \mathrm{eV}$ at an incidence polar angle of $30^{\circ}$. The rest of the setup was the same as before. This way the projectile did not influence the graphene geometry on its way towards the surface and it was also able to approach closer to the surface plane. From this collection of geometries 1200 were selected to serve in the training data set whose acceptance criteria are explained in the next paragraph together with the remaining training data.

For the fit I used 600 geometries with a C-H distance below $1.1 \AA$ and 600 where the $\mathrm{C}-\mathrm{H}$ distance is larger than $1.1 \AA$ from the set of trajectories. This value is approximately the equilibrium $\mathrm{C}-\mathrm{H}$ bond distance. Since the carbon atom that the $\mathrm{H}$ atom adsorbs to puckers out of the surface during binding, I made sure that the configurations were evenly distributed in the puckering dimension. In addition, I used 200 configurations from EMFT single point energy calculations that correspond to a graphene temperature of $20 \mathrm{~K}$ in which the $\mathrm{H}$ atom was placed between $0.8 \AA$ and $1.8 \AA$ away from a carbon atom. The final 200 data points of the training data set also used a flake at $20 \mathrm{~K}$, but the $\mathrm{C}$ atom closest to the projectile was pulled out of the surface by $0.5 \AA$ to $0.8 \AA$ and the $\mathrm{C}$-H distance was set to a value between $1.2 \AA$ and $2 \AA$. The purpose was again extension of configuration space to extreme nonequilibrium geometries. The validation data set consisted of 10,800 reference data points that were calculated during preparation of the training data, but which were not included in it due to the imposed constraints.

The fit itself was performed in following way: the REBO PES was divided into three parts on the basis of the constituent parameter sets. One part describes the $\mathrm{C}$-C interaction, another the projectile-graphene interaction and the last the interaction between the graphene flake and its terminal $\mathrm{H}$ atoms. Note that letting all $\mathrm{H}$ atoms in the simulation cell share a set of parameters would have diminished the role of the projectile due to the sheer numbers advantage of the terminal $\mathrm{H}$ atoms. In the MD simulations which use periodic boundary conditions, there are no terminal $\mathrm{H}$ atoms, so that these parameters can be dropped after a successful fit. For the fit itself and the reproduction of $a b$ initio energies they are of course mandatory. I also used the trust-region nonlinear least squares algorithm which usually reached convergence at around 12 local optimization steps. Literature values were used as the initial parameters to which a Gaussian blur of $15 \%$ was applied. In total there 


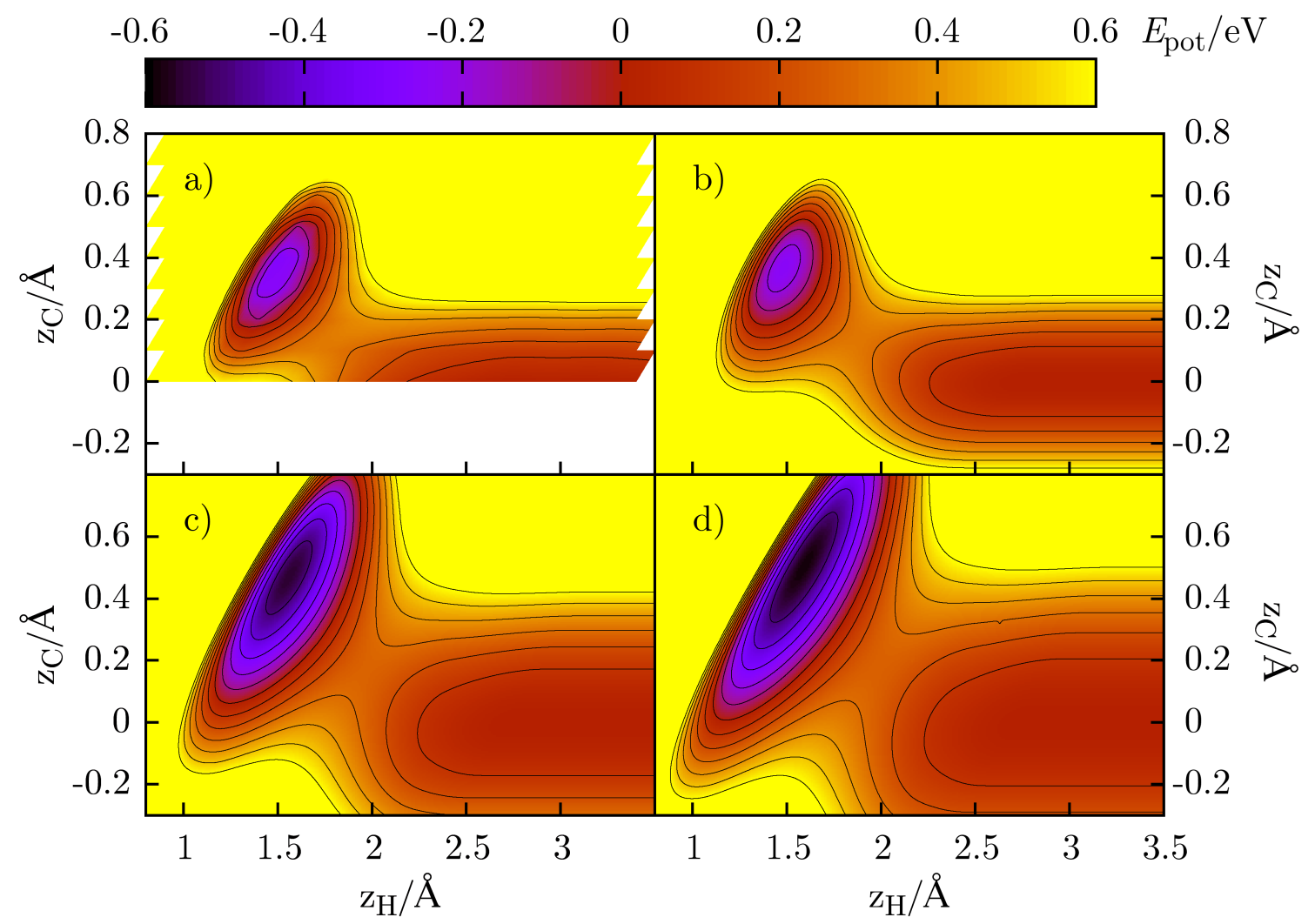

Figure 45: PES of $\mathrm{H}$ on graphene. The $x$-axis shows the distance from the $\mathrm{H}$ atom to the surface plane and the $y$-axis is the $\mathrm{C}$ atom puckering coordinate parallel to the surface normal. Lines of constant energy were added every $100 \mathrm{meV}$ to guide the eye. Panel a) displays ab initio EMFT energies used for visual validation of the parametrized REBO PES shown in panel $b$ ). These two plots are based on grid calculations in which only the $z_{\mathrm{H}}$ and $z_{\mathrm{C}}$ coordinate were scanned. All other DOFs remained fixed. This grid approach is also taken as the basis for panels c) and d), but here the nearest neighbor and next-nearest neighbor carbon atoms, respectively, were allowed to relax using the FIRE algorithm. Each grid point is a square of side length $0.1 \AA$ in panel a) and $0.01 \AA$ in panels b) to d). Cubic splines were used for a smooth color gradient. Barrier heights, chemisorption well depths and their positions are reported in Tab. 9

were 27 parameters which were subject to optimization.

This fitting procedure was computationally much more demanding because on the one hand, there was an additional set of parameters for the description of terminal $\mathrm{H}$ atoms. On the other hand, the number of atoms had more than doubled in comparison to the REBO-PBE PES parametrization. One fit took up to one day depending on the number of Jacobi matrix evaluations and the size of the trust region while simultaneously the training data set size had been reduced. This is also the reason why the compilation of the training set was revised several times in this process and ended up being so complex. There was simply no room for large amounts of geometries covering configuration space in great detail. In consequence, 
Table 9: Barrier heights, chemisorption well depths and their positions extracted from the PES shown in Fig. 45. The more DOFs are relaxed, the lower the barrier to adsorption becomes. Likewise, the chemisorption well becomes deeper. This effect is especially pronounced when nearest neighbor relaxation is switched on: the $\mathrm{C}-\mathrm{H}$ bond more than doubles in strength. The next-nearest neighbors, however, do not play a significant role in terms of equilibrium potential energies.

\begin{tabular}{lcc}
\hline \multicolumn{1}{c}{ Panel in Fig. 45 } & Barrier & Chemisorption well \\
\hline a): EMFT 2D-scan & $330 \mathrm{meV}$ & $294 \mathrm{meV}$ \\
& $z_{\mathrm{C}}=0.16 \AA$ & $z_{\mathrm{C}}=0.36 \AA$ \\
& $z_{\mathrm{H}}=1.74 \AA$ & $z_{\mathrm{H}}=1.51 \AA$ \\
& $326 \mathrm{meV}$ & $252 \mathrm{meV}$ \\
b): REBO-EMFT 2D-scan & $z_{\mathrm{C}}=0.14 \AA$ & $z_{\mathrm{C}}=0.37 \AA$ \\
& $z_{\mathrm{H}}=1.88 \AA$ & $z_{\mathrm{H}}=1.48 \AA$ \\
c): REBO-EMFT 2D-scan & $272 \mathrm{meV}$ & $559 \mathrm{meV}$ \\
1 $^{\text {st }}$ shell relaxation & $z_{\mathrm{C}}=0.17 \AA$ & $z_{\mathrm{C}}=0.47 \AA$ \\
& $z_{\mathrm{H}}=1.97 \AA$ & $z_{\mathrm{H}}=1.56 \AA$ \\
& $266 \mathrm{meV}$ & $591 \mathrm{meV}$ \\
d): REBO-EMFT 2D-scan & $z_{\mathrm{C}}=0.18 \AA$ & $z_{\mathrm{C}}=0.50 \AA$ \\
1 $^{\text {st }}$ and 2 & & \\
& $z_{\mathrm{H}}=1.98 \AA$ & $z_{\mathrm{H}}=1.59 \AA$ \\
\hline
\end{tabular}

early attempts to fit the PES failed. But the described composition of training data points eventually gave reasonable results. The reason for the success is most probably a balance between diversity and sparsity of data points in configuration space which was achieved by observing what was improving the fits, and try and error. The final PES that all collaborators agreed on has an RMSE of $169 \mathrm{meV}$ and is shown in Fig. 45. Its parameters are reported in Tab. 10.

There are only small differences visible between panels a) and b) of Fig. 45. In these two dimensions the PES is accurately fitted. The adsorption barrier and chemisorption well also agree with the reference data as shown in Tab. 9. Performing relaxation calculations was not possible using the EMFT level of theory at the time of this writing. Hence, no direct comparison could be made. It was possible, however, to relax the system using a full B3LYP description and to subsequently obtain the energy of this configuration with EMFT. It can be assumed that the geometries would be very similar since EMFT makes use of B3LYP in the high-level region 
anyway. In this case the chemisorption well depth was calculated to be $590 \mathrm{meV}$ with a barrier to adsorption of $280 \mathrm{meV}$. These values also agree very well with the REBO-EMFT first and second shell relaxation results of Tab.9. It can thus be assumed, that the REBO-EMFT parametrization is a reasonable approach to emulate $a b$ initio EMFT across multiple dimensions of the PES.

The importance of coupled DOFs in this system cannot be stressed enough. The chemisorption well becomes more than twice as attractive as soon as nearest neighbor relaxation is allowed. Also note that the barrier top moves away from the surface plane the more DOFs are relaxed while simultaneously becoming flatter. The nextnearest neighbors still affect the adsorption energies, but much less than the direct neighbors. There are limitations to the use of this PES, however. Since it has only been trained with configurations which are low in energy it should not be used with projectiles of much more than $2 \mathrm{eV}$ incidence energy and the surface should be at a maximum temperature of $500 \mathrm{~K}$. It is still an analytical potential, limited in its flexibility. High accuracy could be achieved in certain PES regions which are important for low energy $\mathrm{H}$ atom scattering simulations. But I want to make clear that this is neither a general purpose carbon potential, nor is it designed to describe hydrocarbons. The PES should only be used for $\mathrm{H}$ atom scattering off of graphene and can be used for any geometries that might be visited during a trajectory simulation.

\subsection{Detailed Dynamics}

Real-time dynamics is of course very different from static cuts through the PES in two dimensions. In this subsection I explain how $\mathrm{H}$ atoms interact with a graphene surface and how they can lose a large portion of their initial kinetic incidence energy upon collision. This system is very different from $\mathrm{H}$ on metal surfaces. The projectile and the surface atoms have a fairly similar atomic mass and directional forces including (re-)hybridization are at play. Though the collisions are described using an electronically adiabatic approach, it will be shown that $\mathrm{H}$ atom energy losses are much higher than predicted by the binary collision model.

\subsubsection{Low Energy Collision}

Before examining scattering events it makes sense to first understand why and how $\mathrm{H}$ atoms can adsorb on free-standing graphene. As soon as this process is clear it can be predicted what needs to be changed in the incidence conditions to avoid or 
Table 10: Optimized REBO-PBE and REBO-EMFT parameter sets. The naming convention is adopted from Ref. [220]. Values are reported with six significant digits, else, the values are exact.

\begin{tabular}{lrrrr}
\hline & \multicolumn{2}{c}{ REBO-PBE } & \multicolumn{2}{c}{ REBO-EMFT } \\
parameter & $\mathrm{C}-\mathrm{H}$ & $\mathrm{C}-\mathrm{C}$ & $\mathrm{C}-\mathrm{H}$ & $\mathrm{C}-\mathrm{C}$ \\
\hline$r_{\min } / \AA$ & 0.0 & 1.75459 & -0.928204 & 1.72471 \\
$r_{\max } / \AA$ & 2.23314 & 1.96393 & 2.75268 & 2.14664 \\
$r_{\max }^{\prime} / \AA$ & 3.20336 & 1.78785 & 2.37499 & 1.92013 \\
$B^{(1)} / \mathrm{eV}$ & 15.1840 & 9840.53 & 41.2768 & 10312.2 \\
$B^{(2)} / \mathrm{eV}$ & 0.0 & 19.5817 & 0.0 & 19.8864 \\
$B^{(3)} / \mathrm{eV}$ & 0.0 & 20.1256 & 0.0 & 24.6564 \\
$\beta^{(1)} / \AA^{-1}$ & 0.681924 & 5.02842 & 1.28144 & 4.85172 \\
$\beta^{(2)} / \AA^{-1}$ & 0.0 & 0.352057 & 0.0 & 1.96277 \\
$\beta^{(3)} / \AA^{-1}$ & 0.0 & 4.37868 & 0.0 & 0.581445 \\
$Q / \AA$ & 0.263106 & 0.063910 & 0.593657 & 0.002 \\
$A / \mathrm{eV}$ & 225.071 & 14154.2 & 165.746 & 8677.31 \\
$\alpha / \AA$ & 4.65647 & 5.31536 & 4.00119 & 4.62961 \\
\hline
\end{tabular}

reduce it. In the end all atoms that stick to the surface reduce the measured signal. To ensure that the projectile adsorbs to the surface I place the $\mathrm{H}$ atom $6 \AA$ above a carbon atom, provide it with an incidence energy of $0.3 \mathrm{eV}$, and launch it directly towards the surface which has a temperature of $0 \mathrm{~K}$. This AIMD trajectory is based on DFT with the PBE functional. The surface consists of only eight carbon atoms of which two are fixed in all dimensions to keep the surface in place. Both of them are at equal distance from the center of impact. The other ones are allowed to move parallel to the surface normal. Since the graphene surface has no thermal energy and the projectile just barely overcomes the barrier changes in the energy can be attributed to the adsorption process. After the AIMD simulation finishes I perform singlepoint energy calculation of the configurations visited during the trajectory with the $\mathrm{H}$ atom removed. This yields the deformation energy of graphene $E_{\text {def }}(\mathrm{C}-\mathrm{C})$. With the total potential energy $E_{\text {pot }}$ during the trajectory and the deformation energy at hand the $\mathrm{C}-\mathrm{H}$ interaction energy can be calculated by

$$
E_{\text {int }}(\mathrm{C}-\mathrm{H})=E_{\text {pot }}-E_{\text {def }}(\mathrm{C}-\mathrm{C}) .
$$




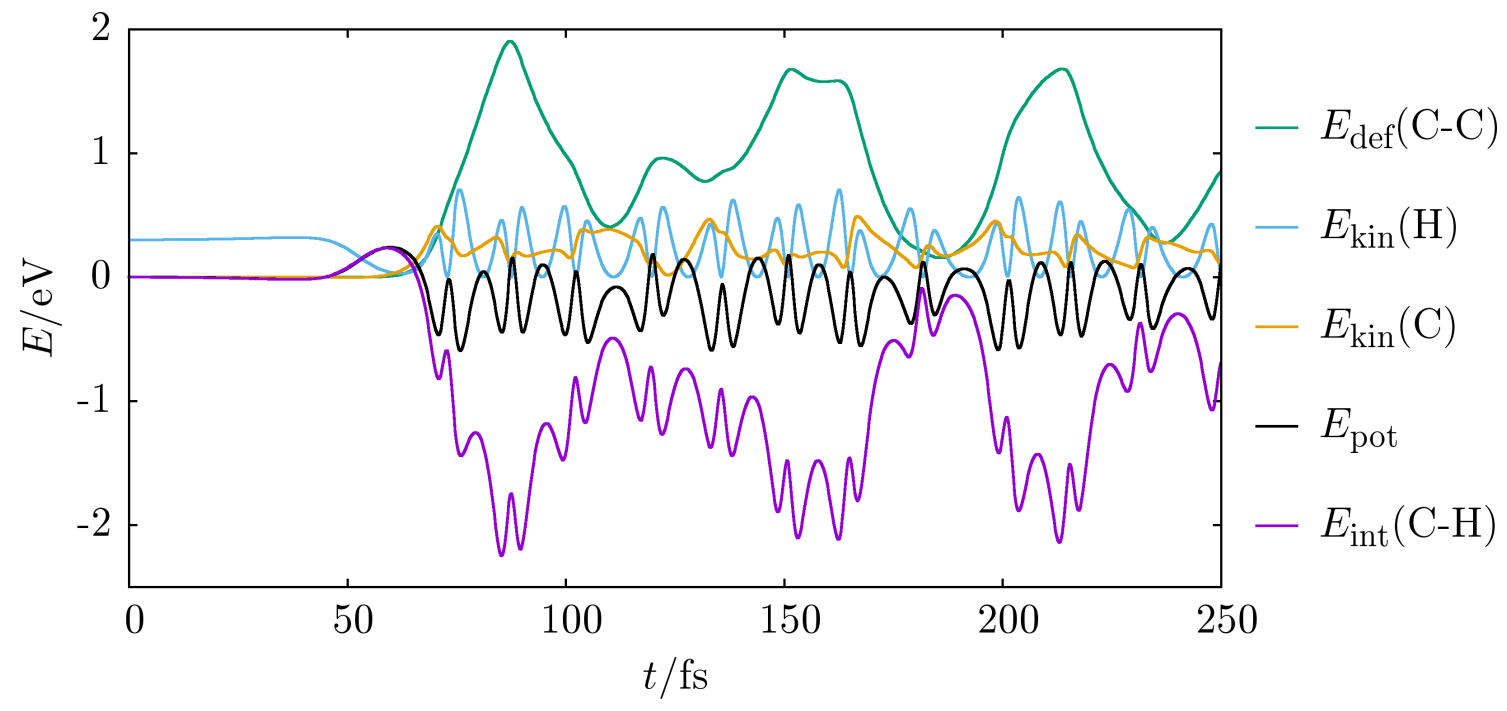

Figure 46: Graphene deformation energy (green curve), projectile (blue curve) and graphene network (orange curve) kinetic energy, H-graphene interaction energy (purple curve) during an AIMD trajectory with $E_{\mathrm{i}}=0.3 \mathrm{eV}$ and $\theta_{\mathrm{i}}=0^{\circ}$. The gain in total potential energy, $E_{\text {pot }}=E_{\text {def }}(\mathrm{C}-\mathrm{C})+E_{\text {int }}(\mathrm{C}-\mathrm{H})$, due to adsorption seldom exceed $0.5 \mathrm{eV}$ (black curve). This is due to compensation between $\mathrm{C}-\mathrm{H}$ interaction energy and surface deformation caused by re-hybridization and subsequent $\mathrm{C}$ atom puckering. Quickly after adsorption, a balance between projectile and surface kinetic energy is established.

This dissection of energy curves is shown in Fig. 46. Any energies of formation are constant and have simply been removed from the plot.

Interestingly enough, $E_{\text {int }}(\mathrm{C}-\mathrm{H})$ and $E_{\text {def }}(\mathrm{C}-\mathrm{C})$ both have a maximum amplitude of approximately $2 \mathrm{eV}$. They also nearly compensate each other so that the $\mathrm{H}$ atom is bound to the surface with 0 to $0.5 \mathrm{eV}$. Typical $\mathrm{C}-\mathrm{H}$ bond dissociation energies in small organic molecules are about eight times that value. Also note how the green and purple curves in Fig. 46 change drastically in the time after the adsorption event. The total potential energy which is the sum of both, however, remains on the order of the graphene kinetic energy. A superposition of the short $\mathrm{C}-\mathrm{H}$ and longer $\mathrm{C}-\mathrm{C}$ period of oscillation is intuitively visible from the purple curve. Starting with the green curve directly after adsorption, the $\mathrm{C}$ atom starts to pucker out of the surface, simultaneously increasing both the deformation and H-graphene interaction energy. At points of maximum deformation energy it is furthest out. As an immediate consequence of Fig. 45 one cannot occur without the other. The minimum energy pathway to adsorption couples the $z_{\mathrm{H}}$ and $z_{\mathrm{C}}$ coordinates which leads to a synchronized motion of both collision partners. In the end the PES which the system is moving on is not given by any single energetic contribution. It is rather the total potential energy of the system which turns out to slightly favor the 


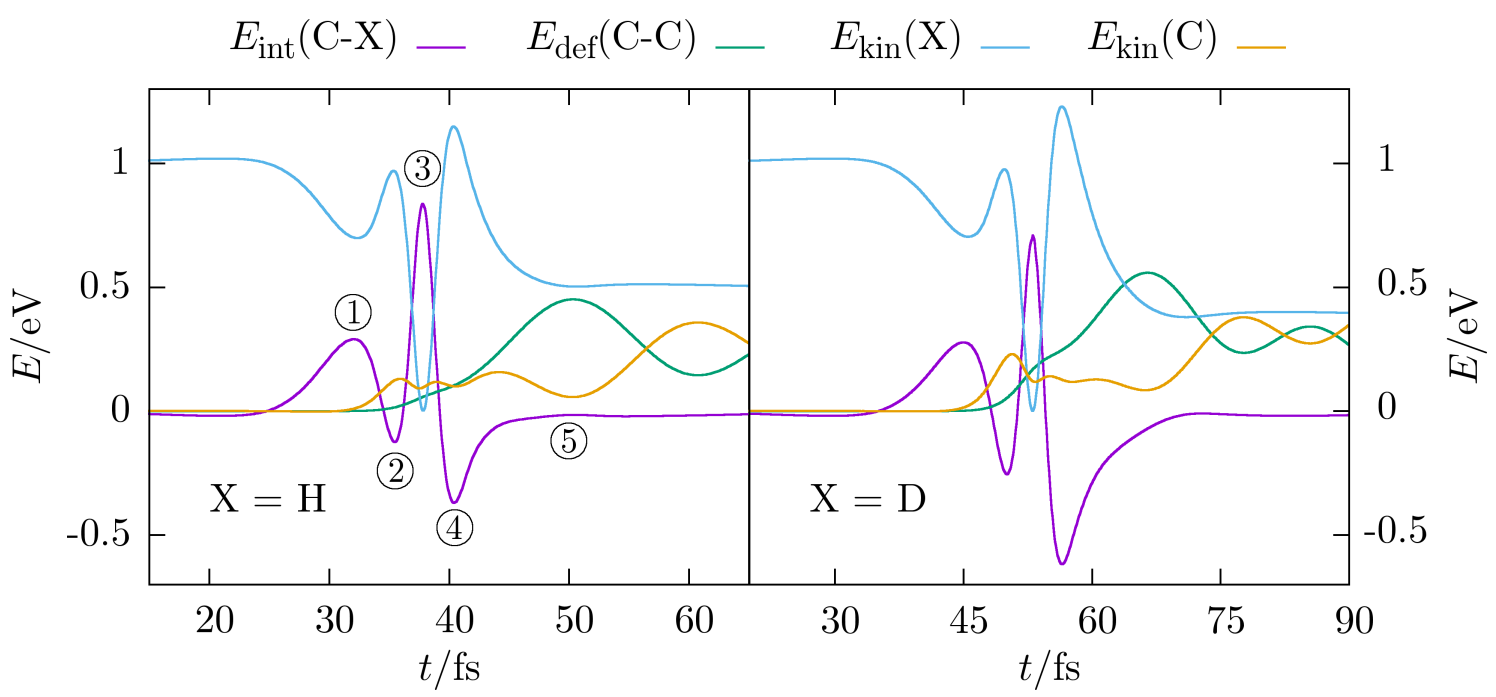

Figure 47: Energetic contributions during an H (left panel) and D (right panel) AIMD trajectory scattering from free-standing graphene. The projectile has a initial kinetic energy of $1 \mathrm{eV}$ and is launched parallel to the surface normal directly on top of a carbon atom. The surface has an initial temperature of $0 \mathrm{~K}$ and consist of eight carbon atoms. Different points in time in the left panel are marked to be referenced from the text. The different contributions are the $\mathrm{C}-\mathrm{H}$ interaction energy (purple curve), the graphene strain energy (green curve), the kinetic energy of the incoming particle (blue curve) and the surface atoms (orange curve) which were obtained from central differences of the atomic coordinates. The beginning and the surface equilibration phase at the end of the trajectories are left out to focus entirely on the projectile-surface interaction. All lines add up to $1 \mathrm{eV}$ at each time step since this is the amount that the incident particle brought with it.

puckered and adsorbed state by the mentioned 0 to $0.5 \mathrm{eV}$.

\subsubsection{Medium Energy Collision}

The medium energy range around $1 \mathrm{eV}$ normal incidence energy is most interesting in terms of energy transfer. At lower energies everything sticks if the $\mathrm{C}$ atom is directly hit and at higher energies the collision happens so quickly that the graphene network has no time to react. At a medium energy range there is also the most pronounced isotope effect which will be discussed in this paragraph. A figure similar to the presented energy curves of low energy $\mathrm{H}$ atom adsorption is shown in Fig. 47. It contains two panels with four curves each. The left one describes an $\mathrm{H}$ atom provided with $E_{\mathrm{i}}=1 \mathrm{eV}$ and $\theta_{\mathrm{i}}=0^{\circ}$ scattering from the top-site of a free-standing graphene surface.

After being created the $\mathrm{H}$ atom experiences the following series of events: in the first $25 \mathrm{fs}$ it approaches the surface and reaches the top of the barrier shortly after $30 \mathrm{fs}$. This point corresponds to (1) in the left panel of Fig. 47. At this point the energy for climbing the barrier has been taken entirely from the kinetic energy of the 
$\mathrm{H}$ atom. At (2) the projectile reaches the very shallow chemisorption well. It regains nearly all of its initial kinetic energy simply by continuing its motion downhill from the barrier top further towards the surface. This is also the point at which the target $\mathrm{C}$ atom starts to move towards the incoming particle thereby introducing the first signs of strain to the graphene network. (3) indicates the closest H-surface approach. Here the $\mathrm{H}$ atom is at the highest point of the $\mathrm{C}-\mathrm{H}$ bond repulsive wall. The kinetic energy of its collision partner slightly dips at this particular time because it experiences a head-on collision. Simultaneously, the surface deformation energy assumes a value different from zero for the first time caused by the $\mathrm{C}$ atom motion mentioned in the previous point.

Next, the projectile is repelled from the surface and this time finds at deeper chemisorption well at (4). By now the surface has been in contact with the incoming particle for about $10 \mathrm{fs}$. It had some time to adapt its geometry and due to the slightly puckered $\mathrm{C}$ atom the $\mathrm{C}-\mathrm{H}$ interaction is more attractive. Note that at this point, the projectile has more kinetic energy than it was initially created with. The surface kinetic energy feeds on C-H interaction energy and leads to graphene network deformation. The biggest contributors in this restrained simulation are the target $\mathrm{C}$ atom moving up and its three neighbor atoms moving down. All of them also store deformation energy by approximating $\mathrm{sp}^{3}$ hybridization of the central $\mathrm{C}$ atom. At (5), the deformation energy reaches its maximum, driven by the $\mathrm{H}$ atom having broken the $\mathrm{C}-\mathrm{H}$ bond and the inertial carbon atom motion. In the end, the projectile has caused the slab to absorb half its initial kinetic energy. $\mathrm{H}$ atom kinetic energy is converted alternatingly to attractive and repulsive interaction energy. The created transient bond, however, dissipates part of that energy into surface kinetic energy which in turn causes deformation. The two forms of energy are quickly delocalized over the graphene network so that the $\mathrm{H}$ atom has no chance of gaining it back when being reflected.

Another interesting phenomenon becomes evident when comparing $\mathrm{H}$ to $\mathrm{D}$ atom scattering in Fig. 47. Having the same incidence energy D travels by a factor of $\sqrt{2}$ slower than H. In consequence the surface has more time to adapt to the approaching particle. This becomes especially evident when comparing the minima of the projectile-surface interaction energy. The purple curves in both panels have two minima, but they are deeper both times in the right panel showing D atom scattering. In turn the heavier isotope has even more energy for a few femtoseconds than it initially started with. This would correspond to (4) in the $\mathrm{H}$ atom panel and marks the position at the bottom of the chemisorption well after being reflected from the 


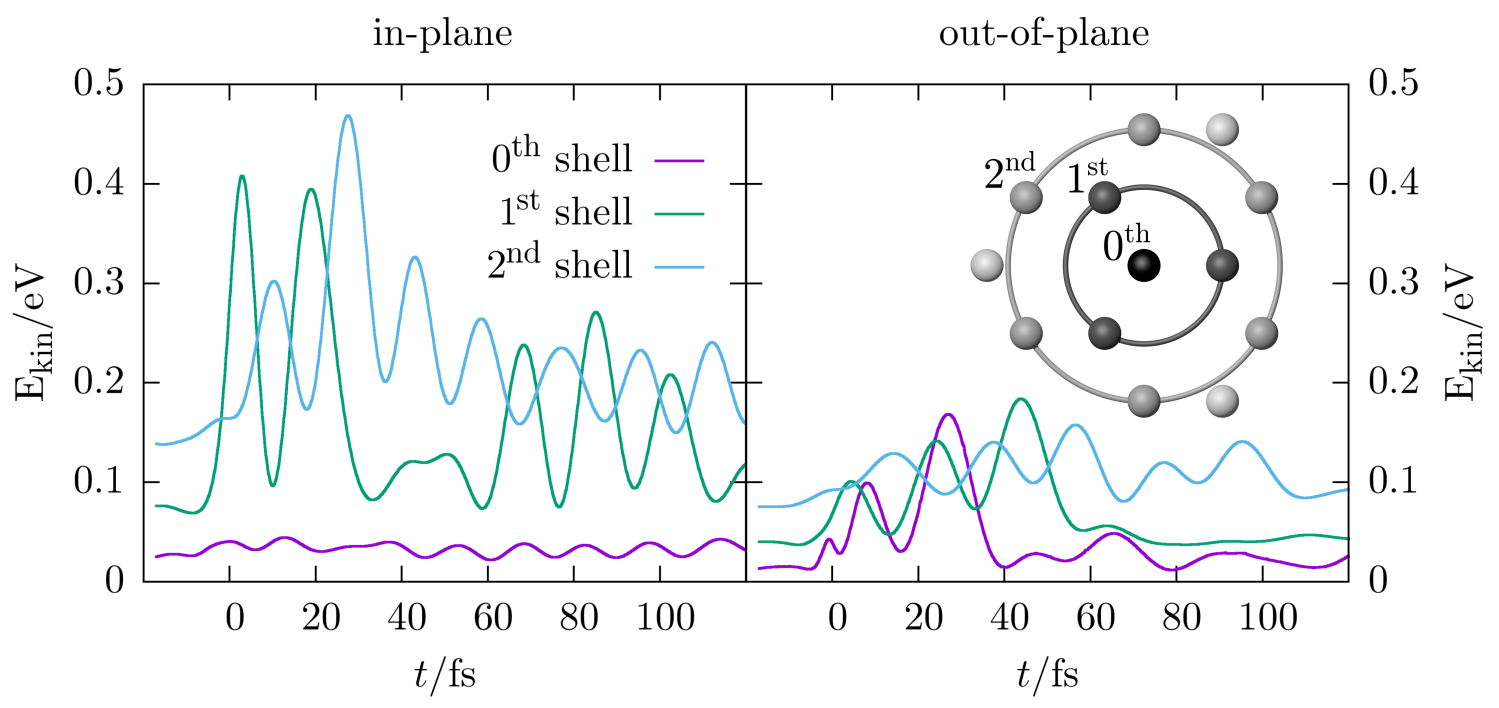

Figure 48: Average kinetic energy propagation in the graphene surface based on 60 trajectories in which an on-top collision occurs. A trajectory is a labeled as such if at the moment of closest $\mathrm{C}-\mathrm{H}$ approach, one $\mathrm{C}-\mathrm{H}$ distance is less than $1.15 \AA$ and exactly three other $\mathrm{C}-\mathrm{H}$ distances are between 1.6 and 2.0 $\AA$. The incident $\mathrm{H}$ atom also has to scatter after a single bounce. These curves are obtained with the REBO-EMFT PES from trajectories in which $E_{\mathrm{i}}=1.92 \mathrm{eV}, \theta_{\mathrm{i}}=35^{\circ}$, $\varphi_{\mathrm{i}}=\mathcal{N}\left(13.5^{\circ} ; 2.12^{\circ}\right)$ and the surface is at a temperature of $300 \mathrm{~K}$. Before averaging, the kinetic energy curves were shifted so that the point of closest approach is at $t=0 \mathrm{fs}$. Each curve represents the spread of kinetic energy in the surface in terms of atomic shells surrounding the target $\mathrm{C}$ atom called $0^{\text {th }}$ shell. In the left panel, the energy absorbed by the $\mathrm{C}$ atom motion in the $x y$-plane is depicted while the right panel shows the out-of-plane portion. Both are shown with the same $y$-axis scale to facilitate comparisons. The inset shows the $\mathrm{C}$ atoms around the collision center and the mentioned shell structure.

surface. Since the collision event as a whole lasts longer there is more time to convert $\mathrm{H}$ atom incidence and $\mathrm{C}-\mathrm{H}$ bond energy into delocalized graphene deformation energy. This can be seen in the higher projectile energy loss. At the end of both trajectories the $\mathrm{H}$ atom lost $0.5 \mathrm{eV}$ whereas its heavier isotope lost $0.6 \mathrm{eV}$.

In a more realistic simulation scenario with the surface at a finite temperature and a non-zero polar angle the collision does not happen collinearly. This complex system gives rise to an energy loss distribution and also the sticking probability is more difficult to predict. Nevertheless, the basic mechanisms discussed here still apply. As the $\mathrm{H}$ atom approaches one of the $\mathrm{C}$ atoms the $\mathrm{C}$ atom comes out of the plane of the graphene to form a chemical bond to the $\mathrm{H}$ atom. The next-nearest neighbor $\mathrm{C}$ atoms begin moving in the plane away from the $\mathrm{C}$ atom being attacked. This concerted motion of four $\mathrm{C}$ atoms accounts for the very large energy losses which has extensively been discussed in Ref. [6]. Fig. 48 illustrates the importance of in-plane carbon atom motion which also appears in this reference. One can see that the collision with the surface triggers a very quick response of the graphene 
network. In the left panel the first and second shell in-plane motion is excited about $10 \mathrm{fs}$ after impact. This is because the re-hybridization does not only cause the central $\mathrm{C}$ atom to pucker, but it also elongates the bonds to its neighbors. In line with the inverse relationship between bond length and bond order [236] the target $\mathrm{C}$ atom pushes its neighbors away as the re-hybridization progresses. This is also felt by the next-nearest neighbors which in turn accept and further transport the energy away from the collision site. The target $\mathrm{C}$ atom itself experiences no significant influence on its kinetic energy in the graphene plane because the projectile hits ontop. It does, however, start to move in the out-of-plane direction which can be seen in the right panel of Fig. 48. The energy it accepts is even comparable to that of its neighbors, but it is less than half of the distributed in-plane kinetic energy and there are two reasons for it. First the in-plane motion represents a longitudinal phonon that has a shorter wavelength at maximum excitation than the transversal out-ofplane phonon. Second there are two dimensions available for the in-plane motion, but just one for the out-of-plane motion. Hence, there are more DOFs available in-plane which naturally can take up more energy.

\subsubsection{High Energy Collision}

The last paragraph of the H-graphene interaction trilogy concerns scattering at a high incidence energy. High in this context means about $2 \mathrm{eV}$ which corresponds to the fastest experimentally generated projectiles. The familiar interaction plot is shown in Fig. 49. Surprisingly enough only a very small projectile energy loss can be observed. Comparing with the previously discussed incidence conditions, contrary to intuition, it seems the lower the particle's initial energy, the more it loses upon contact with the graphene surface. A closer look at the various curves given some important insights about why so little energy is lost in this case.

Naively, one would argue that the velocity of the projectile is responsible for a reduced interaction time. Combined with the carbon atoms' inertia only a small distortion of the graphene network can occur. Hence, if the energy is not taken up by the surface it has to stay with the projectile. A sound reasoning, but this is not what happens. One finds that the interaction time, which can be approximated by the duration of a non-zero $E_{\text {int }}(\mathrm{C}-\mathrm{H})$ value, is nearly independent of whether the projectile is provided with 1 or $2 \mathrm{eV}$. What in fact leads to an increased energy transfer by prolonging the interaction time is the mass. This became evident in the paragraphs about medium energy scattering. The observations can be explained 


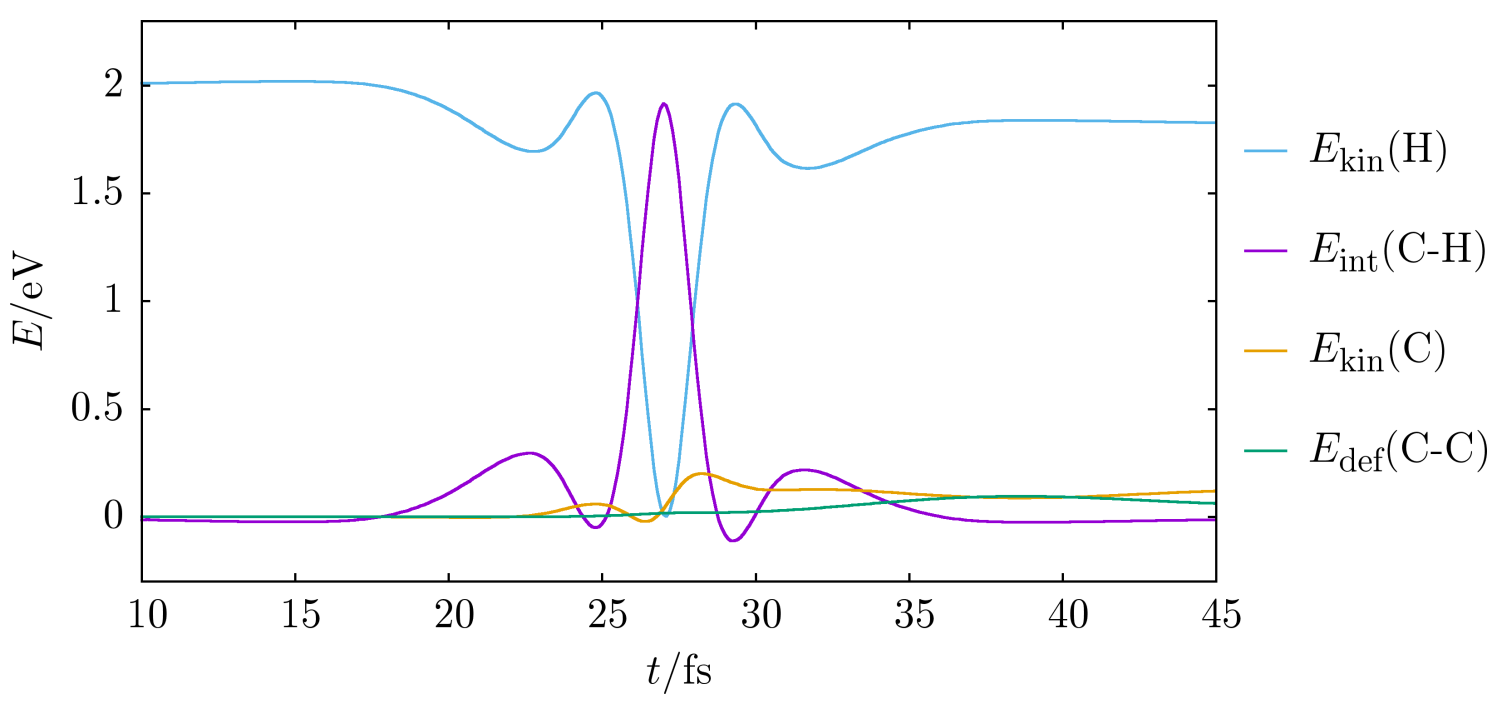

Figure 49: Graphene deformation energy (green curve), projectile (blue curve) and graphene network (orange curve) kinetic energy, H-graphene interaction energy (purple curve) during an AIMD trajectory with $E_{\mathrm{i}}=2 \mathrm{eV}$ and $\theta_{\mathrm{i}}=0^{\circ}$. Only a small amount of energy can be taken up by the graphene surface. This is a surprising finding, given the fact that projectile-surface interaction time is very similar to the $1 \mathrm{eV}$ scattering simulation displayed in the left panel of Fig. 47.

by drawing an analogy between our system of interest and a classical harmonic oscillator.

It is well known that the period of oscillation $T$ in a harmonic oscillator is independent of its initial displacement. In the formula for $T$ there only exists a factor of $\sqrt{m / k}$ with $m$ as the mass and $k$ as the spring constant. Since H and D are moving on the same PES $k$ is the same for both particles. However, $m$ changes by a factor of two which directly translates to an increased interaction time from approximately 20 fs for $\mathrm{H}$ in Fig. 47 to $\sqrt{2} \cdot 20 \mathrm{fs} \approx 28 \mathrm{fs}$ for D atom scattering. For D it is actually even a few femtoseconds more since is ends up with less kinetic energy than $\mathrm{H}$ whereas the mentioned relationship between $T$ and $m$ is only valid for an undamped system. This behavior qualifies $\mathrm{H}$ and $\mathrm{D}$ atoms scattering from graphene as a direct positive feedback loop system in which a longer interaction time causes more energy loss and vice versa.

A more careful analysis of the curves in Fig. 49 reveals the following mechanism: at around 25 fs everything looks as expected. The $\mathrm{H}$ atom sacrifices some of its kinetic energy to pass over the barrier and finds an extremely shallow chemisorption well. It then regains its energy before colliding with the repulsive wall. This causes some $\mathrm{C}$ atom motion which, surprisingly, does not lead to much lattice distortion. The clue is just shortly before the $\mathrm{H}$ atom reaches its highest position on the repulsive potential, that is when it has lost all of its energy for one moment, the graphene 
kinetic energy goes to zero. At this point in the trajectory the target $\mathrm{C}$ atom jerks to a halt in its puckering motion. Shortly thereafter an increase in the surface kinetic energy is visible as the $\mathrm{H}$ atom starts moving away from its collision partner. This increase is due to the target $\mathrm{C}$ atom quickly moving downward. The incident particle has simply so much momentum that it reverses the carbon atom's direction of travel.

Without any puckering no transient $\mathrm{C}-\mathrm{H}$ bond can form which is why there is barely any attractive interaction visible between the colliding particles. It also means that energy transfer to the surface is inefficient at such high incident energies. Since re-hybridization and subsequent lattice distortion are missing in this collision event the projectile only loses a small fraction of its energy. It can in fact be estimated from the binary collision model that to cause the experienced energy loss of $200 \mathrm{meV}$ the $\mathrm{H}$ atom would have to collide with another atom of three times the mass of carbon. This a reasonable number since the $\mathrm{C}$ atom is embedded in an extended surface that effectively increases the mass of every single atom.

\subsection{Sticking}

In this subsection sticking of $\mathrm{H}$ atoms on the graphene surface shall be further analyzed and quantified. I will show to what extent quantum effects matter in the adsorption process, how isotope substitution influences the process and what effect an addition of a metal substrate has on the sticking probability. The REBOEMFT potential will be largely used for this purpose since it was parametrized using higher level reference data. Nevertheless, all mentioned trends and observations also apply to the REBO-PBE potential. Both potentials are qualitatively similar usually resulting in a $10 \%$ shift to lower energies using REBO-PBE since the barrier is a bit lower and the $\mathrm{C}$ atom needs slightly less energy to pucker.

\subsubsection{Comparison with EMFT}

Fig. 50 shows a comparison between sticking probabilities calculated with ab initio EMFT and the parametrized REBO-EMFT potential. The REBO-EMFT data is obtained by using an incidence energy of $1.92 \mathrm{eV}$ and varying the polar incidence angle $\theta_{\mathrm{i}}$. This way the normal component of the energy can be changed. EMFT sticking probabilities are calculated in the same way except for the two data points on the lower end of the normal incidence energy axis. Due to the finite size of the flake in these calculations the incoming particle would have to travel over the 


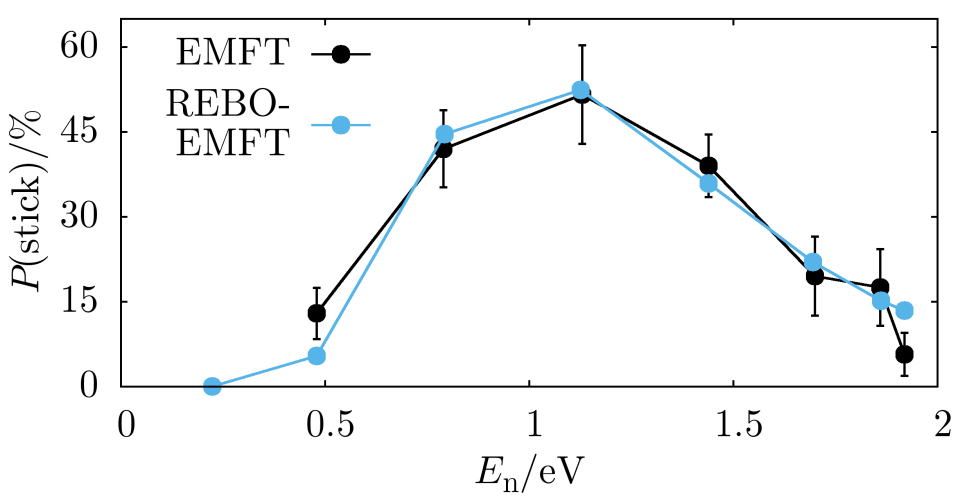

Figure 50: Sticking probability of $\mathrm{H}$ on graphene at $300 \mathrm{~K}$. Incidence conditions are $E_{\mathrm{i}}=$ $1.92 \mathrm{eV}, \varphi_{\mathrm{i}}$ is random and $\theta_{\mathrm{i}}$ was varied to arrive at different normal incidence energies. Blue data points are calculated with the parametrized REBO-EMFT potential and the black ones correspond to the $a b$ initio EMFT reference method. Error bars indicate the $95 \%$ confidence interval.

embedding boundary at high incidence angles which destabilizes the EMFT wave function. Therefore, the two data points in question were obtained with a fixed $\theta_{\mathrm{i}}=40^{\circ}$ and the total incidence energy was reduced so that its normal component matches the desired values of 0.48 and $0.79 \mathrm{eV}$.

The EMFT data, given the fact that these sticking probabilities are based on over 1,200 AIMD trajectories, only became available after the REBO-EMFT parametrization procedure had finished. It is not part of the original validation data set, but serves as a test set in machine learning terminology. This means that the model neither learns from it nor does it influence the model's hyperparameters. This EMFT sticking probability curve allows for a final and unbiased evaluation of the REBOEMFT PES performance. The focus here is especially on the dynamic aspects of the parametrization as both energies and forces must be reproduced to end up with similar sticking probabilities. The agreement between both curves in Fig. 50 is extremely good. It is much better than one would expect given the RMSEs to training and validation data and taking into account the complexity of the PES. Error cancellation cannot be entirely excluded, however, it is unlikely given the $1.5 \mathrm{eV}$ energy range over which the data was collected. In any case it provides a good overview about the curve shape of the sticking probability and also absolute numbers which will serve as a reference when examining other influences on this quantity.

\subsubsection{Nuclear Quantum Effects}

Next, the influence of Nuclear Quantum Effects (NQEs) shall be characterized. For this purpose Ring Polymer Molecular Dynamics (RPMD) is used. The simulations run on the same PES as classical dynamics, but they sample an extended phase space. Background information about this approach including the equations of mo- 


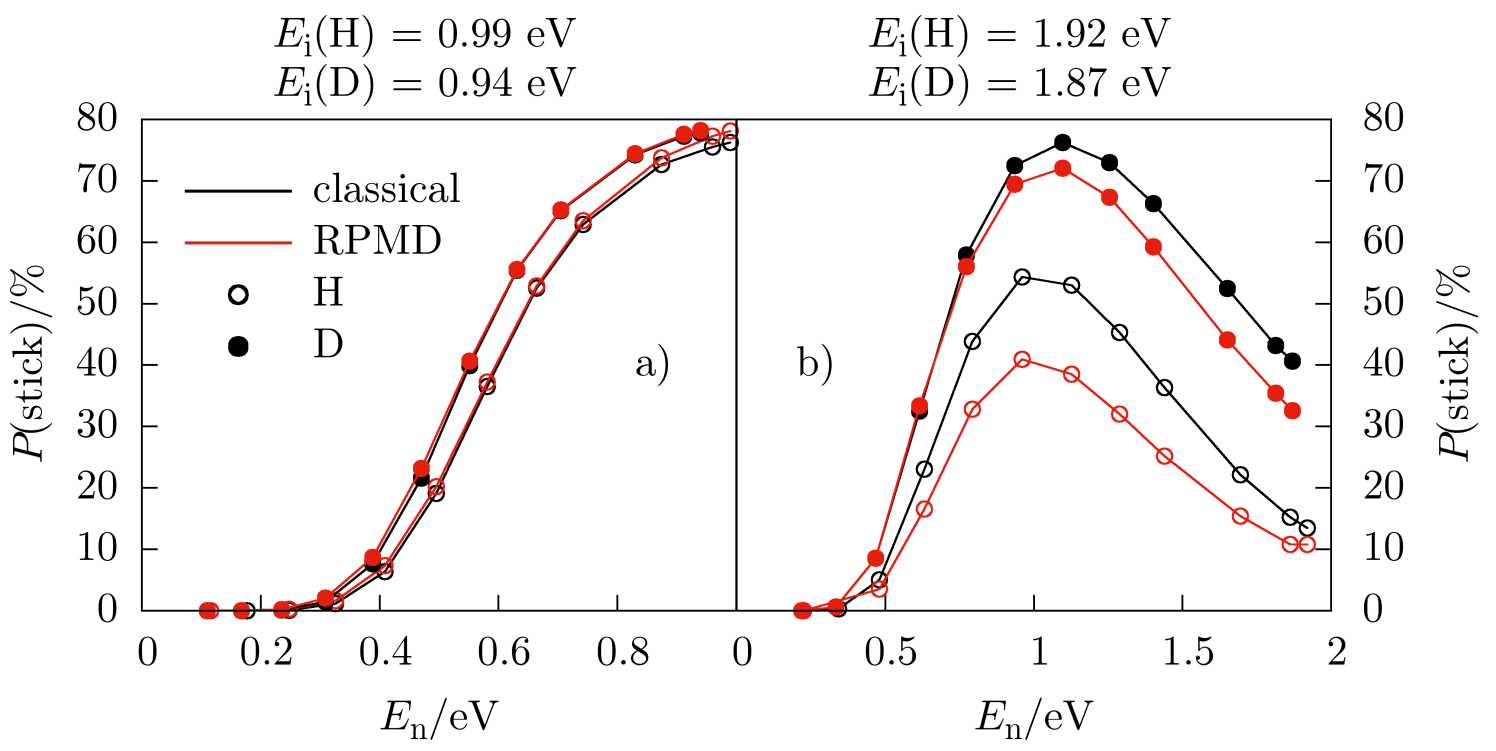

Figure 51: Sticking probabilities of $\mathrm{H}$ and D on graphene. Open circles indicate $\mathrm{H}$ whereas filled circles symbolize its heavier isotope. The black curve shows classical results and the red curve displays results obtained with RPMD. The incidence energies of the projectiles are provided above the panels and different normal energies are realized by changing $\theta_{\mathrm{i}}$. $\varphi_{\mathrm{i}}$ is given by $\mathcal{N}\left(13.5^{\circ} ; 2.12^{\circ}\right)$ with respect to a $\mathrm{C}-\mathrm{C}$ bond and the surface is at $300 \mathrm{~K}$ in all cases.

tion can be found in section 2.4.3. It is a parameter-free theory that can be derived from Matsubara dynamics which, in turn, can be derived from first principles. In the simulations the Ring Polymer (RP) centroid mode which describes the movement of the ring polymer as a whole is given the incidence energy of a classical particle. This is the only reasonable choice [237] since quantum kinetic energy is not present in the projectile at the start of a trajectory because it is not interacting with the surface yet. However, the impinging, hyper-thermal projectile requires the introduction of a temperature for the internal modes. The optimum value to use can be derived from the energy spread present in each $\mathrm{H}$ atom pulse in experiment. This spread is due to the size of the dissociation laser beam's spacial and spectral coherence [238, 239]. From a relative velocity point of view the average atom in a pulse travels exactly at the desired incidence energy. Therefore, this amount gets deposited in the centroid mode. The pulse shape can be approximated by a Gaussian, and from its standard deviation, a temperature of $300 \mathrm{~K}$ can be derived for the internal bead temperature.

Fig. 51 shows how the sticking probability depends on the isotope and whether or not NQEs are taken into account. The incidence energy in each panel is fixed and listed above it. $\theta_{\mathrm{i}}$ was again varied to achieve different normal energies and $\varphi_{\mathrm{i}}$ was normally distributed with $\mu=13.5^{\circ}$ and $\sigma=2.12^{\circ}$ with the direction of a $\mathrm{C}-\mathrm{C}$ bond for reasons that I will make clear soon. In the classical simulations 
the graphene surface was brought to $300 \mathrm{~K}$ using the Andersen thermostat and the RPMD initial configurations were obtained with the Path Integral Langevin Equation (PILE) thermostat.

Surprisingly in panel a) of Fig. 51 which shows the sticking probability at the lower incidence energy there are only tiny differences between classical and RPMD results. The $\mathrm{H}$ and $\mathrm{D}$ atom sticking curves look very similar and are shifted horizontally by approximately $50 \mathrm{meV}$. In all cases sticking can only occur when a $\mathrm{C}$ atom is hit. All projectiles that aim at the center of the six-membered carbon ring are scattered. The slightly higher sticking probability of D can be attributed to a marginally larger catchment area. It is just a bit larger because of a smaller barrier and better energy transfer to the carbon lattice. This basis for it is, again, the smaller velocity of the heavier isotope just as described in the paragraph about medium energy adsorption in AIMD.

In general it is not straightforward to say what influence isotope substitution has on the energy loss. In principle the barrier should be higher for $\mathrm{H}$ in any case. First because it moves faster than $\mathrm{D}$ which gives the graphene less time to adapt and second it has a higher Zero-Point Energy (ZPE) at the transition state due to confinement effects. On the other hand it has a higher probability of tunneling though the barrier. In principle there is also quantum mechanical over-barrier reflection, but RPMD does not capture this effect. Concerning the chemisorption well region it has been shown theoretically [240, 241] and in experiment [242] that the C-H bond has a ZPE of $305 \mathrm{meV}$ whereas it is only $216 \mathrm{meV}$ for the C-D bond. The depth of the chemisorption well, however, depends very much on incidence energy. As shown in Fig. 47 the depth can vary within a few femtoseconds since it is largely determined by the $\mathrm{C}$ atom puckering motion.

With this line of argumentation D should experience a more powerful collision for it has the smaller ZPE and sees a deeper well due to its smaller velocity. Arguably though it might be only a minor effect because energy is largely lost via re-hybridization followed by lattice distortion. This is very different from a classical binary collision where the amount of energy is directly proportional to the energy at impact. Hence, there is no clear trend caused by isotope substitution concerning sticking and energy loss because there are many competing effects at play simultaneously.

Understanding the reason why there is no visible manifestation of NQEs in the left panel of Fig. 51 is more involved. Especially because this panel shows sticking probabilities at low incidence energies one would intuitively expect them to play a 
larger role than in panel b). However, the results with and without NQEs nearly overlap perfectly for both $\mathrm{H}$ and $\mathrm{D}$ atoms. In principle the projectile can end up in three scenarios under these incidence conditions shown in the said panel. At very low normal incidence energies it is scattered from both the ring center and the barrier to $\mathrm{C}-\mathrm{H}$ bond formation. Therefore, no sticking occurs in this energy region. With higher normal incidence energies the catchment area above the $\mathrm{C}$ atoms first appears and then becomes larger leading to more sticking. An escape after a direct collision is only possible when $E_{\mathrm{n}}>0.8 \mathrm{eV}$. This means over large parts of this panel once a C-H or C-D bond has been formed it cannot be broken.

With this in mind the ZPE in the chemisorption well does not need to be considered and the only two remaining NQEs are tunneling through the barrier and ZPE at the transition state. Both of them should only play a role near the barrier energy in panel a) of Fig. 51. This can in fact be seen from the classical and quantum sticking probabilities $P_{\mathrm{H}, \mathrm{cl}}=1.17 \%, P_{\mathrm{H}, \mathrm{qu} .}=1.66 \%, P_{\mathrm{D}, \mathrm{cl} .}=1.46 \%, P_{\mathrm{D}, \mathrm{qu} .}=2.08 \%$ at $\theta_{\mathrm{i}}=55^{\circ}$ corresponding to $E_{\mathrm{n}}=326 \mathrm{meV}$ for $\mathrm{H}$ and $E_{\mathrm{n}}=309 \mathrm{meV}$ for D. When NQEs are taken into account the sticking probability, a clear sign of barrier crossing, increases for both isotopes which allows for the following conclusions: If there is an (inverse) kinetic isotope effect (KIE) stemming from the quantum nature of the system, it must be very small because the higher sticking probability for D atoms is present with and without NQEs. There is no doubt about the inertial KIE which enhances D atom sticking. Tunneling through the barrier accelerates the reaction more than ZPE at the transition state slows it down. This is the true for both isotopes.

Paris et al. [243] quantified the ZPE at the transition state using the coronene molecule described by B3LYP and the cc-pVDZ basis set. They arrived at a reasonable barrier to adsorption of $294 \mathrm{meV}$ which increases to $337 \mathrm{meV}$ and $315 \mathrm{meV}$ for $\mathrm{H}$ and $\mathrm{D}$ atoms, respectively. This $22 \mathrm{meV}$ difference corresponds to modern "chemical accuracy" divided by two. $22 \mathrm{meV}$ is a very small amount of energy which is far from the accuracy of physically motivated analytical PESs. Nevertheless, it is in an energy range that tunneling $\mathrm{H}$ atoms might well compensate.

The adsorption process at lower energies as seen with the eyes of a ring polymer is shown in Fig. 52 . The $x$-axis shows the RP's center of mass and the $y$-axis shows how its gyration radius parallel to the surface normal changes as it approaches the surface. The radius of gyration in the $z$-direction $r_{z}$ is shown in relation to its value at equilibrium to better compare the two isotopes. Usually, a value below unity means the RP is influenced by attractive interactions with other particles and above 


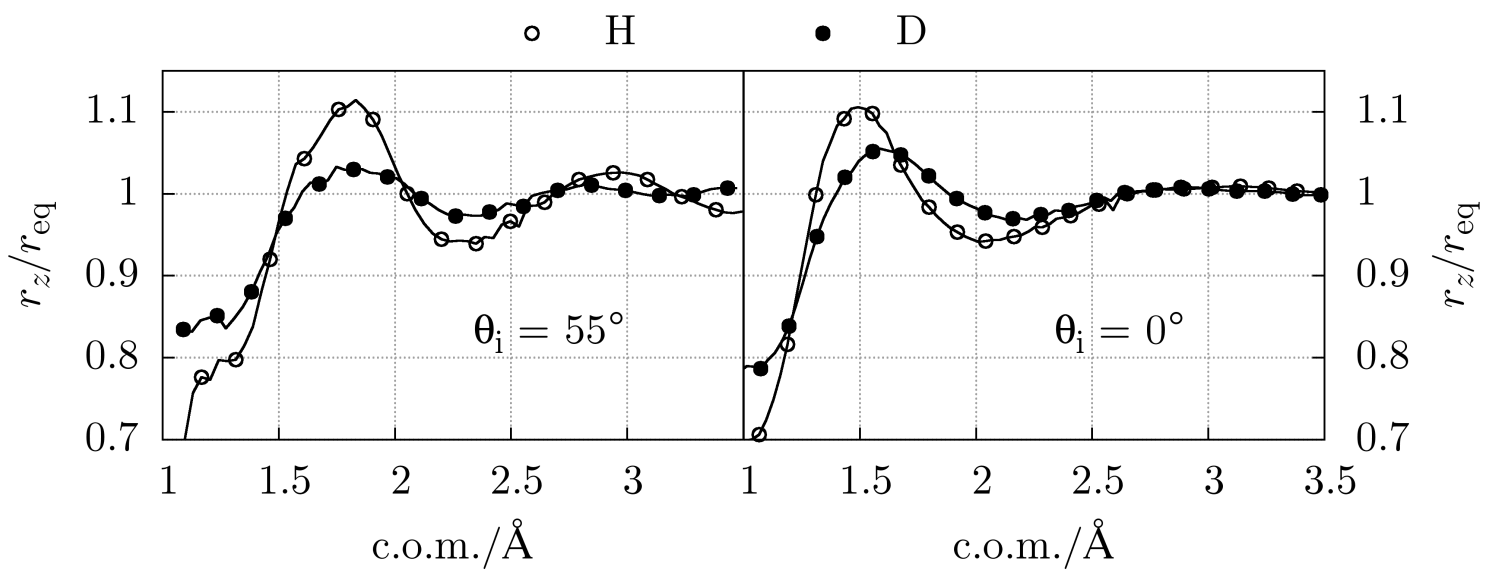

Figure 52: Ratio between the radius of gyration $r_{z}$ in $z$-direction and its equilibrium value $r_{\text {eq }}$ as the RP approaches a free-standing graphene surface at $300 \mathrm{~K}$. The $x$-axis shows the RP's center of mass position. Open circles indicate $\mathrm{H}$ atoms and closed circles represent $\mathrm{D}$ atoms. The polar incidence angle is shown in each panel and the incidence kinetic energy of $\mathrm{H}$ atoms is $E_{\mathrm{i}}=0.99 \mathrm{eV}$ and $E_{\mathrm{i}}=0.94 \mathrm{eV}$ is chosen for $\mathrm{D}$ atoms in both panels. Only those trajectories are taken into consideration which lead to sticking and data collection is stopped as soon as the projectile reaches its first turning point. In the left panel the $\mathrm{H}$ atom curve is based on 3,900 trajectories and the $\mathrm{D}$ atom curve is the average ring polymer behavior in 4,200 trajectories. Calculation of 200,000 trajectories for each isotope were necessary for this panel because the sticking probability is very low at this incidence condition. In the right panel the curves of $\mathrm{H}$ and D are based on 16,500 and 16,900 trajectories, respectively, for which only 20,000 trajectories were required per isotope.

unity indicates a repulsive environment and possibly some beads find themselves in a classically forbidden region of the PES. Unfortunately, Fig. 52 is the only chance there is to directly observe tunneling in a ring polymer.

The incidence conditions in the left panel are chosen such that the sticking probability is around $2 \%$. This is the region where tunneling should be most visible. In comparison the right panel is based on trajectories in which the projectile easily passes over the barrier since its incidence energy amounts to more than three times the barrier height. Comparing the two isotopes, they behave very similar in both panels. D exhibits less distortion than $\mathrm{H}$ just because the spring constant between the beads is proportional to the atomic mass. The general shape of the curves is also remarkably similar. As the projectiles approach the barrier they are first slightly compressed in the $z$-direction and then expand as they reach the barrier top. Behind the barrier they again contract which is the normal behavior of RPs when transitioning from a free to a bound state.

The initial slight compression before the barrier can be understood by the spacial extension of the RP. Some beads encounter the barrier earlier than others. Hence, the beads up front are being slowed down by the repulsive potential while the ones furthest away from the surface are still traveling at their initial velocity. This leads to 
a spacial compression in the direction of travel which at least contains a component in the $z$-dimension. The same argumentation holds for the maximum of $r_{z}$ : some beads are accelerated towards the surface by forming the $\mathrm{C}-\mathrm{H}$ bond while other still need to overcome the barrier. This extends the RP and reaches its maximum when its center of mass coincides with the barrier top. In the left panel this moment occurs at approximately $1.8 \AA$ for both isotopes. There is no difference visible between them because the projectiles approach the surface so slowly that the $\mathrm{C}$ atoms have enough time to relax. This is not the case in the right panel where $r_{z}$ of $\mathrm{H}$ peaks closer to the surface because the lighter isotope moves faster.

In conclusion, neither the ZPE at the transition state, nor tunneling through the barrier has a significant impact on the sticking probability at $1 \mathrm{eV}$. More sophisticated methods might be necessary to fully confirm this finding. But given the fact that the sticking probability curves for $\mathrm{H}$ and $\mathrm{D}$ with and without NQEs nearly perfectly overlap and that the behavior of the gyration radius near and well above the adsorption threshold look so similar, it is unlikely that NQEs will turn out to have a large impact. In principle one would need to compare adsorption rates between classical transition state theory and for example the instanton approach to calculating rates [244] or some other method based on approximate quantum mechanics. These rates could subsequently be used to determine the tunneling factor which should be close to unity in case NQEs do not influence the adsorption process.

One thing left to discuss is panel b) of Fig. 51 where a clear difference between included and neglected NQEs is visible. One way to explain the effects is depicted in Fig. 53. First the higher sticking probability of $\mathrm{D}$ atoms can be explained by inertial effects. In the classical and RPMD case D atoms find much larger areas to stick on the surface compared to $\mathrm{H}$ atoms. The only surface site where sticking is prohibited is if they aim at the center of a ring. As soon as they aim somewhere near a carbon atom they get caught and a permanent $\mathrm{C}-\mathrm{H}$ bond is formed.

Switching on NQEs decreases the sticking probability of both isotopes. $\mathrm{H}$ is more affected than D which can be better seen in Fig. 51 over a range of normal incidence energies. Still, the sticking probabilities can be compared by simply looking at the brightness of each panel in Fig. 53 and it serves a different main purpose anyway. Comparing $\mathrm{H}$ atom sticking in panels a) and c) the repulsive area in the ring center grows when including NQEs. This is especially evident above and below the horizontal dividing line between the panels which would serve as a mirror if NQEs did not have any effect. Additionally in panel c) the bright spots next to carbon atoms appear dimmed compared to the classical simulation. It looks as if the contour di- 
a) classical $\mathrm{H}$

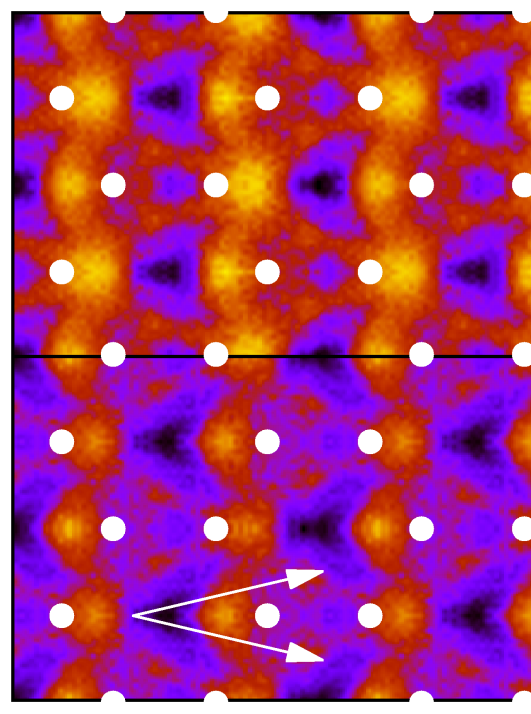

c) RPMD H b) classical D

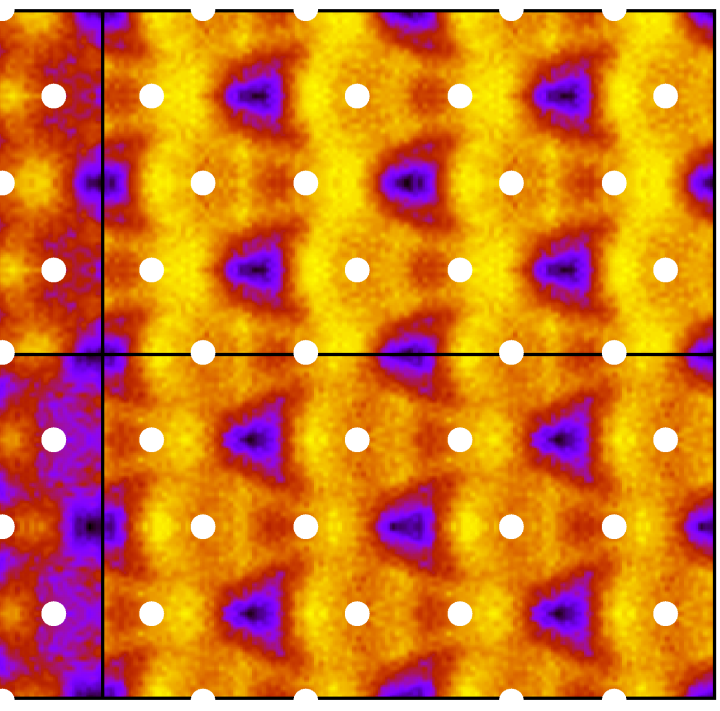

d) RPMD D
$P($ stick $) / \%$

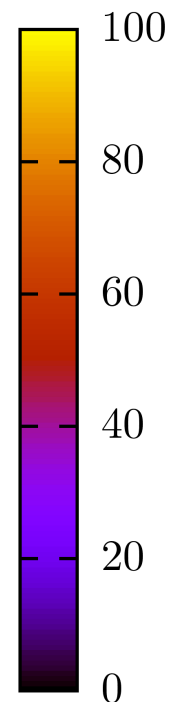

0

Figure 53: Sticking probability of $\mathrm{H}$ and $\mathrm{D}$ atoms on free-standing graphene at $300 \mathrm{~K}$ with and without the inclusion of NQEs. The incidence energy is $E_{\mathrm{i}}(\mathrm{H})=1.92 \mathrm{eV}, E_{\mathrm{i}}(\mathrm{D})=1.87 \mathrm{eV}, \theta_{\mathrm{i}}=40^{\circ}$ and $\varphi_{\mathrm{i}}=\mathcal{N}\left( \pm 13.5^{\circ} ; 2.12^{\circ}\right)$ with respect to a $\mathrm{C}-\mathrm{C}$ bond as indicated by the white arrows in panel c). These points correspond to $E_{\mathrm{n}}=1.1 \mathrm{eV}$ in Fig. 51. Carbon atoms are shown as white dots and the contour dimension is based on the aiming point of the projectiles $1 \AA$ above the surface plane. This is approximately as close as the projectiles that stick approach the surface plane at the given incidence conditions. Therefore, this heat map shows the particle's catchment area in dependence on the isotope and whether or not NQEs are considered.

mension was given a different range in this panel which means the surface becomes overall more repulsive when NQEs are considered.

In a plot of average energy loss versus normal incidence energy the two curves of $\mathrm{H}$ atom scattering at $E_{\mathrm{i}}=1.92 \mathrm{eV}$ with and without NQEs yield a hysteresisresembling shape shown in Fig. 54. At low and high incidence angles the curves overlap, but they deviate from one another in the middle. In this region, average energy losses based on RPMD calculations are in fact higher. Intuitively, one would assume that a higher energy loss indicates more energy transfer and thus a higher sticking probability. But here the important process that determines or rather inhibits sticking is the ability to escape from the surface after collision. Because NQEs lead to less energy transfer compared to classical simulations the sticking probability is lower. In consequence some projectiles that would classically stick to the surface are able to escape. Of course these barely escaping particles show up at the high energy loss end of the distribution thereby increasing the average energy loss.

Another approach to why NQEs can lead to a higher average energy loss is to 
Figure 54: Simulated total average energy loss of $\mathrm{H}$ atoms scattering from free-standing graphene. Their incidence energy is $E_{\mathrm{i}}=1.92 \mathrm{eV}$, $\varphi_{\mathrm{i}}=\mathcal{N}\left(13.5^{\circ} ; 2.12^{\circ}\right)$ and the surface is at $300 \mathrm{~K}$. Different normal energies were achieved by varying the polar incidence angle from $0^{\circ}$ to $70^{\circ}$. The scattered projectiles arrive with less kinetic energy at the all-seeing detector in the medium $E_{\mathrm{n}}$-range if NQEs are taken into account.

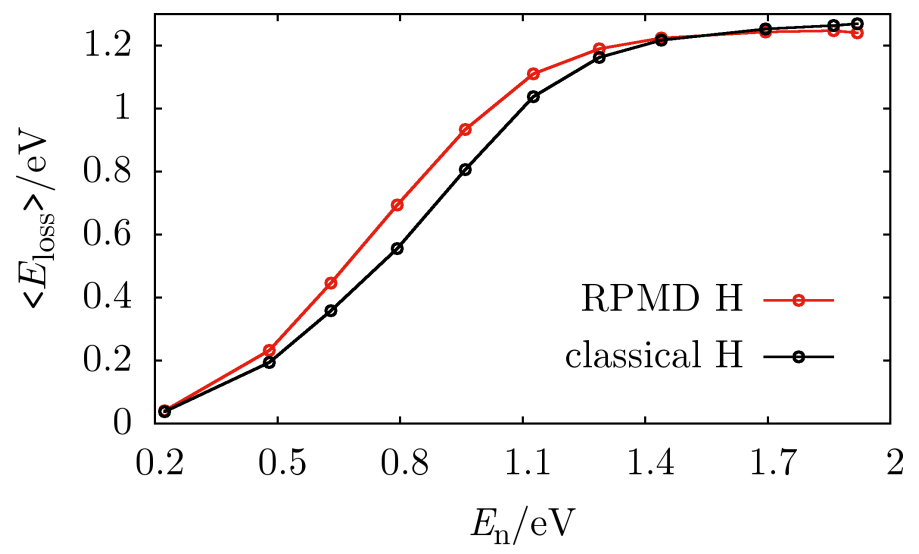

imagine an inversion of the contour dimension of Fig. 53. This would yield a map of where atoms that scatter come from, but it would not necessarily reveal how much energy they lost. Qualitatively speaking, however, projectiles scattering from the ring center where they find a purely repulsive wall cannot lose much of their energy. If they hit close to $\mathrm{C}$ atom but escape nevertheless, there is a high probability of a large energy loss. This last case is more probable when using RPMD instead of classical dynamics hence, the larger average energy loss.

The reason for this behavior can be found in the ZPE of the $\mathrm{C}-\mathrm{H}$ bond. The distortion of the carbon lattice which takes up a large part of the dissipated kinetic energy is the same with and without NQEs because temperature, incidence energy and all other parameters are the same. Independent of how much energy the $\mathrm{H}$ atom loses during the collision it is clear a shallower chemisorption well makes it easier for the particle to escape. The ZPE of the $\mathrm{C}-\mathrm{H}$ bond amounts to $305 \mathrm{meV}$ which is similar to the barrier height to adsorption. It stands to reason that such value has a much larger effect on the dynamics than ZPE at the transition state which is an order of magnitude smaller. Tunneling through the barrier is not an important escape mechanism. It was shown to be insignificant for the adsorption process at low energy and conversely it is of no importance for enabling barrier re-crossing. Tunneling through an asymmetric barrier is equally efficient no matter from what site the barrier is being approached [245].

\subsubsection{Substrate Effect}

Inching closer towards experimental conditions the influence of a weakly interacting substrate on the sticking probability shall be analyzed in this subsection. In experiment epitaxial graphene is created by chemical vapor deposition of ethylene on a clean Pt(111) surface. The procedure is described in detail in Ref. [239]. Graphene 


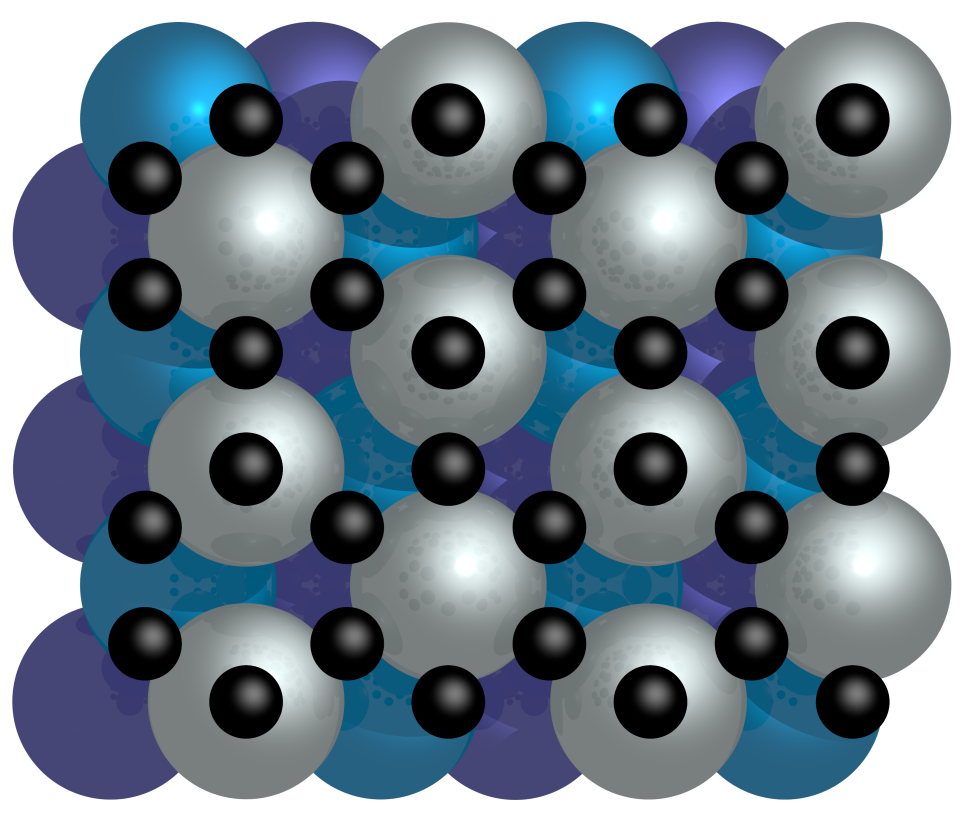

Figure 55: Top view of the simulation cell used to quantify the influence of the substrate on the $\mathrm{H}$ atom sticking probability on graphene. A single graphene layer is placed on a $\mathrm{Pt}(111)$ surface consisting of four atomic layers. The bottom two layers are held fixed to prohibit center of mass movement. The substrate is ABC-stacked as shown in Fig. 7. Gray spheres show the first platinum layer, the second one is metallic blue and the third one is indicated by purple spheres. Positions of the carbon atoms are marked by black spheres. The cell geometry was adopted from Ref. [256].

only weakly interacts with the platinum surface since it is only bound by dispersive interactions. This has been confirmed experimentally by micro-ARPES measurements [246] and using the meta-GGA [247], as well as the LDA and GGA [248] levels of theory. It barely affects phonon dispersion [249, 250] and elastic properties [251] of graphene. The interaction strength per carbon atom is estimated by Hamada and Otani to be $68 \mathrm{meV}$ at $3.24 \AA$ separation [252] based on the vDW-DF2 ${ }^{\mathrm{C} 09_{\mathrm{x}}}$ functional [253]. This functional together with the second version of vdW-DF [254] was found to currently be the best combination to describe graphene on metals [252]. This was confirmed by Toyoda et al. who arrived at a binding energy of $74 \mathrm{meV}$ also using vdW-DF2 with C09, but a slightly different setup [255].

Intuitively one would expect the Pt substrate to have an effect on the absorption energetics. Especially because a radical is formed in the adsorption process which is transfered to the extended $\pi$-electron system pointing exactly towards the metal. It can then easily affiliate itself with the surface electronic states. However, the cited experiments show that the opposite is the case and that the graphene layer is quasi free-standing. From the molecular dynamics point of view this is very fortunate. There exists no single global PES which can describe the interaction between $H$, graphene and platinum sufficiently accurate. But since graphene and platinum are only weakly interacting I could use a superposition of several different PESs. The Pt$\mathrm{Pt}$ and $\mathrm{H}-\mathrm{Pt}$ interaction can be accurately described using EMT with the optimized parameters listed in Tab. 6. REBO can be used for the $\mathrm{C}-\mathrm{C}$ and $\mathrm{C}-\mathrm{H}$ interaction and the graphene layer can be bound to the substrate by a Lennard-Jones potential. 


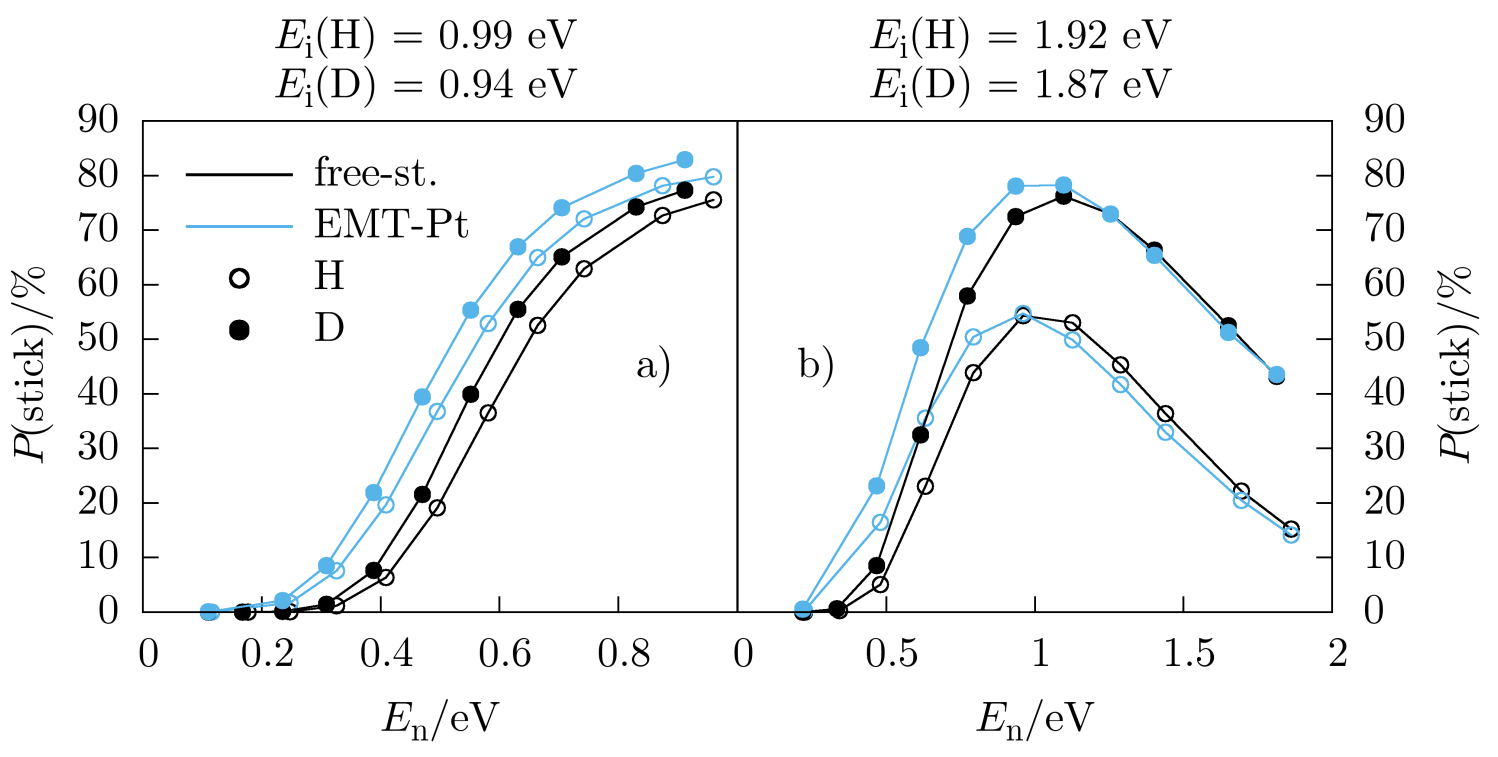

Figure 56: Sticking probability of $\mathrm{H}$ (open circles) and $\mathrm{D}$ atoms (filled circles) at $1 \mathrm{eV}$ shown in panel a) and $2 \mathrm{eV}$ in panel b). Black curves describe the probability to adsorb on free-standing graphene and blue curves show the influence of a $\mathrm{Pt}(111)$ substrate only bound to graphene via dispersive forces modeled by a Lennard-Jones potential. The substrate leads to a shift towards lower normal incidence energies in panel a). The same shift can be observed in the right panel up to $E_{\mathrm{n}}=1 \mathrm{eV}$. At higher energies the substrate only causes minor differences in the sticking probabilities.

The LJ parameters I employ are $\sigma=3.35 \AA$ and $\varepsilon=60 \mathrm{~K}$ which is approximately $5 \mathrm{meV}$. The value of $\sigma$ may seem a bit too large since it would lead to a $\mathrm{C}-\mathrm{Pt}$ equilibrium distance of $\sqrt[6]{2} \sigma=3.76 \AA$. One has to keep in mind though that the LJ potential describes a long-range interaction. In the actual simulation the second and third Pt layer also pull on the graphene sheet which in the end brings the CPt distance down to $3.3 \AA$ and more in line with literature values. The same long range interaction is also responsible for the very small value of $\varepsilon$. There are many Pt-atoms that each carbon is interacting with. Thus by adding up all contributions one arrives at a per carbon atom binding energy of $70 \mathrm{meV}$. The simulation cell structure is adopted from Ref. [256] and is shown in Fig. 55. The advantage of this setup is that the Pt lattice constant only needs to be reduced by $2 \%$ so that one Pt$\mathrm{Pt}$ bond matches the length of two $\mathrm{C}-\mathrm{C}$ bonds. This is as close as one can get to the geometry of the real system when planning to do extensive scattering simulations.

The sticking probability of $\mathrm{H}$ and $\mathrm{D}$ atoms on graphene with a $\mathrm{Pt}(111)$ substrate is shown in Fig. 56. The results for free-standing graphene are provided as a reference. The sticking curves in panel a) showing $1 \mathrm{eV}$ incidence energy projectiles are basically shifted about $80 \mathrm{meV}$ towards lower normal energies compared to the free-standing 


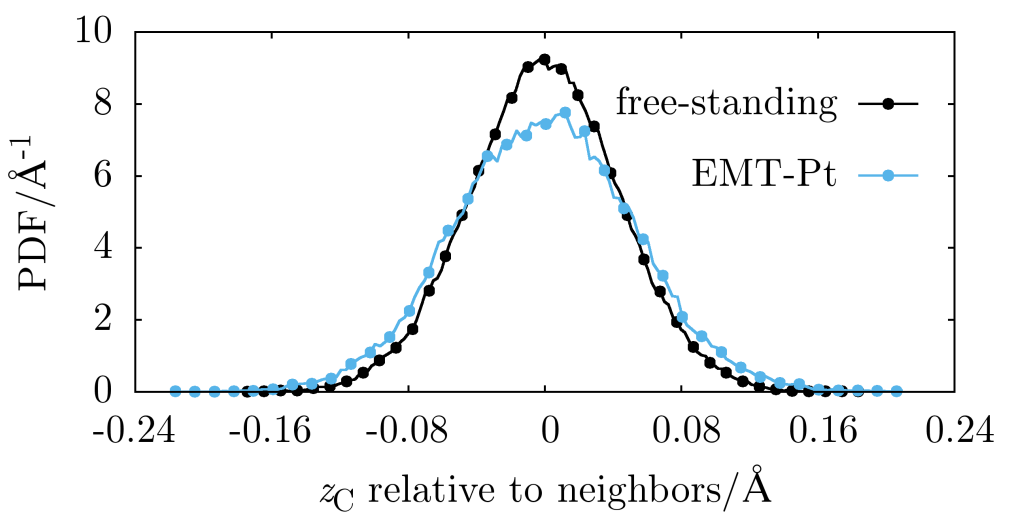

Figure 57: Probability density function of each carbon atom's $z$-position in comparison to the average $z$ position of its three nearest neighbors. The distribution including the substrate is broader and asymmetric indicating an increased motion in the $z$-coordinate that facilitates sticking of an impinging particle.

surface. This observation is independent of the isotope; both of them show the same behavior. The same trend with about the same shift is also apparent in panel b) up to $1 \mathrm{eV}$. At normal incidence energies above that value the sticking probabilities with and without the substrate are about equal. One might argue that the shift is due to the C-Pt binding energy that amounts to $70 \mathrm{meV}$ and is simply being passed on to the $\mathrm{C}-\mathrm{H}$ interaction. But this argumentation lacks any physical motivation. The $\mathrm{C}-\mathrm{H}$ interaction is not influenced by the platinum surface because it is not part of the REBO potential. Though there is an attractive interaction between $\mathrm{H}$ and Pt stemming from the EMT potential, at the barrier region about $1.7 \AA$ above the graphene surface, the H-Pt distance is roughly $5 \AA$. Looking at Fig. 15 the H-metal interaction starts at approximately one metal inter-layer distance above the surface. In the case of platinum this happens to be at about $2.3 \AA$ above the Pt surface. But this would be $1 \AA$ below the graphene layer in my setup. Therefore, this cannot be the reason for the observed shift in the sticking probability curves.

It turns out the C-Pt interaction is responsible for the change in the sticking probability curves, but not by influencing the $\mathrm{H}$ atom directly. Rather the additional interaction in the $z$-direction alters how the carbon atoms in the graphene behave. By adding the Pt substrate the graphene system is transformed from a $2 \mathrm{D}$ into a 3D system. There are now atoms weakly, but consistently pushing and pulling on the graphene which leads to an increased motion in the $z$-direction. Also if a cut through the PES like Fig. 45 is calculated, the barrier towards adsorption is reduced by $60 \mathrm{meV}$. With this in mind it becomes more probable, simply due to thermal fluctuations, that the targeted $\mathrm{C}$ atom assumes a geometry that facilitates sticking. This is shown in Fig. 57. It shows the probability density of how much above or below its three neighbors every carbon atom is located. These results come from simulations of only the surface at $300 \mathrm{~K}$. For each carbon atom in each time step its 
three nearest neighbors are found and their average $z$-position is calculated. Then the difference in $z$ to the central $\mathrm{C}$ atom is recorded and finally all of them are histogrammed.

In the case of free-standing graphene the distribution resembles a Gaussian. This makes sense because without a substrate it can be expected that small deflections in $z$ follow a harmonic oscillator potential. With the Pt substrate the distribution becomes broader and asymmetric. The asymmetry can be explained by a superposition of the harmonic carbon network potential with the anharmonic LJ potential. Although this is a very weak interaction the influence on the probability density is clearly visible. The broadening is due to platinum atom motion. Since the top two Pt layers are also moving the minimum of the LJ potential depends on their positions which increases the average deflection of the $\mathrm{C}$ atoms. Note that Fig. 57 does not show the distribution of $z$-coordinates, but the distribution of a geometrical arrangement that favors sticking if $z_{\mathrm{C}}$ relative to its neighbors is positive. The substrate mainly reduces the probability of graphene being flat. Hence, the increased probability of $\mathrm{H}$ finding a nonplanar surface is responsible in the end for the observed changes in the sticking probability up to $E_{\mathrm{n}}=1 \mathrm{eV}$ when adding a $\operatorname{Pt}(111)$ substrate.

Above $1 \mathrm{eV}$ the shape of the curves is determined by the ability of the particle to break the transient $\mathrm{C}-\mathrm{H}$ bond. The binding energy curve is given by the REBO PES whose absolute energies are at least an order of magnitude larger than those of the LJ potential. Therefore, the escape pathway remains unchanged for the most part which leads to an overlap of the curves at higher normal energies.

\subsubsection{Comparison with Experiment}

In experiment of course the surface and graphene layer are not as nicely ordered as in my simulation cell. The C-Pt bond is simply not strong enough to form a specific angle between the graphene layer and the substrate. There are two rotational graphene domains at an approximately equal abundance which are rotated by $27^{\circ}$ with respect to one another. Each domain in itself has a spread of roughly $5^{\circ}$ full width at half maximum. This was also the motivation for me to perform most of the simulations at an incidence azimuthal angle of $\varphi_{\mathrm{i}}=13.5^{\circ}$ with respect to a C-C bond. Simply because in experiment the projectiles were also launched right between the two principal domain directions. The spread of the domains is modeled by an azimuthal incidence angle normal distribution with $\mu=13.5^{\circ}$ and $\sigma=2.12^{\circ}$. In 


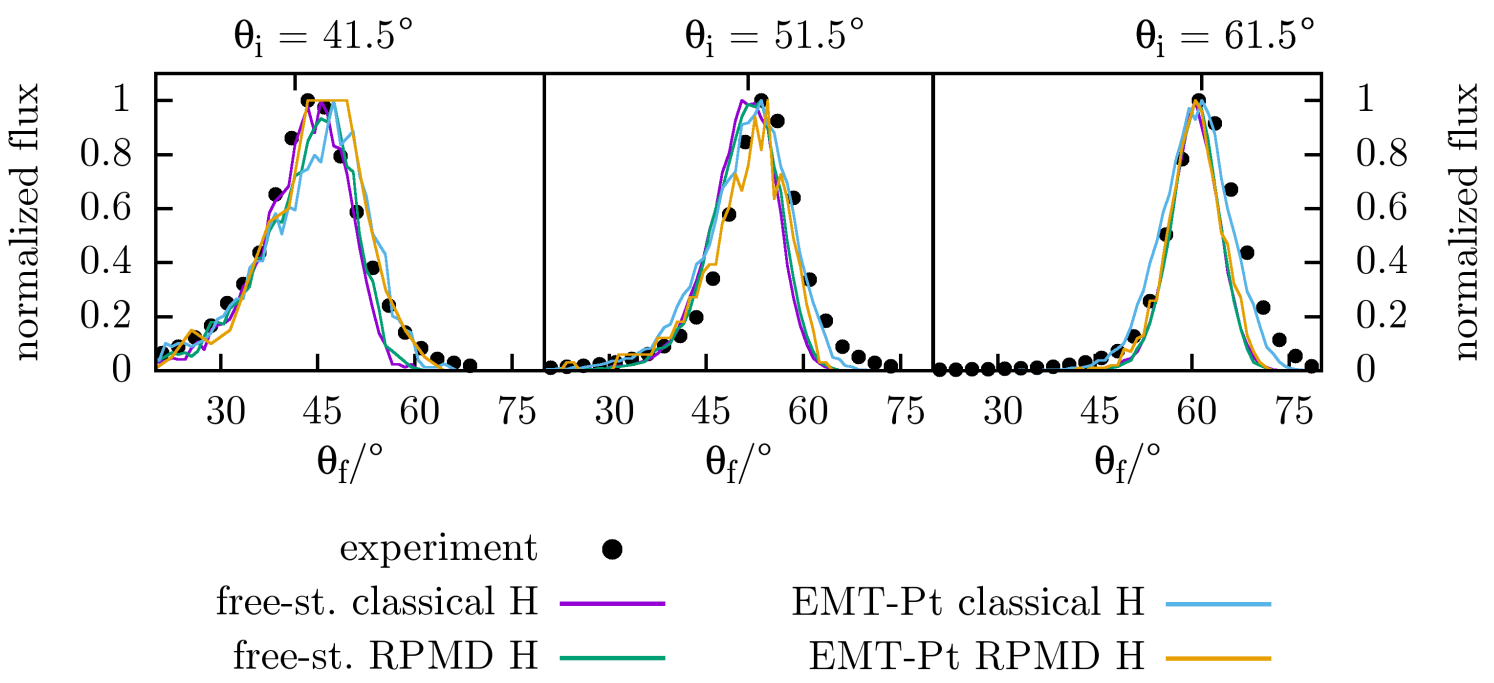

Figure 58: Angular distribution of $\mathrm{H}$ atom in-plane flux after scattering from graphene. The abscissa denotes the scattering polar angle $\theta_{\mathrm{f}}$, and the ordinate indicates the normalized flux. Black dots show experimentally obtained data and the colored lines indicate different setups to modeling the scattering process. The incidence polar angle $\theta_{\mathrm{i}}$ is displayed above each panel. All flux data was collected with an in-plane detector having a diameter of $2.9^{\circ}$ solid angle. The surface is at $300 \mathrm{~K}$ and the incident particles are provided with a kinetic energy of $0.99 \mathrm{eV}$. The graphene rotational domain structure is modeled by $\varphi_{\mathrm{i}}=\mathcal{N}\left(13.5^{\circ} ; 2.12^{\circ}\right)$.

contrast to experiment it is much easier to vary $\varphi_{\mathrm{i}}$ and the position of the detector in the simulations. The relative orientations are in the end the same as in experiment.

Gao et al. studied structural properties of epitaxial graphene grown on $\operatorname{Pt}(111)$ [257]. They found a flat graphene layer with distortions of less than $0.3 \AA$ in the $z$-direction. The $2 \times 2$ Moiré superstructure shown in Fig. 55 can actually exist, but $3 \times 3,4 \times 4,(\sqrt{37} \times \sqrt{37}) \mathrm{R} 21^{\circ},(\sqrt{61} \times \sqrt{61}) \mathrm{R} 26^{\circ}$ and $(\sqrt{67} \times \sqrt{67}) \mathrm{R} 12^{\circ}$ have also been reported. What Moiré structure is formed depends on the nucleation rate during dosing of ethane on the platinum surface at an elevated temperature. Epitaxial graphene forms extended domains with usual sizes of tens of nanometers and it has a low defect density. This is an important finding since otherwise the simulation cell would not be a valid model of the real system. In the experiment to which my simulations will be compared there exists most likely a $4 \times 4$ Moiré structure.

The challenge is that in experiment the detector can only be rotated in-plane. Thus any atoms that have their azimuthal angle changed by more than $1.45^{\circ}$ during the collision cannot be detected. In order to obtain the sticking probability experimentally, Hongyan Jiang assumes a Gaussian line shape of the scattering signal in his experiment [6]. For $E_{\mathrm{i}}=1 \mathrm{eV}$ this seems to be a good approximation as shown in Fig. 58. The black dots form a Gaussian at high polar angles which becomes 


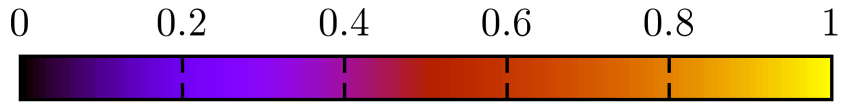

arb. units
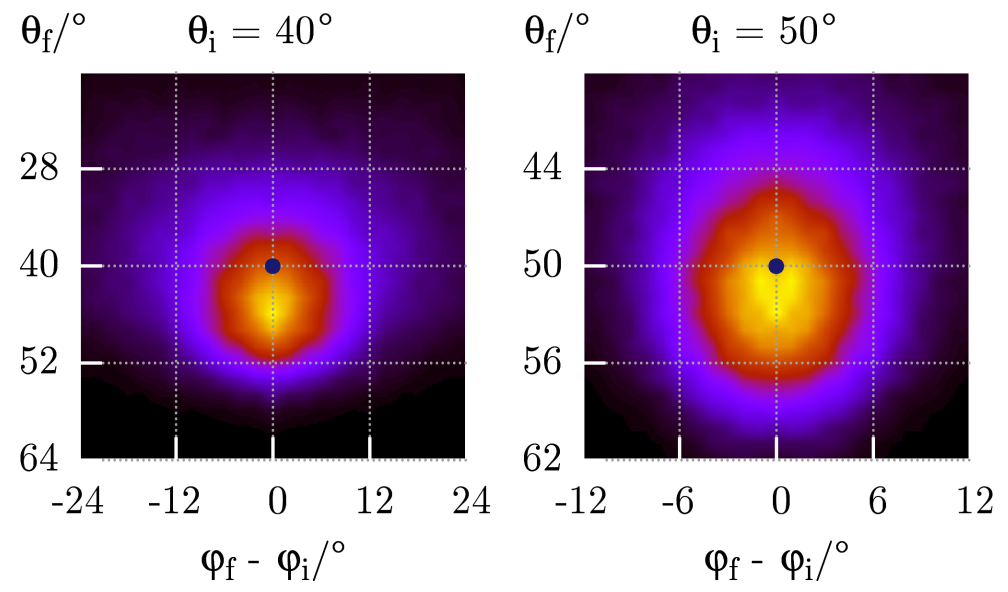

Figure 59: Heat map of the simulated scattering signal of $\mathrm{H}$ atoms at $E_{\mathrm{i}}=0.99 \mathrm{eV}$. Data stems from classical trajectory simulations utilizing a free-standing graphene surface at $300 \mathrm{~K} . \varphi_{\mathrm{i}}$ respects rotational domains and the panels show the normalized flux at different $\theta_{\mathrm{i}}$ which is indicated above each one. The $x$ - and $y$-axis quantify the spread of the scattering signal in terms of $\varphi_{\mathrm{f}}$ and $\theta_{\mathrm{f}}$, respectively. Dotted grid lines help to assess the cylindrical symmetry of the flux since both axes have the same range in the square panels. The blue dot in the center of each one marks the specular scattering direction.

more and more skewed at smaller polar incidence angles. Regardless of what theoretical setup is used the curves including skew can be well reproduced. Neither the substrate nor NQEs have any discernible influence on the results. This is different from the sticking probability at $E_{\mathrm{i}}=1 \mathrm{eV}$ on which the substrate has a substantial effect. Another challenge is that there are no absolute numbers in experiment. The number of $\mathrm{H}$ atoms per pulse can only roughly be estimated and there is no way of obtaining absolute counts at the detector.

The idea to arrive at a sticking probability anyway is the following: the in-plane $\mathrm{H}$ atom flux at $E_{\mathrm{i}}=1 \mathrm{eV}$ is measured at various incidence angles from $31.5^{\circ}$ to $65.5^{\circ}$. A Gaussian is subsequently fitted to each of the obtained curves. Assuming cylindrical symmetry the volume $V$ under a 2D Gaussian can be calculated by $V=2 \pi A \sigma^{2}$ with $A$ being proportional to the intensity and $\sigma$ is the standard deviation measured inplane. These volumes are still arbitrary in terms of absolute numbers which requires another approximation. At the five highest incidence angles it can be expected that no or only very few projectiles stick to the surface. This assumption is supported by theory and also the calculated volumes are about equal. Therefore, these points can be used as reference data representing zero sticking. If the ratio between a calculated volume at some $\theta_{\mathrm{i}}$ and these reference points is smaller than one, the signal drop 


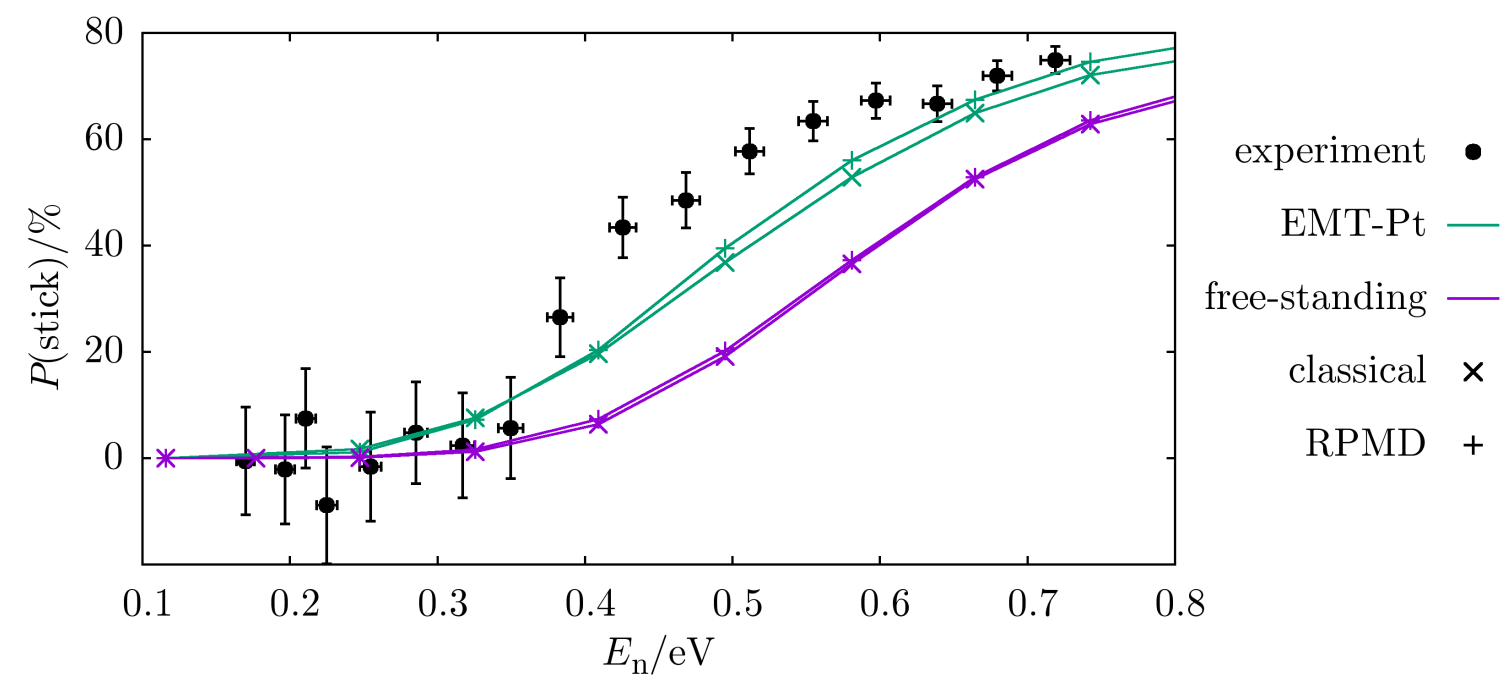

Figure 60: Sticking probabilities of $\mathrm{H}$ atoms on graphene at $E_{\mathrm{i}}=0.99 \mathrm{eV}$. The polar angle was varied to arrive at different normal incidence energies in theory and experiment. Black dots represent adsorption probabilities derived from the cylindrical symmetry assumption based on inplane flux measurements. Error bars reflect estimated uncertainties in the measured flux. The purple curve shows sticking probabilities of $\mathrm{H}$ atoms scattered from free-standing graphene. As shown in Fig. 51 the inclusion of NQEs does not noticeably change the results. The green curve depicts the adsorption probability of $\mathrm{H}$ on graphene with the $\mathrm{Pt}(111)$ substrate. Here NQEs enhance sticking ever so slightly. The additional LJ interaction also leads to a better agreement with experiment.

can be attributed to sticking.

Projectiles can be categorized according to three scenarios. They either stick, scatter out-of-plane, or scatter in-plane. Since the out-of-plane distribution has about the same width as the in-plane distribution and this is accounted for by applying cylindrical symmetry any additional loss of signal has to be due to sticking. Of course there is no experimentally accessible out-of-plane data, but theory predicts a fairly similar distribution in both planes if no transient $\mathrm{C}-\mathrm{H}$ bond is formed during collision. This is shown in Fig. 59 for $\mathrm{H}$ scattering from a free-standing graphene surface. Note that the point of highest intensity deviates more and more from the specular angle as the incidence polar angle decreases. This is only a small effect, but also visible in Fig. 58 .

The tendency towards super-specular scattering and the skew of the distribution are the two largest sources of error in the cylindrical symmetry assumption. Since it ignores the heavy tail of the signal in the direction of smaller polar scattering angles it yields an upper limit of the adsorption probability. This is because the scattered signal that the rotated Gaussian does not capture is attributed to sticking. But in fact any missing signal can be due to multiple reasons namely a non-Gaussian 


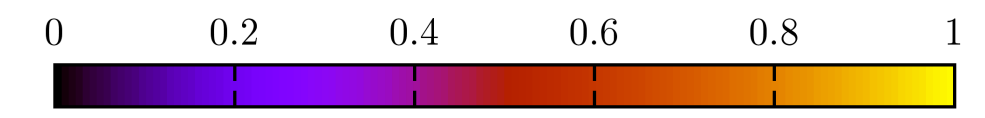

arb. units
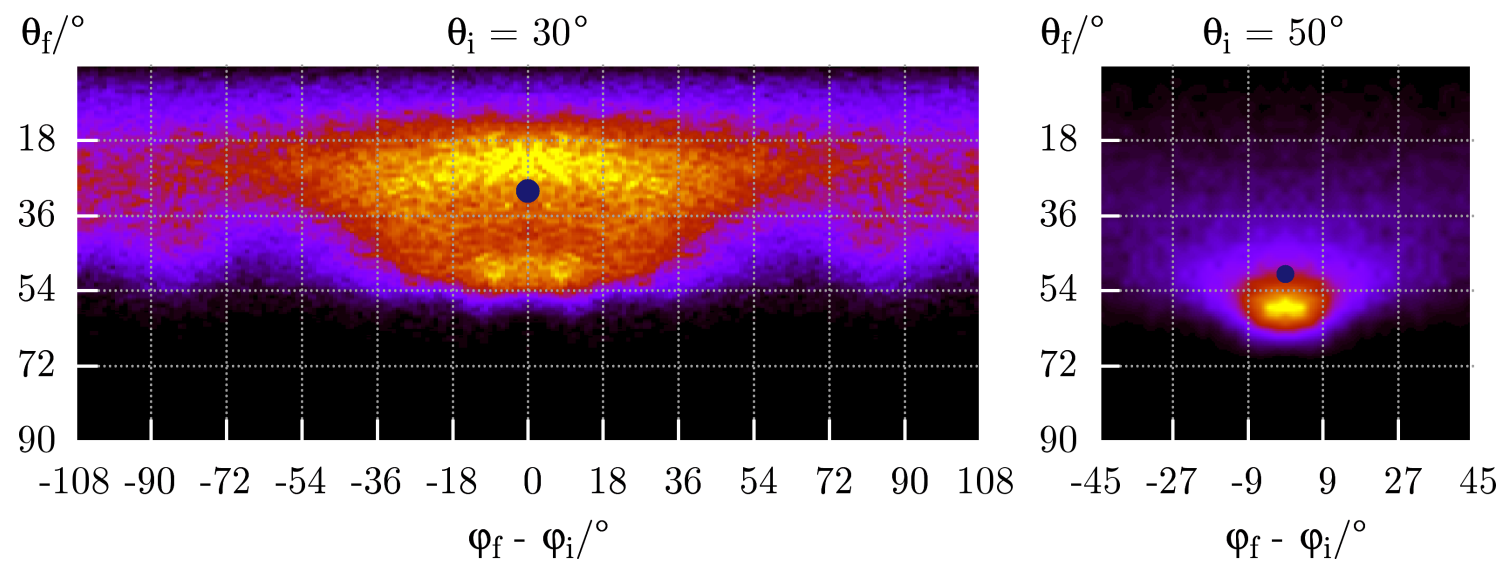

Figure 61: Normalized scattering intensity based on azimuthal angle deviation $\varphi_{\mathrm{f}}-\varphi_{\mathrm{i}}$ and polar scattering angle $\theta_{\mathrm{i}}$. The free-standing graphene in these simulations has a temperature of $300 \mathrm{~K}$ and the incident particles are provided with $E_{\mathrm{i}}=1.92 \mathrm{eV}$. The simulations include the described two-domain structure of the surface. In the left panel showing results obtained with $\theta_{\mathrm{i}}=30^{\circ}$ a very broad feature is visible that has a bigger spread in the azimuthal dimension than in the polar one. The highest intensity can still be found in the forward scattering direction near the specular angle indicated by a blue dot. The left panel shows results produced at $\theta_{\mathrm{i}}=50^{\circ}$. The shape of the scattered signal was circular when $1 \mathrm{eV} \mathrm{H}$ atoms were used. But at nearly twice the incidence energy the signal spreads more in the azimuthal direction and a heavy tail towards smaller polar angles appears. Both panels are scaled such that $1^{\circ}$ in $\theta$ and $\varphi$ have the same lateral length.

in-plane distribution, an out-of-plane Gaussian having a width different from in the in-plane one, or true sticking.

A comparison of the experimentally obtained adsorption probability curve employing the cylindrical symmetry assumption is shown in Fig. 60. The same figure contains calculated sticking probability curves with and without NQEs on freestanding graphene and with the Pt(111) substrate. NQEs still do not have any significant effect on the sticking probability of $\mathrm{H}$ atoms at $1 \mathrm{eV}$ not even if a substrate is added. The experimentally and theoretically obtained curves both show a monotonously increasing adsorption probability in the given range of normal incidence energies. The onset of sticking is around $E_{\mathrm{n}}=0.3 \mathrm{eV}$ in theory and between 0.35 and $0.4 \mathrm{eV}$ in experiment. At higher $E_{\mathrm{n}}$ the experiment finds an adsorption probability that is consistently larger than any of the theoretical approaches. At the highest available normal energies all curves level off at $P$ (stick $)=70 \%$ to $80 \%$.

For projectiles at $2 \mathrm{eV}$ incidence energy unfortunately the sticking probability cannot be estimated from experiment. In this case the cylindrical symmetry assumption fails because there are atoms being scattered which have experienced a 
transient $\mathrm{C}-\mathrm{H}$ bond formation. At small incident normal energies graphene acts like a mirror and scatters particles quasi-elastically. Their energy and incidence angles remain basically unchanged. Provided with $2 \mathrm{eV}$, however, there are atoms joining the scattered fraction which have come in close contact with a $\mathrm{C}$ atom and can recross the barrier. Consequently their angular distribution is massively distorted. This can clearly be seen from the in-plane signal in experiment and is supported by theory. Heat maps of scattered flux from $\mathrm{H}$ atom scattering simulations at $1.92 \mathrm{eV}$ and two incidence polar angles are shown in Fig. 61.

Already at $\theta_{\mathrm{i}}=50^{\circ}$ a clear broadening in the out-of-plane direction is visible together with an extended tail towards smaller polar scattering angles. Due to the increased incidence energy it can probe more of the repulsive parts of the PES which leads to stronger deflections compared to the $1 \mathrm{eV}$ data at the same incidence angle. At $\theta_{\mathrm{i}}=30^{\circ}$ there is a very broad feature in the forward scattering direction. This shows how much a single collision with the surface along with transient bond formation and directional forces can deflect the impinging particle from its original path. Also note that quasi-elastically scattered atoms appear super-specular whereas those that underwent transient $\mathrm{C}-\mathrm{H}$ bond formation have their maximum intensity at a sub-specular angle. This is an immediate consequence of the direction that the $\mathrm{C}-\mathrm{H}$ bond points at which is parallel to the surface normal. Clearly in both cases shown in Fig. 61 it is impossible to infer the out-of-plane distribution if only data corresponding to $\varphi_{\mathrm{f}}-\varphi_{\mathrm{i}}= \pm 1.45^{\circ}$ is accessible.

Comparisons to trapping probabilities from the literature are fairly difficult to make because the incidence conditions can differ a lot. In the first experiment towards quantifying sticking probabilities conducted in 2002 Zecho et al. used thermal $\mathrm{H}$ and $\mathrm{D}$ atoms at $2000 \mathrm{~K}(\langle E\rangle=0.17 \mathrm{eV})$ as the impinging particles and found a sticking probability between $25 \%$ and $50 \%$ on a $150 \mathrm{~K}$ graphene surface [258]. From today's perspective these numbers seem a bit too high since it is known that there is at least a $0.25 \mathrm{eV}$ barrier to adsorption. Of course they did not use a monoenergetic beam and in a Maxwell-Boltzmann distribution there are atoms at higher kinetic energies. But on the other hand there exist also a lot of atoms with less than the mentioned $0.17 \mathrm{eV}$ which do not have any chance to adsorb.

Morisset et al. used mixed classical-quantum calculations of collinear scattering to assess the sticking probability [205]. In their approach the graphene phonons are treated classically while a one-dimensional quantum Gaussian wave packet method is used for the projectile approaching the surface. At a surface temperature of $300 \mathrm{~K}$ they found a nearly constant $\mathrm{H}$ atom sticking probability of $50 \%$ for incidence 
energies ranging from 0.2 to $1.1 \mathrm{eV}$. Their calculations also predict a $10 \%$ probability to adsorb at an incidence energy as low as $0.136 \mathrm{eV}$ which even increases to $40 \%$ if the surface is cooled to $10 \mathrm{~K}$. They attribute their findings to effective tunneling through the barrier and the fact that a cold surface does not have much energy in its phonons. Hence, it cannot transfer much to the projectile which means the energy transfer has to happen from the projectile to the surface leading to sticking. Unfortunately there is not a single comment in their paper about zero point energy effects.

Kerwin and Jackson used classical MD to look at sticking of $\mathrm{H}$ and D atoms on graphite [259]. They used the graphite lattice force field of Aizawa et al. [260] and came to similar conclusions as I did. The sticking probability curve with incidence energy on the $x$-axis has a single pronounced peak with a fat tail towards higher energies. The maximum of this distribution is a function of the barrier height which is why the blue curves in Fig. 56 peak at slightly lower normal incidence energies. They modeled the experiment of Zecho et al. and arrived at a sticking probability for $\mathrm{H}$ atoms of $6.6 \%$, but only stated that it was about the same for D atoms. Kerwin and Jackson mention in their paper that the experimentally determined high sticking probability is presumably due to secondary $\mathrm{H}$ atom absorption. In fact the barrier becomes much lower if the zero coverage limit is exceeded [261]. They also mention a private communication with Zecho saying that the D atom sticking probability in experiment is closer to $10 \%$ in the zero coverage limit, but apparently this result has never been published.

To better compare results obtained with the REBO-EMFT PES to published values I simulated the described experiment of the Zecho group. To arrive at a thermal projectile distribution the projectile velocity components are drawn from a Maxwell-Boltzmann distribution at $2000 \mathrm{~K}$. This automatically determines polar and azimuthal angle, as well as incidence energy. Note that in this simulation there are no approximations concerning the internal ring polymer modes because all $\mathrm{H}$ atoms are in thermal equilibrium. As in all other simulations a particle is stuck on the surface when its $z$-position at the end of the simulation is closer to the surface than its initial one. This is a very simple calculation. One only has to pay attention to the simulation time. There are many projectiles with large polar angles and thus large distances to travel because seen from the surface plane the probability density of polar incidence angles follows a cosine distribution. The calculated sticking probabilities for $\mathrm{H}$ and $\mathrm{D}$ atoms from classical and RPMD calculations with and without the substrate are summarized in Tab. 11. 
Table 11: Sticking probabilities in percent of $\mathrm{H}$ and $\mathrm{D}$ atoms on graphene with and without a $\operatorname{Pt}(111)$ substrate. The incidence energy and angles are distributed according to a MaxwellBoltzmann distribution of $2000 \mathrm{~K}$. This is what REBO-EMFT predicts to be the sticking probability in the experiment of Zecho et al. [258]. The $95 \%$ confidence interval is at most 0.07 percentage points.

\begin{tabular}{rcccc}
\hline \multirow{2}{*}{ surface } & \multicolumn{2}{c}{ classical } & \multicolumn{2}{c}{ RPMD } \\
& $\mathrm{H}$ & $\mathrm{D}$ & $\mathrm{H}$ & $\mathrm{D}$ \\
\hline free-standing & 1.27 & 1.30 & 1.53 & 1.52 \\
EMT-Pt & 2.36 & 2.30 & 2.76 & 2.72 \\
\hline
\end{tabular}

The biggest influence on the sticking probability is the presence of the substrate. With a Pt(111) surface below the graphene sheet the sticking probability nearly doubles. These small numbers can easily be influenced by the slightly smaller barrier and increased carbon atom motion perpendicular to the surface plane when the substrate is present. The simulation of NQEs also increases sticking. As already seen in the monoenergetic beam scattering simulations, tunneling through the barrier accelerates the reaction more than ZPE at the transition state slows it down. This is the true for both isotopes. There seems to be no inertial kinetic isotope effect in agreement with Kerwin's and Jackson's results. Overall, the sticking probabilities are smaller by a few percentage points compared to their results. The deviation is, however, well in line with the expected changes in sticking probability when using another high-dimensional PES for this system.

\subsection{Energy Loss Distribution}

The initial motivation to develop the described PESs was $\mathrm{H}$ atom scattering experiments performed by Hongyan Jiang. What he found is shown in panels a) to c) of Fig. 62. I note that some of the presented energy loss distributions have already been published in Ref. [6]. The polar axis indicates the polar scattering angle and the radial axis shows the fractional energy loss. The incidence angle is highlighted in red and all atoms have an incidence energy of $1.92 \mathrm{eV}$. At an incidence angle of $\theta_{\mathrm{i}}=30^{\circ}$ a broad angular distribution is observed with fractional energy losses ranging approximately from $25 \%$ to $75 \%$. At a higher incidence angle of $45^{\circ}$ a second component appears at a super-specular angle with only a very small energy loss. Atoms launched towards the surface at $\theta_{\mathrm{i}}=60^{\circ}$ are only scattered back quasielastically. This is also the case for particles incident with $E_{\mathrm{i}}=1 \mathrm{eV}$ regardless of 
0 0.2 0.4 0.6 0.8 arb. units
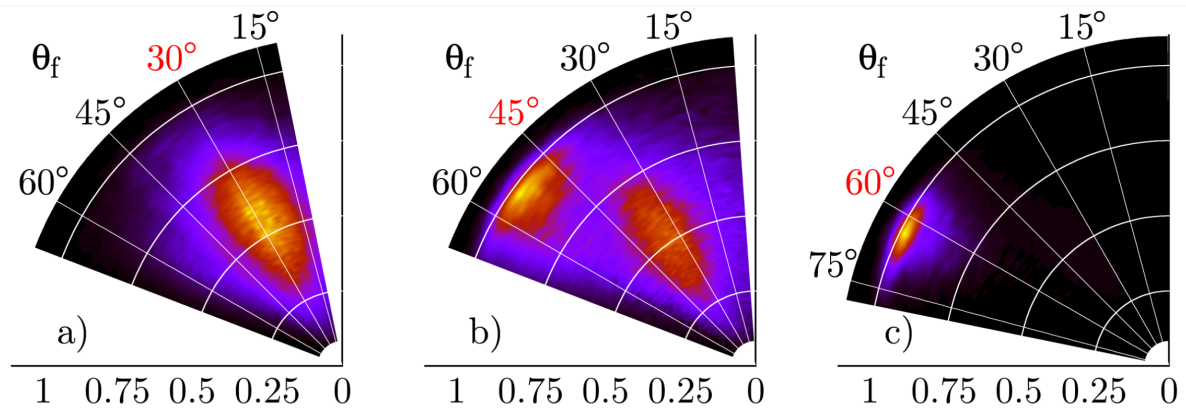

experiment

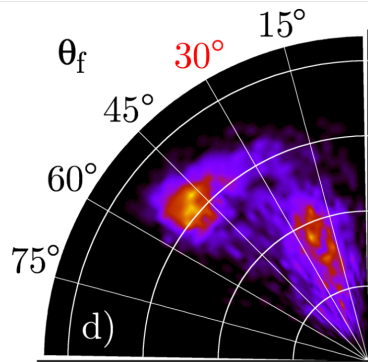

$\begin{array}{lllll}1 & 0.75 & 0.5 & 0.25 & 0\end{array}$

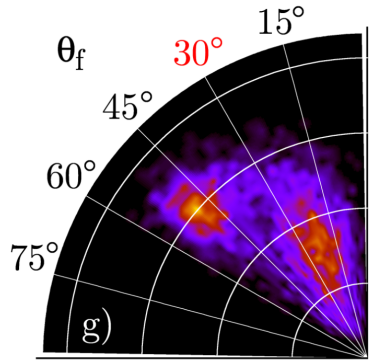

$\begin{array}{lllll}1 & 0.75 & 0.5 & 0.25 & 0\end{array}$ $E_{\mathrm{f}} / E_{\mathrm{i}}$

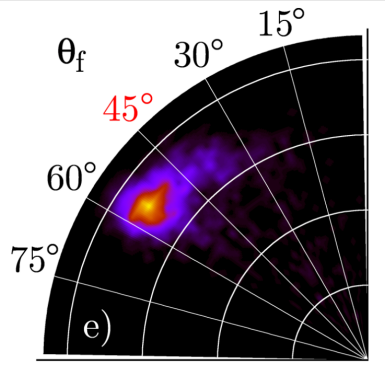

$\begin{array}{lllll}1 & 0.75 & 0.5 & 0.25 & 0\end{array}$

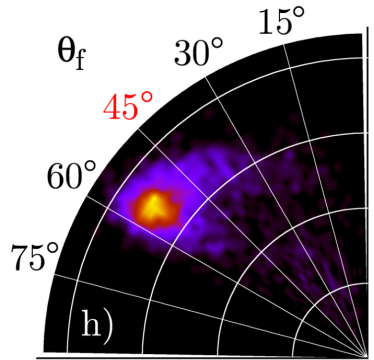

$\begin{array}{llllll}1 & 0.75 & 0.5 & 0.25 & 0\end{array}$

$E_{\mathrm{f}} / E_{\mathrm{i}}$

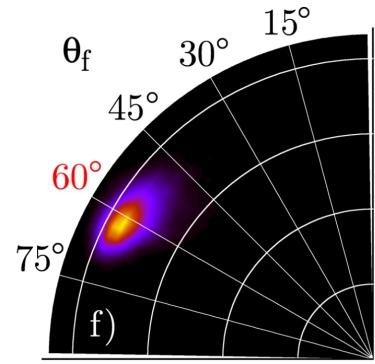

$\begin{array}{lllll}1 & 0.75 & 0.5 & 0.25 & 0\end{array}$

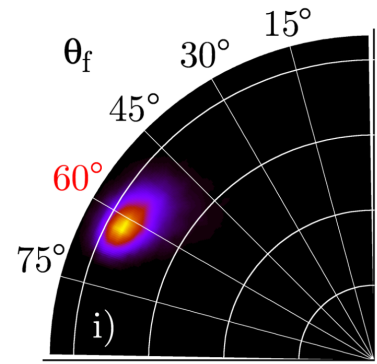

$\begin{array}{lllll}1 & 0.75 & 0.5 & 0.25 & 0\end{array}$

$E_{\mathrm{f}} / E_{\mathrm{i}}$
REBO-EMFT classical free-standing

REBO-EMFT RPMD

free-standing

Figure 62: In-plane retained energy and polar scattering angle distributions. The radial axis shows the fraction of retained energy and the polar axis indicates the scattering angle. Each row represents the results of one level of theory which is labeled to the site of it. The incidence polar angle stays the same in each column and is highlighted in red. The signals are scaled so that the maximum is illustrated by the same color in each panel.

$\theta_{\mathrm{i}}$. They all exhibit a localized signal at $\theta_{\mathrm{i}}=\theta_{\mathrm{f}}$ and can be found the supplementary information of the referenced paper. No matter what theoretical approach is used to model this reflective behavior of graphene at low incidence energy, all of them succeed.

Theory could help elucidate the involved mechanisms leading to these two very different energy loss behaviors. The fast channel appears due to atom scattering from 
purely repulsive parts of the surface. This includes the center of the six-membered carbon rings and depending on the incidence energy also the barrier to adsorption. I want to point out that any fast signal must not necessarily have been scattered from the barrier. In Fig. 62b) for example the normal energy component is close to $1 \mathrm{eV}$. Still, there is a one-to-one ratio of fast and slow signal. Ignoring all discussions about the barrier height for a moment, everyone agrees that particles incident with $E_{\mathrm{n}}=1 \mathrm{eV}$ plus the same parallel incidence energy will overcome that barrier to C-H bond formation at least once. Hence, the presence of the fast channel here proves that scattering from the center of the carbon ring also contributes to the part of the scattered signal and at this incidence condition is actually the only contributor.

Some atoms stick to the surface and some overcome the barrier twice. Only very rarely do $\mathrm{H}$ atoms experience multiple oscillations in the chemisorption well. The majority either sticks to the surface for much longer than the typical trajectory simulation time or is scattered back after a single collision. This gives rise to the slow component seen in the angle-resolved energy loss spectra. The interaction dynamics are described in detail in section 5.2 where it is explained how a large amount of energy can be transfered to the graphene sheet in a single collision on one electronically adiabatic PES.

Comparing the simulated energy loss distribution in panels d) to i) in Fig. 62 and all of Fig. 63 to the experimentally obtained ones, one notices the following details: at $\theta_{\mathrm{i}}=60^{\circ}$ there is only the fast channel present in experiment and in theory. In contrast to the sticking probability no real influence of quantum or substrate effects can be noticed. The reason is that at such a low normal incidence energy the particles are either elastically reflected from the surface or stick. And those that stick simply do not show up on these plots. The intermediate polar angle range around $\theta_{\mathrm{i}}=45^{\circ}$ where there are both components present in experiment is most difficult to model. An ever so slight mismatch of barrier heights or force constants either from an ab initio method or analytical PES leads to deviations from experimental observations. In principle all REBO-EMFT-based approaches neglect the slow component at this angle, but the energy loss and position of the fast peak is nearly on point. REBO-PBE, however, shows the exact opposite behavior. There is no fast component visible in the energy loss spectrum of panel Fig. 63h), but the polar and radial spread of the slow signal matches the experimental data.

At $\theta_{\mathrm{i}}=30^{\circ}$ REBO-EMFT erroneously still predicts some fast component whose intensity is diminished by adding the Pt-substrate. The slow part of the signal is by and large correctly modeled. The spread is somewhat smaller in theory than in ex- 


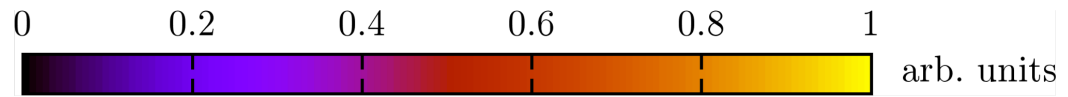
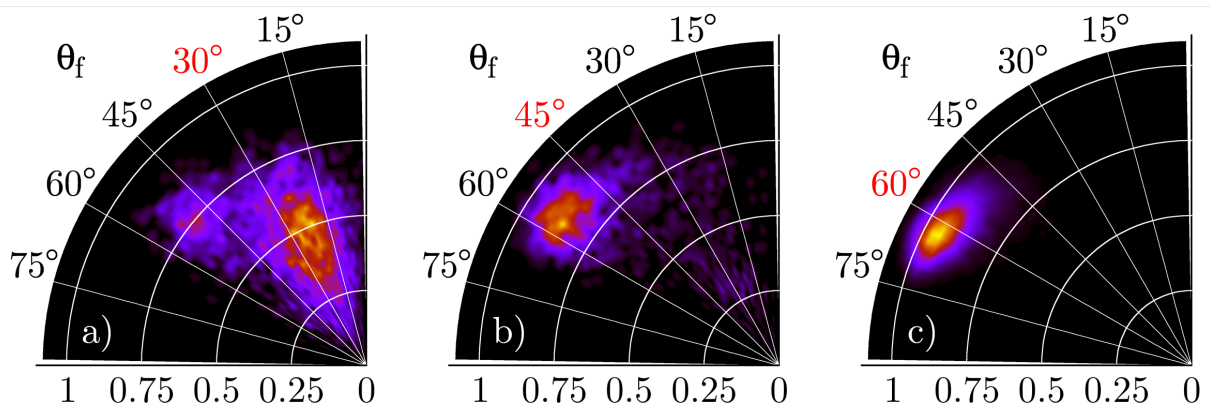

REBO-EMFT

classical

EMT-Pt
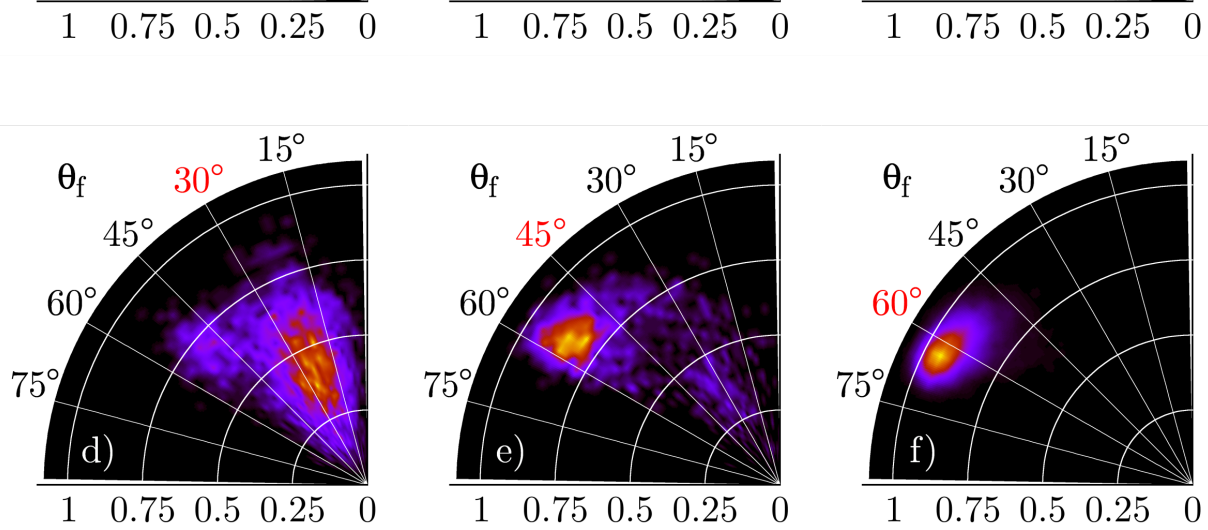

REBO-EMFT

RPMD

EMT-Pt
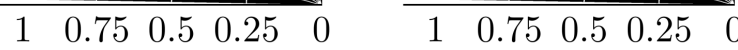

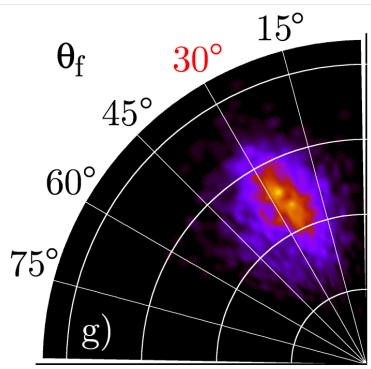

$\begin{array}{llllll}1 & 0.75 & 0.5 & 0.25 & 0\end{array}$

$E_{\mathrm{f}} / E_{\mathrm{i}}$

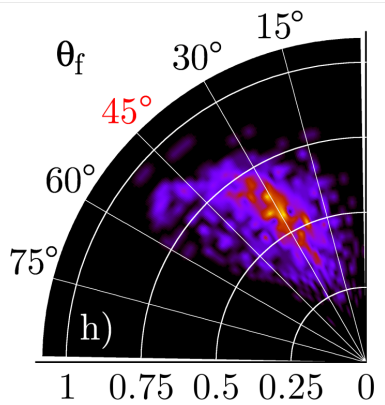

$E_{\mathrm{f}} / E_{\mathrm{i}}$

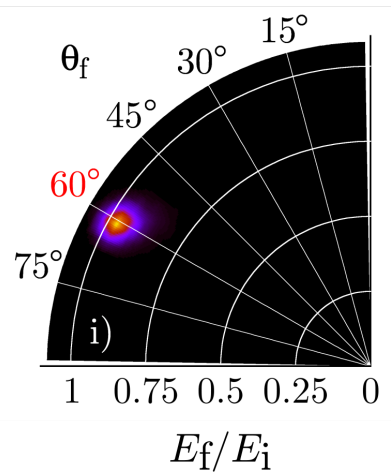

REBO-PBE

classical

free-standing

Figure 63: Same as Fig. 62 but results from other theoretical approaches are shown.

periment, but the main polar scattering direction and energy losses are reproduced. REBO-PBE accurately predicts only the slow channel, but the blob also appears too localized. In summary, it was shown that both parametrized PESs can qualitatively reproduce the experimental findings. Both have their shortcomings mainly because the incidence angle-dependent fast to slow component intensity ratio does not agree with experiment at intermediate scattering angles. Also the spread of the signal along the radial axis is often too narrow in theory. At the edge cases of small and large angles theory predicts the presence of a single peak which turned out to be 
correct. The strengths of the PESs are in their ability to model accurate energy losses and main scattering directions. They can also demonstrate the negligible influence of NQEs on the shown spectra. Of course the model system used in the simulations is not a one-to-one copy of the experiment. The larger Moiré structures and deviations from a perfectly flat graphene surface do contribute to the results.

\subsection{Conclusion and Outlook}

In this section was shown how an $\mathrm{H}$ atom interacts with a graphene surface on the (sub-) atomic scale. This was accomplished by reparametrizing the REBO PES based on two reference data sets generated on different levels of DFT. An optimization of the vanilla REBO potential was necessary because the discovery of graphene happened after publication of the PES. Therefore, graphene structures with their unique bond order characteristics could not have been taken into account in the original parametrization. Although in the process of fitting the PES loses its transferability to other hydrocarbons, it becomes accurate with respect to the system of interest in this work. Both optimized versions namely REBO-PBE and REBOEMFT are able to simulate the energy loss mechanism of an impinging $\mathrm{H}$ atom. It was also shown that this mechanism can be derived via the calculation of a few $a b$ initio MD trajectories on the level of DFT GGA. This approach is of course not feasible to obtain precise statistics on measures like energy loss distributions with angular resolution or polar incidence angle dependent sticking probabilities. It is for this reason that reparametrization is an essential part of this work especially to make meaningful comparisons with experiment and for the inclusion of NQEs.

It turns out, the effect of NQEs on energy loss distributions is small. They also do not affect the sticking probability as long as the barrier to adsorption is overcome not more than once. If barrier recrossing becomes important, the effect of $\mathrm{ZPE}$ in the $\mathrm{C}-\mathrm{H}$ bond reduces the sticking probability of both isotopes. Isotope substitution itself does influence sticking regardless of incidence energy. There exists an inertial kinetic isotope effect due to the carbon network having more time to re-hybridize during the longer lasting collision that leads to more energy transfer and more sticking if $\mathrm{D}$ atoms are used as projectiles. The addition of a $\operatorname{Pt}(111)$ substrate mostly enhances barrier crossing during the adsorption process. Sticking is enhanced at incidence energies up to $1 \mathrm{eV}$ due a reduced barrier and more carbon atom motion in the adsorption coordinate. In comparison with experiment the substrate decreases the intensity of the fast channel at the highest normal incidence 
energy in the angle-resolved energy loss plots. None of these methods captures the experimental findings accurately. In experiment there is a smooth transition from only the quasi-elastic peak at high polar angles to a coexistence region of both channels at intermediate values. At small polar angles only the slow channel is visible. Theory does reproduce this behavior, but the angle-outcome relationship deviates several degrees. In REBO-EMFT regardless whether NQEs or the substrate is simulated there is always the fast component present. The REBO-PBE PES correctly describes its disappearance towards small polar incidence angles. In return, this PES fails to predict the fast component at $\theta_{\mathrm{i}}=45^{\circ}$ which REBO-EMFT correctly captures.

In future projects one might try to fit parts of the REBO potential separately. In EMT there is one set of parameters that describes the projectile and one for the metal. The interaction is calculated from these atom-specific sets by mixing rules. In $\mathrm{REBO}$ though the $\mathrm{C}-\mathrm{C}$ and $\mathrm{C}-\mathrm{H}$ interaction is modeled via different parameter sets. Hence, one way to approach the parametrization would be to first fit the $\mathrm{C}-\mathrm{C}$ potential to accurately describe the graphene sheet by checking for quantities like the phonon spectrum or the bond stretch energy curve for $\mathrm{C}$ coming out of the surface plane. In a subsequent step $\mathrm{H}$ could be added to the training data thereby reducing parameter space subject to optimization because only the $\mathrm{C}-\mathrm{H}$ interaction parameters require updates. As far as parameter optimization itself is concerned it would be helpful to have analytic gradients available. Unfortunately there are no derivatives with respect to parameters available in the literature so one would need to calculate them by hand. This is of course an extended project by itself given the sophisticated REBO bond order equations, but it would lead to a speedup of approximately two orders of magnitude compared to the current numerical implementation.

Besides the actual parameters there are numerous uniform 2D and 3D knot vectors in the original REBO publication which define spline interpolation functions. They are crucial for continuous bond order changes for example when the particle approaches a $\mathrm{C}$ atom. They have been left unaltered in the current parametrizations as there are simply too many of them. But, one could think about identifying the relevant ones for this system and also include them in the optimization procedure. Another improvement would be to extend the energy range for which the parametrization yields valid results. The presented potentials have been thoroughly tested up to roughly $2 \mathrm{eV}$ projectile incidence energy, but not much beyond room temperature as far as the surface is concerned. But since thermal energies are rather small, I ran 
scattering simulations for testing purposes at $500 \mathrm{~K}$ which also seemed reasonable. Higher incidence energies should be used with caution though. A problem that might arise when training a PES up to $E_{\mathrm{i}}=10 \mathrm{eV}$ for example to model recent advances in this group with vacuum UV lasers is the loss of accuracy at small incidence energies. On the other hand, it would be really interesting to see $\mathrm{H}$ atoms penetrating the graphene sheet through the center of a carbon ring and to see them interact with the metal substrate. Maybe it is possible at a certain angle to first bring the $\mathrm{H}$ atom in contact with the substrate and make it recoil through the graphene surface after a single bounce off of the metal.

PESs based on Neural Networks could also be helpful with all kinds of $\mathrm{H}$ on graphene simulations [262]. First they are not constrained to any physically motivated mathematical form which would solve the problem of simultaneously being accurate at low and high incidence energies. Second the substrate would not have to be artificially connected to the graphene sheet by a parametrized LJ interaction. It would also provide an opportunity to use a strongly interacting metal as a substrate. There is experimental data available in this group of $\mathrm{H}$ atom scattering experiments from graphene on nickel. But since Ni forms a real chemical bond to carbon there needs to be one PES that describes all atomic species in this system at once which is currently beyond our capabilities.

From the experimental side it would be desirable to also detect atoms that scattered out-of-plane. Since theory predicts such a broad feature in the scattering signal as soon as transient $\mathrm{C}-\mathrm{H}$ bond formation occurs it would be a stringent test of theory. Moreover, it could be checked how well the fast component obeys cylindrical symmetry and the signal could directly be integrated without the need for approximations. In actual numbers less than $0.8 \%$ of the hemisphere above the graphene surface is accessible to the detector in experiment. From this point of view it is remarkable that so much data and ultimately insight could be extracted with this setup. Finally it would improve the comparison between theory and experiment because a large portion of simulated trajectories would not have to be disregarded due to out of plane scattering. 



\section{References}

[1] Kasemo, B.; Törnqvist, E.; Nørskov, J.; Lundqvist, B. Surf. Sci. 1979, 89, $554-565$.

[2] White, J. D.; Chen, J.; Matsiev, D.; Auerbach, D. J.; Wodtke, A. M. Nature 2005, 433, 503-505.

[3] Dorenkamp, Y.; Jiang, H.; Köckert, H.; Hertl, N.; Kammler, M.; Janke, S. M.; Kandratsenka, A.; Wodtke, A. M.; Bünermann, O. J. Chem. Phys. 2018, 148, 034706.

[4] Nienhaus, H. Surf. Sci. Rep. 2002, 45, 1-78.

[5] Kandratsenka, A.; Jiang, H.; Dorenkamp, Y.; Janke, S. M.; Kammler, M.; Wodtke, A. M.; Bünermann, O. Proc. Nat. Acad. Sci. 2018, 115, 680-684.

[6] Jiang, H.; Kammler, M.; Ding, F.; Dorenkamp, Y.; Manby, F. R.; Wodtke, A. M.; Miller, T. F.; Kandratsenka, A.; Bünermann, O. Science 2019, 364, 379-382.

[7] So, S. K.; Franchy, R.; Ho, W. J. Chem. Phys. 1989, 91, 5701-5706.

[8] Asada, H.; Matsui, T. Jpn. J. Appl. Phys. 1982, 21, 259-263.

[9] Asada, H. Surf. Sci. 1981, 110, 270-286.

[10] Kleyn, A. W.; Luntz, A. C.; Auerbach, D. J. Phys. Rev. Lett. 1981, 47, 11691172.

[11] McClelland, G. M.; Kubiak, G. D.; Rennagel, H. G.; Zare, R. N. Phys. Rev. Lett. 1981, 46, 831-834.

[12] Barker, J. A.; Kleyn, A. W.; Auerbach, D. J. Chem. Phys. Lett. 1983, 97, $9-13$.

[13] Rettner, C. T.; Fabre, F.; Kimman, J.; Auerbach, D. J. Phys. Rev. Lett. 1985, 55, 1904-1907.

[14] Rettner, C. T.; Kimman, J.; Fabre, F.; Auerbach, D. J.; Morawitz, H. Surf. Sci. 1987, 192, 107-130.

[15] Lucchese, R. R.; Tully, J. C. J. Chem. Phys. 1984, 80, 3451-3462. 
[16] Tully, J. C.; Gomez, M.; Martin, H.-G. J. Vac. Sci. Technol. A 1993, 11, $1914-1920$.

[17] Newns, D. M. Surf. Sci. 1986, 171, 600-614.

[18] Gross, A.; Brenig, W. Chem. Phys. 1993, 17r, 497-508.

[19] Gates, G. A.; Darling, G. R.; Holloway, S. J. Chem. Phys. 1994, 101, 62816288.

[20] Huang, Y.; Wodtke, A. M.; Hou, H.; Rettner, C. T.; Auerbach, D. J. Phys. Rev. Lett. 2000, 84, 4.

[21] Huang, Y.; Rettner, C. T.; Auerbach, D. J.; Wodtke, A. M. Science 2000, 290, 111-114.

[22] Krüger, B. C.; Meyer, S.; Kandratsenka, A.; Wodtke, A. M.; Schäfer, T. J. Phys. Chem. Lett. 2016, 7, 441-446.

[23] Díez Muiño, R., Ed. Dynamics of gas-surface interactions: atomic-level understanding of scattering processes at surfaces; Springer series in surface sciences 50; Springer: Berlin, 2013; OCLC: 840061450.

[24] Luntz, A. Chemical Bonding at Surfaces and Interfaces; Elsevier, 2008; pp $143-254$.

[25] Jensen, F. Introduction to Computational Chemistry, 2nd ed.; John Wiley \& Sons, Inc., 2006.

[26] Marx, D.; Hutter, J. Ab Initio Molecular Dynamics: Basic Theory and Advanced Methods; Cambridge University Press: Cambridge, 2009.

[27] Leszczynski, J., Ed. Handbook of computational chemistry; Springer reference; Springer: Dordrecht, 2012; OCLC: 838909280.

[28] Szabo, A.; Ostlund, N. S. Modern quantum chemistry: Introduction to advanced electronic structure theory; Dover Publications, 1996.

[29] Anderson, J. M. Mathematics for quantum chemistry; Courier Corporation, 2005.

[30] Hohenberg, P.; Kohn, W. Phys. Rev. 1964, 136, B864-B871. 
[31] Löwdin, P.-O. Int. J. Quantum Chem. 1985, 28, 19-37.

[32] Golden, S. Phys. Rev. 1957, 105, 604-615.

[33] Murphy, D. R. Phys. Rev. A 1981, 24, 1682-1688.

[34] Gáspár, R. Acta Physica 1954, 3, 263-286.

[35] Kohn, W.; Sham, L. J. Phys. Rev. 1965, 140, A1133-A1138.

[36] Wang, Y. A.; Govind, N.; Carter, E. A. Phys. Rev. B 1999, 60, 16350-16358.

[37] Perdew, J. P.; Chevary, J. A.; Vosko, S. H.; Jackson, K. A.; Pederson, M. R.; Singh, D. J.; Fiolhais, C. Phys. Rev. B 1992, 46, 6671-6687.

[38] Philipsen, P.; te Velde, G.; Baerends, E. Chem. Phys. Lett. 1994, 226, 583588.

[39] Perdew, J. P.; Burke, K.; Ernzerhof, M. Phys. Rev. Lett. 1996, 77, 3865-3868.

[40] Becke, A. D. J. Chem. Phys. 1993, 98, 1372-1377.

[41] Stroppa, A.; Picozzi, S. Phys. Chem. Chem. Phys. 2010, 12, 5405.

[42] Kim, K.; Jordan, K. D. J. Phys. Chem. 1994, 98, 10089-10094.

[43] Stephens, P. J.; Devlin, F. J.; Chabalowski, C. F.; Frisch, M. J. J. Phys. Chem. 1994, 98, 11623-11627.

[44] Fornace, M. E.; Lee, J.; Miyamoto, K.; Manby, F. R.; Miller, T. F. J. Chem. Theory Comput. 2015, 11, 568-580.

[45] Kurth, S.; Perdew, J. P.; Blaha, P. Int. J. Quantum Chem. 1999, 75, 889-909.

[46] Grimme, S.; Antony, J.; Ehrlich, S.; Krieg, H. J. Chem. Phys. 2010, 132, 154104.

[47] Tkatchenko, A.; Scheffler, M. Phys. Rev. Lett. 2009, 102.

[48] Tozer, D. J.; Handy, N. C. J. Chem. Phys. 1998, 109, 10180-10189.

[49] Cencek, W.; Szalewicz, K. J. Chem. Phys. 2013, 139, 024104. 
[50] Gross, E. K. U.; Dobson, J. F.; Petersilka, M. In Density Functional Theory II: Relativistic and Time Dependent Extensions; Nalewajski, R. F., Ed.; Topics in Current Chemistry; Springer Berlin Heidelberg: Berlin, Heidelberg, 1996; pp 81-172.

[51] Eriksen, J. J.; Sauer, S. P.; Mikkelsen, K. V.; Christiansen, O.; Jensen, H. J. A.; Kongsted, J. Mol. Phys. 2013, 111, 1235-1248.

[52] Neumann, R.; Nobes, R. H.; Handy, N. C. Mol. Phys. 1996, 87, 1-36.

[53] Paldus, J. In Theory and Applications of Computational Chemistry; Dykstra, C. E., Frenking, G., Kim, K. S., Scuseria, G. E., Eds.; Elsevier: Amsterdam, 2005; pp 115-147.

[54] Jones, J. E. Proc. R. Soc. A 1924, 106, 463-477.

[55] Goharshadi, E. K.; Abbaspour, M. J. Chem. Theory Comput. 2006, 2, 920926.

[56] Jacobsen, K. W.; Nørskov, J. K.; Puska, M. J. Phys. Rev. B 1987, 35, 74237442 .

[57] Jacobsen, K.; Stoltze, P.; Nørskov, J. Surf. Sci. 1996, 366, 394-402.

[58] Janke, S. M.; Auerbach, D. J.; Wodtke, A. M.; Kandratsenka, A. J. Chem. Phys. 2015, 143, 124708.

[59] Wadell, H. J. Geol. 1935, 43, 250-280.

[60] Wazzan, A. R.; Bristoti, A.; Robinson, L. B.; Ahmedieh, A. J. Appl. Phys. 1973, 44, 2018-2024.

[61] Murnaghan, F. D. Proc. Natl. Acad. Sci. 1944, 30, 244-247.

[62] Brenner, D. W. Phys. Rev. B 1990, 42, 9458-9471.

[63] Abell, G. C. Phys. Rev. B 1985, 31, 6184-6196.

[64] Rose, J. H.; Smith, J. R.; Ferrante, J. Phys. Rev. B 1983, 28, 1835-1845.

[65] Tersoff, J. Phys. Rev. B 1988, 37, 6991-7000.

[66] Tersoff, J. Phys. Rev. B 1989, 39, 5566-5568. 
[67] Ito, T.; Khor, K. E.; Das Sarma, S. Phys. Rev. B 1990, 41, 3893-3896.

[68] Brenner, D. W. Phys. Status Solidi B 2000, 217, 23-40.

[69] Brenner, D. W.; Shenderova, O. A.; Harrison, J. A.; Stuart, S. J.; Ni, B.; Sinnott, S. B. J. Phys. Condens. Matter 2002, 14, 783-802.

[70] Brenner, D. W.; Shenderova, O. A.; Areshkin, D. A. In Reviews in Computational Chemistry; Lipkowitz, K., Boyd, D., Eds.; Wiley-Blackwell, 2007; Vol. 12; pp 207-239.

[71] Slater, J. C.; Koster, G. F. Phys. Rev. 1954, 94, 1498-1524.

[72] Harris, J. Phys. Rev. B 1985, 31, 1770-1779.

[73] Lyapunov, A. M. General Problem of the Stability Of Motion; Taylor \& Francis: London, 1992; Russian original published by the Kharkov Mathematical Society (1892).

[74] Deiters, U. K. Z. Phys. Chem. 2013, 227, 345-352.

[75] Verlet, L. Phys. Rev. 1967, 159, 98-103.

[76] Juaristi, J. I.; Alducin, M.; Muiño, R. D.; Busnengo, H. F.; Salin, A. Phys. Rev. Lett. 2008, 100.

[77] Spiering, P.; Meyer, J. J. Phys. Chem. Lett. 2018, 9, 1803-1808.

[78] Rittmeyer, S. P.; Meyer, J.; Juaristi, J. I.; Reuter, K. Phys. Rev. Lett. 2015, 115 .

[79] Puska, M. J.; Nieminen, R. M. Phys. Rev. B 1983, 27, 6121-6128.

[80] Janke, S. Theoretical Description of Hydrogen Atom Scattering off Noble Metals. Dissertation, Georg August Universität, Göttingen, 2016.

[81] Caldin, E. F. Chem. Rev. 1969, 69, 135-156.

[82] Craig, I. R.; Manolopoulos, D. E. J. Chem. Phys. 2004, 121, 3368-3373.

[83] Habershon, S.; Manolopoulos, D. E.; Markland, T. E.; Miller, T. F. Ann. Rev. Phys. Chem. 2013, 64, 387-413. 
[84] Wipf, A. Statistical approach to quantum field theory: an introduction; Lecture notes in physics 864; Springer: Heidelberg, 2013; OCLC: 806019878.

[85] Miller, T. F.; Manolopoulos, D. E. J. Chem. Phys. 2005, 122, 184503.

[86] Ceriotti, M.; Parrinello, M.; Markland, T. E.; Manolopoulos, D. E. J. Chem. Phys. 2010, 133, 124104.

[87] Herman, M. F.; Bruskin, E. J.; Berne, B. J. J. Chem. Phys. 1982, 76, 51505155 .

[88] Markland, T. E.; Morrone, J. A.; Miyazaki, K.; Berne, B. J.; Reichman, D. R.; Rabani, E. J. Chem. Phys. 2012, 136, 074511.

[89] Welsch, R.; Song, K.; Shi, Q.; Althorpe, S. C.; Miller, T. F. J. Chem. Phys. 2016, 145, 204118.

[90] Lide, D. R., Ed. CRC Handbook of Chemistry and Physics, Internet Version; CRC Press: Boca Raton, FL, 2005; http://hbcponline.com.

[91] Andersen, H. C. J. Chem. Phys. 1980, 72, 2384-2393.

[92] Levenberg, K. Quart. Appl. Math. 1944, 2, 164-168.

[93] Marquardt, D. J. Soc. Indust. Appl. Math. 1963, 11, 164-168.

[94] Di, S.; Sun, W. Optim. Methods Softw. 1996, 6, 237-263.

[95] Intel@ Math Kernel Library - MKL 2019; Developer reference Revision 091, 2018; https://software.intel.com/sites/default/files/mkl-2019-developerreference-f.pdf.

[96] Yuan, Y.-X. Math. Program. 2015, 151, 249-281.

[97] Berghen, F. CONDOR: a constrained, non-linear, derivative-free parallel optimizer for continuous, high computing load, noisy objective functions. Dissertation, Université Libre de Bruxelles, Brussels, 2004; http://www.appliedmathematics.net/optimization/thesis_optimization.pdf.

[98] Darwin, C. On the origin of species by means of natural selection, or the preservation of favoured races in the struggle for life, 1st ed.; John Murray: London, 1859. 
[99] Goldberg, D. E. Genetic Algorithms in Search, Optimization, and Machine Learning, 1st ed.; Addison-Wesley: Boston, MA, USA, 1989.

[100] Dieterich, J.; Hartke, B. Mol. Phys. 2010, 108, 279-291.

[101] Ting, C. K. Adv. Artif. Life; Springer-Verlag, 2005.

[102] Sullivan, A. D.; Wigginton, J.; Kirschner, D. Proc. Nat. Acad. Sci. 2001, 98, 10214-10219.

[103] Holland, J. Adaptation in natural and artificial systems; MIT Press Cambridge: MA, USA, 1992.

[104] Taherdangkoo, M.; Paziresh, M.; Yazdi, M.; Bagheri, M. Open Eng. 2013, 3.

[105] Wolpert, D.; Macready, W. IEEE Trans. Evol. Comput. 1997, 1, 67-82.

[106] van Leeuwen, J. Handbook of Theoretical Computer Science; MIT Press; The MIT Press: Cambridge, MA, USA, 1994; Vol. A: Algorithms and Complexity.

[107] Wille, L. T.; Vennik, J. J. Phys. A 1985, 18, L419-L422.

[108] Kozlov, S. M.; Viñes, F.; Görling, A. J. Phys. Chem. C 2012, 116, 7360-7366.

[109] Bitzek, E.; Koskinen, P.; Gähler, F.; Moseler, M.; Gumbsch, P. Phys. Rev. Lett. 2006, 97.

[110] Kresse, G.; Hafner, J. Phys. Rev. B 1993, 47, 558-561.

[111] Kresse, G.; Hafner, J. Phys. Rev. B 1994, 49, 14251-14269.

[112] Kresse, G.; Furthmüller, J. Comput. Mater. Sci. 1996, 6, 15-50.

[113] Kresse, G.; Furthmüller, J. Phys. Rev. B 1996, 54, 11169-11186.

[114] Kresse, G.; Joubert, D. Phys. Rev. B 1999, 59, 1758-1775.

[115] Bloch, F. Z. Phys. 1929, 52, 555-600.

[116] Blöchl, P. E. Phys. Rev. B 1994, 50, 17953-17979.

[117] Kresse, G.; Marsman, M.; Furthmüller, J. VASP the GUIDE; manual, 2016; http://cms.mpi.univie.ac.at/vasp/vasp.pdf. 
[118] Kammler, M.; Janke, S. M.; Kandratsenka, A.; Wodtke, A. M. Chem. Phys. Lett. 2017, 683, 286-290.

[119] OpenMP Architecture Review Board, OpenMP Application Program Interface, Version 2.0.2; 2017; http://openmp.org.

[120] Cao, J.; Voth, G. A. J. Chem. Phys. 1994, 100, 5106-5117.

[121] Jang, S.; Voth, G. A. J. Chem. Phys. 1999, 111, 2371-2384.

[122] Hele, T. J. H.; Willatt, M. J.; Muolo, A.; Althorpe, S. C. J. Chem. Phys. 2015, 142, 134103.

[123] Hele, T. J. H.; Willatt, M. J.; Muolo, A.; Althorpe, S. C. J. Chem. Phys. 2015, 142, 191101.

[124] Willatt, M. J.; Ceriotti, M.; Althorpe, S. C. J. Chem. Phys. 2018, 148, 102336.

[125] de Jong, A.; Niemantsverdriet, J. Surf. Sci. 1990, 233, 355-365.

[126] Binnig, G.; Rohrer, H. Surf. Sci. 1983, 126, 236-244.

[127] Davisson, C.; Germer, L. H. Nature 1927, 119, 558.

[128] Thomson, G. P.; Reid, A. Nature 1927, 119, 890.

[129] Economou, E. N. Phys. Rev. 1969, 182, 539-554.

[130] Lucas, A. A.; Šunjić, M. Phys. Rev. Lett. 1971, 26, 229-232.

[131] London, F. Ztschr. Elektrochem. 1929, 35, 552-555.

[132] McCreery, J. H.; Wolken, G. J. Chem. Phys. 1975, 63, 2340-2349.

[133] McCreery, J. H.; Wolken, G. J. Chem. Phys. 1975, 63, 4072.

[134] Wolken, G. J. Chem. Phys. 1978, 68, 4338-4342.

[135] Avdeev, V. I.; Upton, T. H.; Weinberg, W. H.; Goddard, W. A. Surf. Sci. 1980, 95, 391-402.

[136] Sheng, J.; Zhang, J. Z. H. J. Chem. Phys. 1993, 99, 1373-1381.

[137] Kara, A.; DePristo, A. E. J. Chem. Phys. 1988, 88, 2033-2035. 
[138] Khanra, B. C.; Saha, S. K. Chem. Phys. Lett. 1983, 95, 4.

[139] Martin-Gondre, L.; Crespos, C.; Larregaray, P.; Rayez, J.; van Ootegem, B.; Conte, D. Chem. Phys. Lett. 2009, 471, 136-142.

[140] Martin-Gondre, L.; Crespos, C.; Larregaray, P.; Rayez, J. C.; van Ootegem, B.; Conte, D. J. Chem. Phys. 2010, 132, 204501.

[141] Morón, V.; Martin-Gondre, L.; Crespos, C.; Larregaray, P.; Gamallo, P.; Sayós, R. Comput. Theor. Chem. 2012, 990, 132-143.

[142] Morón, V.; Martin-Gondre, L.; Gamallo, P.; Sayós, R. J. Phys. Chem. C 2012, $116,21482-21488$.

[143] Daw, M. S.; Baskes, M. I. Phys. Rev. Lett. 1983, 50, 1285-1288.

[144] Daw, M. S.; Baskes, M. I. Phys. Rev. B 1984, 29, 6443-6453.

[145] Jianjun, X.; Ping, J.; Kaiming, Z. J. Phys. Condens. Matter 1994, 6, 1219 1228.

[146] Jianjun, X.; Ping, J.; Kaiming, Z. J. Phys. Condens. Matter 1994, 6, 72177226.

[147] Xie, J.; Jiang, P.; Zhang, K. J. Chem. Phys. 1996, 104, 9994-10000.

[148] Sun, Q.; Xie, J.; Zhang, T. Surf. Sci. 1995, 338, 11-18.

[149] Koido, T.; Ito, D.; Tokumasu, T.; Tomarikawa, K.; Yonemura, S. ECS Trans. 2010, 25, 59-68.

[150] Norskov, J. K. Rep. Prog. Phys. 1990, 53, 1253-1295.

[151] Wang, Y.; Perdew, J. P. Phys. Rev. B 1991, 44, 13298-13307.

[152] Zhang, Y.; Yang, W. Phys. Rev. Lett. 1998, 80, 890-890.

[153] Monkhorst, H. J.; Pack, J. D. Phys. Rev. B 1976, 13, 5188-5192.

[154] Methfessel, M.; Paxton, A. T. Phys. Rev. B 1989, 40, 3616-3621.

[155] Perdew, J. P.; Ruzsinszky, A.; Csonka, G. I.; Vydrov, O. A.; Scuseria, G. E.; Constantin, L. A.; Zhou, X.; Burke, K. Phys. Rev. Lett. 2008, 100. 
[156] Siethoff, H. Phys. Status Solidi 1997, 200, 57-66.

[157] Wan, J.; Fan, Y. L.; Gong, D. W.; Shen, S. G.; Fan, X. Q. Model. Simul. Mater. Sci. Eng. 1999, \%, 189-206.

[158] Janke, S. M.; Pavanello, M.; Kroes, G.-J.; Auerbach, D.; Wodtke, A. M.; Kandratsenka, A. Z. Phys. Chem. 2013, 227.

[159] Nobuhara, K.; Nakanishi, H.; Kasai, H.; Okiji, A. J. Appl. Phys. 2000, 88, 6897-6901.

[160] Groß, A. Theoretical surface science: a microscopic perspective, 2nd ed.; Springer: Berlin, 2009; OCLC: 463781287.

[161] Simmons, G.; Wang, H. Single crystal elastic constants and calculated aggregate properties: a handbook; M.I.T. Press, 1971.

[162] Hoss, A.; Nold, M.; von Blanckenhagen, P.; Meyer, O. Phys. Rev. B 1992, 45, $8714-8720$.

[163] Hoss, A.; Romahn, U.; Nold, M. Europhys. Lett. 1992, 20, 125-130.

[164] Marzari, N.; Vanderbilt, D.; De Vita, A.; Payne, M. C. Phys. Rev. Lett. 1999, 82, 3296-3299.

[165] Barnett, R. N.; Landman, U. Phys. Rev. B 1991, 44, 3226-3239.

[166] Rahman, T. S.; Tian, Z.; Black, J. E. Surf. Sci. 1997, 374, 9-16.

[167] Wada, Y.; Yanagishita, T.; Masuda, H. Anal. Chem. 2007, 79, 9122-9127.

[168] Bünermann, O.; Jiang, H.; Dorenkamp, Y.; Kandratsenka, A.; Janke, S. M.; Auerbach, D. J.; Wodtke, A. M. Science 2015, 350, 1346-1349.

[169] Baule, B. Ann. Phys. 1914, 349, 145-176.

[170] LaRue, J.; Schäfer, T.; Matsiev, D.; Velarde, L.; Nahler, N. H.; Auerbach, D. J.; Wodtke, A. M. Phys. Chem. Chem. Phys. 2011, 13, 97-99.

[171] Trail, J. R.; Graham, M. C.; Bird, D. M.; Persson, M.; Holloway, S. Phys. Rev. Lett. 2002, 88 .

[172] Trail, J. R.; Bird, D. M.; Persson, M.; Holloway, S. J. Chem. Phys. 2003, 119, 4539-4549. 
[173] Lindenblatt, M.; van Heys, J.; Pehlke, E. Surf. Sci. 2006, 600, 3624-3628.

[174] Lindenblatt, M.; Pehlke, E.; Duvenbeck, A.; Rethfeld, B.; Wucher, A. Nucl. Instrum. Methods Phys. Res. 2006, 246, 333-339.

[175] Monturet, S.; Saalfrank, P. Phys. Rev. B 2010, 82.

[176] Schindler, B.; Diesing, D.; Hasselbrink, E. J. Chem. Phys. 2011, 134, 034705.

[177] Mildner, B.; Hasselbrink, E.; Diesing, D. Chem. Phys. Lett. 2006, 432, 133138.

[178] Krix, D.; Nünthel, R.; Nienhaus, H. Phys. Rev. B 2007, 75.

[179] Nienhaus, H.; Bergh, H. S.; Gergen, B.; Majumdar, A.; Weinberg, W. H.; McFarland, E. W. Phys. Rev. Lett. 1999, 82, 446-449.

[180] Nagasaka, M.; Kosugi, N.; Rühl, E. J. Chem. Phys. 2012, 136, 234312.

[181] Sonnenblick, Y.; Kalman, Z.; Steinberger, I. J. Cryst. Growth 1982, 58, 143151.

[182] Oh, S.-K. J. Thermodyn. 2013, 2013, 29.

[183] Tsongas, G. A.; Koutsoyannis, S. P. J. Phys. B 1969, 2, 437-441.

[184] Rubbo, M.; Bruno, M. Colloid Journal 2016, 78, 658-668.

[185] Kim, S. U.; Monroe, C. W. J. Comput. Phys. 2014, 273, 358-373.

[186] Krainyukova, N. V. Thin Solid Films 2006, 515, 1658-1663.

[187] Hart, J. R.; Rappé, A. K. J. Chem. Phys. 1992, 97, 1109-1115.

[188] Grimme, S. J. Comput. Chem. 2006, 27, 1787-1799.

[189] Jasper, A. W.; Miller, J. A. Combust. Flame 2014, 161, 101-110.

[190] Lorentz, H. A. Ann. Phys. 1881, 248, 127-136.

[191] Hertl, N. personal communication. 2017; Georg August University, Göttingen.

[192] Krüger, K. personal communication. 2018; Georg August University, Göttingen. 
[193] Askerka, M.; Maurer, R. J.; Batista, V. S.; Tully, J. C. Phys. Rev. Lett. 2016, 116.

[194] Coffman, A. J.; Subotnik, J. E. Phys. Chem. Chem. Phys. 2018, 20, 98479854.

[195] Dou, W.; Subotnik, J. E. J. Chem. Phys. 2016, $144,024116$.

[196] Kroto, H. W.; Heath, J. R.; O'Brien, S. C.; Curl, R. F.; Smalley, R. E. Nature 1985, 318, 162 .

[197] Iijima, S. Nature 1991, 354, 56.

[198] Novoselov, K. S. Science 2004, 306, 666-669.

[199] Qin, Z.; Jung, G. S.; Kang, M. J.; Buehler, M. J. Sci. Adv. 2017, 3, e1601536.

[200] Akturk, A.; Goldsman, N. J. Appl. Phys. 2008, 103, 053702.

[201] Chen, J.-H.; Jang, C.; Xiao, S.; Ishigami, M.; Fuhrer, M. S. Nat. Nanotechnol. 2008, 3, 206-209.

[202] Balandin, A. A.; Ghosh, S.; Bao, W.; Calizo, I.; Teweldebrhan, D.; Miao, F.; Lau, C. N. Nano Lett. 2008, 8, 902-907.

[203] Changgu, L.; Wei, X.; Kysar, J. W.; Hone, J. Science 2008, 321, 385-388.

[204] Novoselov, K. S.; Jiang, D.; Schedin, F.; Booth, T. J.; Khotkevich, V. V.; Morozov, S. V.; Geim, A. K. Proc. Natl. Acad. Sci. 2005, 102, 10451-10453.

[205] Morisset, S.; Ferro, Y.; Allouche, A. J. Chem. Phys. 2010, 133, 044508.

[206] Houtte, D. v.; Martin, G.; Bécoulet, A.; Bucalossi, J.; Giruzzi, G.; Hoang, G. T.; Loarer, T.; Saoutic, B. Nucl. Fusion 2004, 44, L11-L15.

[207] Pégourié, B.; Brosset, C.; Delchambre, E.; Loarer, T.; Roubin, P.; Tsitrone, E.; Bucalossi, J.; Gunn, J.; Khodja, H.; Lafon, C.; Martin, C.; Parent, P.; Reichle, R. Phys. Scr. 2004, 2004, 23.

[208] Counsell, G. et al. Plasma Phys. Control. Fusion 2006, 48, B189-B199.

[209] Meregalli, V.; Parrinello, M. Appl. Phys. A 2001, 72, 143-146. 
[210] Nagar, R.; Vinayan, B. P.; Samantaray, S. S.; Ramaprabhu, S. J. Mater. Chem. A 2017, 5, 22897-22912.

[211] Tozzini, V.; Pellegrini, V. Phys. Chem. Chem. Phys. 2013, 15, 80-89.

[212] Wu, C.-D.; Fang, T.-H.; Lo, J.-Y.; Feng, Y.-L. J. Mol. Model. 2013, 19, 38133819.

[213] Camiola, V. D.; Farchioni, R.; Pellegrini, V.; Tozzini, V. 2D Mater. 2015, 2, 014009.

[214] de Tomas, C.; Suarez-Martinez, I.; Marks, N. A. Carbon 2016, 109, 681-693.

[215] Sun, H. J. Phys. Chem. B 1998, 102, 7338-7364.

[216] Rappe, A. K.; Casewit, C. J.; Colwell, K. S.; Goddard, W. A.; Skiff, W. M. J. Am. Chem. Soc. 1992, 114, 10024-10035.

[217] van Duin, A. C. T.; Dasgupta, S.; Lorant, F.; Goddard, W. A. J. Phys. Chem. A 2001, 105, 9396-9409.

[218] Nouranian, S.; Gwaltney, S. R.; Baskes, M. I.; Tschopp, M. A.; Horstemeyer, M. F. Chem. Phys. Lett. 2015, 635, 278-284.

[219] Nouranian, S.; Tschopp, M. A.; Gwaltney, S. R.; Baskes, M. I.; Horstemeyer, M. F. Phys. Chem. Chem. Phys. 2014, 16, 6233-6249.

[220] Stuart, S. J.; Tutein, A. B.; Harrison, J. A. J. Chem. Phys. 2000, 112, 64726486.

[221] Plimpton, S. J.; Thompson, A. P. MRS Bulletin 2012, 37, 513-521.

[222] Ghio, E.; Mattera, L.; Salvo, C.; Tommasini, F.; Valbusa, U. J. Chem. Phys. 1980, 73, 556-561.

[223] Bonfanti, M.; Martinazzo, R.; Tantardini, G. F.; Ponti, A. J. Phys. Chem. C 2007, 111, 5825-5829.

[224] Blöchl, P. E.; Jepsen, O.; Andersen, O. K. Phys. Rev. B 1994, 49, 1622316233.

[225] Wang, Y.; Qian, H.-J.; Morokuma, K.; Irle, S. J. Phys. Chem. A 2012, 116, 7154-7160. 
[226] Froese, R. D. J.; Humbel, S.; Svensson, M.; Morokuma, K. J. Phys. Chem. A 1997, 101, 227-233.

[227] Watts, J. D.; Gauss, J.; Bartlett, R. J. J. Chem. Phys. 1993, 98, 8718-8733.

[228] Dunning, T. H. J. Chem. Phys. 1989, 90, 1007-1023.

[229] Sha, X.; Jackson, B. Surf. Sci. 2002, 496, 318-330.

[230] Becke, A. D. J. Chem. Phys. 1993, 98, 5648-5652.

[231] Ding, F.; Manby, F. R.; Miller, T. F. J. Chem. Theory Comput. 2017, 13, $1605-1615$.

[232] Ding, F.; Tsuchiya, T.; Manby, F. R.; Miller, T. F. J. Chem. Theory Comput. 2017, 13, 4216-4227.

[233] Vosko, S. H.; Wilk, L.; Nusair, M. Can. J. Phys. 1980, 58, 1200-1211.

[234] Hehre, W. J.; Stewart, R. F.; Pople, J. A. J. Chem. Phys. 1969, 51, 2657-2664.

[235] Manby, F. et al. 2019,

[236] Lendvay, G. J. Mol. Struct. Theochem 2000, 501-502, 389-393.

[237] Miller III, T. F.; Habershon, S.; Markland, T. E.; Richardson, J. O. personal communications. 2018.

[238] Kaufmann, S.; Schwarzer, D.; Reichard, C.; Wodtke, A. M.; Bünermann, O. Nat. Commun. 2014, 5.

[239] Jiang, H. Dynamics of Hydrogen Atoms Scattering from Surfaces. Dissertation, Georg August Universität, Göttingen, 2016.

[240] Herrero, C. P.; Ramírez, R. Phys. Rev. B 2009, 79.

[241] Brihuega, I.; Yndurain, F. J. Phys. Chem. B 2018, 122, 595-600.

[242] Kim, H.; Balgar, T.; Hasselbrink, E. Chem. Phys. Lett. 2012, 546, 12-17.

[243] Paris, A. et al. Adv. Funct. Mater. 2013, 23, 1628-1635.

[244] Richardson, J. O. J. Chem. Phys. 2018, 148, 200901.

[245] Petersen, J.; Pollak, E. J. Phys. Chem. A 2018, 122, 3563-3571. 
[246] Sutter, P.; Sadowski, J. T.; Sutter, E. Phys. Rev. B 2009, 80.

[247] Andersen, M.; Hornekær, L.; Hammer, B. Phys. Rev. B 2012, 86.

[248] Khomyakov, P. A.; Giovannetti, G.; Rusu, P. C.; Brocks, G.; van den Brink, J.; Kelly, P. J. Phys. Rev. B 2009, 79.

[249] Aizawa, T.; Hwang, Y.; Hayami, W.; Souda, R.; Otani, S.; Ishizawa, Y. Surf. Sci. 1992, 260, 311-318.

[250] Al Taleb, A.; Farías, D. J. Phys. Condens. Matter 2016, 28, 103005.

[251] Politano, A.; Marino, A. R.; Campi, D.; Farías, D.; Miranda, R.; Chiarello, G. Carbon 2012, 50, 4903-4910.

[252] Hamada, I.; Otani, M. Phys. Rev. B 2010, 82.

[253] Cooper, V. R. Phys. Rev. B 2010, 81.

[254] Lee, K.; Murray, E. D.; Kong, L.; Lundqvist, B. I.; Langreth, D. C. Phys. Rev. $B$ 2010, 82.

[255] Toyoda, K.; Nozawa, K.; Matsukawa, N.; Yoshii, S. J. Phys. Chem. C 2013, $117,8156-8160$.

[256] Giovannetti, G.; Khomyakov, P. A.; Brocks, G.; Karpan, V. M.; van den Brink, J.; Kelly, P. J. Phys. Rev. Lett. 2008, 101.

[257] Gao, M.; Pan, Y.; Huang, L.; Hu, H.; Zhang, L. Z.; Guo, H. M.; Du, S. X.; Gao, H.-J. Appl. Phys. Lett. 2011, 98, 033101.

[258] Zecho, T.; Güttler, A.; Sha, X.; Jackson, B.; Küppers, J. J. Chem. Phys. 2002, $117,8486-8492$.

[259] Kerwin, J.; Jackson, B. J. Chem. Phys. 2008, 128, 084702.

[260] Aizawa, T.; Souda, R.; Otani, S.; Ishizawa, Y.; Oshima, C. Phys. Rev. B 1990, 42, 11469-11478.

[261] Hornekær, L.; Šljivančanin, v.; Xu, W.; Otero, R.; Rauls, E.; Stensgaard, I.; Laegsgaard, E.; Hammer, B.; Besenbacher, F. Phys. Rev. Lett. 2006, 96.

[262] Behler, J. Phys. Chem. Chem. Phys. 2011, 13, 17930. 


\section{Acronyms}

AIMD ab initio Molecular Dynamics.

bcc body-centered cubic.

BOA Born-Oppenheimer Approximation.

CMD Centroid Molecular Dynamics.

DFT Density Functional Theory.

DOF degree of freedom.

EAM Embedded Atom Method.

ehp electron-hole pair.

EMFT Embedded Mean-Field Theory.

EMT Effective Medium Theory.

fcc face-centered cubic.

FIRE Fast Inertial Relaxation Engine.

GA Genetic Algorithm.

GGA Generalized Gradient Approximation.

HF Hartree-Fock.

KIE kinetic isotope effect.

KS Kohn-Sham.

LDA Local Density Approximation.

LDFA Local Density Friction Approximation.

LEPS London-Eyring-Polanyi-Sato.

LJ Lennard-Jones. 
LM Levenberg-Marquardt.

MB Maxwell-Boltzmann.

MD Molecular Dynamics.

NQE Nuclear Quantum Effect.

PBE Perdew, Burke, and Ernzerhof.

PEC Potential Energy Curve.

PES Potential Energy Surface.

PILE Path Integral Langevin Equation.

REBO Reactive Empirical Bond Order.

RMSE Root Mean Square Error.

RP Ring Polymer.

RPMD Ring Polymer Molecular Dynamics.

SCF Self-Consistent Field.

TR Trust Region.

UEG Uniform Electron Gas.

VASP Vienna $a b$ initio Simulation Package.

ZPE Zero-Point Energy. 